\title{
OBSERVATIONS OF TUBULENCE, INTERNAL WAVES AND BACKGROUND FLOWS: AN INQUIRY INTO THE RELATIONSHIPS BETWEEN SCALES OF MOTION
}

by

\section{Kurt Louis Polzin}

B.A., Whitman College

(1986)

Submitted in partial fulfillment of the requirements for the degree of

Doctor of Philosophy

at the

MASSACHUSETTS INSTITUTE OF TECHNOLOGY

and the

WOODS HOLE OCEANOGRAPHIC INSTITUTION

September 1992

() Kurt Polzin 1992

The author hereby grants to MIT and WHOI pernyission to reproduce and distribute copies offthis thesis document in whole or in part.

Signature of Author

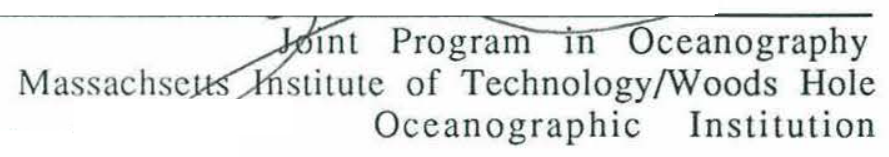

Certified by

John M. Toole

Thesis Supervisor

Accepted by

\begin{tabular}{|c|}
\hline MARINE \\
B!OLCGICAL \\
LABORATORY \\
\hline LIBRARY \\
\hline $\begin{array}{c}\text { WOOOS HOLE, MASSS. } \\
\text { W. H. O. I. }\end{array}$ \\
\hline
\end{tabular}

Lawrence J. Pratt Chairman, Joint Committee for Physical Oceanography, Massachusetts Institute of Technology/Woods Hole Oceanographic Institution 


\title{
OBSERVATIONS OF TURBULENCE, INTERNAL WAVES AND BACKGROUND FLOWS: AN INQUIRY INTO THE RELATIONSHIPS BETWEEN SCALES OF MOTION
}

\author{
by \\ Kurt Louis Polzin \\ Submitted in partial fulfillment of the requirements for the degree of \\ Doctor of Philosophy at the Massachusetts Institute of Technology \\ and the Woods Hole Oceanographic Institution \\ September 15, 1992
}

\begin{abstract}
Oceanic profiles of temperature, salinity, horizontal velocity, rate of dissipation of turbulent kinetic energy $(\varepsilon)$ and rate of dissipation of thermal variance $(\chi)$ are used to examine the parameterization of turbulent mixing in the ocean due to internal waves. Turbulent mixing is quantified through eddy diffusivity parameterizations of the mass $\left(\mathrm{K}_{\mathrm{p}}\right.$; Osborn, 1980) and heat fluxes $\left(\mathrm{K}_{\mathrm{T}}\right.$; Osborn and Cox, 1972) in turbulent production/dissipation balances. Turbulence in the ocean is generally held to result from the occurrence of shear instability in regions where the Richardson number is locally supercritical (i.e. $\mathrm{Ri} \leq 1 / 4$ ), permitting the growth of small-scale waves which break and result in turbulent mixing. The occurrence of shear instability results from the local intensification of the shear in the internal wave field. The energy dissipated in such events is provided by the energy flux to higher wavenumber due to nonlinear wave/wave interactions on scales of 10's to 100's of meters. In turn, the strength of the wave/wave interactions depends generally on the energy content of the internal wave field, which can vary considerably over even larger scales due to the presence of topography or background flows. The magnitude of turbulent mixing is linked to internal wave dynamics by equating the turbulent dissipation with the energy flux through the vertical wavenumber spectrum under the priviso that the model spectrum which forms the basis for the analysis is statistically stationary with respect to the nonlinear interactions. Dynamical models (McComas and Muller, 1981; Henyey et al., 1986) indicate that the Garrett and Munk (GM; Munk, 1981) spectrum is stationary. Observations from the far field of a seamount in a region of negligible large-scale flow were examined to address the issue of the buoyancy scaling of $\varepsilon$. These data exhibited large variations in background stratification with depth, but the internal wave characteristics were not substantially differentiable from the GM prescription. The magnitude of $\varepsilon$ and its functional dependence upon internal wave energy levels (E) and buoyancy frequency (N) was best described by the dynamical model of Henyey et al. (1986) $(\varepsilon \sim$ $\mathrm{E}^{2} \mathrm{~N}^{2}$ ). The Richardson number scaling model of Kunze et al. (1990) produced consistent estimates. A second dynamical model, McComas and Muller (1981), predicted an appropriate (E,N) scaling, but overestimated the
\end{abstract}


observed dissipation rates by a factor of five. Two kinematical dissipation parameterizations (Gargett and Holloway (1984) and Munk (1981)) predicted buoyancy scalings of $\mathrm{N}^{3 / 2}$ which were inconsistent with the observed scaling.

Data from an upper-ocean front, a warm core ring and a region of steep topography were analyzed in order to examine the parameter dependence of $\varepsilon$ in internal wave fields which exhibited potentially nonstationary characteristics. Evidence was provided which implied the internal wave field in an upper ocean front was interacting with and modified by the background flow. Inhomogeneity and anisotropy of the internal wave field were noted in that data set. The model of Gregg (1989), which in turn was based upon the model of Henyey et al., effectively collapsed the observed diffusivity estimates from the front. The warm core ring profiles were noted to be anisotropic, dominated by near-inertial frequencies and to have a peaked vertical wavenumber shear spectrum. The data from a region of steep topography were noted to have a peaked vertical wavenumber spectrum and were characterized by higher than GM frequency motions. For the latter two data sets, application of a frequency based correction to the Henyey et al. model (Henyey, 1991) reduced more than an order of magnitude scatter in the parameterized estimates of $\varepsilon$ to less than a factor of four. Of the possible non-equilibrium conditions in the internal wave field, the $(\mathrm{E}, \mathrm{N})$ scaled dissipation rates were most sensitive to deviations in wave field frequency content.

On the basis of a number of theoretical Richardson number probability distributions $\left(\mathrm{Ri}=\mathrm{N}^{2} / \mathrm{S}^{2}\right.$, where $\mathrm{S}^{2}$ is the sum of the squared vertical derivatives of horizontal velocity), the nominal dissipation scaling of the Kunze et al. model was determined to be $\mathrm{E}^{2} \mathrm{~N}^{3}$. This scaling is altered to the observed $\varepsilon \sim \mathrm{E}^{2} \mathrm{~N}^{2}$ scaling by a statistical dependence between $\mathrm{N}^{2}$ and $\mathrm{S}^{2}$ which reduces the occurrence of supercritical Ri values. This statistical dependence is hypothesized to be an effect of the turbulent momentum and buoyancy fluxes on the internal wave shear and strain profiles caused by shear instability. The statistical dependence between $\mathrm{N}^{2}$ and $\mathrm{S}^{2}$ exhibited a buoyancy scaling which was interpreted as resulting from the decreasing ratio between the time scale of the shear instability mechanism $[\mathrm{T} \sim 2 \pi / \mathrm{N}]$ and the adiabatic time scale $\left[\mathrm{T} \sim 2 \pi /(\mathrm{Nf})^{1 / 2}\right]$ of the internal wave field (f is the Coriolis parameter). This phenomenology is interpreted in light of saturated spectral theories which suggest that the magnitude and shape of the vertical wavenumber spectrum is controlled by instability mechanisms at large wavenumber $(\geq .1 \mathrm{cpm})$. We argue that saturated spectral theories are valid only in the limit where a separation exists between the two time scales, i.e. for large $\mathrm{N}$, low internal wave frequency content, and small $\mathrm{f}$.

These results have immediate implications for oceanic mixing driven by internal wave motions. First, background diffusivities are small: at GM energy levels, $K_{\rho} \sim .03 \times 10^{-4} \mathrm{~m}^{2} / \mathrm{s}\left(\mathrm{K}_{\rho}=.25 \varepsilon / \mathrm{N}^{2}\right)$. Secondly, since $\mathrm{K}_{\rho}$ is independent of $\mathrm{N}$ at constant $\mathrm{E}$, some process or collection of processes must be responsible for heightened $\mathrm{E}$ values in the abyss if internal waves cause the $\mathrm{O}\left(1-10 \times 10^{-4} \mathrm{~m}^{2} / \mathrm{s}\right)$ diffusivities generally inferred from deep ocean hydrographic data. We view internal wave reflection and/or internal wave generation associated with topographic features to be likely candidates.

Thesis Supervisor:

Dr. John M. Toole, Associate Scientist

Woods Hole Oceanographic Institution 


\section{Acknowledgments}

John Toole is to be thanked for his efforts as an advisor. His obvious confidence in my abilities was greatly appreciated, particularly in moments when those abilities were not quite so obvious to the author. John Toole and Ray Schmitt are to be thanked for giving me virtually unrestricted access to their data sets. Neil Oakey generously allowed the use of his data from the Endeavor 141 cruise. That data proved to be significant in interpreting and supporting the conclusions drawn from the High Resolution Profiler data. Moreover, Neil is to be thanked for his time and effort in initiating the author to the wonders of microstructure processing and for permitting the use of his processing algorithms. I have had many insightful conversations with Eric Kunze. I am particularly indebted to him for bringing the manuscript of Henyey (1991) to my attention and for suggesting the use of the shear/strain ratio to assess the frequency content of the internal wave field. Ellyn Montgomery assisted with the programming and data processing. Her efforts greatly reduced mine.

I have had the incredible fortune of being acquainted with Becky Schudlich, Joan Silberlicht and Deb Berglin. Their love and friendship have changed my world. 


\section{Contents}

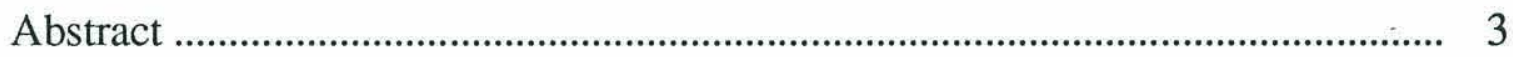

Acknowledgments .......................................................................................

I Introduction ..........................................................................

I I Fine- and Microstructure Characteristics Across the Northwest Atlantic Subtropical Front

2.1 Introduction .................................................................. 25

2.2 Finescale Sampling and Background Velocity Structure................................ $\quad 30$

2.3 Analysis Methods

2.3a Separation of Wave / Mean Flow ................ 37

2.3b Binning …............................................................. 39

2.3c Spectra ................................................................. 41

2.4 General Character of Finescale Velocity

2.4a CW vs CCW Signal in Velocity ....................... 42

2.4b WKB Scaling of Shear Variance .................... 45

2.5 Finescale Variability vs Frontal Position

2.5a Rotary Estimates ............................................... 48

2.5b Across-Front / Along-Front Anisotropy.... 48

2.5c Time Variability................................................. 54

2.6 Mixing Intensities ………………………................. 55

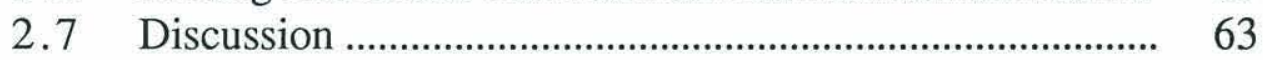

II I Finescale Parameterizations of Turbulent Dissipation

3.1 Introduction ................................................................ 73

3.2 A Review of Models of Dissipation Due to Internal Waves ................................................................ 77

3.3 The Criticism of Gregg (1989) by Gargett (1990) 88

3.4 Data Analysis ................................................................. 96

3.5 Model/Data Comparison

3.5a The N Dependence ............................................ 99

3.5b $(\mathrm{E}, \omega)$ Scaling …..................................................... 108

3.6 Discussion and Conclusions ……………................... 116 
IV A Richardson Number Model of Mixing and Statistics of Richardson Number

4.1 Introduction ................................................................. 125

4.2 Qualitative Evidence for the Shear Instability Mechanism .................................................................... 127

4.3 The Richardson Number Dissipation Parameterization of Kunze, Williams and Briscoe (1990) .......................................................... 138

4.4 Statistics of the Richardson Number ..................... 147

4.5 Dynamics of the Transition Region

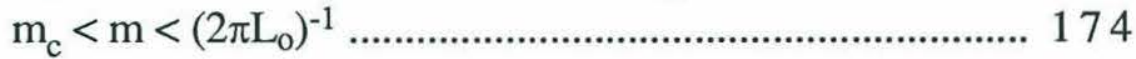

4.6 Conclusion and Discussion .......................................... 188

V Conclusion................................................................................... 191

Appendix One Fine- and Microstructure Processing

A.1 a Overview of the HRP .................................................... 203

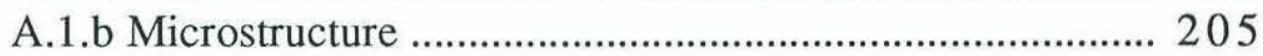

A.1.c Finescale Data ................................................................. 220

Appendix Two The Garrett and Munk Spectrum ............................ 225

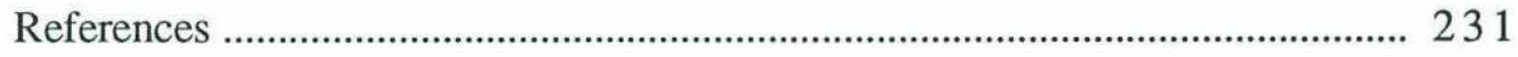


Chapter One

Introduction

The goal of this thesis is to provide the background with which to predict global patterns of turbulent mixing in the ocean due to internal waves. This work quantifies the relationships between the internal wave field, the occurrence of mixing events and the magnitude of the turbulent mixing. An internally consistent picture of mixing in the ocean is produced with the ability to parameterize internal wave related mixing both in terms of internal wave velocity and displacement over scales larger than 10 meters and in terms of mixing event statistics over smaller scales. These parameterizations provide the background with which to predict global patterns of mixing. Such global patterns are important to understanding oceanic dynamics and thermodynamics as the magnitude and spatial variability of mixing plays a role in determining the strength and structure of the thermohaline circulation. The quantitative prediction of global patterns of mixing and the determination of the effect upon the thermohaline circulation is beyond the scope of this thesis. However, this work does provide strong indications for the direction of further research.

What happens over one centimeter scales has broad implications for the dynamics and thermodynamics of the general circulation. Given that the deep density field is in steady state, the constant production of bottom water implies that vertical mixing processes are responsible for maintaining the observed stratification. 
Wyrtki (1961) employed a vertical advection/diffusion balance for the heat equation to demonstrate that the observed vertical distribution of potential temperature in the Pacific could be reconciled with the upwelling of bottom water balancing the vertical diffusion of heat. Given various estimates for the rate of production of bottom water and the assumption that the ratio between the average vertical velocity and the rate of turbulent diffusion was constant, an average vertical eddy diffusivity of $1 \times 10^{-4} \mathrm{~m}^{2} / \mathrm{s}$ was determined. This value is often referenced as a benchmark. Munk (1966) utilized a similar balance and then considered the various processes which could possibly lead to a turbulent mixing coefficient of that magnitude. While this coefficient is somewhat uncertain due to the use of the vertical advection/diffusion balance, the continuous production of dense water implies conversion via non-adiabatic processes elsewhere if the observed density field is to be maintained in a steady state.

Both the strength and structure of the thermohaline circulation are related to the stratification by the thermal wind relation. Smallscale processes are in turn related to the thermohaline circulation through their influence on the stratification rate. In a large scale numerical general circulation model (the GFDL model), the strength of the meridional overturning cell was found to depend on the magnitude of the vertical mixing as approximately the cube root of the eddy diffusivity (Bryan, 1987). A much larger dependence of the poleward heat transport upon the magnitude of the vertical eddy diffusivity was noted. A stratification dependent diffusivity, inversely proportional to the buoyancy frequency, was implemented 
in the GFDL general circulation model by Cummins et al. (1990) and the results compared to the case of constant diffusivity (Bryan, 1987). Cummins et al. found that the use of a stratification dependant diffusivity overcame one of the significant shortcomings of the GFDL constant diffusivity model: the absence of stratification below the thermocline. It was suggested that inappropriate specification of diapycnal processes is at least partly responsible for the incorrect abyssal stratification in that model. In the study of Cummins et al., the stratification dependent diffusivity led to only a small increase in the meridional overturning cell and to a decrease in the poleward heat transport with respect to the case of a small, constant diffusivity. Thus in the GFDL model, the strength and structure of the thermohaline circulation is dependent on the magnitude and depth dependence of the small-scale mixing processes.

Previous investigators' results do suggest a depth dependence of the diffusivity. Estimates of the vertical diffusivity from direct observation of dissipation in the thermocline are generally small, .1 $\mathrm{cm}^{2} / \mathrm{s}$ or less (e.g. Gregg and Sanford, 1988; Gregg, 1987; Gargett, 1989). Prior to the work reported herein, direct estimates at abyssal stratifications were virtually nonexistent. These direct estimates are derived from eddy diffusivity parameterizations of the mass $\left(\mathrm{K}_{\mathrm{p}}\right.$; Osborn, 1980) and heat $\left(\mathrm{K}_{\mathrm{T}}\right.$; Osborn and Cox, 1972) fluxes in turbulent production/dissipation balances. Isotropy and a constant ratio between the turbulent kinetic and potential energy fluxes are assumed in producing estimates of the rate of dissipation of turbulent kinetic energy $(\varepsilon)$ and rate of dissipation of thermal 
variance $(\chi)$ from estimates of the turbulent vertical shear and temperature gradient variances (see Appendix One for a further discussion). Estimates of a vertical diffusivity from heat budgets in closed basins using hydrographic data (e.g. Hogg et al., 1982; Johnson, 1990) or a combination of current meter and hydrographic data (e.g. Saunders, 1987; Whitehead, 1989; Barton and Hill, 1989) yielded values closer to $\mathrm{K}_{\rho} \sim 1-10 \mathrm{~cm}^{2} / \mathrm{s}$. These indirect estimates for the vertical diffusivity were based upon the flux of heat associated with flow below a given isotherm through a confined passage in which the bounding isotherm intersected the bottom within the basin beyond the passage. In such a case, the horizontal advection of heat is balanced by a diffusive flux through the bounding isotherm. The difference between direct estimates from the thermocline regions and indirect abyssal estimates implies a depth (or stratification) dependent diffusivity.

A number of ideas have been forwarded to explain this difference. Gargett and Holloway (1984) proposed that an increase in diffusivity with decreasing stratification at constant internal wave energy levels could be due a buoyancy scaling of the mechanisms associated with turbulent production. This speculation was based on direct estimates of the eddy diffusivity (Gargett and Osborn, 1981; Lueck et al., 1983) at thermocline stratification rates for data which lacked simultaneous estimates of internal wave energy levels. Later work by Gregg (1989) resulted in the claim that once the internal wave energy levels were accounted for, the eddy diffusivity would be independent of the stratification rate. However, the data in that 
analysis were said to lack sufficient variability in the stratification rate to support that claim (Gargett, 1990).

A distinct possibility which could explain the disparity between the abyssal heat budget estimates and the direct dissipation estimates from the thermocline is an increase in the internal wave energy levels at the boundaries of the ocean. Heightened internal wave energy levels might then lead to an increased diapycnal buoyancy flux at the boundary. Such boundary layers generate interior flows in a manner analogous to Ekman layer transports (see Garrett, 1991 for a review). The dynamical implications of this scenario for the general circulation are not obvious, however. Researchers (Rhines and MacCready, 1989; McDougall, 1989) have argued that the net effect of boundary mixing may result in interior abyssal circulations which may differ significantly from the broad poleward flow envisioned in Stommel and Arons (1960). Thus horizontal variations in the global patterns of mixing may have rather significant implications for large scale processes. An understanding of the dynamics of processes at small vertical scales and short time scales may be necessary to understand the dynamics of motions on a basin scale and the large scale thermodynamic structure of the oceans.

There exists a hierarchy of dynamical processes which govern the motions at various scales which are intimately related. The occurrence of three dimensional turbulence at scales of $\mathrm{O}(1 \mathrm{~cm})$ is generally held to result from the instability of motions (e.g. shear instability) on scales of several meters. The occurrence of shear instability results from the local intensification of the shear in the 
internal wave field. The energy dissipated in such events is provided by the energy flux to higher wavenumbers in the internal wave field due to nonlinear wave/wave interactions on scales of tens to hundreds of meters. In turn, the strength of the wave/wave interactions depends generally on the energy content of the internal wave field, which can vary considerably over even larger scales due to the presence of topography or background flows.

The prediction of global patterns of mixing caused by internal waves is a two fold problem. The first is the parameterization of turbulent mixing, or equivalently the rate of dissipation of turbulent kinetic energy, in terms of the background stratification rate, energy level, frequency content and vertical anisotropy / horizontal inhomogeneity of the internal wave field. The latter half of the problem is then to assess the climatology of the internal wave field: defining the efficacy of surface forcing and topographically related generation mechanisms (see Garrett and Munk (1979) for a review) and evaluating the ability of internal waves to interact with their environment to produce localized regions of heightened energy levels.

For most of the ocean the second portion of the problem is well defined. The energy content of motions with frequencies in the internal band is observed to be relatively constant, usually to within a factor of two, and this energy level generally exhibits a buoyancy scaling consistent with WKB theory (Munk, 1981). The frequency and wavenumber spectra are also observed to be remarkably uniform. Such observations led to the construction of the Garrett and Munk canonical spectrum (e.g. Munk, 1981; Garrett and Munk, 1972). 
This spectrum is an empirical result, assuming only linear internal wave dynamics and WKB scaling in the vertical. It provides no clues as to what the dynamics are which actually shape the spectra and why the energy levels and spectral shapes appear to be universal. Theoretical results from internal wave/wave interaction theory (e.g. McComas and Muller, (1981), Henyey Wright and Flatte (1986)) do, however, indicate that the Garrett and Munk spectrum is close to being statistically stationary with respect to the nonlinear interactions. Garrett and Munk (1979) present the following rationalization which they attribute to Cox and Johnson (1979, unpublished manuscript). If the time scale between internal wave generation events is smaller than the time scale to dissipate the energy received in a generation event, then the internal wave energy level will be fairly constant at a given location. Moreover, if the time scale for nonlinear interactions to redistribute the energy in the spectrum is small, then the change in the spectrum resulting from a generation event will quickly evolve to a spectrum which is in equilibrium with respect to the nonlinear interactions but at a higher energy level. Garrett and Munk then note that a fast time scale for nonlinear interactions to repartition energy within the spectrum does not necessarily imply a small time scale to dissipate that energy as those interactions may not result in a large upwavenumber energy flux at high wavenumbers. A dissipation time scale for the energy in the internal wave field on the order of a hundred days implies that the energetic low mode, low frequency component of the spectrum can spread by propagation to distances of $2000 \mathrm{~km}$ before being lost in a sea of nonlinear interactions. If such is the case, sources of 
internal waves would be fairly indistinct and Garrett and Munk note that spatially distant observations would tend to exhibit the same equilibrium spectral level.

There are, however, notable exceptions to the Garrett and Munk spectrum. Regions adjacent to sloping topography tend to exhibit heightened energy levels (Wunsch and Webb, 1979; Eriksen, 1982; Kunze et al., 1992). One possible cause is the enhancement of the small-scale internal wave variance due to internal wave reflections off a sloping bottom (Eriksen, 1985). Another possible cause is the generation of internal waves by the barotropic tide or mean flow over topography (Bell, 1975). While this topic is addressed only tangentially in the course of this study, the work herein has important implications for the topographically associated processes.

Regions of large background velocity are also generally associated with heightened internal wave energy levels and mixing (Kunze and Sanford, 1984; Kunze, 1986; Lueck and Osborn, 1986). Fronts and rings may act as scattering mechanisms due to the interaction of the mixed layer with the mean flow gradients and result in regions which are efficient generators of internal wave motions (e.g. Rubenstein and Roberts, 1986; Wang, 1991). The potential also exists that the internal waves interact with the gradients of larger scale flows in the interior (Kunze, 1985) to produce regions of heightened internal wave shear levels, causing enhanced mixing. Deep western boundary currents present potential critical layers for both freely (downward) propagating and upward propagating, topographically generated lee waves. This prospect 
raises the question of whether significant internal wave/mean flow interaction is associated with deep western boundary currents and whether such processes may also explain the disparity between direct and budget diffusivity estimates.

The relationships between the meso-, fine- and microscale fields in an upper ocean front is examined in Chapter Two.

Observations of internal waves in a background flow generally reveal heightened energy levels in regions of negative relative vorticity (Kunze and Sanford, 1984; Kunze, 1986). Kunze (1985) examines the problem of a single near-inertial wave interacting with a front in terms of a ray tracing argument. His study concludes that the lower bound for a freely propagating near-inertial internal wave is altered from $f$ to $f+\zeta / 2$ due to the presence of the background flow ( $\zeta$ is the relative vorticity and $f$ the Coriolis frequency). The ray tracing reveals these waves encounter horizontal turning points and vertical critical layers and are thus trapped to regions of negative relative vorticity. Heightened internal wave energy levels which are generally associated with regions of negative relative vorticity $(\zeta)$ in the background flow field are thus potentially due to internal waves with frequencies between $f+\zeta / 2$ and $f$. Waves with frequencies marginally above $f+\zeta / 2$ which propagate (vertically) into regions of more positive relative vorticity stall out as their frequencies become more inertial and their vertical group velocities become smaller. The dissipation of these waves is presumed to be associated with heightened mixing.

In the study of Kunze (1985) it was assumed that the waves originated at the surface with frequencies marginally above the local 
effective Coriolis parameter $(f+\zeta / 2)$. The problem of generation due to the interaction of the mixed layer with the front and subsequent propagation is more complicated because a range of frequencies is excited. Wang (1991) has examined the generation and subsequent propagation of internal waves in a baroclinic upper ocean front and finds internal wave energy levels a factor of four above background in the region of negative relative vorticity. In Chapter Two the pertinent mesoscale characteristics of an upper ocean front are discussed to provide a setting in which to interpret observations of internal wave variability. Estimates of the observed diffusivity are then related to variations of the energy content of the internal wave field. This study reveals the fine- and microstructure fields are well correlated, which in turn motivates the subsequent, more detailed parameterization studies in Chapters Three and Four.

The parameterization of turbulent mixing due to internal waves has been undertaken in the last decade in the context of a number of dynamical and kinematical models. These models generally produce estimates for the magnitude of the dissipation as a function of the internal wave energy level and the buoyancy frequency. A rather significant by-product of the parameterization studies is a greatly improved knowledge of internal wave dynamics. The dynamical models involve calculating the strength of the wave/wave interactions and ascertaining the average fluxes through frequency and wavenumber space. If the calculations indicate the spectrum which forms the basis for the analysis is statistically stationary with respect to the interactions, then the average upwavenumber flux can be equated with the rate of turbulent dissipation. Results from the 
dynamical models of McComas and Muller (1981) and Henyey, Wright and Flatte (1986) indicate that the Garret and Munk (GM; Munk, 1981) spectrum is stationary. The turbulent dissipation measurements therefore allow an assessment of both the vertical eddy diffusivity acting upon the background density profile and the strength of the nonlinear wave/wave interactions.

The issue of buoyancy scaling for the dissipation rate is examined in Section 3.5a using data which exhibited large variations in background stratification with depth and for which the internal wave characteristics were not substantially differentiable from the Garret and Munk presciption. The observation of GM characteristics and variable buoyancy frequency allows for an unambiguous assessment of the dynamical and kinematical models. A variety of non-GM environments are examined to ascertain the parameter dependence of $\varepsilon$ upon nonstationary characteristics in Section 3.5b. Such effects may be associated with the presence of a background flow, vertical anisotropy, horizontal inhomogeneity or deviations in the frequency and wavenumber spectra from the GM model. If one assumes that the model predictions are sufficiently stable with respect to perturbations in the internal wave frequency content, a frequency content scaling can also be obtained (Henyey, 1991). The influence of vertical anisotropy, horizontal inhomogeneity and the presence of a background flow upon interaction rates can not be simply addressed within the context of the dynamical models mentioned above (McComas and Muller, 1981; Henyey et al., 1986). Attempts at assessing the importance of a background flow (Muller, 1976) have not been successful to date (Ruddick and Joyce, 1979). 
One route by which kinematical models have been produced is through the statistics of instabilities which are thought to act on an intermediate scale, linking the wave/wave fluxes directly to the generation of turbulent events. With sufficient knowledge of the statistics and the dissipation produced in an event, this approach can also yield a parameterization for the magnitude of the average dissipation rate as a function of background stratification and internal wave energy and frequency content. A second kinematical approach involves scaling the production terms in a production/ dissipation balance. This treatment is more limited, providing only an energy level/stratification rate scaling.

It is generally assumed by researchers that some form of instability acts as an intermediary process in the upwavenumber transport between scales upon which wave/wave interactions dominate and those scales at which turbulence is found. This point of view forms the basis of Munk (1981) and Desaubies and Smith (1982). The resulting dissipation scaling of Munk is examined in Chapter Three. The linear shear instability mechanism appears as the basis of the dissipation scaling of Kunze, Williams and Briscoe (1990). Chapter Four examines the Kunze et al. scaling at great length. Theories have been forwarded (e.g. Smith et al., 1987) which suggest that the internal wave spectral shape and level at large wavenumbers is controlled by the turbulent momentum fluxes due to linear instability. Particular attention is paid to defining the possible length scales affected by a turbulent momentum flux due to shear instability. 
Chapter Three examines, in addition to the Munk model, predictions for the dissipation scaling from two dynamical models (McComas and Muller, 1981 and Henyey et al. 1986) and an additional kinematical one (Gargett and Holloway, 1984) and its extension by Gargett (1990). The two dynamical models differ significantly in their assumptions: the McComas and Muller model assumes the validity of weak wave/wave (triad) interaction theory whereas the Henyey et al. model is a ray tracing scheme for small scale waves in a background of larger scale internal waves. The models produce similar energy level and stratification scaling, but differ in their amplitude predictions and their frequency content scaling. Upon eschewing the usual Reynold's decomposition between waves and turbulence, Gargett and Holloway argue that the production terms in a production/dissipation balance should retain their internal wave scalings. This results in a dissipation scaling different than that of the dynamical models.

Oceanic profiles of temperature $(T)$, salinity $(S)$, the two components of horizontal velocity $(\mathrm{U}, \mathrm{V})$, the rate of dissipation of turbulent kinetic energy $(\varepsilon)$ and the rate of dissipation of thermal variance $(\chi)$ are used to examine the parameterization and internal wave interaction issues discussed above. These profiles were obtained with the High Resolution Profiler (HRP; Schmitt et al., 1988, Appendix One), a free falling, internally recording vertical profiler. The data examined herein were obtained during three separate field experiments. The first data set was obtained in conjunction with the Frontal Air-Sea Interaction EXperiment (FASINEX). This field work took place in February-March of 1986 in the vicinity of an upper 
ocean front in the sub-tropical convergence zone of the Northwest Atlantic $\left(29^{\circ} \mathrm{N}, 68^{\circ} \mathrm{W}\right)$. The front which was surveyed is one of a series of SW/NE oriented mid-ocean fronts which are believed to be associated with the confluence of Ekman transport in the sub-tropical gyre (Hanson et al., 1991). Additional vertical profiles of $\mathrm{T}, \varepsilon$ and $\chi$ from EPSONDE (Oakey, 1988), a loosely tethered profiler, are used to supplement the HRP FASINEX measurements. The second data set was obtained in March-April of 1990 from a warm-core ring of the Gulf Stream $\left(40^{\circ} \mathrm{N}, 64^{\circ} \mathrm{W}\right)$. The experiment is referred to herein as WRINCLE (the Warm Ring INertial Critical Layer Experiment). The third field experiment was in the vicinity of an isolated seamount (Fieberling Guyot; $32.5^{\circ} \mathrm{N}, 128^{\circ} \mathrm{W}$ ) $1000 \mathrm{~km}$ west of the Southern California coast in April of 1991 (TOPO). The seamount is located sufficiently far from the coast as to be outside the range of influence of the California Current. Large-scale background flows were observed to be less than $2 \mathrm{~cm} / \mathrm{s}$ at the time of the experiment ( $\mathrm{J}$. Toole, personal communication). The three cruises represent distinct geographic/oceanographic regimes. Note, however, that a bias towards late winter/early spring conditions exists.

This thesis consists of three main chapters. Evidence for the enhancement of internal waves due to wave/mean flow interaction processes is presented in Chapter Two, along with the corresponding variability in the mixing rates. Chapters Three and Four examine a number of dynamical and kinematical parameterizations of the turbulent dissipation rate due to the upwavenumber flux of energy associated with wave/wave interactions and the resulting generation of turbulence via linear the linear shear instability mechanism. 
Chapter Five provides a general summary of the major findings of Chapters 2-4 along with a concluding discussion of the implications this work has for determining the global patterns of mixing. Two appendices follow Chapter Five. The first discusses details of data processing common to the three main chapters. The second reviews the Garrett and Munk spectrum. 
24 


\section{Chapter Two}

Fine- and Microstructure Characteristics Across the Northwest Atlantic Subtropical Front

\subsection{Introduction}

Oceanic fronts, generally characterized by abrupt spatial changes of sea surface temperature (SST) and water mass characteristics aligned with a jet-like horizontal velocity field, are believed to be sites of enhanced mixing and dissipation. Conceptually, one might envision a down-scale cascade of energy: the presence of mesoscale features enhances 'large' scale internal wave variability, which in turn influences the finescale parameters which play a role in the production of turbulence. However, the relationships between the frontal fields with vertical scales of $100 \mathrm{~m}$. and horizontal scales of $10 \mathrm{~km}$. and the turbulence responsible for the mixing at the microscale, around $1 \mathrm{~cm}$., are not well understood. Previous studies suggested that internal waves could be trapped near (Kunze, 1985; Kunze and Sanford, 1984; Weller 1985; Mied et al., 1986), or preferentially associated with (Wang, 1991; Weller, 1982) a front. In turn, elevated internal wave shear levels are thought to be related to enhanced turbulent dissipation (Gregg et al., 1986; Marmarino et al., 1987; Gregg, 1989). Thus fronts are potentially important sites of oceanic mixing. In the following, we explore the relationship between the microstructure fields, the finescale internal wave velocity shear and an ocean front during Phase II 
of the Frontal Air-Sea Interaction EXperiment (FASINEX) using a series of vertical profiles of horizontal velocity and density obtained with the High Resolution Profiler (HRP) (Schmitt et al., 1988, Appendix One) and microstructure measurements from both the HRP and EPSONDE (Oakey, 1988).

Observations of internal waves in a background flow tend to reveal heightened energy levels in regions of negative relative vorticity (Kunze and Sanford, 1984; Kunze, 1986, Weller, 1985; Mied et al., 1986). Kunze (1985) examines the problem of a single nearinertial wave interacting with a front in terms of a ray tracing argument. The study concludes that the lower bound for a freely propagating near-inertial internal wave is altered from $f$ to $f+\zeta / 2$ due to the presence of the background flow ( $\zeta$ is the relative vorticity). The ray tracing reveals these waves encounter horizontal turning points and vertical critical layers and are thereby trapped to regions of negative relative vorticity. Waves with frequencies marginally above the effective $\mathrm{f}$ stall out as their vertical wavelength decreases, their intrinsic frequency becomes more inertial and their vertical group velocity becomes small as they propagate into regions of higher relative vorticity. Waves with frequencies slightly above the planetary $\mathrm{f}$ will not be trapped but can still interact strongly with the background flow. Heightened internal wave shear levels generally associated with regions of negative relative vorticity $(\zeta)$ in the background flow field are thus potentially due to internal waves with near-inertial frequency content. Heightened shear levels imply increased turbulent dissipation rates (Gregg, 1989), and these regions are presumed to be regions of heightened mixing. In the study of 
Kunze (1985) it was assumed that the waves originated at the surface with frequencies marginally above the local effective Coriolis parameter $(\mathrm{f}+\zeta / 2)$.

Near-inertial internal waves can also interact with a background flow through Doppler shifting (Kunze, 1985; Eriksen, 1988). Near-inertial internal waves propagating against (with) a region of increasing background flow encounter critical layers (turning points) as the wave's intrinsic frequency increases (decreases). Observations of near-inertial internal waves interacting with a background flow through the Doppler shift are discussed by Mied et al. (1987) and Mied et. al. (1990). Mied et al. (1990) suggest that the Doppler shifting mechanism may act to filter out all but cross-stream propagating waves and thus result in an inhomogeneous internal wave field.

The problem of generation due to the interaction of a surface mixed layer with the front and subsequent propagation is more complicated because a range of frequencies is excited. The generation of internal waves resulting from the interaction of the mixed layer with gradients of a background flow is most simply understood in terms of a slab mixed layer and a barotropic front (e.g. Rubenstein and Roberts, 1986). It is assumed that the passage of an atmospheric event results in the spatially uniform excitement of the mixed layer. An absence of spatial gradients in the mixed layer implies the initial frequency of oscillation is at the local inertial value, $f+\zeta / 2$. If the relative vorticity gradients are large or the spatial scales of the background flow and mixed layer depth are small, the mixed layer motions go quickly out of phase, leading to 
convergence and divergence within the mixed layer. The change in mixed layer depth with a near-inertial time scale is termed inertial pumping and leads to the generation of internal waves. The accumulation of spatial gradients in the time dependent flow implies an increase in the frequency content of the inertial motions, which in turn implies the radiation of internal waves into the interior (Rubenstein and Roberts, 1986). The process of internal wave generation may be further complicated by the presence of convergence (divergence) in the background flow which may lead to exponential growth (decay) of the mixed layer motions (Weller, 1982, 1985). A convergent background flow implies an increase in the amplitude of the forcing of internal waves via inertial pumping. For a given realization of the background flow, it is not apparent a priori that a significant amount of energy will be contained in waves with frequencies below f. Wang (1991) has examined the generation and subsequent propagation of internal waves in a baroclinic upper ocean front and finds internal wave energy levels a factor of four above background in the region of negative relative vorticity. Thus front may be regions of heightened mixing due to the increased efficiency of generation mechanisms.

Gregg (1989) has previously attempted to quantify turbulent dissipation rates as a simple function of the internal wave energy level and buoyancy frequency. That dissipation scaling was based upon dynamical models using the Garrett and Munk spectrum (GM; Munk, 1981) to calculate the average upwavenumber energy flux resulting from wave/wave interactions. These models equate the rate of turbulent dissipation with the average upwavenumber flux of 
energy in the internal wave dominated, low wavenumber $(.01-.1$ $\mathrm{cpm}$ ) portion of spectrum. We anticipate that a variety of non-GM conditions (e.g. the presence of a background flow, horizontal inhomogeneity, vertical anisotropy, and deviations from the GM frequency and vertical wavenumber spectral shapes) may alter the strength of the wave/wave interactions and therefore affect the mixing rates. We apply the model of Gregg (1989) to ascertain whether deviations of the internal wave characteristics associated with an upper ocean front quantitatively affect the mixing rates. In this chapter we wish to quantitatively examine the spatial variability of the intermediate vertical wavelength internal wave shear relative to an upper ocean front as a measure of the strength of the finescale activity and then relate the finescale shear variability to mixing intensity in the FASINEX work area. Chapter Two is organized as follows: The finescale sampling and background velocity structure is described in Section 2.2 and serves as the basis with which to interpret the horizontal variability of the internal wave field. Section 2.3 describes the analysis methods. The general character of the finescale velocity field is examined in Section 2.4 before a presentation of the shear statistics and their variation with respect to the background velocity structure of the front and with respect to time (Section 2.5). The mixing intensities are related to the finescale shear and the background velocity structure of the front in Section 2.6. Results and their implications are discussed in Section 2.7 . 


\subsection{Finescale Sampling and Background Velocity Structure}

A total of 38 HRP deployments were made within a span of 21 days during Phase II of FASINEX. Of these 38 profiles, 23 are examined herein. In the following, the variability of the internal wave field sampled by the profiler is examined relative to location with respect to the front. Seven of the profiles (8-10 and 22-25) were excluded from the analysis due to uncertainty in the spatial structure of the front and the location of the deployments relative to it; eight other deployments (1-3, 11, 18, and 37-39) were excluded for various instrumental reasons. HRP deployments in FASINEX typically occurred in groups of 2-5 and were often limited by other observational programs. Each group of profiles formed a quasisynoptic section across an upper ocean front. The 23 deployments represent four surveys of the FASINEX front.

The profiler surveys were further interrupted by a series of atmospheric events (storms) of 1-2 day duration. At several points during the experiment wind speeds reached $15 \mathrm{~m} / \mathrm{s}$ or larger. However, no rapid clockwise rotation (i.e. inertial period or less) of the wind direction was in evidence during the field program (Phase II). Thus the wind forcing was not resonant with the near-inertial wave field.

HRP deployments 29-33 constituted a representative section across the fronts sampled during FASINEX, Figures 2.1 and 2.2. The potential temperature distribution revealed an upper ocean front with its surface expression resulting from the outcropping of isotherms near $22^{\circ} \mathrm{C}$ (Figure 2.1). The along-front velocity field, 


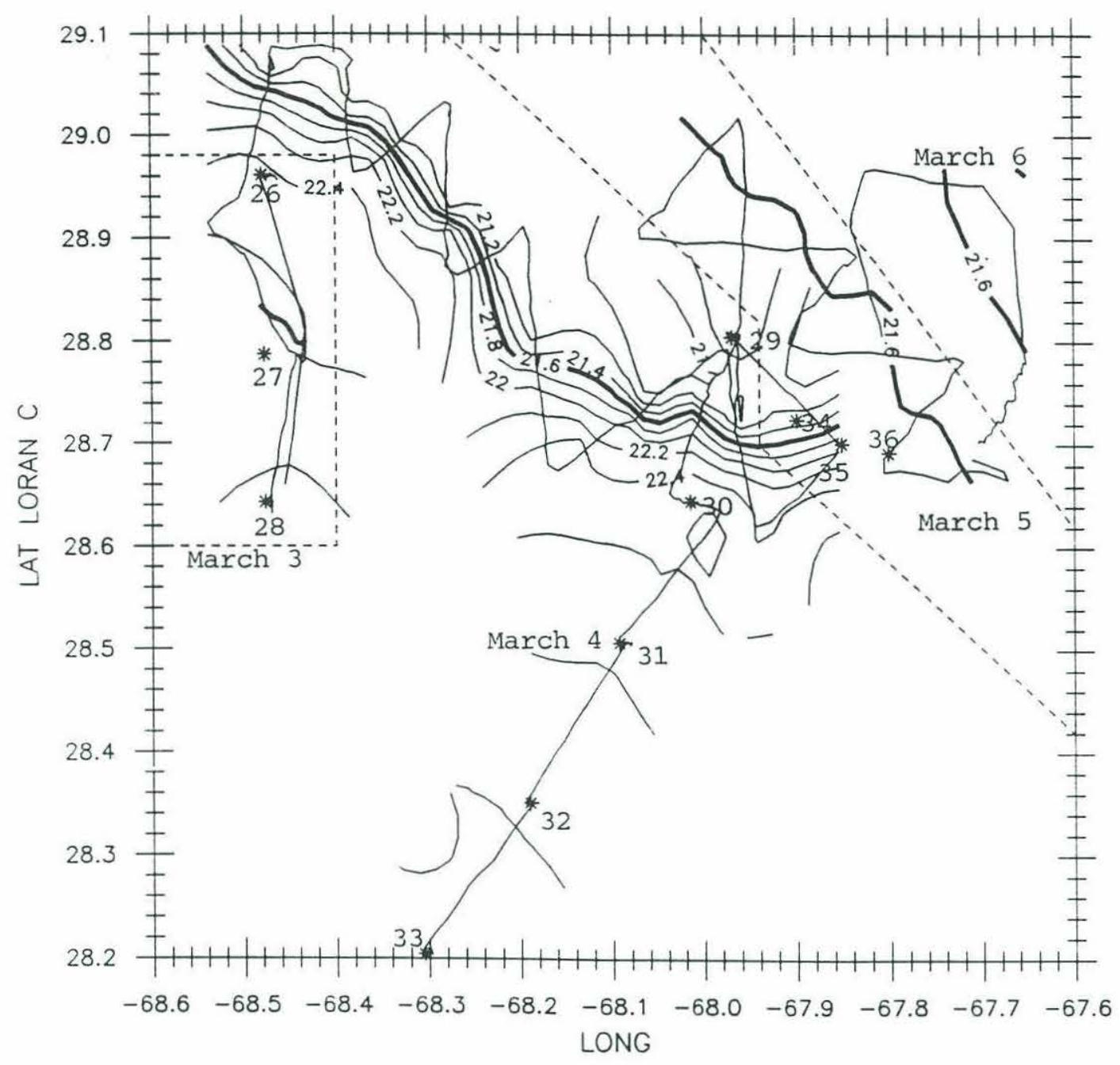

Fig. 2.1: Contours of SST for selected intervals between March 3 and 6,1986 . Over-plotted are the ship tracks and positions of the profiler deployments for this time period. Note that this plot includes four realizations of the front, and that the front is seen to move quite rapidly from West to East. The contours for three of these (March 3, 5 and 6) have been replaced by a single contour at $21.6^{\circ} \mathrm{C}$. 


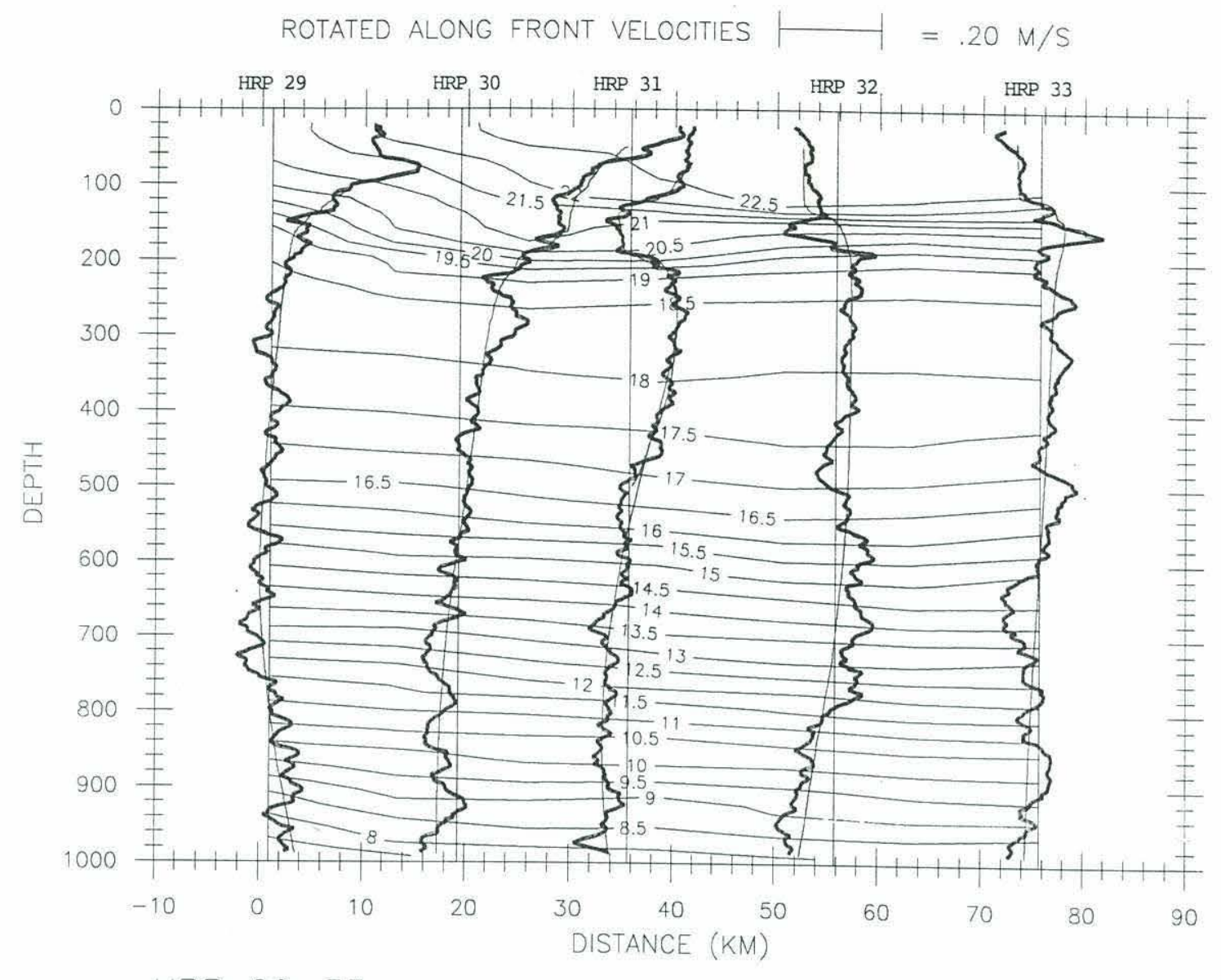

HRP 29-33

Fig. 2.2: Velocity profiles (along-front component) and contours of potential temperature from profiler deployments 29 (left) to 33 (right) (see also figure 1) The potential temperature distribution reveals an upper ocean front with its surface expression at about $10 \mathrm{~km}$. Over-plotted on the profiler velocities (thick) are low order polynomial fits to the velocity vs sigma-theta (thin). The straight vertical lines represent the vertical axes of the respective profiles and are displaced horizontally from each other an amount proportional to their distance from the northern most profile (profile 29). The velocity profiles are not absolute and have been centered such that there is zero net flow between 250 and $1000 \mathrm{db}$. 
obtained directly with the HRP, was characterized by a surfaceintensified jet roughly centered about the surface temperature expression, Figure 2.2, consistent with the thermal wind balance. Negative (positive) relative vorticity was noted to the south (north) of this surface expression. Just on the warm side of the front (profiles 30 and 31 ) was a $21^{\circ} \mathrm{C}$ thermostad which Pollard (1986) suggested was subducted surface waters from the cold side of the front. The along-front velocity profiles also had low values of background shear within the $21^{\circ} \mathrm{C}$ thermostad, a pronounced reversal in vertical shear at the edge of the thermostad (HRP 31 around $200 \mathrm{~m}$ depth), and the small westward velocities well south of the front. As evidenced by Figure 2.2, the presence of the $21^{\circ} \mathrm{C}$ thermostad and associated flow field significantly complicated the background horizontal shear field in the upper ocean; relative vorticity appeared to change sign at the southern edge of the thermostad.

Figures 2.3 and 2.4 are used to illustrate the velocity structure in greater detail. The data in Figures 2.3 and 2.4 come from a CTD Tow-Yo station across another section of the front on February 23, or nine days prior to the section shown in Figure 2.2. In Figure 2.3 contours of potential density and geostrophic velocity estimates relative to $385 \mathrm{db}$. are presented. In Figure 2.4, contours of relative vorticity derived from the geostrophic estimates are presented with contours of potential density superimposed. The background velocity characteristics noted in Figure 2.2 are apparent in Figure 2.3 and 2.4: Associated with the rising of the isopycnals to the surface is an eastward directed jet. The velocity maximum is generally to the south of the main SST gradients. To the north is a region of positive 


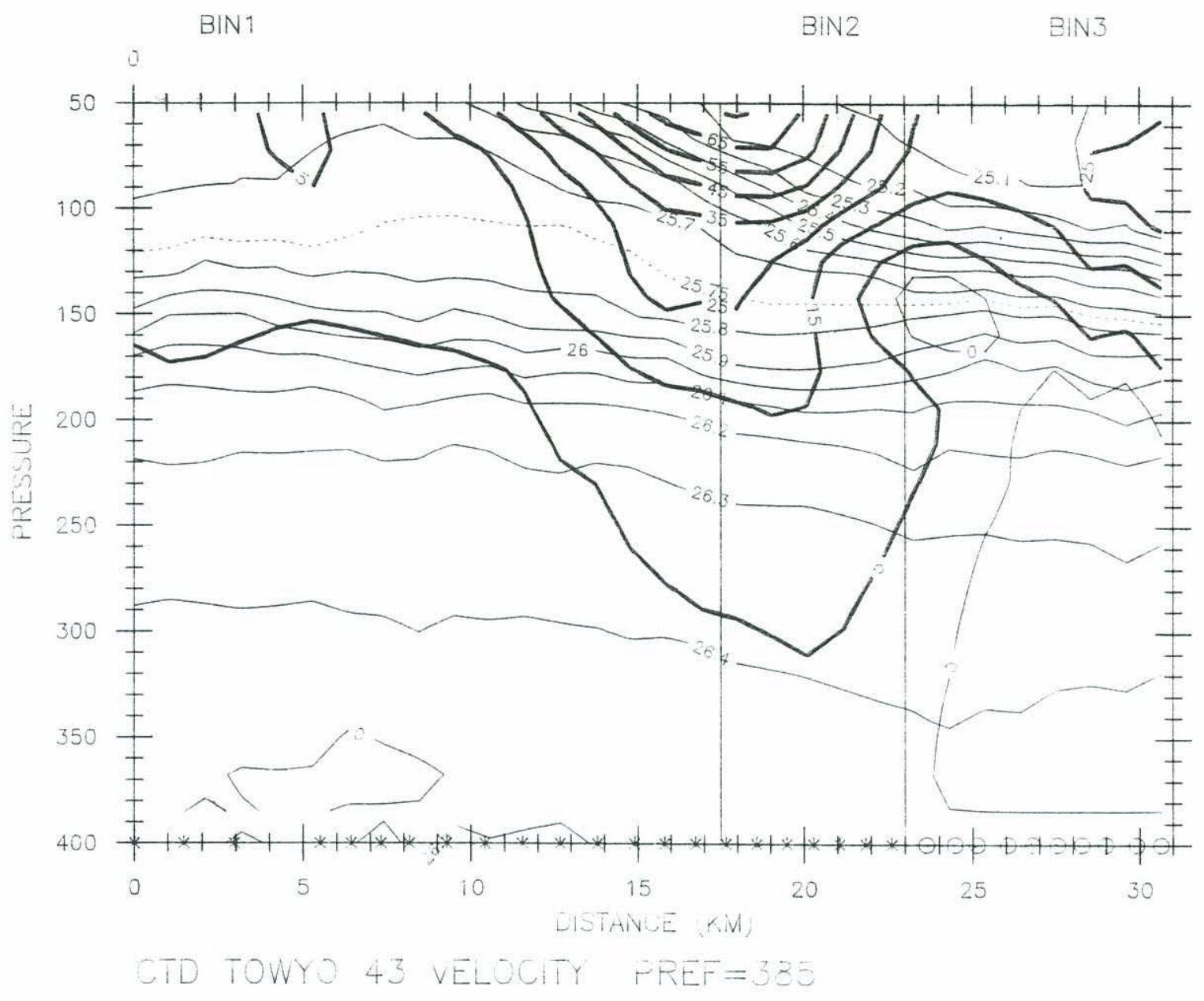

Fig. 2.3: Contours of potential density and geostrophic velocity relative to $385 \mathrm{db}$. from a CTD Tow-Yo section obtained on February 23, 9 days prior to the HRP section in Figure 2.2. Bins 1-3 are deliniated by vertical lines at 17.5 and $23 \mathrm{~km}$. and denote regions of distinct relative vorticity characteristics (Section 2.3b). 


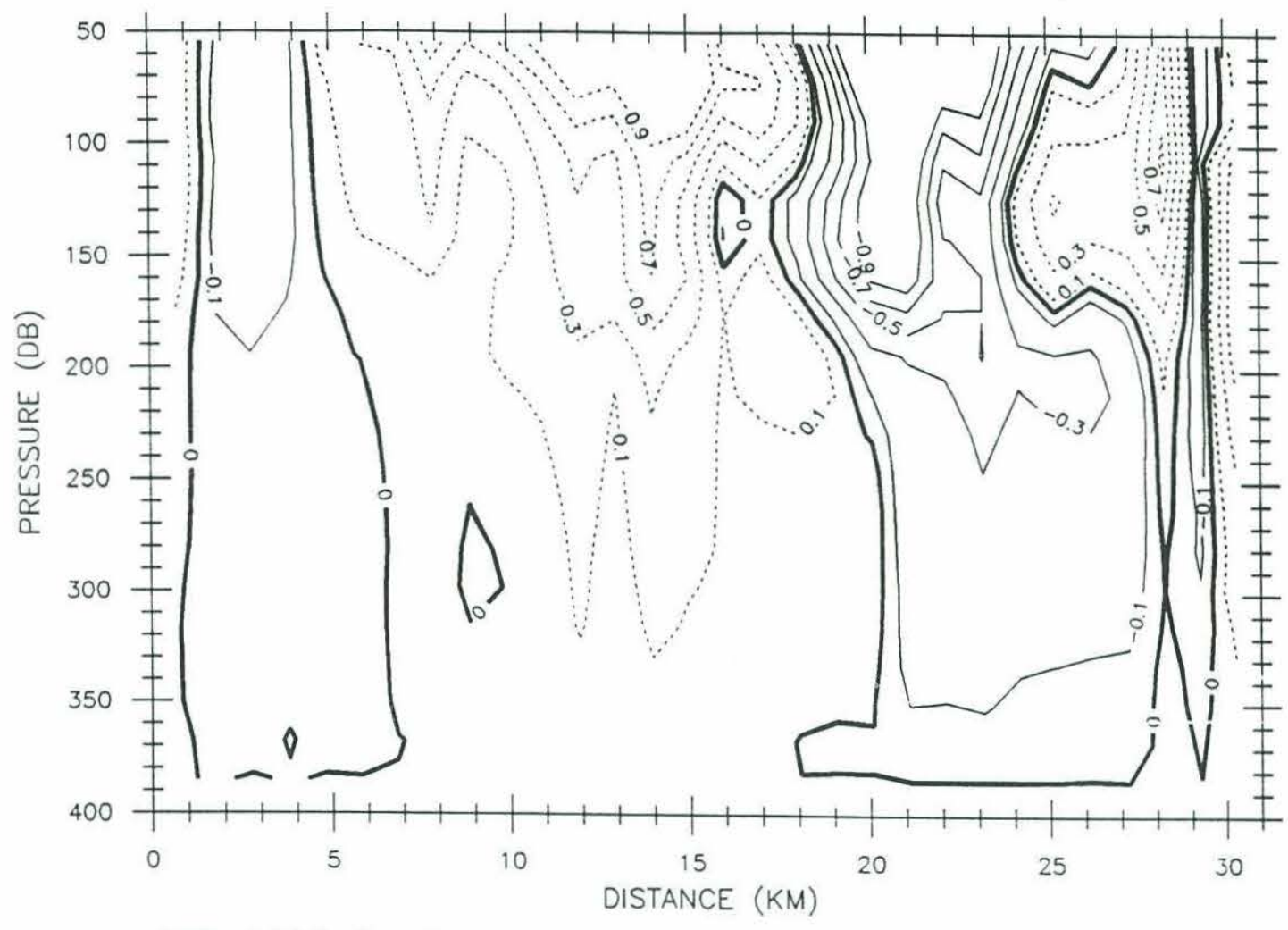

CTD TOWYO 43 RELATIVE VORTICITY

Fig. 2.4: Contours of relative vorticity divided by the Coriolis parameter $(\zeta / f)$ derived from the geostrophic velocity estimates presented in Figure 2.3. The contour interval is 0.2 with additional zero contours. Dashed (solid) lines imply positive (negative) values of the relative vorticity, respectively. 
relative vorticity, to the south the relative vorticity is seen to be negative. These regions of positive and negative relative vorticity are delineated by solid vertical lines in Figures 2.3 and 2.4. A region of relatively small westward geostrophic velocities is found at a distance of approximately $25 \mathrm{~km}$ in Figure 2.2 and is associated with the pycnostad at $125 \mathrm{db}$. Negative relative vorticities were found north of $25 \mathrm{~km}$. To the south, a region of smaller positive relative vorticity was found. This change in relative vorticity south of the surface front was a recurrent feature in the data. The southward extent of the region of negative relative vorticity within the pycnostad corresponded quite dramatically with a salinity front of .03 psu on a potential density surface of $\sigma_{\theta}=25.75$. The presence of the relative vorticity and salinity fronts is designated by a second vertical line. 


\subsection{Analysis Methods}

A) Separation of Wave/Mean flow

Separation of the internal wave contribution to the oceanic shear field from the "background" flow field is problematic in these profile data; we did not have sufficient temporal information to do a frequency decomposition. We therefore invoked a scale separation to distinguish between waves and background flow by arguing that internal waves dominated the velocity field at small scales.

Velocity components were first transformed from east and north coordinates to along-front and across-front coordinates, as determined by the surface temperature distributions at the time of each HRP deployment (e.g. Figure 2.1). A "background" velocity profile was then defined by linear, low order polynomial fits (shown as the smooth profiles in Figure 2.2) to the along-front and acrossfront velocities as functions of potential density. The use of potential density as the independent variable was found necessary to adequately describe the vertical structure of the background flow. Geostrophic velocities were computed for the section. While the polynomial fits contained somewhat greater structure in the vertical, the differences were qualitatively minor and could be accounted for by the fact that the geostrophic velocities represent a spatial average of the velocities between station pairs. The small-scale internal wave velocities were then defined as the difference between these polynomial curves and the full profiles. Low order polynomials in pressure coordinates have been used by previous investigators (e.g. Kunze, 1986) to separate internal waves from background flows 
under the assumption that the background flow consists of a predominantly low-mode character. Invoking a scale separation to extract the internal wave component from profile data is, of course, artificial. Low-mode (long vertical wavelength) waves that can provide the largest contribution to internal wave velocity were removed by our procedure. The present study focuses on the shear field, which is expected to be dominated by internal wave motions at small scales (Kunze and Sanford, 1991; D’Asaro and Morehead, 1991). However, in the absence of any time domain information, this assumption cannot be verified.

A statistical study of this method of filtering out the larger scale velocity field was conducted by taking a polynomial fit for a profile within the frontal region and adding a synthetic Garret and Munk-like (Munk, 1981) internal wave profile. The horizontally homogeneous, vertically isotropic synthetic wave field was assumed to WKB scale and was defined as a sum of randomly phased propagating waves with wavelengths from 4000 to $2 \mathrm{~m}$. The synthetic field had a GM vertical wavenumber shear spectrum up to $.1 \mathrm{cpm}$ which fell with a slope of -1 thereafter (Appendix Two). The synthetic wave-polynomial fit profile was then analyzed in a manner identical to the procedure for the data described herein. Two dissimilar polynomial fits were analyzed for 20 realizations of the synthetic wave field. The variance in wavelengths smaller than about $500 \mathrm{~m}$ were not significantly affected by the filtering; amplitudes at $512 \mathrm{~m}$ wavelength were reduced by half, while those at $256 \mathrm{~m}$ were within $10 \%$ of the input spectrum. The ratios between the input synthetic upward and downward propagating 
spectra and the along-front and across-front spectra were also varied. A synthetic spectrum for which the $\mathrm{CCW}$ and across-front variance were twice the $\mathrm{CW}$ and along-front (both specified as GM) was analyzed in order to examine the impact of the fitting procedure upon the anisotropy and inhomogeneity signal. The fitting procedure was found to marginally degrade the phase information between the horizontal velocity components which is required to define the $\mathrm{CW}$ and CCW components. When analyzed in the same manner as the actual data, the ratio between the upward vs downward spectra was found to vary by less than $10 \%$ from its specified value (2) for wavelengths smaller than $500 \mathrm{~m}$. The ratio between the across-front and along-front propagating spectra decreased by less than $5 \%$ after the analysis. We conclude that representative estimates of the small-scale variability can be constructed by subtracting the polynomial velocity profiles from the original records.

\section{B) Binning}

Previous investigators suggested that the characteristics of the internal wave field are strongly modulated in space (Kunze and Sanford, 1984; Weller, 1985; Mied et. al., 1986) by the presence of an along-front jet-like flow field. Kunze (1985) and Weller (1982, 1985) have ascribed the variability as being associated with the relative vorticity field of the front and/or divergent background flow associated with the front. In order to document spatial variability of the small-scale internal wave field, the data were subdivided into five bins defined by position relative to the front. 
The first horizontal bin is defined as those profiles (5) located on the cold side of the front to the north of the surface velocity maximum. So defined, bin 1 was a region of positive relative vorticity (see Figures 2.3 and 2.4). Bin 2 contained profiles (8) taken just to the south of the surface velocity maximum, on the warm side of the front. This was a region of negative relative vorticity. Bin 3 consisted of four profiles in a region of positive relative vorticity associated with the $21^{\circ} \mathrm{C}$ thermostad. The distinction between bins 2 and 3 was made unambiguous due to the presence of the salinity front on $\sigma_{\theta}=25.75$ (Figure 2.3). Bins 1,2 and 3 are delineated by vertical lines in Figures 2.3 and 2.4). Bins 4 and 5, encompassing three profiles apiece, were in the far field of the front. Bin four ranged 30 to $60 \mathrm{~km}$ south of the surface expression of the front in a region of weakly negative relative vorticity. Bin five profiles were obtained near the moored array, $150 \mathrm{~km}$ south of the front (Weller et al., 1985). In Figure 2.2, profile 29 corresponds to bin 1, profile 30 to bin 2, profile 31 to bin 3 , and profiles 31 and 32 to bin 4 .

The profiles were further binned in the vertical in order to examine any depth or buoyancy frequency variability in the wave characteristics. The shallowest bin, $125-250 \mathrm{~m}$, was in all cases deeper than the mixed layer and extended through the seasonal thermocline to the top of the $18^{\circ} \mathrm{C}$ water. The middle bin, 250-450m, encompassed the $18^{\circ} \mathrm{C}$ water exclusively. The deepest bin in the vertical, 500 to approximately $1000 \mathrm{~m}$, we denote here as the main thermocline. 
C) Spectra

Small scale internal wave shear statistics are presented here in vertical wavenumber space in order to examine the vertical phase propagation characteristics of the internal wave field. Prior to Fourier transforming, each velocity profile was rotated, a background velocity in both along-front and across-front components was subtracted, and the profiles were then subjected to a WKB-stretching of the vertical coordinate $\left(\mathrm{N} / \mathrm{N}_{\mathrm{o}}\right)$ and $\mathrm{WKB}$-normalization of velocity $\left(\mathrm{N}_{\mathrm{o}} / \mathrm{N}\right)^{1 / 2}$ with respect to the buoyancy frequency profile, referenced to $\mathrm{N}_{\mathrm{o}}=3 \mathrm{cph}$ (Leaman and Sanford, 1975). The WKB scaling renders the velocity profiles more homogeneous in the vertical by removing variations in amplitude and wavelength due solely to variations in the background stratification. Since the buoyancy profile varied greatly from one HRP deployment to the next in the upper ocean, the background stratification was defined by a linear fit to individual profiles of potential density. The vertical scale of this fit was $25 \mathrm{~m}$ from the surface to $200 \mathrm{db}$, where upon it was gradually increased to $100 \mathrm{~m}$ at $250 \mathrm{db}$. This sliding scale was employed in order to minimize contamination of the background $\mathrm{N}^{2}$ estimates due to internal wave straining below the seasonal thermocline and yet enable the fit to capture the obvious variability related to the presence of the $21^{\circ} \mathrm{C}$ thermostad. A simple calculation based on the GM displacement spectrum (Munk, 1981) suggested that the $25 \mathrm{~m}$ fit employed in the seasonal thermocline should filter out all but a $5 \%$ variability in the buoyancy frequency due to internal wave straining. The resulting profiles were then fit with a spline and resampled at 
evenly spaced pressure intervals to be compatible with a fastFourier transform procedure.

Stretched and normalized profiles were subsequently detrended, prewhitened, and $10 \%$ cosine tapers employed before Fourier transforming; each transformed estimate was then recolored with the theoretical inverse of the pre-whitening filter. Finally, shear spectra were generated by multiplying velocity spectral density estimates by wavenumber squared and corrected for the loss of variance due to the windowing process. All confidence intervals displayed here were calculated using a reduced number of degrees of freedom which results from the the tapering (Persson, 1974). Rotary spectral estimates (clockwise denoted by $\mathrm{CW}$ and counter clockwise by $\mathrm{CCW}$ ) were formed from a linear combination of the across-front (rotated north, v) and along-front (rotated east, u) Fourier coefficients (Gonella 1972, Mooers 1973).

\subsection{General character of finescale velocity}

\section{A) $\mathrm{CW}$ vs $\mathrm{CCW}$ signal in velocity}

As described by Leaman and Sanford (1975), clockwise rotation of the velocity vector with depth is a signature of downward energy propagation in the near-inertial internal wave field. The ensemble-averaged rotary velocity spectral density estimates for the full data set (Figure 2.5) show elevated levels of CW energy density relative to the $\mathrm{CCW}$ component. Also shown in Figure 2.5 is the onecomponent canonical spectral density prediction of Munk (1981) (the model is vertically isotropic and horizontally homogeneous) scaled 


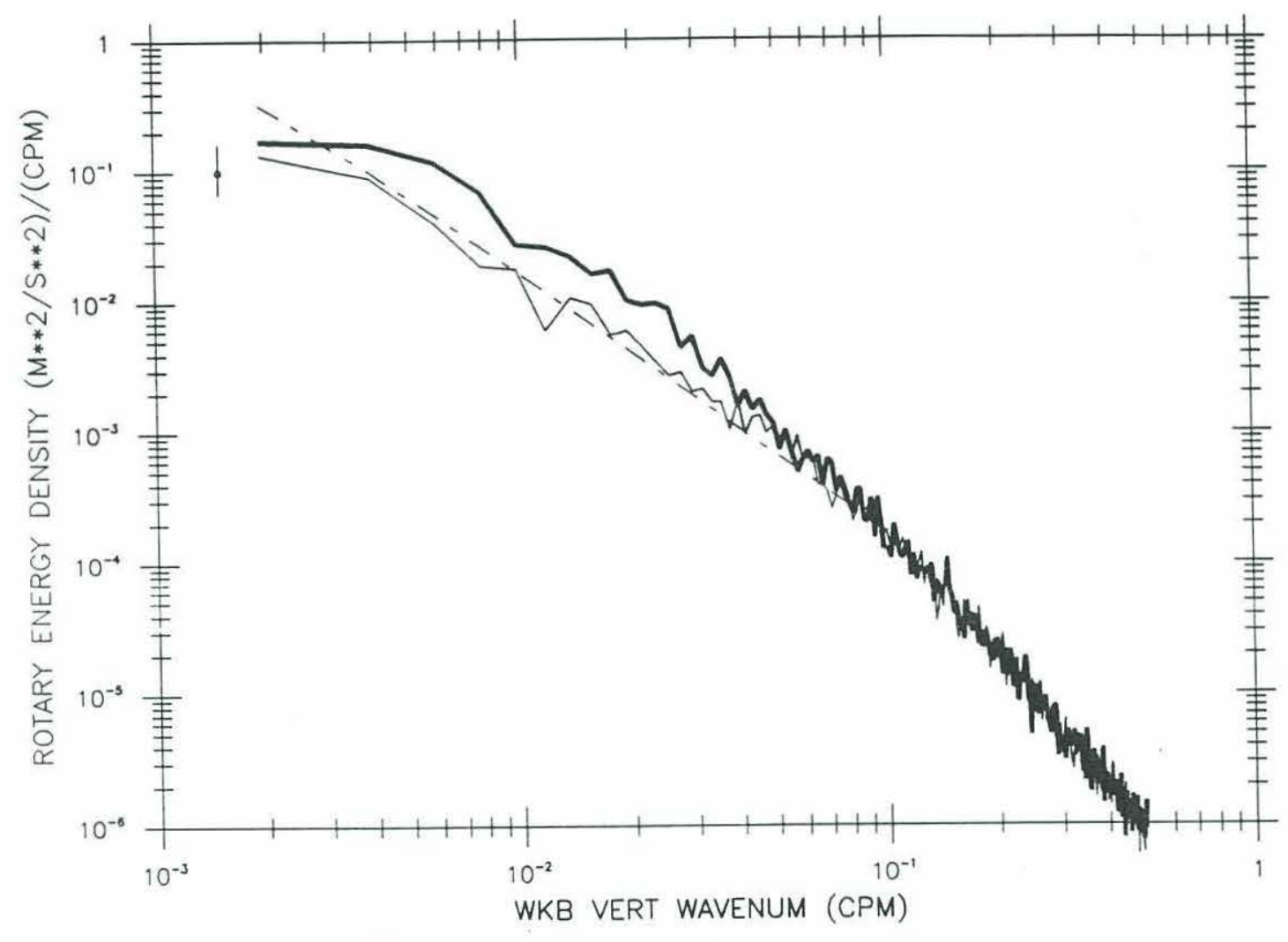

WKB CW CCW ALL FIRST 512 STR M

Fig. 2.5: WKB rotary velocity spectra [thick $(\mathrm{CW})$, thin $(\mathrm{CCW})$, dashed $(\mathrm{GM})]$ for the first 512 stretched meters starting from $125 \mathrm{db}$. averaged over the data set. The largest differences between $\mathrm{CW}, \mathrm{CCW}$, and GM occurred in the interval .0078 to $.031 \mathrm{cpm}$ (128 to 32 stretched meters, Nref $=3 \mathrm{cph}$ ). No band averaging has been employed. Ninety-five percent confidence levels are shown to the left. 
with the 3 -cph reference buoyancy frequency. The intermediate wavelength $\mathrm{CW}$ spectral estimates were in general a factor of two larger than the GM spectra, a difference significant at the $95 \%$ confidence level. In contrast, $\mathrm{CCW}$ estimates were not statistically different $(95 \%)$ from the GM spectra. Thus the small-scale internal wave field appeared on average to be transporting energy downward during Phase II. In the absence of additional information about wave characteristics (i.e. frequency and horizontal wavenumber) we were unable to quantify the flux (e.g. D'Asaro and Perkins, 1984). A notable feature of these rotary spectra was their flattening at scales longer than about 250 stretched meters. Based on the statistical study mentioned earlier, the decrease of the spectra due to the fitting procedure was responsible for a factor of two decrease only at the longest wavelength, so the drop of the spectra to or below GM levels must reflect the absence on average of persistent, energetic, large-scale internal waves in the data set, in contrast to the observations of Kunze and Sanford (1984) from the Pacific Subtropical Convergence.

The structure of these velocity spectra motivated our use of the statistic "shear spectral density between $0.0078(128 \mathrm{~m})$ and 0.31 $(32 \mathrm{~m}) \mathrm{cpm} "$ to examine the space/time variability of the small-scale internal wave field. This quantity will henceforth be referred to as $\mathrm{S}_{\mathrm{z}}$. The choice of a maximum wavelength of $128 \mathrm{~m}$ also allows partitioning of the vertical profile into the three depth bins defined earlier (125-250, 250-450 and 500-1000 m). 
B) WKB scaling of shear variance

The ensemble averaged, WKB-scaled shear spectral density estimates differed somewhat from the presentation of Gargett et al. (1981), whose canonical spectrum is also shown in Figure 2.6. They identified a break in the slope of the shear spectral energy density from white to -1 at a constant (non-stretched) wavenumber of $.1 \mathrm{cpm}$. The averaged spectra in Figure 2.6 suggest a slightly greater high wavenumber slope (see also Kunze, 1990 and Section 4.5). Here the cut-off wavenumber appears at different stretched wavenumbers in the three plots. Gargett et al. choose to Fourier transform and then scale the shear spectral density estimates by $\mathrm{N}^{2}$. Their estimates are displayed with respect to an unscaled vertical wavenumber. Here we have scaled, stretched, and then transformed. In this process, the constant $.1 \mathrm{cpm}$ break in slope is shifted along the horizontal axis. Researchers have previously argued that the cutoff wavenumber is inversely dependant upon the average shear spectral density (Munk, 1981; Duda and Cox, 1989; Gargett 1990; see also Section 4.5 and Appendix 2). With the stretching procedure employed here, this aspect cannot be addressed.

The largest differences between $\mathrm{CW}$ and $\mathrm{CCW}$, and $\mathrm{CW}$ and $\mathrm{GM}$ values occur in the seasonal thermocline, consistent with wave generation near the surface and downward energy propagation. The WKB scaling accounts for most, but not all, of the vertical variation in $S_{z}$. Horizontally averaged values of $S_{z}$ over all profiles for each depth bin are presented in Table 2.1. In each depth bin, the CW estimate was significantly larger $(95 \% \mathrm{ci})$ than the $\mathrm{CCW}$. The $\mathrm{CW}$ estimate in the seasonal thermocline was larger than the $\mathrm{CW}$ estimates in either 


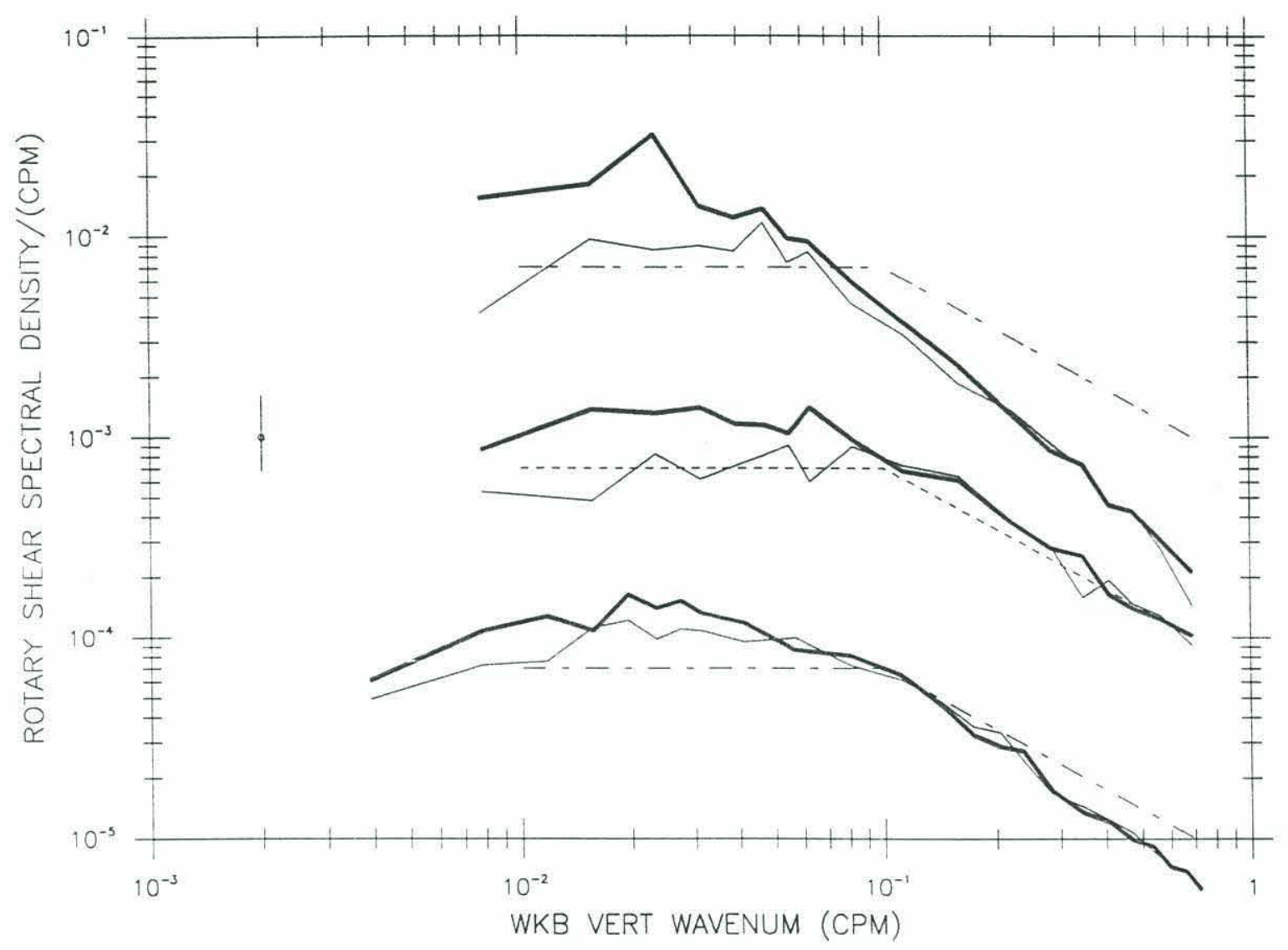

WKB CW CCW

Fig. 2.6: WKB-scaled Rotary shear spectral density averaged over the data set for each depth interval. (thick CW, thin CCW, dashed GM/Gargett et al.) From the top: 125-250m, 250-450m, and 500$1000 \mathrm{~m}$. Average buoyancy frequencies are $4.0,1.9$, and $2.5 \mathrm{cph}$, respectively. The upper graphs are offset sequentially by one decade. Included for each pair is half the GM shear spectral level for wavenumbers less than $.1 \mathrm{cpm}$, falling off as $\mathrm{m}^{-1}$ at smaller wavelengths. Two overlapped sections of 256 stretched meters were used for the main thermocline data and accounts for the extra spectral estimate at low wavenumbers. 
the eighteen degree water or the main thermocline, while the eighteen degree water and main thermocline $\mathrm{CW}$ estimates were numerically similar (1.31 vs $\left.1.33 \times 10^{-4} \mathrm{~s}^{-2} / \mathrm{cpm}\right)$ Counter clockwise estimates were distinguishable only in that the $S_{z}$ estimate in the main thermocline was larger than in the eighteen degree water. Note that the ratio of $\mathrm{CW}$ to $\mathrm{CCW}$ shear is found to decrease monotonically with depth.

Table 2.1: WKB shear spectral densities $\times 10^{-4} \mathrm{~s}^{-2} / \mathrm{cpm}, .0078$ to .031 $\mathrm{cpm}$. Estimates are given for rotary and total components for each depth and horizontal bin. The ' $\Sigma$ ' signifies the profile averaged spectral densities for each depth bin. Also included are $95 \%$ confidence intervals in the format:

lower bound I estimate I upper bound.

\begin{tabular}{ccc|c|c|c|cc|c|c}
$125-250 \mathrm{db}$ & \multicolumn{2}{c}{$\mathrm{CW}$} & \multicolumn{2}{c}{$\mathrm{CCW}$} & \multicolumn{3}{c}{$\mathrm{CW}+\mathrm{CCW}$} \\
\hline \hline Bin $(1)$ & 1.15 & 1.74 & 2.94 & .50 & .75 & 1.27 & 1.85 & 2.50 & 3.85 \\
$(2)$ & 2.17 & 2.94 & 4.38 & .69 & .96 & 1.43 & 3.04 & 3.90 & 5.15 \\
$(3)$ & 0.35 & .56 & 1.01 & .45 & .71 & 1.29 & 0.91 & 1.27 & 1.89 \\
$(4)$ & 1.48 & 2.47 & 4.94 & .35 & .58 & 1.16 & 2.08 & 3.06 & 4.93 \\
$(5)$ & $\underline{0.82}$ & 1.36 & 2.72 & .39 & .65 & 1.30 & 1.37 & 2.02 & 3.25 \\
$(\Sigma)$ & 1.64 & 1.99 & 2.51 & .64 & .78 & .98 & 2.38 & 2.77 & 3.24
\end{tabular}

\begin{tabular}{rrr|r|r|r|rr|r|r}
$250-450$ & $\mathrm{db}$ & \multicolumn{1}{c}{$\mathrm{CW}$} & \multicolumn{3}{c}{$\mathrm{CCW}$} & \multicolumn{3}{c}{$\mathrm{CW}+\mathrm{CCW}$} \\
\hline \hline Bin $(1)$ & 1.04 & 1.57 & 3.26 & .52 & .79 & 1.34 & 1.75 & 2.36 & 3.37 \\
$(2)$ & 1.05 & 1.46 & 2.18 & .52 & .72 & 1.07 & 1.57 & 2.18 & 2.88 \\
$(3)$ & .72 & 1.15 & 2.08 & .32 & .51 & .92 & 1.20 & 1.66 & 2.47 \\
$(4)$ & .54 & .90 & 1.80 & .46 & .76 & 1.52 & 1.13 & 1.66 & 2.67 \\
$(5)$ & $\underline{0.31}$ & .51 & 1.02 & .55 & .92 & 1.84 & 0.97 & 1.43 & 2.30 \\
$(\Sigma)$ & 1.07 & 1.31 & 1.64 & .52 & .64 & .81 & 1.68 & 1.95 & 2.28
\end{tabular}

\begin{tabular}{cc|c|cc|c|cc|c|c}
$500-1000 \mathrm{db}$ & \multicolumn{1}{c}{$\mathrm{CW}$} & \multicolumn{2}{c}{$\mathrm{CCW}$} & \multicolumn{3}{c}{$\mathrm{CW}+\mathrm{CCW}$} \\
\hline \hline Bin $(1)$ & 1.24 & 1.59 & 2.11 & 1.13 & 1.45 & 1.93 & 2.51 & 3.03 & 3.70 \\
$(2)$ & 1.20 & 1.46 & 1.83 & .85 & 1.04 & 1.30 & 2.17 & 2.49 & 2.91 \\
$(3)$ & 0.91 & 1.20 & 1.66 & .79 & 1.04 & 1.44 & 1.83 & 2.23 & 2.79 \\
$(4)$ & 0.82 & 1.12 & 1.64 & .31 & .43 & .63 & 1.24 & 1.55 & 2.02 \\
$(5)$ & $\underline{0.64}$ & .87 & 1.27 & .49 & .67 & .98 & 1.22 & 1.53 & 1.99 \\
$(\Sigma)$ & 1.18 & 1.33 & 1.52 & .89 & 1.00 & 1.14 & 2.14 & 2.33 & 2.54
\end{tabular}




\subsection{Finescale variability vs Frontal position}

\section{A) Rotary estimates}

The largest degree of spatial variability in $S_{z}$ appears in the seasonal thermocline (Figures 2.7 and 2.8) and was correlated with the velocity structure of the front. In no bin were the CCW estimates statistically different from GM values, whereas in all but the third bin (i.e. the bin associated with the region of positive relative vorticity south of the front) the $\mathrm{CW}$ estimate was significantly larger at the $95 \%$ confidence levels than GM spectral levels. In all regions except bin three, a factor of two to four difference in $\mathrm{CW}$ vs $\mathrm{CCW}$ shear statistic was noted. These $\mathrm{CW}$ vs $\mathrm{CCW}$ differences were largest and statistically significant for those regions associated with negative relative vorticity of the background flow (i.e. bins 2 and 4).

Moreover, a statistical difference in $\mathrm{CW}$ shear was observed between the average shear in bins $1,2,4$ and that in bin 3 . The total shear statistic $(\mathrm{CW}+\mathrm{CCW})$ has the same trends as the $\mathrm{CW}$ statistic alone (Table 2.1).

B) Across-front / Along-front anisotropy

A strong degree of internal wave anisotropy was noted in regions of large background shear (i.e. bins 1-3 for the depth range 125-250 db., Figure 2.8). Averaging the shear spectra for bins 1-3 and bins 4-5 resulted in a general pattern (i.e. across-front shear tending to be larger than the along front shear) at the largest wavelengths in the analysis for the shear spectral density of the former, but not the later. A comparison between the along/across- 


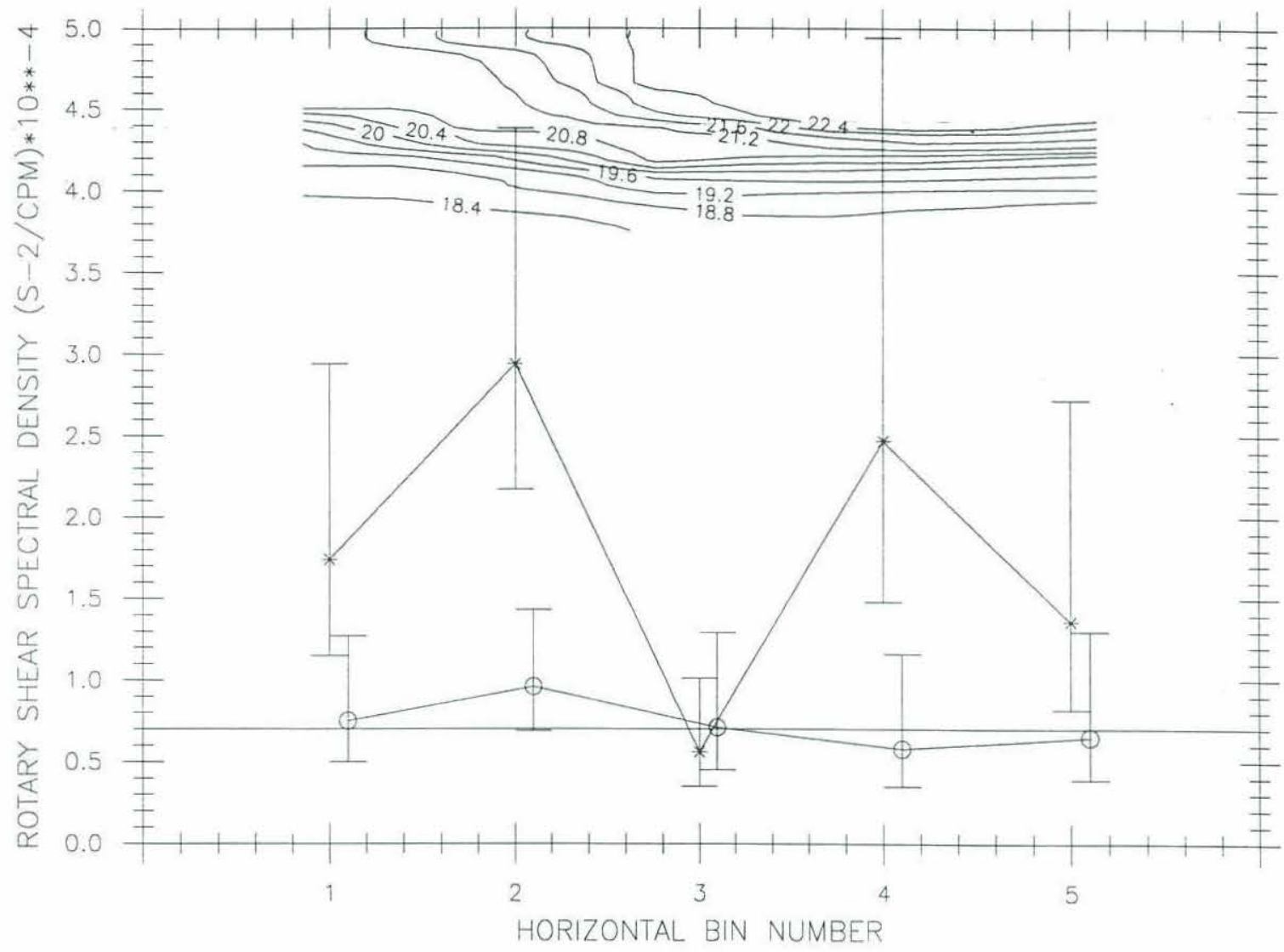

CW CCW COMPONENTS 125-250M

Fig. 2.7: WKB-scaled rotary shear spectral density, .0078 to .031 cpm, vs across-front bin, with $95 \%$ confidence intervals for the depth range $125-250 \mathrm{~m}$. The horizontal line at $.7 \times 10^{-4} \mathrm{~s}^{-2} / \mathrm{cpm}$ is the one component GM prediction for the average shear spectral density. 


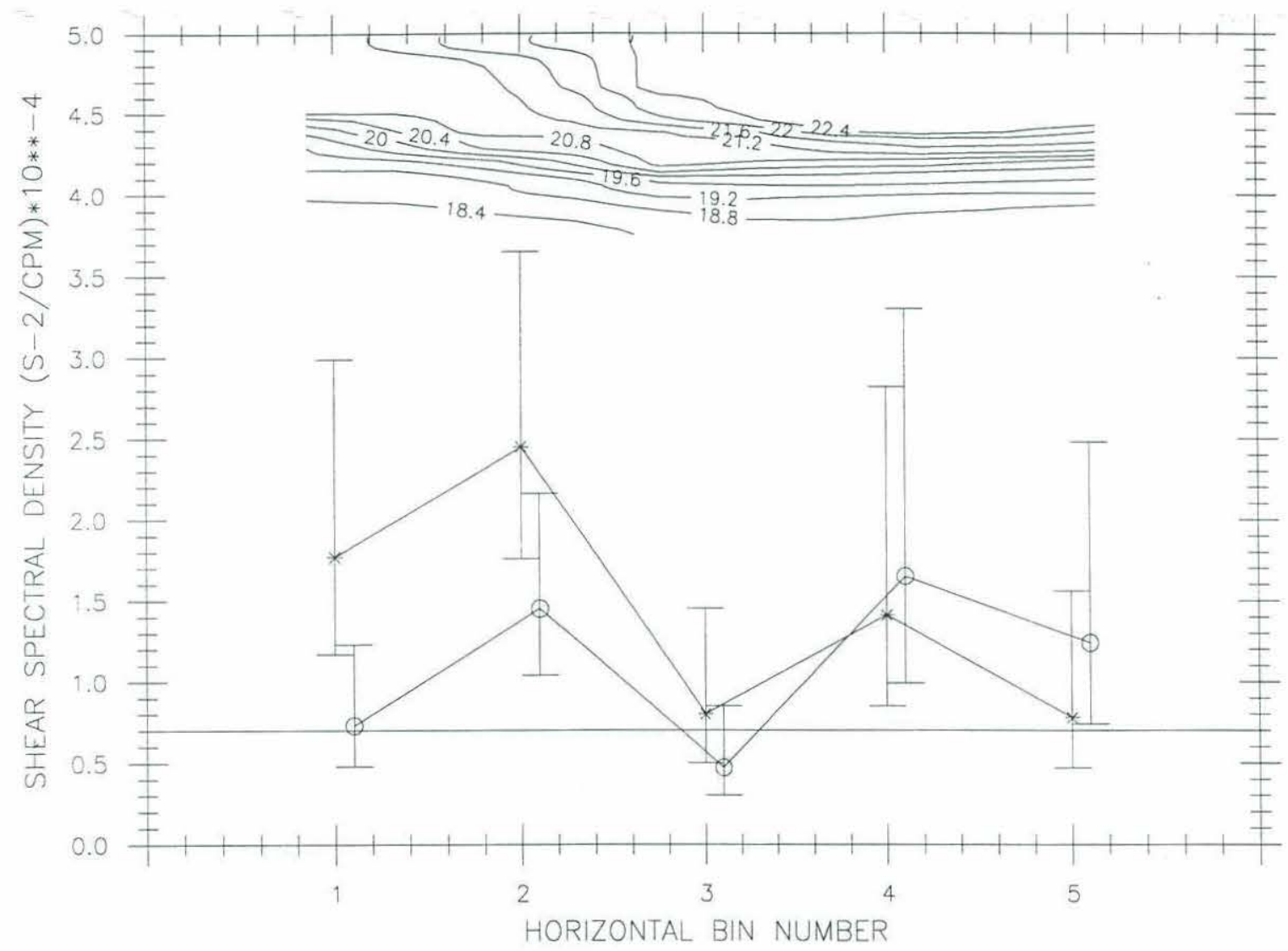

ACROSS ALONG COMPONENTS 125-250M

Fig. 2.8: Across- (star) and along-front (circle) WKB internal wave shear spectral density for .0078 to $.033 \mathrm{cpm}$ for the depth interval $125-250 \mathrm{~m}$, with $95 \%$ confidence intervals. 
front shear spectra (Figure 2.9) for bins 1-3 and the corresponding rotary shear spectra in Figure 2.6 revealed that the enhancement of across-front over along-front internal wave shear occurred in the same wavenumber band as the enhancement of $\mathrm{CW}$ over $\mathrm{CCW}$ phase rotation. Anisotropy of the near-inertial band-passed currents was also noted in the current meter data from the moored array (Weller et al., 1991)

In each of the first three bins, the across-front shear spectral density in the wavenumber band $0.0078-0.031 \mathrm{cpm}$ exceeded the along-front shear spectral density by a factor of approximately two (Figure 2.8). Considered individually, this factor of two is not statistically significant to the $95 \%$ confidence interval (roughly between the 80 and $90 \%$ c.i.). However, the averaging of all profiles in these three bins increases the degrees of freedom and produces a statistically significant result at the $95 \%$ confidence interval. As a consequence of the consistent nature of this pattern, we view this anisotropy in shear spectral density to be a robust feature of the internal wave field in the region of large background shears associated with the front.

Viewed individually, the majority of profiles in the frontal region revealed enhanced levels of across-front shear variance as compared to along-front shear. Most extreme are profiles 27 and 36 (Figure 2.10) which, when rotated to across/along-front coordinates, reveal very striking packets of near-monochromatic waves solely in the across-front direction. Both of these profiles were taken just on the warm side of the front in regions of large horizontal curvature of the background flow (both positive and negative) A hint of another 


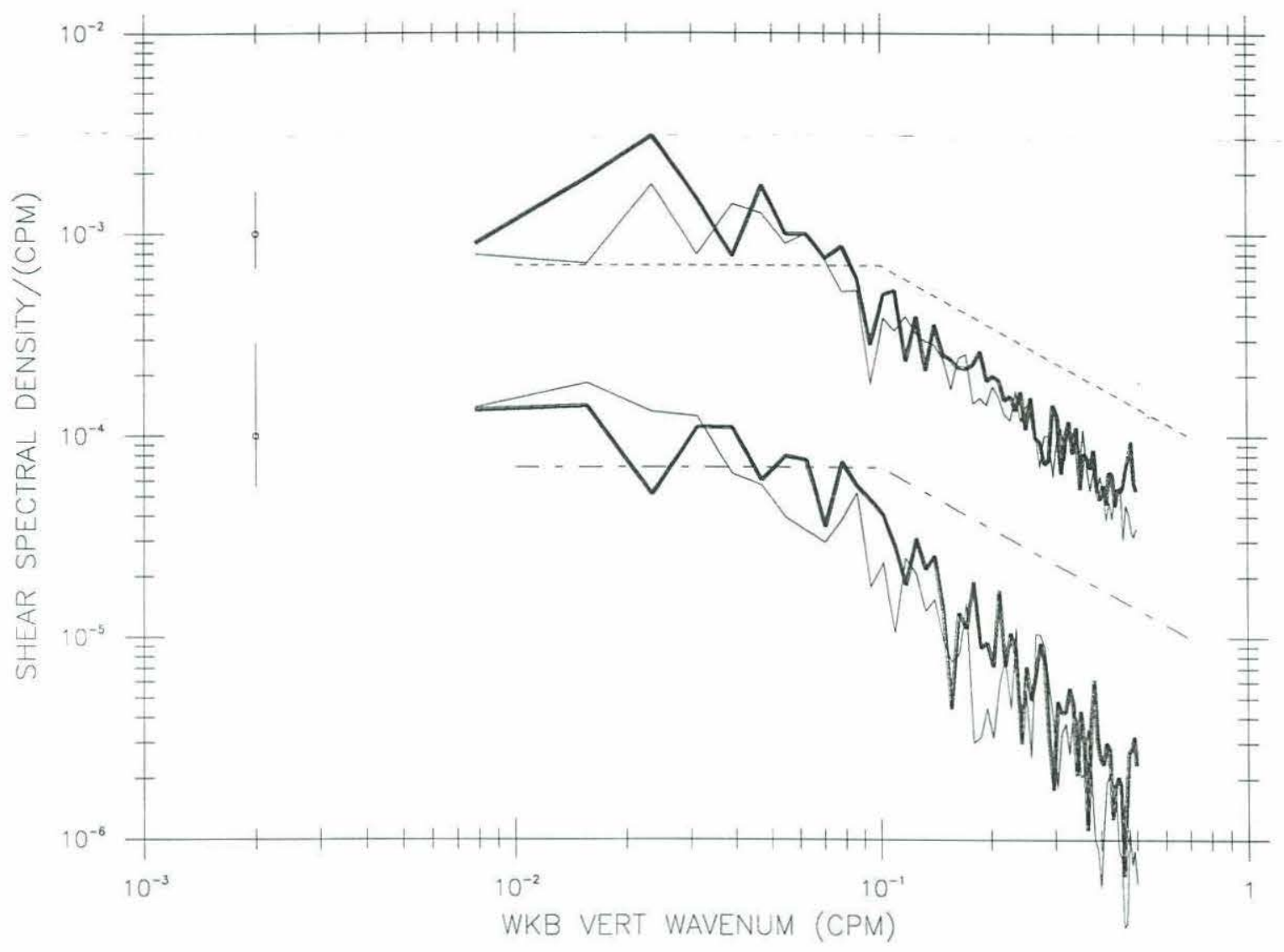

WKB $\vee U$

Fig. 2.9: Across- (thick) and along-front (thin) WKB shear spectra for the first three(upper pair) and last two (lower pair) horizontal bins in the depth interval $125-250 \mathrm{db}$. The upper set of spectra have been offset by one decade. Ninety-five percent confindence intervals are displayed to the left. 


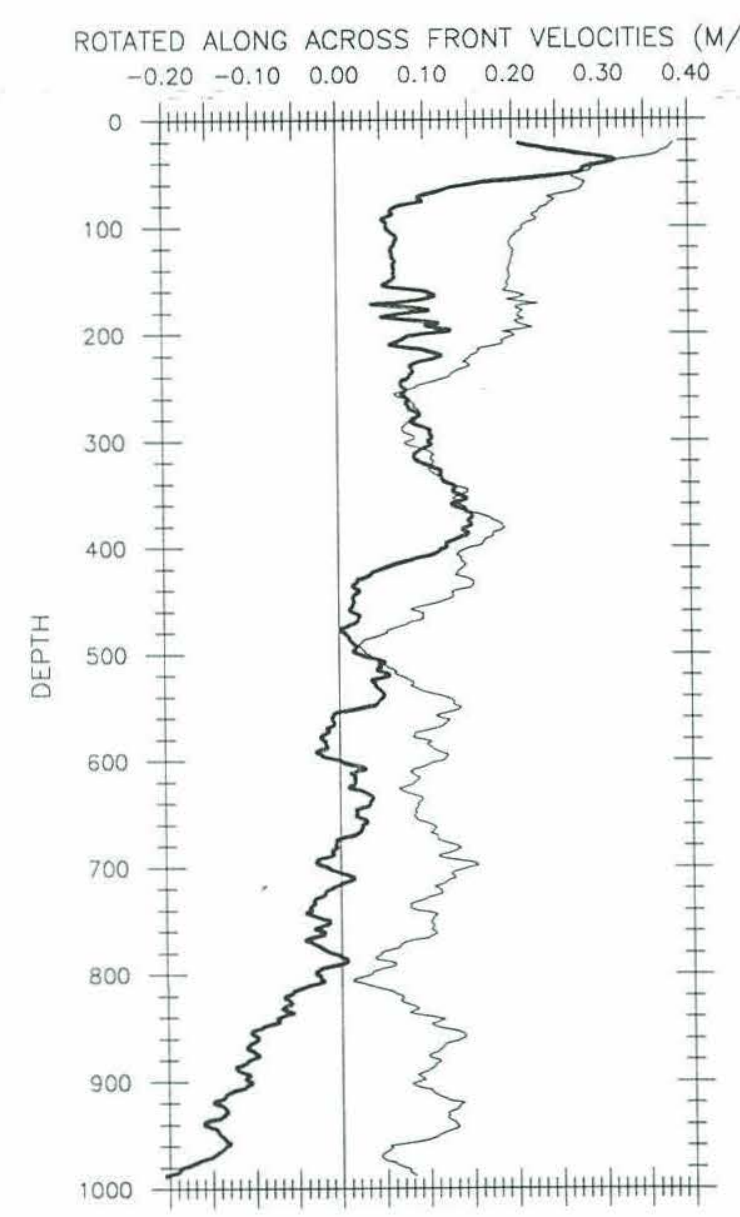

HRP 27
ROTATED ALONG ACROSS FRONT VELOCITIES (M/S $\begin{array}{lllllll}-0.20 & -0.10 & 0.00 & 0.10 & 0.20 & 0.30 & 0.40\end{array}$

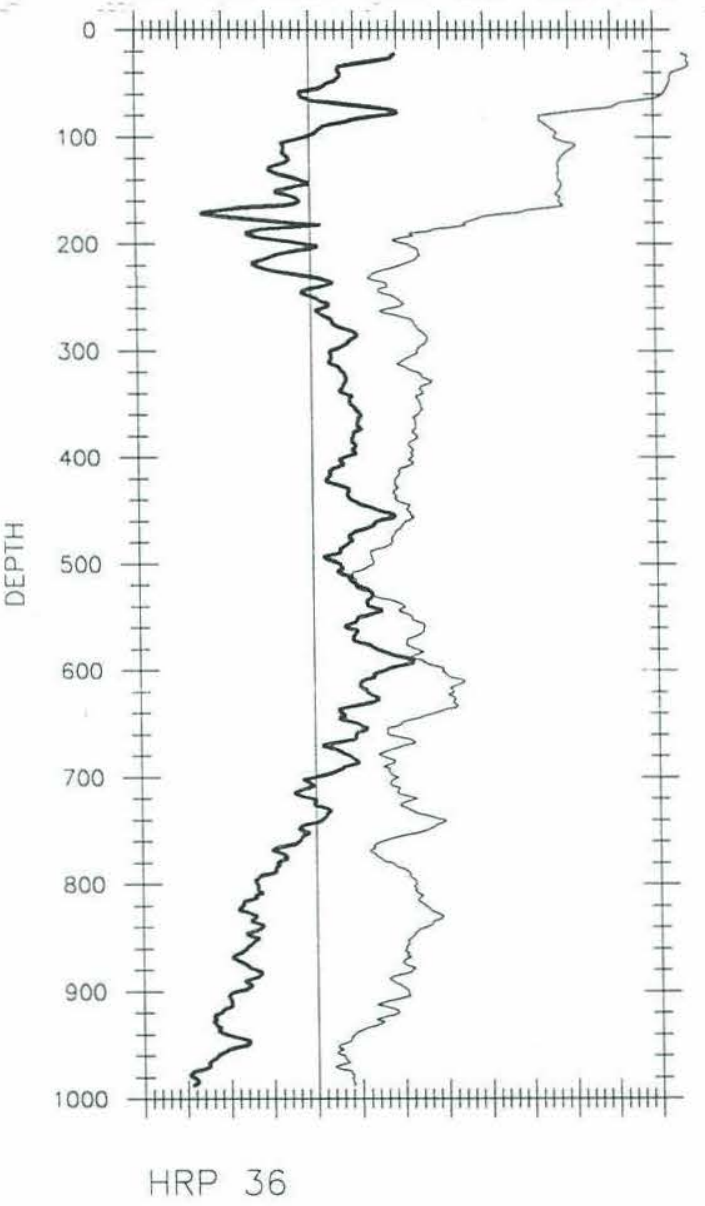

Fig. 2.10: Along- (thin) and across-front (thick) velocities for profiles 27(a) and 36(b). These profiles were taken immediately to the south of the surface front (bin 2, see Figures 2.1 and 2.2) Note the presence of large amplitude, monochromatic structures only in the across-front direction at a depth of about $200 \mathrm{~m}$. While a difference in across-front vs along-front internal wave shear was noted in bins 1,2 and 3 (Figure 2.8), such structures were noted only in bin 2 . 
such feature can also be found in profiles 34 and 35 of even smaller scale. No such features were found in the other horizontal bins. The degree of anisotropy in $S_{z}$ for bin 2 was not significantly different from that in bin 1 or 3 .

Statistically significant differences (95\% confidence interval) between the $\mathrm{CW}$ and $\mathrm{CCW}$ shear were intermittently apparent amongst the horizontal bins in the $18^{\circ} \mathrm{C}$ water and in the main thermocline, while in all cases the $\mathrm{CW}$ component was larger than the CCW (Table 2.1). No significant anisotropy or strong patterns of anisotropy were apparent in these depth ranges. A horizontal average produces the result that the $\mathrm{CW}$ component is significantly larger than the $\mathrm{CCW}$ component $(95 \%)$ in both the $18^{\circ} \mathrm{C}$ water and the main thermocline. (Table 2.1)

c) Time variability

An additional question to be addressed is the variability of the small-scale internal wave shear with respect to time: from previous work (Rubenstein and Roberts, 1986 and Wang, 1991), variation of the near-inertial internal wave energy level due to unsteady wind forcing was anticipated. Several wind events were observed during Phase II of FASINEX, with the largest of these preceding profile 26 (Weller et al., 1991). Simply averaging the 125-250 db shear statistic for profiles 26-36 and comparing this to the average of the preceding profiles produced the result that the total shear for the later profiles was statistically greater than those taken earlier. However, in as much as the sampling scheme is biased in favor of bin 2 for profiles 26-36, some horizontal variability may be aliased into 
this result. Hence it seems reasonable to weight each bin equally when producing the time averaged shear statistic. This procedure produced the result that the ratio of total shear for profiles 26-36 vs the previous profiles was 1.23 . This ratio was considerably below the $95 \%$ level of statistical significance, even when the number of degrees of freedom was based upon the total number of profiles analyzed. The weighting procedure will result in a lower number of equivalent degrees of freedom. Hence we conclude that the temporal increase in internal wave shear was not significant.

\subsection{Mixing Intensities}

This section examines the horizontal variability of the microstructure fields. Mixing events are thought to be directly linked to the occurrence of shear instabilities over scales of several meters, where the presence of supercritical Richardson number $(\mathrm{Ri}=$ $\left.\mathrm{N}^{2} / \mathrm{S}^{2} \leq 1 / 4\right)$ results in the growth of small scale unstable waves which eventually break, resulting in turbulent mixing (e.g. Thorpe, 1973b; Munk, 1981; Desaubies and Smith, 1982; see also Chapter

Four). Apart from boundary layers, gravity currents or the equatorial undercurrents, the vast majority of the shear in the ocean is associated with the internal wave field, not in the background flow. Richardson numbers calculated from the low order polynomial velocity fits and the background stratification defined in Section 2.3 are nearly always larger than 10 within the frontal region. The exception is at the base of the thermostad where Richardson numbers as low as 3 are found over a vertical extent of 
approximately 10 meters. Thus the shear in the background velocity structure of the front is not large enough by itself to result in mixing. The occurrence of supercritical Richardson numbers events is due to the variability in the the internal wave field. This is a stochastic process in which even rather modest shear variances (e.g. $<S^{2}>/ N^{2}=.5$ ) result in a finite probability of occurrence of supercritical Richardson number events, and the probability of occurrence increases dramatically over smaller scales where the shear variance is even larger (Chapter Four). The role of the background velocity structure of the front in producing heightened mixing is through the interaction of internal waves with the background to produce regions of elevated internal wave energy levels, (Kunze, 1985) which are in turn associated with heightened levels of turbulent dissipation (Gregg, 1989; Chapter Three). It is therefore expected that the heightened internal wave shear levels noted in Section 2.5 will result in increased mixing in the vicinity of the front.

Gregg (1989) has previously attempted to quantify turbulent dissipation rates as a simple function of the internal wave energy level and buoyancy frequency. That dissipation scaling was based upon dynamical models (Henyey et al., 1986; McComas and Muller, 1981) using the GM spectrum to calculate the average upwavenumber energy flux resulting from wave/wave interactions. These models equate the rate of turbulent dissipation with the average upwavenumber flux of energy in the internal wave dominated, low wavenumber $(.01-.1 \mathrm{cpm})$ portion of spectrum. We anticipate that the non-GM conditions (e.g. the presence of a back- 
ground flow, horizontal inhomogeneity, vertical anisotropy) noted in Section 2.5 may alter the strength of the wave/wave interactions and therefore affect the mixing rates. We apply the model of Gregg (1989) to ascertain whether deviations of the internal wave characteristics associated with an upper ocean front quantitatively affect the mixing rates.

The variability in the mixing intensity is addressed by estimating eddy diffusivities from both the Cox-Osborn model, $\mathrm{K}_{\mathrm{T}}=\chi / 2 .<\theta_{\mathrm{z}}>^{2}$ (Osborn and Cox, 1972), and the method of Osborn, $\mathrm{K}_{\rho}=.25 \varepsilon / \mathrm{N}^{2}$ (Osborn, 1980). The rate of thermal dissipation, $\chi$, is estimated from the micro-scale temperature gradients, and the rate of turbulent kinetic energy dissipation, $\varepsilon$, from air-foil velocity probes. Isotropy and a flux Richardson number of $R_{f}=.20$ have been assumed. (See Appendix One for details.)

During FASINEX, only three of the 23 HRP deployments which form the basis of the previous analysis were made with the shear probes. Estimates of $\chi$ are available for all HRP deployments. In addition, 15 EPSONDE (Oakey, 1988) stations were taken within an hour and a kilometer of the corresponding HRP deployments. In general, four EPSONDE profiles to depths of 225-250 m were obtained at an individual station. Both $\varepsilon$ and $\chi$ estimates are available for the EPSONDE profiles. These data are used to augment the statistical reliability of the diffusivity estimates.

It is implicitly assumed that the internal wave field is correlated over these space and time scales. Individual supercritical Richardson number events evolve over a time scale of approximately $2 \pi / \mathrm{N}$ (Chapter Four) and will not be temporally correlated between 
measurements. However, given the dominant near-inertial frequency content of the oceanic internal wave field, the internal wave field should be statistically stationary in the time between HRP and EPSONDE profiles. Near-inertial internal waves also have a low aspect ratio. As determined from the ratio between vertical to horizontal wavelengths, $\mathrm{k}_{\mathrm{h}} / \mathrm{m} \sim \mathrm{f} / \mathrm{N} \sim 1 / 100$. Near-inertial internal waves with vertical wavelengths greater than 25 meters should be coherent over the distance separating the measurements, implying the average shear level content over these vertical scales should be constant. However, waves with vertical scales smaller than 10 meters which are directly related to mixing events (Chapter Four) will not necessarily be coherent and thus some variability between EPSONDE and HRP microstructure estimates is to be expected. The rate of turbulent dissipation can be equated with the average upwavenumber flux of energy through the vertical wave number spectrum over vertical scales of tens to hundreds of meters (e.g. Henyey et al., 1986; McComas and Muller, 1981). Since the internal wave field with these vertical scales is coherent over the spatial and temporal differences separating the HRP and EPSONDE measurements, it is expected that sufficient averaging should produce similar diffusivity estimates. When averaged over the 125$250 \mathrm{db}$ window, coincident EPSONDE $\mathrm{K}_{\rho}$ and $\mathrm{K}_{\mathrm{T}}$ exhibited up to an order of magnitude scatter, similar to that found by Gregg et al., 1986. The HRP pressure averaged estimate was generally bounded by the upper and lower EPSONDE estimates for the corresponding HRP profile. The exception to this was when very low diffusivity estimates were obtained with the HRP profiles. While this difference 
is somewhat unsettling, it has not been attributed to any specific cause. Cruise averaged $\mathrm{K}_{\mathrm{T}}$ estimates for the 15 collocated HRP and EPSONDE deployments differ by only $40 \%$. Differences in processing and instrumentation are delineated in Appendix One.

The EPSONDE and HRP diffusivity estimates averaged over the 125-250 db window are presented in Figure 2.11 as a function of the horizontal bins defined in Section 2.3. Within each bin a scatter of an order of magnitude is apparent. Also apparent is an increase in diffusivity in bin 2 , the region of negative relative vorticity of the background velocity structure. The mixing $5-10 \mathrm{~km}$ to the south of the front in the region of positive relative vorticity (bin 3) is small, as are the diffusivity estimates for regions even further removed from the front to the south. Diffusivity estimates to the north are somewhat larger than those in the far field to the south. The pattern is identical to the variability in the internal wave field described in Section 2.5 (Figure 2.7).

Comparison of the diffusivity estimates to the internal wave field is facilitated by the dissipation parameterization of Gregg (1989) (see Chapter Three for a more thorough description). Gregg suggests:

$$
\begin{aligned}
& \varepsilon=7 \times 10^{-10}<\mathrm{N}^{2} / \mathrm{N}_{0}^{2}><\mathrm{S}_{10}^{4} / 2<\mathrm{S}_{10 \mathrm{GM}}^{2}>^{2}>\mathrm{W} / \mathrm{kg}, \text { or } \\
& \mathrm{K}_{\rho}=.25 \varepsilon / \mathrm{N}^{2}=6.1 \times 10^{-6} \mathrm{~m}^{2} / \mathrm{s}\left(\mathrm{S}_{10}^{4} / \mathrm{N}^{4}\right)
\end{aligned}
$$

where $\left\langle\mathrm{S}_{10}^{4}>\right.$ is 4.45 times the fourth moment of the shear based upon 10 meter first-differenced velocity profiles and $\left\langle\mathrm{S}_{\mathrm{GM}}^{2}\right\rangle=.72 \mathrm{~N}^{2}$. For the HRP profiles, the background stratification was defined on the basis of the $25 \mathrm{~m}$ sliding least squares fit described in Section 2.3. The estimates of the fourth moment of the ten meter shear were 


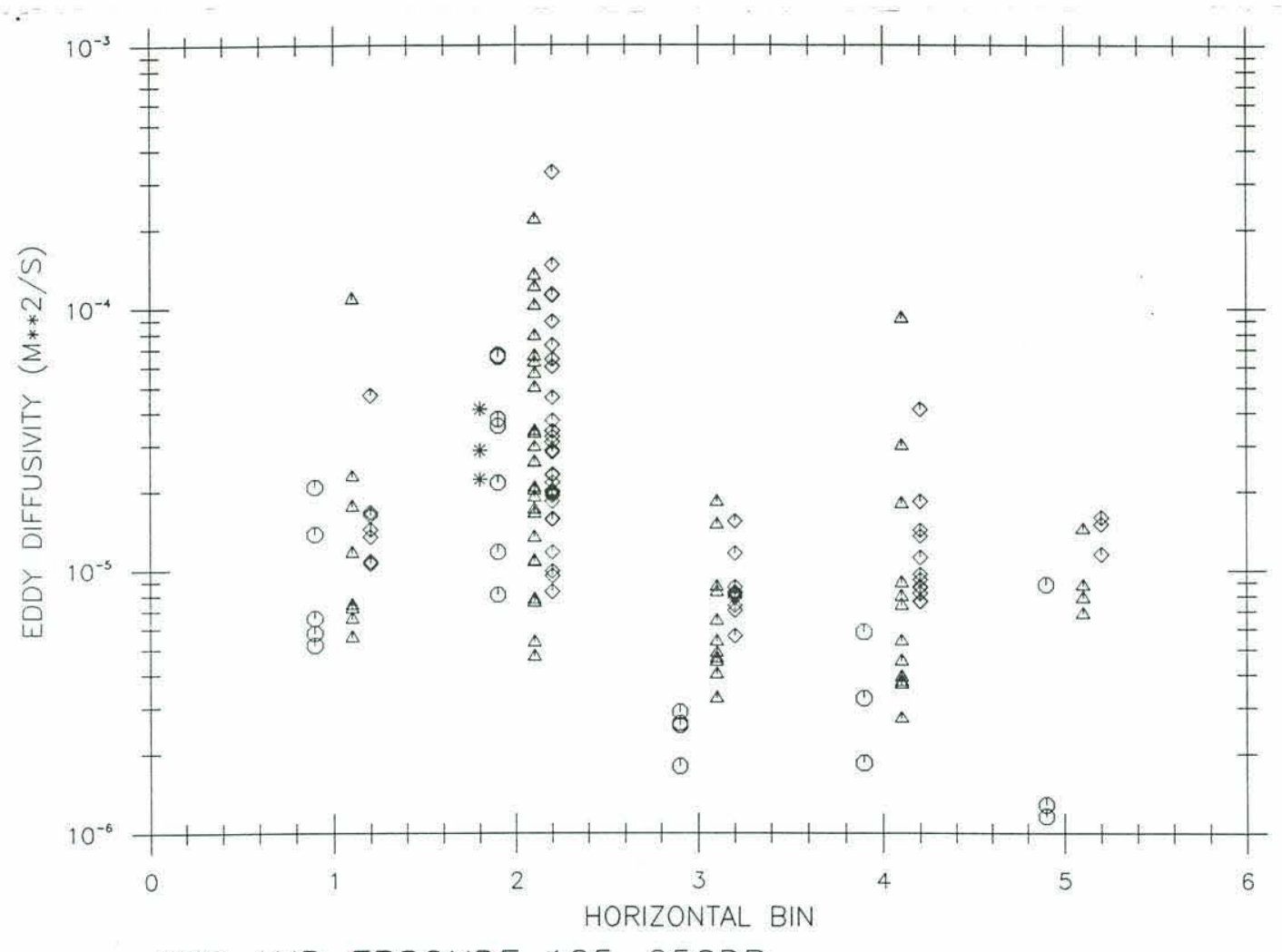

HRP AND EPSONDE 125-250DB

Fig. 2.11: Estimates of eddy diffusivity averaged over $125-250 \mathrm{~m}$. for individual casts and plotted with respect to their position relative to the front. The symbol o denotes HRP $\mathrm{K}_{\mathrm{T}}$, * HRP $\mathrm{K}_{\rho}, \Delta \mathrm{EPSONDE} \mathrm{K}_{\mathrm{T}}$ and $\triangle$ EPSONDE $K_{\rho}$ estimates. The various symbols are offset within each bin for clarity. 
divided by the local background stratification, averaged over the 125-250 db window, and then averaged for each bin. The diffusivity estimates for each HRP and EPSONDE profile were averaged by simply summing the estimates which appear in Figure 2.11. The resulting diffusivity and model estimates appear together in Figure 2.12. The Gregg (1989) model underestimates the observed diffusivity by a factor of 1.75 on average. This agreement is within the factor of two claimed by Gregg (1989) for that model.

The agreement between model estimates and observed diffusivity suggests that the overall upwavenumber flux of energy through the internal wave vertical wavenumber spectrum is insensitive to the presence of a background flow, anisotropy and inhomogeneity. In Chapter Three it was found that the frequency content of the internal wave field was important in determining the overall dissipation rate. In that study the frequency content was defined in terms of the ratio between the shear and strain variances, where the strain is the vertical derivative of the vertical displacement of an isopycnal relative to a background density profile defined by multiple profiles. For this data set the calculation of the strain is problematic. Within the thermostad, the background structure is sufficiently variable that significant errors are incurred if multiple profiles are averaged and the density profile smoothed. An alternative definition of $\eta_{\mathrm{z}}=\left(\left\langle\mathrm{N}^{2}\right\rangle-\mathrm{N}^{2}\right) /\left\langle\mathrm{N}^{2}\right\rangle$ is only true if the curvature of the density profile is small (Appendix Two) and cannot be applied to the thermostad region. It may be possible that the effects of anisotropy, inhomogeneity and the presence of the background velocity structure are important but are offset, in part 


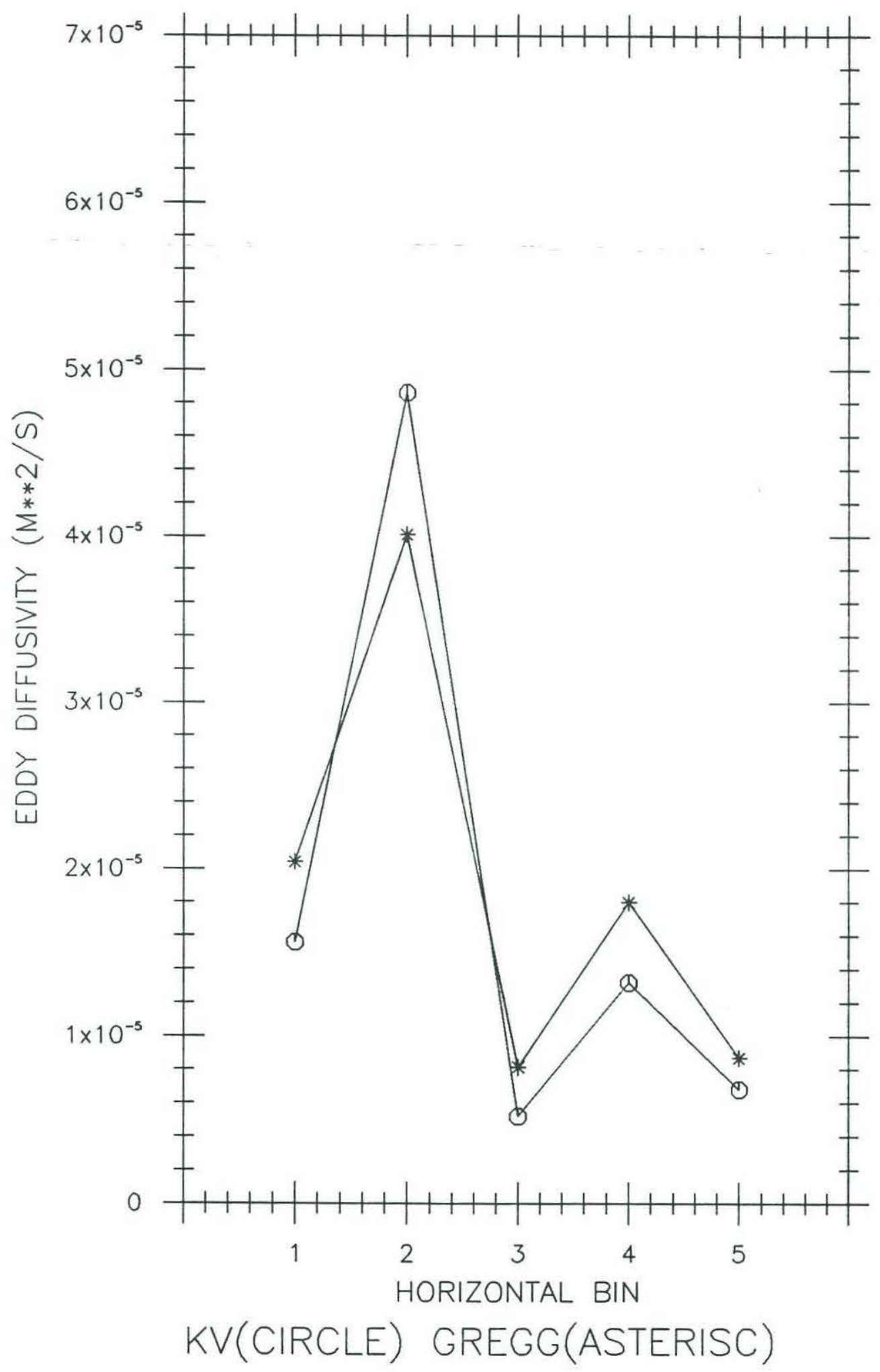

Fig. 2.12: Bin averaged estimates of eddy diffusivity, (o), from Figure 2.11 and the corresponding diffusivity prediction of Gregg (1989) (*). 
by differences in frequency content, or amongst themselves, in determining the average upwavenumber energy flux in the internal wave spectrum. The tendency for the model of Gregg to underestimate the observed diffusivities is inconsistent with the assumption of near-inertial frequency content, which leads to lower than anticipated dissipation rates on the basis of a simple energy level, buoyancy frequency scaling (Chapter Three).

\subsection{Discussion}

A subset of the 38 profiles of velocity, temperature and conductivity taken across an upper ocean front were found to exhibit a well defined front as characterized by Figure 2.2. The profiles were binned according to their location relative to the velocity structure of the front. The variability in the fine-scale characteristics was examined with the statistic, 'WKB shear spectral density between .0078 and .031 stretched cpm.'

The following points summarize our observations of the internal wave variability in the seasonal thermocline at the FASINEX front: 1) The CW estimates were significantly larger (95\% confidence intervals) than GM values in all but a region of positive relative vorticity associated with the presence of a subducted pycnostad. Conversely, CCW shear variance did not vary across the front and was not different from GM values. 2) The $\mathrm{CW}$ estimates were significantly larger than their CCW counterparts on the warm (negative relative vorticity) side of the front, exclusive of a region of positive relative vorticity associated with the subducted pycnostad. 
3) $\mathrm{CW}$ and total shear estimates $(\mathrm{CW}+\mathrm{CCW})$ within a region of positive relative vorticity related to a subducted pycnostad were significantly smaller than estimates in other regions of the front. 4) Significant anisotropy of the internal wave field was observed in the regions of large background shear (bins 1,2 and 3) with across-front shear variance exceeding along-front on scales of 128 to 32 stretched meters. 5) Both $\mathrm{CW}$ and total shear were largest just on the warm side of the surface velocity maximum (bin 2). However, the differences between shear levels in bins $1,2,4$, and 5 are not statistically significant (95\% confidence levels). 6) Despite the passage of several moderate intensity wind events during the experiment, internal wave shear variance in the later part of the cruise was not statistically different from the earlier part. This result may reflect the nonresonant character of the windstress with respect to the internal wave field.

Within the eighteen degree water and main thermocline regions, little horizontal variability was apparent. Overall, CW shear variance exceeded $\mathrm{CCW}$ at the $95 \%$ confidence levels in both depth intervals with total shear in the main thermocline slightly exceeding that of the $18^{\circ} \mathrm{C}$ water after WKB scaling.

The observed diffusivities agreed with the parameterization of Gregg (1989) despite the presence of a background flow and the obvious anisotropy and inhomogeneity within the wave field. Variability in the observed diffusivities was tracked by corresponding variability in the energy level parameterization. This agreement suggests that these deviations from the GM spectrum are unimportant in determining the average upwavenumber energy flux 
through the internal wave spectrum. The model of Gregg (1989) exhibited a tendency to underestimate the observed diffusivities (on average by a factor of 1.75). This tendency is somewhat puzzling in view of the frequency content scaling examined in Chapter Three, as it implies a higher than GM frequency content. This is inconsistent with a near-inertial wave/mean flow interaction scenario. It may be possible that the effects of anisotropy inhomogeneity and the presence of a background velocity field are important but are offset, in part by differences in frequency content, or amongst themselves, in determining the average upwavenumber energy flux in the internal wave spectrum. Unfortunately, estimation of the frequency content for these data is problematic.

Coincident with the increased small-scale internal wave shear, a signal of enhanced mixing was found in the region of negative relative vorticity associated with the outcropping of isopycnals and the pycnostad below. An estimate of the time scale for irreversible processes to mix the pycnostad is $\mathrm{T} \sim \mathrm{H}^{2} / \mathrm{K}_{\rho}$, where $\mathrm{H}$ is half the vertical extent of the pycnostad. For pycnostad heights of 40-80 meters (Figures 2.2 and 2.3) and an upper bound on $K_{\rho}$ of $1.0 \times 10^{-4}$ $\mathrm{m}^{-2} / \mathrm{s}$, the time scale is approximately 50 to 200 days. This is much larger than the 3 day time scale at which the frontal structure was observed to evolve (Pollard and Regier, 1992). Pollard and Regier also describe the presence of isolated plumes of subducted water of 50 meter vertical extent in the seasonal thermocline to the south of the FASINEX front. Since the mixing is smaller behind the front, such plumes will have correspondingly larger residence times of a year or greater. Homogenization of such features into the ambient 
stratification by irreversible processes over shorter time scales is possible if the plumes are strained by the mesoscale eddy field and their vertical extent reduced.

In a statistical sense, the present HRP shear estimates are much like the estimates of Kunze and Sanford (1984). They found little variation in their $\mathrm{CCW}$ energy statistic, a horizontally averaged factor of two increase in CW over CCW energy $\left(2.7: 1.3 \mathrm{~J} / \mathrm{m}^{3}\right)$, and a peak in $\mathrm{CW}$ energy 5 times the average CCW energy. For this study, the same results hold for the $\mathrm{CCW}$ shear statistic, the $\mathrm{CW}$ shear was an average of 2.6 times the $\mathrm{CCW}$ shear, and a peak value in the $\mathrm{CW}$ shear of 7.0 times the average CCW shear was noted. However, in contrast to Kunze and Sanford, large amplitude, horizontally coherent near-inertial internal waves were conspicuously absent from the data set, and a simple signal of near-inertial internal wave energy trapped to the warm side of the front was not observed. This is consistent with the very localized near-inertial signature seen in the seasonal thermocline by the instruments at the moored array (Weller et al., 1991). Wang (1991) addressed the problem of internal wave generation via inertial pumping due to the dispersion of inertial waves across a simple baroclinic upper ocean front. The density field was modeled after the front that Kunze and Sanford (1984) examined. A factor of four increase in model internal wave energy levels in the region of negative relative vorticity was noted in comparison to internal wave energy levels in the far field. In agreement to the model results, the largest enhancement of $\mathrm{CW}$ shear was found in bin 2, a region of negative relative vorticity associated with the surface expression of the front. Generally heightened CW 
shear levels were found for these data, exclusive of bin 3. Bin 3 was a region of positive relative vorticity and represented a physical situation not addressed in the Wang (1991) simulation.

Kunze and Sanford describe the presence of two wave groups distinguishable in their XCP profiles and noted that adjacent profiles were coherent. Such a signal is generally absent from this data set. Some of this may be due to the larger station spacing $(\mathrm{O}(10 \mathrm{~km})$ in comparison to the 2-4 $\mathrm{km}$ spacing in the case of Kunze and Sanford). However, there is general lack of distinct wave structures with a $\pi / 2$ phase shift between the velocity components which is indicative of a dominant near-inertial structure within the frontal region between 125-250 meters depth. In comparison, our profiles appear to be much more stochastic in nature. One of the wave packets found by Kunze and Sanford had a vertical wavelength $(2 \pi / \mathrm{m})$ of approximately 100 meters and an amplitude (u) of $.2 \mathrm{~m} / \mathrm{s}$, implying a Richardson number of $\mathrm{Ri}=\mathrm{N}^{2} / \mathrm{m}^{2} \mathrm{u}^{2} \sim .25$. In comparison, for the profiles in bin 2 , the vertical wavenumber shear spectrum needs to be integrated to wavelengths of less than 10 meters in order to obtain a shear variance of $4 \mathrm{~N}^{2}$. The measurements of Kunze and Sanford imply extremely enhanced levels of turbulent mixing in comparison to the mixing intensities found for this data set.

The problem of internal wave propagation in the subtropical front is not a simple one, and it is suggested that the lack of large scale internal wave structures as observed by Kunze and Sanford (1984), and Kunze (1985) may be due to the complex structure of the FASINEX front. Although we lack estimates of absolute velocity, the reversal of background shear within the subducted pycnostad 
represents a convoluted vorticity field and a further decrease of scales from that indicated by simple scaling arguments. Kunze (personal communication 1990) reports that the frontal structure he analyzed was not so convoluted. The region of negative relative vorticity (bin 2) was surrounded by regions of positive relative vorticity (bins 1 and 3 ). For near-inertial internal waves propagating into the front, these regions represent possible barriers (Kunze, 1985) and thus the incoming internal waves will be reflected. Kunze and Sanford suggest that the 100 meter vertical wavelength nearinertial internal wave they observed may have propagated into the frontal region and reflected off the positive relative vorticity ridge on the cold side of the front they analyzed. The spatial scale of the region of negative relative vorticity in the FASINEX front, $5-7 \mathrm{~km}$ (Figure 2.4) was 2 to 3 times smaller than the corresponding scale of the front in which Kunze and Sanford obtained their XCP profiles from. For a given vertical wavelength, this difference in horizontal scales implies that internal waves which are generated via inertial pumping processes at the base of the mixed layer have higher frequencies. For the linear near-inertial internal wave dispersion relation in the absence of a background flow, $\left(\mathrm{k}_{\mathrm{H}} / \mathrm{m}=\left(\omega^{2}-\mathrm{f}^{2}\right)^{1 / 2} / \mathrm{N}\right)$, an increase in the aspect ratio of a factor of 2 to 3 implies an increase in $\omega^{2}-f^{2}$ of a factor of 4 to 9 , and such waves may be sufficiently super-inertial to escape the trapping effects of the background flow. This argument is heuristic. The production of internal waves via inertial pumping in a baroclinic front is a non-linear process (Wang, 1991) and the detailed character of the generated waves may depend intimately on the density structure of the particular front 
used in numerical simulations. In this experiment the scenario of near-inertial wave trapping in regions of negative relative vorticity of Kunze (1985) appears most clearly as the depressed level of internal wave shear in the third bin.

The small-scale internal wave shear showed evidence of anisotropy in the seasonal thermocline. To our knowledge, this is the first report of anisotropy in terms of vertical wavenumber in regions removed from topography. Anisotropy has been reported before in the case of frequency domain velocity spectra (Frankignoul 1976, Ruddick 1977). They observed anisotropies of $50 \%$ for frequencies much larger than inertial in regions of strong background flow. In contrast, the moored FASINEX array data revealed indications of anisotropy in the near-inertial band-passed currents (Weller et al., 1991).

A plethora of mechanisms have been suggested as possible causes of anisotropy. Doppler shifting of internal waves past the profiler (Frankignoul 1974), should not significantly affect these data as the fall rate of the profiler $(\sim 65 \mathrm{~cm} / \mathrm{s})$ was larger than the largest vertical or horizontal internal wave phase speeds in this analysis ( 3 $\mathrm{cph} / 2 \pi(1 / 128 \mathrm{~m}) \sim 11 \mathrm{~cm} / \mathrm{s})$. Critical layers would preferentially remove part of the along-front wave number spectrum. Ruddick (1980) calculated that critical layer absorption would produce an anisotropy of less than $20 \%$ for currents less than $60 \mathrm{~cm} / \mathrm{s}$ and concluded that both his and Frankignoul's observations of $50 \%$ anisotropies could not be accounted for by critical layer effects. This anisotropy factor decreased with decreasing Eulerian frequency and decreasing background flow velocities. Our observed factor of two 
difference between across- and along-front internal wave shears translates into an anisotropy of $33 \%$. This figure is not strictly comparable to Frankignoul and Ruddick's observations as we have effectively integrated over all internal wave frequencies with a single vertical profile and one would expect that an energy weighted integration of Ruddick's anisotropy factor through the frequency domain would result in much smaller expected values for the anisotropy. Both Frankignoul and Ruddick discuss anisotropy in the context of Muller's (1976) weakly nonlinear, wave/wave mean flow interaction scenario. Ruddick and Joyce (1979) and Frankignoul and Joyce (1979) went on to determine that the wave momentum fluxes were at least a factor of 20 times smaller than those proposed by Muller. It is therefore unlikely that Muller's scenario is a viable explanation for internal wave anisotropy.

In general, any internal wave generation mechanism which results in a greater tendency to across-front variability than alongfront variability will result in increased across-front versus alongfront internal wave velocities. Both geostrophic adjustment and inertial pumping can be formulated in terms of 2-D problems and thus potentially result in anisotropic wave fields. One might anticipate observing such fields if the variability of the background flow in the across-front direction was much larger than that in the along-front direction. However, we again caution that these simple expectations could be considerably altered by wave/mean flow interactions. Profiles 27 and 36, with their near-monochromatic features, suggest the possibility of some form of instability. The diffusive instability of a baroclinic vortex results in high 
wavenumber radially oriented variability (McIntyre, 1970). Weller et al. (1991) suggests that these features may be due to internal waves with high intrinsic frequencies approaching a critical layer in the horizontal. (High frequency waves have critical layers in the horizontal and turning points in the vertical while the opposite is true of near-inertial internal wave intrinsic frequencies (Eriksen, 1988). However, high frequency waves have a small shear to strain ratio (Kunze, Williams and Briscoe, 1990) and large strains did not appear to be obviously associated with the large shear features in profiles 27 and 36. Mied et al. (1990) suggest that the Doppler shifting of near-inertial waves may act to filter out all but crossstream propagating waves and thus result in an inhomogeneous wave field. Unfortunately, we lack the information on the complete horizontal derivatives of the background flow, horizontal wavenumbers, and time resolution necessary to evaluate these different mechanisms of generation, absorption, and interaction for creating wave anisotropy. We have attempted to interpret the anisotropy of the small-scale shear field in terms of internal wave dynamics. Researchers have found the small-scale shear field to be dominated by internal wave band frequencies in regions of small large-scale background flows (Kunze and Sanford, 1991, D'Asaro and Morehead, 1991). However, in the absence of any time domain information, the predominance of internal wave motions cannot be verified for these data.

Enhanced internal wave shear was found in the vicinity of the FASINEX front. This in turn was related to an enhancement of mixing within the region of negative relative vorticity. However, a 
simple signal of internal wave trapping is not observed. Possible reasons for this include the short length scales and the complex structure of the FASINEX front. The enhancement of across-front internal wave shear over along-front shear implies the possible existence of wave/mean flow interaction processes not addressed by Kunze (1985) which further complicate the problem of internal wave propagation. 
Chapter Three

Finescale Parameterizations of Turbulent Dissipation

\subsection{Introduction}

This study of dissipation parameterizations based upon models of the internal wave field is motivated by previous studies which indicate a wide disparity between estimates of vertical eddy diffusivities from abyssal regions and those from the main thermocline. Estimates of vertical eddy diffusivities based upon hydrographic data at abyssal stratifications (e.g. Hogg et al., 1982) are 'large', i.e. $\mathrm{O}\left(1-10 \mathrm{~cm}^{2} / \mathrm{s}\right)$. Models based upon various types of data which attempt to solve for an abyssal eddy diffusivity usually produce similar estimates (Wyrtki (1961), Munk (1966), Pickart et al. (1989), Joyce et al. (1986)). Direct estimates of dissipation in the thermocline give estimates of vertical eddy diffusivities at least an order of magnitude smaller, perhaps two, e.g. Gregg and Sanford (1988). Advective/Diffusive model studies of tracers and inversions of data from the thermocline in mid-gyre regimes produce diffusivities consistent with the direct estimates, e.g. Jenkins (1980), Olbers (1989).

Turbulence in the ocean is generally presumed to be driven by the vertical shear. Away from 'special' regions such as sills, overflows, or equatorial undercurrents, the vast majority of the vertical shear is in the internal wave field and not the background flow. Hence an explanation of the increase in eddy diffusivities at 
abyssal depths has been sought in terms of the modification of the internal wave field with depth. Several mechanisms have been invoked by previous investigators which might give large eddy diffusivities at depth:

1) An intrinsic buoyancy scaling of the turbulence levels which predicts $\varepsilon / \mathrm{N}^{2}$ is inversely proportional to $\mathrm{N}$ at constant (buoyancyscaled) internal wave shear levels

2) Wave-mean flow interaction

3) bottom reflection processes

4) bottom generation

5) enhanced effective vertical diffusivities due to "boundary mixing"

In considering the efficacy of 1-4 there appears to be at least two important parameters in the problem: a buoyancy scaled shear level, E, and the buoyancy frequency, N. The gist of the first hypothesis is that $\varepsilon / \mathrm{N}^{2}$ varies inversely with $\mathrm{N}$ at constant $\mathrm{E}$, while hypotheses 3 and 4 are concerned with the enhancement of $\mathrm{E}$ near the bottom, which is typically a region of low N. Internal waves reflecting from a sloping boundary tend to be scattered into small wavelengths (Eriksen, 1985), resulting in heightened shear levels in the vicinity of topographic features. Enhanced small-scale shear levels may also be noted if the internal waves generated by a background flow or barotropic tidal flow over topography (Bell, 1975) have characteristically small wavelengths. Hypothesis 2 (Kunze, 1985) may be important in regions such as deep western boundary currents. Deep western boundary currents present potential critical layers for both freely (downward) propagating and 
upward propagating, topographically generated lee waves. In hypothesis 5 (e.g. Garrett, 1990), the across slope flows associated with the near boundary enhancement of dissipations next to a sloping bottom potentially implies enhanced effective diffusivities.

In this study we concentrate on the first hypothesis. We do so in the context of comparing observations of turbulent dissipation to predictions from a number of dynamical and kinematical parameterizations of dissipation as a function of $\mathrm{E}, \mathrm{N}$ and frequency content. The present work has three important differences from earlier work: 1) a data set with greatly expanded parameter range in $\mathrm{N}, 2$ ) use of an internally consistent methodology for estimating $\mathrm{E}$ and 3) the consideration of frequency content as an independent variable. The parameter range in $\mathrm{N}$ is expanded by roughly a factor of two over the study of Gregg (1989), which allows us to robustly test between competing models. By avoiding the errors in the methodology of computing $\mathrm{E}$ we find that such errors may have masked important discrepancies between the models and the data in the past. These discrepancies lead us to formulate the model/data comparison in a format which explicitly includes the frequency dependence.

In a relatively isotropic, homogeneous subset of our data having little variation in $\mathrm{E}$ and frequency content, we find the scaling of Gargett and Holloway (1986), also Gargett (1990) (i.e. $\varepsilon \sim \mathrm{EN}^{3 / 2}$ ) and that of Munk (1981) (i.e. $\varepsilon \sim \mathrm{E}^{2} \mathrm{~N}^{3 / 2}$ ) to be inconsistent with an observed buoyancy scaling of approximately $\mathrm{N}^{2}$. We believe these results to be sufficient to exclude an intrinsic buoyancy scaling and 
suggest that the heightened abyssal diffusivities are due to either one or several of the remaining hypothesis acting in concert.

Two other models (Henyey et al. 1986, McComas and Muller 1981) both exhibit an approximate scaling of $\varepsilon \sim \mathrm{E}^{2} \mathrm{~N}^{2}$ and are consistent with the observed buoyancy scaling of the aforementioned data. These models equate $\varepsilon$ with the average flux of energy through the vertical wavenumber spectrum due to nonlinear wave/wave interactions. Such an approach is valid if the spectrum which forms the basis of the calculation is statistically stationary with respect to the nonlinear interactions. Results from both dynamical models indicate that the GM spectrum (Munk, 1981) is stationary. Data from a warm core ring (dominated by near inertial frequency motions) and region of steep topography (characterized by higher frequency motions) are analyzed in order to examine how deviations from the assumed GM spectrum may affect the observed dissipation rates. The only obvious trend in the diffusivities vs the model predictions based upon energy levels (E) and buoyancy frequency was in terms of frequency content. A frequency content scaling for the two dynamical models (Henyey, 1991) is discussed under the assumption that for small perturbations in frequency content, the scaling of the average energy fluxes at high vertical wavenumber for a spectrum which is not in equilibrium with respect to wave/wave interactions retains the scaling of individual waves propagating in an otherwise statistically stationary (nominally GM) field.

Chapter Three is organized as follows: the models are reviewed briefly in Section 3.2. In Section 3.3 the work of Gregg (1989) is examined in light of the criticisms of Gargett (1990). Details related 
to data analysis are discussed in Section 3.4. Section 3.5 consists of two parts. The first examines the issue of buoyancy scaling and the second addresses the dependence of turbulent dissipation upon the energy level and frequency content of the internal wave field. The results are reviewed and discussed in Section 3.6. Relevant descriptions of the instrument and further details of the data analysis may be found in Appendix One. A discussion of the GM spectrum and notation, and relevant linear internal wave parameters is relegated to Appendix Two. A summary of the notation used in Chapter Three appears in Table 3.1 below.

3.2 A Review of Models of Dissipation Due to Internal Waves

Before a detailed model/data comparison we attempt to briefly review th relevant models. Much of this information is contained in Muller et. al. (1986) and in Gregg (1989). We present this information for the following reasons:

1) Our implementation of the models differs in detail from that of Gregg (1989). We wish to delineate these differences.

2) We expand the model of Gargett and Holloway (1984) to include the suggestions of Gargett (1990).

3) We attempt to address the implications of a non-GM frequency spectrum for the models of Henyey et. al. and McComas and Muller (1981) in light of recent work by Henyey (1991). 
Table 3.1: Notation for the internal wave models and parameters of the GM spectrum.

\begin{tabular}{|c|c|}
\hline$\hat{\mathrm{E}}=\mathrm{E}_{\mathrm{GM}} \mathrm{b}^{2} \mathrm{~N}_{0} \mathrm{~N}$ & $\begin{array}{l}\text { dissipation rate of turb. kinetic energy } \\
\text { total energy }\end{array}$ \\
\hline $\mathrm{E}$ & dimensionless spectral level \\
\hline $\mathrm{E}_{\mathrm{GM}}=6.3 \times 10^{-5}$ & GM specification of $\mathrm{E}$ \\
\hline $\begin{array}{l}\mathrm{b}=1300 \mathrm{~m} \\
\mathrm{~N}\end{array}$ & $\begin{array}{l}\text { scale height of the thermocline } \\
\text { buovancy frequency }\end{array}$ \\
\hline $\mathrm{N}_{\mathrm{o}}=.00524 \mathrm{~s}^{-1}$ & reference frequency \\
\hline $\begin{array}{l}\mathrm{f}=2 \times 7.3 \times 10^{-5} \sin (\text { lat }) \\
\mathrm{m}\end{array}$ & $\begin{array}{l}\text { Coriolis parameter } \\
\text { vertical wavenumber }\end{array}$ \\
\hline $\begin{array}{l}\mathrm{m}_{*}=3 \pi \mathrm{N} / \mathrm{bN}_{\mathrm{o}} \\
\mathrm{k}_{\mathrm{h}}\end{array}$ & $\begin{array}{l}\text { parameter in the GM vertical spectrum } \\
\text { horizontal wavenumber }\end{array}$ \\
\hline$\omega$ & frequency \\
\hline $\mathbf{k}$ & wavenumber vector \\
\hline $\mathbf{x}$ & position vector \\
\hline
\end{tabular}

$\underline{\text { McComas and Muller (1981) }}$

McComas and Muller (1981), (also as MM) used results from weak resonant interaction theory to produce a dynamical balance for the internal wave field. In their model they utilized the dominance of the parametric subharmonic instability at low frequencies and large vertical wavenumbers to construct a statistically stationary solution in wavenumber space, i.e. $\mathrm{E}(\mathrm{k}, \mathrm{t})=\mathrm{E}(\mathrm{t}) \mathrm{E}(\mathrm{k})$. They then sought and obtained similar solutions for the high frequency $(4 \mathrm{f}<\omega<\mathrm{N})$ portion of the vertical wavenumber spectra by assuming that the non-linear transfers were dominated by the induced diffusion mechanism. For these spectral solutions, the energy fluxes of both mechanisms are toward low frequencies and high vertical wavenumber. The time scale and hence the energy flux for the high frequency portion of the spectra was determined by requiring the high and low frequency spectra to match at their common boundary $(\omega=4 \mathrm{f})$. 
The induced diffusion (id) mechanism can be characterized as the scattering of a high frequency, high wave number wave by a low frequency, low wavenumber wave. This process acts as a diffusion of wave action in wavenumber space and is most efficient in vertical wavenumber. This efficiency implies relatively small changes in horizontal wavenumber and thus the energy fluxes are towards lower frequencies.

The parametric subharmonic instability (psi) is characterized by the decay of a low wavenumber wave into two waves of half the frequency. This resonance condition requires, via a linear dispersion relation, that the two resulting waves have smaller vertical scales (by a factor of $\left.(1.5 f /(\omega-f))^{1 / 2}\right)$, where $\omega$ references the double frequency wave (MM).

One can produce an estimate for the rate of kinetic energy dissipation by matching the time scales for dissipation and the nonlinear internal wave fluxes (MM):

$$
\varepsilon=\hat{\mathrm{E}} / \tau_{\mathrm{diss}}=\hat{\mathrm{E}}\left(\tau_{\mathrm{psi}}+\tau_{\mathrm{id}}\right)^{-1}
$$

where $\tau_{\mathrm{psi}}$ and $\tau_{\mathrm{id}}$ are the time scales for the parametric subharmonic instability and induced diffusion mechanisms, respectively, and $\hat{E}$ is the total energy of the internal wave field. The energy flux under the induced diffusion mechanism is determined by the boundary conditions with the low frequency portion of the spectrum (dominated by the psi) and accounts for approximately $40 \%$ of the total energy flux (Muller et. al., 1986). Since the time scales for the two mechanisms have the same functional form, $\varepsilon=\hat{\mathrm{E}} / .6 \tau_{\mathrm{psi}}$. The time scale for the psi mechanism is given as (MM): 


$$
\tau_{\mathrm{psi}}^{-1}(\mathbf{k})=\frac{27}{16} \frac{\pi \mathrm{f}}{\mathrm{N}^{2}} \mathrm{~S}\left(\frac{\mathrm{m}}{\mathrm{x}}, 2 \omega\right)
$$

where $x=\sqrt{10}$ (the average value of $\left.(1.5 f /(\omega-f))^{1 / 2}\right)$ for a GM spectrum and $S(m, \omega)$ is a shear 'content' spectrum. Hence the time scale is proportional to the shear content of the double frequency wave with a vertical wave number, $\mathrm{m}$, $\mathrm{x}$ times smaller. For a content spectrum of the form $\mathrm{S}(\mathrm{m}, \omega)=\hat{\mathrm{Em}^{2}}\left(\mathrm{fm}_{*} / \omega \mathrm{m}\right)$, the time scale is uniform in $\mathrm{m}(\mathrm{MM})$. Evaluating $\tau$ at $\left(\mathrm{m}_{*}, \mathrm{f}\right)$ reduces eq. 3.1 to

$$
\varepsilon=\frac{1}{.6} \frac{27}{32} \frac{\pi \mathrm{f}}{\mathrm{xN}^{2}} \hat{\mathrm{ES}}_{*}=\frac{1}{.6} \frac{27}{32} \frac{\pi \mathrm{f}}{\mathrm{xN}^{2}} \mathrm{~m}_{*}^{2} \hat{\mathrm{E}}^{2}(\mathrm{~W} / \mathrm{kg}) .
$$

For values of $\hat{E}$ and $m_{*}$ from Munk (1981) (Table 3.1) this becomes $\varepsilon=2.1 \times 10^{+8} \mathrm{f} \mathrm{E}_{\mathrm{GM}}^{2} \mathrm{~N}^{2}$.

At 32.5 North, $\varepsilon\left(\mathrm{E}_{\mathrm{GM}}, \mathrm{N}_{\mathrm{o}}\right)=1.79 \times 10^{-9}(\mathrm{~W} / \mathrm{kg})$.

The frequency scaling is derived by considering the transport integral (Henyey, 1991). For the case of induced diffusion, the transport integral reduces to approximately:

$$
\mathrm{D}=\frac{\mathrm{k}_{\mathrm{h}}^{2}}{2} \int \mathrm{d}\left(\mathrm{S}^{2}\right) \delta\left(\Omega-\mathbf{v}_{\mathbf{g}} \cdot \mathbf{K}\right),
$$

where lower case variables correspond to a smaller vertical scale wave being sheared by a larger vertical scale (upper case) wave, $\mathbf{v g}$ is a group velocity and $d\left(S^{2}\right)$ is the shear variance at $\mathbf{K}$. The expression is simplified by noting that the vertical component dominates, the delta function scales inversely as its argument (which is taken to be $\left.v_{g z} M\right)$, and that $d\left(S^{2}\right)$ is $M S_{z}$, where $S_{z}$ is the shear spectral density. Hence the diffusivity reduces to $\mathrm{D} \sim \mathrm{S}_{\mathrm{z}} \omega \mathrm{m}^{3} / \mathrm{N}^{2}$ for hydrostatic waves. If a power law dependance is invoked for the vertical wavenumber spectrum, the expression for the dissipation becomes: 


$$
\varepsilon \sim \mathrm{D} \frac{\delta}{\delta \mathrm{m}}\left(\frac{\mathrm{d} \hat{\mathrm{E}}}{\mathrm{dm}}\right) \sim \mathrm{Dm}^{-1} \frac{\mathrm{d} \hat{\mathrm{E}}}{\mathrm{dm}}
$$

Since $S_{z}=m^{2} \frac{d \hat{E}}{d m}, \varepsilon \sim m^{4}\left(\frac{d \hat{E}}{d m}\right)^{2} \omega / N^{2}$. For the GM spectrum, $\frac{d \hat{E}}{d m} \sim \frac{N^{2} E}{m^{2}}$, so that

$$
\varepsilon \sim \mathrm{N}^{2} \mathrm{E}^{2} \omega .
$$

\section{Henyey, Wright and Flatte (1986)}

A major problem with the resonant interaction theories is the validity of the weak interaction assumption for high frequency and high wavenumber waves (Muller et. al. 1986). An alternative approach to the problem is that of Henyey et al., (1986) (HWF), who attempt to describe the internal wave interactions via an eikonal (ray-tracing) approach and calculate the energy fluxes with the help of MonteCarlo simulations of the ray equations. The ray equations are:

$$
\begin{aligned}
& \dot{\mathbf{x}}=\frac{\delta \sigma}{\delta \mathbf{k}}+\mathbf{u} \\
& \dot{\mathbf{k}}=-\frac{\delta \omega}{\delta \mathbf{x}}=-\frac{\delta \sigma}{\delta \mathbf{x}}-\frac{\delta}{\delta \mathbf{x}}(\mathbf{u} \cdot \mathbf{k})
\end{aligned}
$$

with the Eulerian frequency linking the two expressions,

$$
\omega=\sigma+\mathbf{u} \cdot \mathbf{k} .
$$

The intrinsic frequency, $\sigma$, is given by the linear internal wave dispersion relation:

$$
\sigma^{2}=\left(N^{2} k_{h}^{2}+f^{2} m^{2}\right) /\left(k_{h}^{2}+m^{2}\right) .
$$

Thus the velocity of a wave packet in physical space, $\mathbf{x}$, is the sum of a group velocity and the advection by the background flow. The evolution of the wavenumber, $\mathbf{k}$, is controlled by the WKB scaling of 
$\delta \sigma / \delta \mathbf{x}$ and interaction with the shear of the background flow through the Doppler shift, $\delta(\mathbf{u} \cdot \mathbf{k}) / \delta \mathbf{x}$.

A number of approximations are made in the simulations. The equations formally apply to the total velocity field. However, the ray tracing requires the assumption of a scale separation between the wave packet being traced and the background (Henyey and Pomphrey, 1983). HWF chose to use a small amplitude test wave and model the background flow $\mathbf{u}$ as a Gaussian process with a modified GM spectrum. The small amplitude assumption implies that the test waves (at small scales) do not interact with the background (defined as the large scale internal wave field). The background flow is filtered to exclude waves whose vertical wavelength is smaller than that of the test wave. The numerical simulations are carried out until the vertical wavelength of the test wave reaches $.2 \mathrm{cpm}$, where the wave is considered to have dissipated, presumably via shear instability. The modification to the GM spectra consisted of the addition of an extra $\mathrm{m}^{-1}$ dependance for vertical wavenumbers greater than .1 cpm. Although larger than GM energy levels were not examined numerically, an analytic extension to higher $\mathrm{E}$ values was performed by assuming that the break in wavenumber slope $\left(\mathrm{m}_{\mathrm{c}}\right)$ occurred where the inverse Richardson number $\left\langle\mathrm{S}^{2}>/ \mathrm{N}^{2}\right.$ reached .5 (the "critical Richardson number hypothesis", $\mathrm{Em}_{\mathrm{c}}=$ constant) The energy fluxes were calculated at a vertical wavenumber twice this cutoff $\mathrm{m}_{\mathrm{v}}=2 \mathrm{~m}_{\mathrm{c}}$. For the GM energy levels, $\mathrm{m}_{\mathrm{c}}=.1 \mathrm{cpm}$ and $\mathrm{m}_{\mathrm{v}}=.2 \mathrm{cpm}$. Moreover, HWF chose to neglect the vertical component of the background flow, with the justification that its inclusion would lead to a "spurious decorrelation" between the test wave and the critical 
layer represented by the background flow. Consistency requires that the vertical component be dropped in the conjugate equation. This term is of the form $\delta(w \mathrm{~m}) / \delta \mathrm{z}$ and represents the vertical straining of the ambient stratification by the background wave field. A related approximation is the use of the ambient stratification in the dispersion relation. Henyey et al. rather convincingly argue that the neglect of the potential energy term is energetically inconsequential.

From the numerical simulations it is noted in the high wavenumber part of the domain the transport is governed by

$$
\frac{\mathrm{d} \mathrm{m}}{\mathrm{d} \mathrm{t}} \sim-\frac{\delta \mathbf{u} \cdot \mathbf{k}}{\delta \mathrm{z}} .
$$

Dissipation is defined as $\varepsilon=\langle\mathrm{d} \hat{E} / \mathrm{dt}\rangle=\langle(\mathrm{dE} / \mathrm{dm})(\mathrm{dm} / \mathrm{dt})\rangle$. Since $\mathrm{dm} / \mathrm{dt}$ can be either up or down scale, it is estimated as $|\mathrm{dm} / \mathrm{dt}|(1-$ $\mathrm{r}) /(1+\mathrm{r})$, where the factor $\mathrm{r}$ (the ratio between up and down scale energy fluxes) is introduced. From the numerical simulations a value of $\mathrm{r}=.4$ at $\mathrm{m}=\mathrm{m}_{\mathrm{v}}$ is chosen. The eikonal equation is then used to estimate $|\mathrm{dm} / \mathrm{dt}|: \quad|\mathrm{dm} / \mathrm{dt}|=|\delta(\mathbf{u} \cdot \mathbf{k}) / \delta \mathrm{z}|=\left(\left\langle\mathrm{u}_{\mathrm{z}}^{2}>\mathrm{k}_{\mathrm{h}}^{2}\left\langle\cos ^{2} \theta>\right)^{1 / 2}=\right.\right.$ $\mathrm{Nk}_{\mathrm{h}}\left(<\mathrm{Ri}^{-1}>/ 2\right)^{1 / 2}$. At $.2 \mathrm{cpm},<\mathrm{Ri}^{-1}>=\mathrm{Ri}_{\mathrm{c}}^{-1}\left\{1+\ln \left(\mathrm{m}_{\mathrm{v}} / \mathrm{m}_{\mathrm{c}}\right)\right\}$ where $\mathrm{Ri}_{\mathrm{c}}^{-1}=$ .5 and $\mathrm{a} \mathrm{m}^{-1}$ slope to the shear spectra past $\mathrm{m}_{\mathrm{c}}$ is explicitly assumed. The term $\mathrm{dE} / \mathrm{dm}$ is merely the energy density at the small scale end of the spectrum. Thus the dissipation is proportional to the shear at the small scale end of the spectrum. Hence large shears lead to large transports and dissipations for this model. From the numerical simulations, HWF note that the presence of critical layers (regions in which the wavenumber of the test wave exceeds $.2 \mathrm{cpm}$ ) are well correlated with large shear events in the background flow (Henyey, 
1984). We wish to exploit this property of the model in Chapter Four in conjunction with the omission of the straining of the ambient stratification by the background flow discussed above. The expression is evaluated at $\mathrm{m}_{\mathrm{v}}$ under the explicit assumption of the 'critical Richardson number hypothesis' using a GM spectrum modified at large wavenumbers. The answer is:

$$
\varepsilon=\frac{d \hat{E}}{d t}=.33 \mathrm{f}\left[\frac{4 \mathrm{j}_{*}}{\pi} \mathrm{bNE}\right]^{2} \cosh ^{-1}\left(\frac{\mathrm{N}}{\mathrm{f}}\right) .
$$

At $32.5 \mathrm{~N}, \varepsilon\left(\mathrm{E}_{\mathrm{GM}}, \mathrm{N}_{\mathrm{o}}\right)=3.4 \times 10^{-10}(\mathrm{~W} / \mathrm{kg})$.

Henyey et al. point out that the wave-wave interactions in the eikonal approach are different in character than the id or psi mechanisms. Those mechanisms resulted in energy fluxes to lower frequency. For the model of HWF the energy fluxes are towards higher frequency. From the dispersion relation this implies fluxes to larger horizontal wavenumbers, in clear distinction from the id mechanism where the energy fluxes occur at constant horizontal wavenumber.

The frequency scaling of the HWF model is easily approached (Henyey, 1991). From eq. 3.4, $\varepsilon \sim \mathrm{k}_{\mathrm{h}}<\mathrm{u}_{\mathrm{z}}^{2}>^{1 / 2}(\mathrm{dE} / \mathrm{dm})$ evaluated at $\mathrm{m}_{\mathrm{v}}$. Under the universal Richardson number hypothesis, $\left\langle u_{z}^{2}\right\rangle^{1 / 2}$ scales as $\mathrm{N}$ (see Appendix Two for further details). The energy density scales as $\mathrm{N}^{2} \mathrm{E} / \mathrm{m}^{2}$ for a $\mathrm{GM}$ vertical wavenumber dependance, and for hydrostatic internal waves $\mathrm{k}_{\mathrm{h}}=\left(\omega^{2}-\mathrm{f}^{2}\right)^{1 / 2} \mathrm{~m} / \mathrm{N}$. Putting this together and recognizing $\mathrm{Em}_{\mathrm{c}}$ is a constant produces:

$$
\varepsilon \sim \mathrm{N}^{2} \mathrm{E}^{2}\left(\omega^{2}-\mathrm{f}^{2}\right)^{1 / 2} .
$$

The scalings for id and the model of HWF are thus different only at near-inertial frequencies. 


\section{Gargett and Holloway (1984) GH}

Much of the actual dissipation data preceding $\mathrm{GH}$ indicated an inverse buoyancy scaling of $\varepsilon / \mathrm{N}^{2}$, i.e. $\mathrm{K}_{\rho}=.25 \varepsilon / \mathrm{N}^{2}$ increasing with depth as $\mathrm{N}$ decreased. Little or no information about internal wave energy levels was collected with the dissipation estimates and it was therefore assumed that the internal wave energy levels were roughly at GM levels. Hence GH sought to explain an apparent inverse scaling of $\varepsilon / \mathrm{N}^{2}$ at constant internal wave energy levels. To do this they avoided the classical Reynolds decomposition but yet retained a turbulent production-dissipation balance in the turbulent kinetic energy equation:

$$
<\mathrm{uw} \frac{\delta \mathrm{u}}{\delta \mathrm{z}}>=-\frac{\mathrm{g}}{\rho}<\mathrm{w} \rho>-\varepsilon .
$$

Under the assumption of a small flux Richardson number,

$$
\mathrm{R}_{\mathrm{f}}=\frac{\mathrm{g}}{\rho}<\mathrm{w} \rho>K \mathrm{uw} \frac{\delta \mathrm{u}}{\delta \mathrm{z}}>\text { (usually } \mathrm{R}_{\mathrm{f}} \text { is taken to be } \leq .2 \text { ), }
$$

this then becomes $\quad\langle\mathrm{uw}(\delta \mathrm{u} / \delta \mathrm{z})>\sim-\varepsilon$.

The unique part of GH's work comes from the observation that there is no clear vertical scale separation in velocity between internal wave scales and the scales at which correlations leading to a turbulent momentum flux occur. They asserted that if these scales are dominated by internal wave like motions, then the variables in the momentum flux ought to retain their internal wave (nominally WKB) scalings. (For a detailed discussion of the WKB and GM spectral scalings, see Appendix Two and Section 3.3.)

For a single wave; $\left\langle\mathrm{u}^{2}\right\rangle \sim \mathrm{N},\left\langle\mathrm{w}^{2}\right\rangle \sim \mathrm{N}^{-1}$, and $\left\langle\mathrm{u}_{\mathrm{z}}^{2}\right\rangle \sim \mathrm{N}^{3}$. However, under the assumption that a wave would be Richardson number 
limited, $\left\langle\mathrm{u}_{\mathrm{z}}^{2}\right\rangle \sim \mathrm{N}^{2}$. Assuming the correlation coefficient in the turbulent momentum flux term to be independent of $\mathrm{N}$ results in $\varepsilon \sim \mathrm{N}^{+1}$. For the GM internal wave spectrum, $\left\langle\mathrm{u}^{2}\right\rangle \sim \mathrm{N}$ as before, but due to the decreasing frequency bandwidth $\left\langle\mathrm{w}^{2}\right\rangle \sim \mathrm{N}^{0}$. If the cutoff wavenumber of the shear spectrum fails to scale with $\mathrm{N}$, then $\left\langle\mathrm{u}_{\mathrm{z}}^{2}\right\rangle \sim \mathrm{N}^{2}$ as the shear spectrum scales with $\mathrm{N}^{2}$. Hence $\varepsilon \sim \mathrm{N}^{+3 / 2}$ for a GM wave field (see Appendix Two). A similar and consistent analysis was performed by $\mathrm{GH}$ for the heat equation.

An energy level scaling can be incorporated into the $\mathrm{GH}$ formalism in the following manner: If $\mathrm{Em}_{\mathrm{c}}$ is a constant, then $\left\langle\mathrm{u}_{\mathrm{z}}^{2}\right\rangle$ at some wavenumber $\gg m_{c}$ varies only weakly with $E$, i.e. as $\ln (E)$ for a $m^{-1}$ spectrum above $\mathrm{m}_{\mathrm{c}}$. Hence $\left\langle\mathrm{u}_{\mathrm{z}}^{2}\right\rangle \sim \mathrm{N}^{\circ} \mathrm{E}^{\circ}$. Since $\left\langle\mathrm{u}^{2}\right\rangle$ and $\left\langle\mathrm{w}^{2}\right\rangle$ scale as $\mathrm{E}$,

$$
\varepsilon \sim \mathrm{EN}^{+3 / 2} .
$$

This extension appeared in Gargett (1990) as part of a response to the claims of Gregg (1989), which is discussed below in detail.

\section{Munk (1981)}

Munk (1981) provided the earliest model linking internal waves to dissipation. The model is purely kinematic, linking the time rate of change of energy to the rate of occurrence of instabilities. $\mathrm{He}$ assumed that $\mathrm{d} \hat{\mathrm{E}} / \mathrm{dt}=-\sigma \hat{\mathrm{E}} \mathrm{p}(\phi>1)$, where $\sigma$ is the frequency of occurrence per unit time of an unstable event, $\hat{E}$ is the energy, $\phi$ is an instability parameter, and $\mathrm{p}(\phi>1)$ represents the probability of an instability criterion being exceeded. Gaussian statistics are assumed for $\phi, \mathrm{p}(\phi>1)=\left(2<\phi^{2}>/ \pi\right)^{1 / 2} \exp \left(-1 / 2<\phi^{2}>\right)$. If $\phi$ is assumed to represent shear instability, $\phi=\mathrm{u}_{\mathrm{z}} / 2 \mathrm{~N}$. The time scale is assumed to be the 
fastest available, that of the strain field, $\sigma=\sqrt{\mathrm{fN}} / \pi$. This results in a scaling for $\varepsilon$ of $\mathrm{f}^{1 / 2} \mathrm{~N}^{3 / 2} \mathrm{E}^{3 / 2} \exp$ (-const/E) if the cutoff wavenumber is taken to be independent of E (Gregg, 1989). Dissipations predicted for this model by Gregg (1989) are large; at $\mathrm{N}=3 \mathrm{cph}, \varepsilon=1.3 \times 10^{-8}$ W/kg. However, use of values consistent with Munk (1981) reduces this to $3.4 \times 10^{-9} \mathrm{~W} / \mathrm{kg}$, comparable to the McComas and Muller predictions (eq. 3.2). The exponential scaling of $\mathrm{E}$ produces predictions for dissipation which are much too large at high E (Gregg 1989, Gargett 1990). This dependance is reduced significantly if the break in slope of the shear spectrum is taken into account. For energy levels greater than GM, evaluation of the shear instability parameter at a constant length scale of $(.1 \mathrm{cpm})^{-1}$ produces $\left.<\phi^{2}\right\rangle=\left(1+\ln \left(\mathrm{E}_{\mathrm{E}} \mathrm{E}_{\mathrm{GM}}\right)\right) / 8$. The expression for the dissipation becomes:

$\varepsilon=-\frac{\mathrm{d} \hat{\mathrm{E}}}{\mathrm{dt}}=\hat{\mathrm{E}} \frac{\sqrt{\mathrm{fN}}}{\pi}\left(\frac{\left(1+\ln \left(\mathrm{E} / \mathrm{E}_{\mathrm{GM}}\right)\right)}{4 \pi}\right)^{1 / 2} \exp \left\{-4 /\left(1+\ln \left(\mathrm{E} / \mathrm{E}_{\mathrm{GM}}\right)\right)\right\}$.

For $E / E_{G M}>2$, the scaling of this expression is approximately $\mathrm{f}^{1 / 2} \mathrm{~N}^{3 / 2}$ $\mathrm{E}^{2}$. It may be possible to include a frequency dependence in the strain time scale. However, we are able in the following to eliminate the above scaling on the basis of the buoyancy frequency dependence and thus neglect to extend this model. 
3.3 The criticism of Gregg (1989) by Gargett (1990)

Ann Gargett has criticized the work of Gregg (1989):

This paper questions a recent claim by Gregg (1989) that a first-order understanding of the link between internal waves and turbulence has been achieved. It is demonstrated that (1) the theoretical basis of this result is extremely sensitive to an ad hoc assumption about the nature of the wavelength at which the wave energy is delivered to dissipation (turbulence), and (2) the method used by Gregg (1989) to calculate instantaneous wave field energy level $\mathrm{E}$ is incorrect and will seriously underestimate $\mathrm{E}$ in cases where $\mathrm{E}$ is greater than $\mathrm{E}_{\mathrm{GM}}$, the energy level of the $\mathrm{GM}$ (Garrett and Munk, 1975) canonical internal wave field, and (3) the range of variation of $\mathrm{E}$ and buoyancy frequency $\mathrm{N}$ in the data sets reported by Gregg (1989) is not sufficient to rule out alternative scalings.

Gargett suggests that the cutoff wavenumber used by Henyey et al. $\left(\mathrm{m}_{\mathrm{v}}=2 \mathrm{~m}_{\mathrm{c}}\right)$, that wavenumber where the test waves were declared to 'break' and the ray tracing was terminated, has "no physical meaning in the oceanic context". She suggests that an Osmidov length would perhaps be more relevant and would result in quite a different scaling relationship:

$$
\begin{aligned}
& \varepsilon=\frac{3 \pi \mathrm{Ri}_{\mathrm{c}}^{1 / 2}}{4 \mathrm{f}}\left(\frac{4}{\pi} \mathrm{j} * \mathrm{bNEf}\right)^{2}\left(\frac{\mathrm{m}_{\mathrm{c}}}{\mathrm{m}_{\mathrm{v}}}\right)^{2}\left(\frac{1+\ln \left(\frac{\mathrm{m}_{\mathrm{c}}}{\mathrm{m}_{\mathrm{v}}}\right)}{2}\right)^{1 / 2} \frac{1-\mathrm{r}}{1+\mathrm{r}} \cosh ^{-1}\left(\frac{\mathrm{N}}{\mathrm{f}}\right) \\
& \mathrm{L}_{\mathrm{o}} \sim\left(\frac{\varepsilon}{\mathrm{N}^{3}}\right)^{1 / 2} \text { for } \mathrm{m}_{\mathrm{v}} \text { and } \mathrm{r}=.4 .
\end{aligned}
$$

This complaint has no basis as the vertical scale at which the ray tracing is terminated need have no physical meaning. For a isotropic, homogeneous, stationary wave spectrum the flux past a given wavenumber should give an accurate estimate of the dissipation as long as generation occurs at larger and dissipation at smaller scales than the wavelength in question. The requirement of stationarity implies that the energy flux through the wavenumber spectrum is 
independent of wavenumber and thus the energy flux can be equated with the dissipation and estimated at any convenient wavenumber. The estimate of dissipation as:

$$
\varepsilon=\left\langle\frac{\mathrm{dE}}{\mathrm{dm}} \frac{\mathrm{d} \mathrm{m}}{\mathrm{dt}}\right\rangle, \frac{\mathrm{dm}}{\mathrm{d} \mathrm{t}}=-\frac{\delta(\mathbf{u} \cdot \mathbf{k})}{\delta \mathrm{z}}
$$

requires that the fluxes in vertical wavenumber be dominated by $\mathrm{dm} / \mathrm{dt}=-\delta(\mathbf{u} \cdot \mathbf{k}) / \delta \mathrm{z}$. From the numerical simulations, Henyey et al. assures us that this is the case and that the test wave spectrum is in equilibrium with the GM background vertical wavenumber spectrum (i.e. is stationary). Hence eq. 3.9 ought to be an adequate parameterization of the model fluxes. However, one may be concerned that the value of $\mathrm{m}_{\mathrm{v}}$ is too large. At $\mathrm{m}_{\mathrm{v}}$, models of Richardson number statistics (Chapter Four) predict about 9\% Ri < 1/4. If there are significant dissipations associated with shear instabilities at these scales, the model may not be an accurate representation of the actual physical processes occurring in the ocean.

On the other hand, the second and third of Gargett's points are well taken. Both Henyey et al. and the extension of GH assume that $\mathrm{Em}_{\mathrm{c}}=$ constant. Gregg (1989) seems to have realized this in the case of Henyey et al., but failed to provide estimates of $\mathrm{E}$ consistent with the model, and in doing so consistently underestimates large values of $\mathrm{E}$.

The point is simple but bears discussion. Further details can be found in Appendix Two. WKB scaling requires

$$
\mathrm{u}_{\mathrm{z}}^{2} \sim \mathrm{N}^{3}, \mathrm{~m} \sim \mathrm{N} .
$$

The vertical wavenumber shear spectral density, $\mathrm{S}_{\mathrm{z}}$, scales as $\mathrm{N}^{2}$ and 


$$
\int_{0}^{m^{\prime}} S_{z} d m \sim N^{3}
$$

if m' WKB scales. From an observational standpoint (Gargett et al., 1981; Duda and Cox, 1989; Gregg et al., 1991; Figure 3.5), there is a tendency for the shear spectral density to be constant up to some wavenumber $\mathrm{m}_{\mathrm{c}}$ and then to decrease as $\mathrm{m}^{-1}$ (see Figure $3.1 \mathrm{a}$ ). There is evidence that the cutoff wavenumber, $\mathrm{m}_{\mathrm{c}}$, is a simple function of both the buoyancy frequency $(\mathrm{N})$ and the energy level (E). At constant buoyancy scaled energy levels (E) there is evidence that $\mathrm{m}_{\mathrm{c}}$ is constant, i.e. $\mathrm{m}_{\mathrm{c}}$ does not WKB scale (Gargett et al. 1981) so that

$$
\int_{0}^{\mathrm{m}_{\mathrm{c}}} \mathrm{S}_{\mathrm{Z}} \mathrm{dm} \sim \mathrm{N}^{2} .
$$

If the cutoff wavenumber, $\mathrm{m}_{\mathrm{c}}$, is taken to be $\sim .1 \mathrm{cpm}$, the Munk ' 81 spectrum implies

$$
\int_{0}^{\mathrm{m}_{\mathrm{c}}} \mathrm{S}_{\mathrm{z}} \mathrm{dm}=\mathrm{N}^{2} / 2
$$

(see Figure 3.1 b). Gargett (1990) sites Duda and Cox (1989) (see also Gregg et al., 1991 and Figure 3.12) as providing additional evidence that the cutoff wavenumber scales inversely with $\mathrm{E}$, so that $\mathrm{Em}_{\mathrm{c}}$ is constant (see Figure $3.1 \mathrm{c}$ ). Under such a scaling, the integration of the shear spectral density out to $m_{c}$ results in $\left\langle S^{2}>/ N^{2}=1 / 2\right.$, independent of N and E. While both Duda and Cox (1989) and Gregg et al. (1991) note qualitative agreement with the universal Richardson number hypothesis, suggesting that the universal shear spectrum suggested by Gargett (1990) may provide a zeroth order description, both Duda and Cox (1989) and Gregg et al. (1991) point 
A
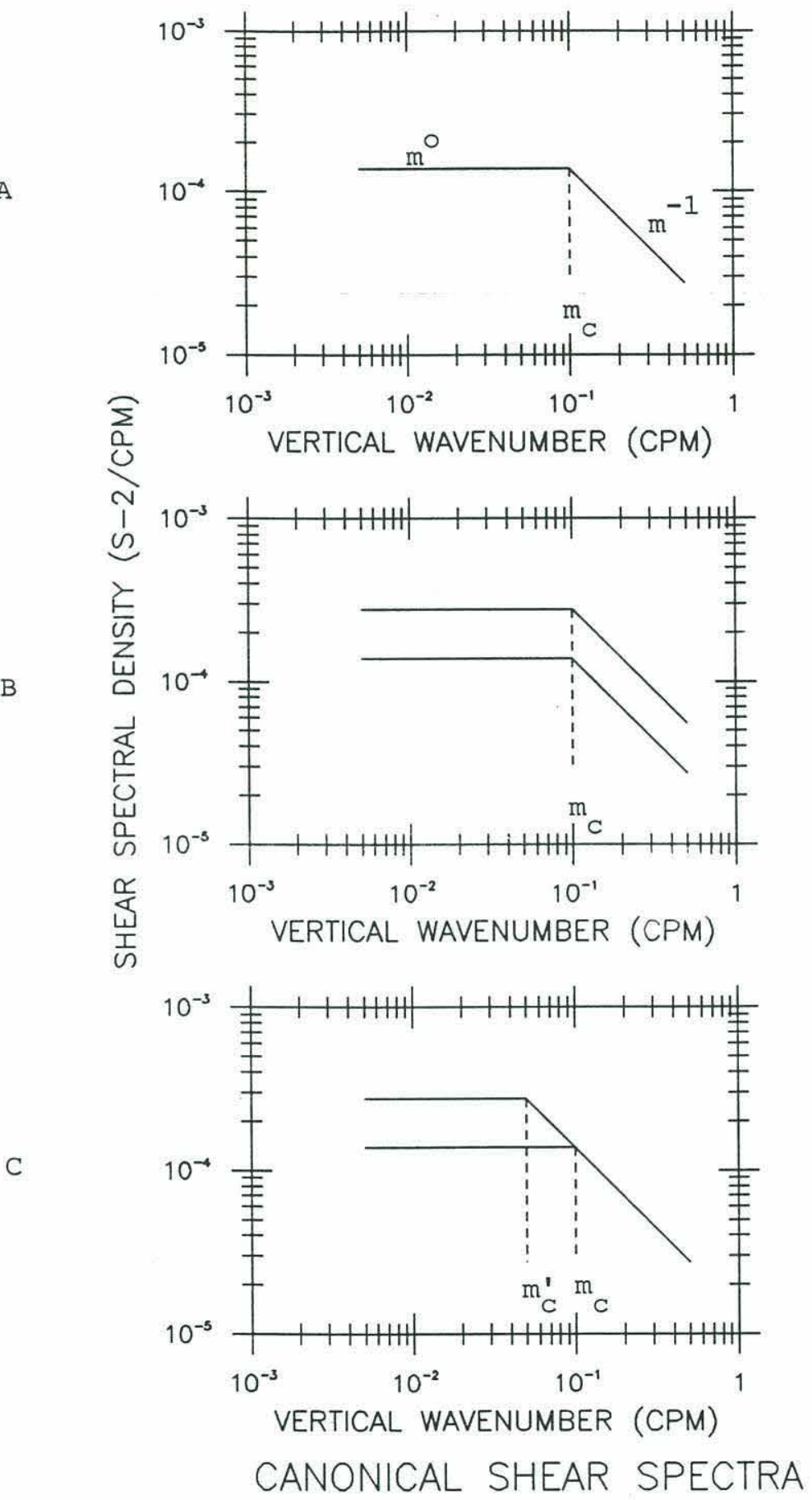

Figure 3.1: Canonical scaling of the shear spectral density, $S_{z}$. The canonical form of $S_{z}$ is taken to be a simple form of $E$ and $N$. a) In general, $S_{z}$ is white up to some cutoff wavenumber $m_{c}$ and then falls as $\mathrm{m}^{-1}$. b) $\mathrm{S}_{\mathrm{z}}(\mathrm{N})$ : On the basis of Gargett et. al., 1981, the vertical cutoff wavenumber is taken to be independent of N. WKB scaling requires $S_{z} \sim N^{2}$. c) $S_{z}(E)$ : On the basis of Duda and Cox (1989), $m_{c}$ scales inversely with $\mathrm{E}$. 
to quantitative disagreements. In Chapter Four a number of dynamically based speculations are forwarded which may potentially explain the observed variability in the shear spectra. The possible quantitative disagreements noted by Duda and Cox (1989) and Gregg et al. (1991) do not affect the empirical results contained herein. Gregg (1989) estimated $\mathrm{E}^{2}$ by averaging estimates of a ten meter first difference shear raised to the fourth power. The first difference estimate has a half power point at $.05 \mathrm{cpm}$, and estimates of the shear variance with wavelengths larger than $1 / .1 \mathrm{cpm}$ were produced by multiplying by a factor of 2.11. (See also Appendix One and Figure A.1.3) In the models discussed in Section 3.2, E is taken to be a nondimensional spectral level for wavenumbers less than $\mathrm{m}_{\mathrm{c}}$. If the shear spectrum is white, one method of estimating $\mathrm{E}$ is to determine the average spectral level from the spectrum itself. We have chosen to do so in the study which follows. Since the integral of the shear spectral density is the shear variance, another method of obtaining an estimate of $\mathrm{E}$ is to assume that the spectrum is white out to a constant dimensional wavenumber, calculate the shear variance in the spatial domain and then divide by the average bandwidth of the estimate. Gregg choose the later method. This estimate of $\mathrm{E}$ will tend to underestimate the average spectral density below $\mathrm{m}_{\mathrm{c}}$ if the half-power point of the first difference transfer function occurs at a wavenumber larger than $\mathrm{m}_{\mathrm{c}}$. Consider the following example: if $S_{z}$ is white out to $m_{c}$ and then falls as $\mathrm{m}^{-1}$, one obtains, in the case that $\mathrm{m}_{\mathrm{c}}<.05 \mathrm{cpm}$ (the half-power point of the ten meter first difference operator), an estimate of the shear spectral density from the spatial domain $\left(S_{z}^{f d}\right)$ of: 


$$
\mathrm{m}_{\mathrm{c}} \mathrm{S}_{\mathrm{z}}\left\{1+\ln \left(\frac{.05}{\mathrm{~m}_{\mathrm{c}}}\right)\right\} 2.11 / .1=\mathrm{S}_{\mathrm{z}}^{\mathrm{fd}} \text { for } \mathrm{m}_{\mathrm{c}}<.05 \mathrm{cpm}
$$

Gargett points out that if, say, $\mathrm{E} / \mathrm{E}_{\mathrm{GM}}=6\left(\mathrm{~m}_{\mathrm{c}}=.1 / 6\right)$, then the average shear spectral density between 0 and $.05 \mathrm{cpm}$ is $\mathrm{S}_{\mathrm{z}}^{\mathrm{fd}}=.70 \mathrm{~S}_{\mathrm{z}}$ and thus $\mathrm{E}^{2}$ estimates will be biased low by a factor of two. If $\mathrm{E} / \mathrm{E}_{\mathrm{GM}}$ is ten, the bias is approximately a factor of four.

Gregg claims to have collapsed his data to within a factor of two using the Henyey et al. $\mathrm{E}^{2} \mathrm{~N}^{2}$ scaling. Gargett uses much of the same data to show that, given the consistent bias at high $\mathrm{E}$, the data is not inconsistent with $\mathrm{EN}^{3 / 2}$, the extension of $\mathrm{GH}$, to within the same factor of two. Given the bias problems at high E, one is led to conclude that the $\mathrm{E}$ dependance has not been adequately tested. If one assumes that the range of $\mathrm{N}^{2}$ in the PATCHEX experiment is similar to the whole of Gregg's data sets (see Gargett 1990), one runs into concurrent problems with testing the $\mathrm{N}^{2}$ dependance. The ratio of $\mathrm{E}^{2}$ to $\mathrm{E}_{\mathrm{GM}}^{2}$ that Gregg reports for PATCHEX was $<4$, so that the scaling should not suffer from the bias problems in the E scaling. Hence PATCHEX should be a good data set to test the $\mathrm{N}$ dependance. However, if one excludes the upper $20 \mathrm{~m}$ of the data, the range in $\mathrm{N}$ is merely $.25 \times 10^{-2}<\mathrm{N}<1.4 \times 10^{-2}$, or a difference in $\mathrm{N}^{1 / 2}$ of 2.4. Given the factor of two variability in the scaled data, one cannot fairly claim to have tested between an $\mathrm{N}^{2}$ or $\mathrm{N}^{3 / 2}$ dependance (see Figures $3.2 \& 3.3)$.

Figures 3.2 and 3.3 are abstracted from Gargett (1990) (left panels) and from Gregg (1989) (right panels). The right hand panels consist of averaged PATCHEX dissipations divided by Gregg's internal 


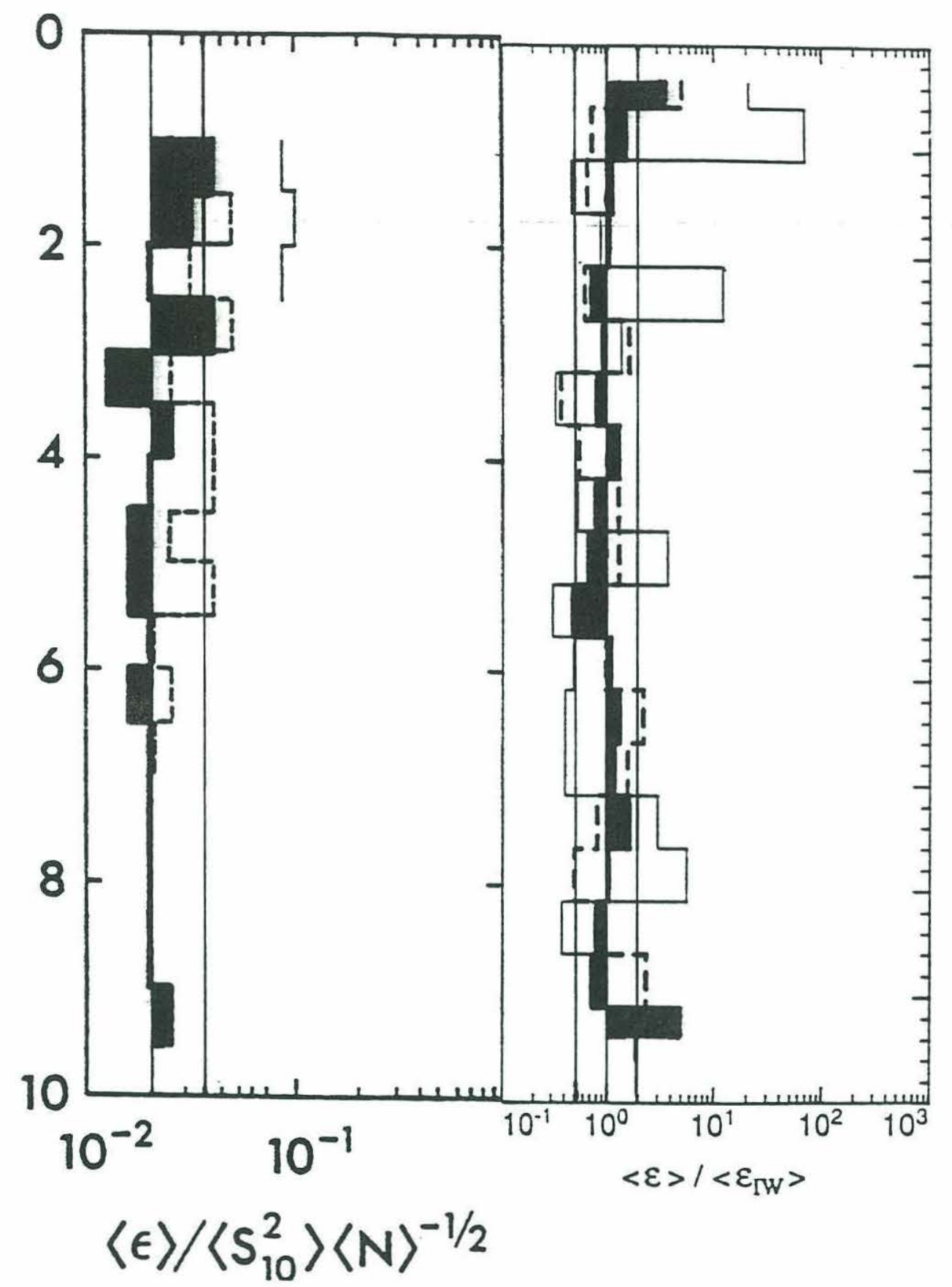

Figure 3.2: Comparison of the scaling of PATCHEX data from Gregg(1989) (right hand panel) and Gargett (1990) (left hand panel). The PATCHEX curves have been colored in about $\langle\varepsilon\rangle \mid\left\langle\varepsilon_{\text {iw }}\right\rangle=1$ in the case of Gregg and $\langle\varepsilon\rangle \mid\left\langle\mathrm{S}_{0}^{2}\right\rangle\langle\mathrm{N}\rangle^{-1 / 2}=.02$ in the case of Gargett. The thin lines represent a factor of two about these values. There appears to be little difference between the scalings. The remaining lines represent other data sets. 


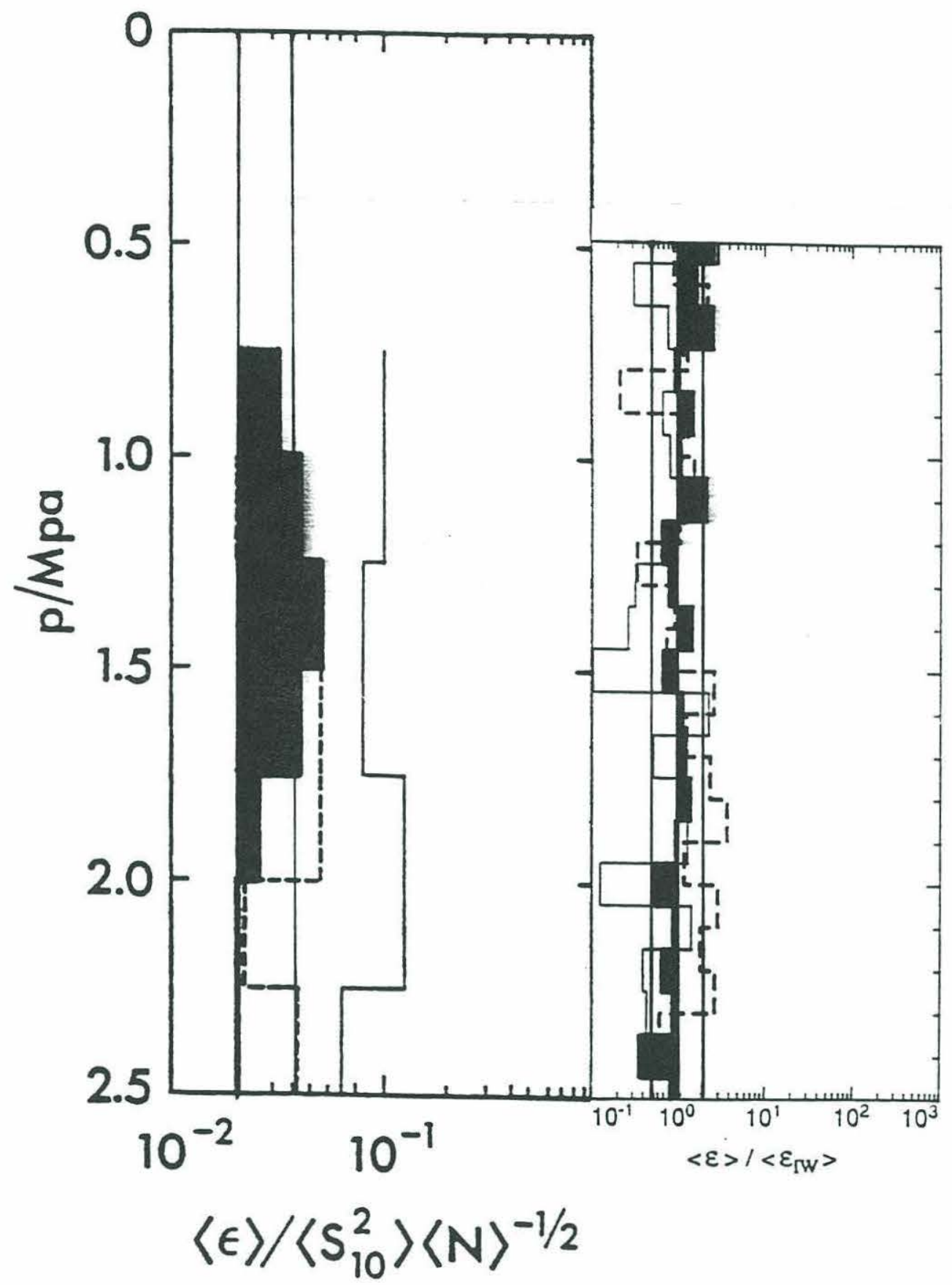

Figure 3.3: As in figure 3.2 excepts for $50-250 \mathrm{db}$. 
wave scaling, $\varepsilon_{I W}$, which has a nominal scaling of $\mathrm{E}^{2} \mathrm{~N}^{2}$. The left hand panels are the same dissipations divided by $<\mathrm{S}_{10}^{2}>\mathrm{N}^{-1 / 2}$, or $\mathrm{EN}^{3 / 2}$, where $\left\langle\mathrm{S}_{10}^{2}\right\rangle$ is an average 10 meter first difference estimate of the shear variance which should scale as $\mathrm{N}^{2}$. Figure 3.2 presents data from $50-1000 \mathrm{db}$, Figure 3.3 presents data from $50-250 \mathrm{db}$. It is difficult to assert from these figures that $\mathrm{E}^{2} \mathrm{~N}^{2}$ collapses the PATCHEX data better than $\mathrm{EN}^{3 / 2}$.

\subsection{Data Analysis}

Before examining the dissipation scalings we wish to briefly discuss the procedure for estimating the various parameters in the analysis. Further details can be found in Appendix One. The models discussed previously reference a shear and/or energy density spectral level, an average buoyancy frequency, and various expressions involving the frequency content of the internal wave field. We refer to both the shear and energy density as a nondimensional energy level and estimate this level from averaged estimates of the shear spectra for wavenumbers less than the cutoff vertical wavenumber, $m_{c}$. The average buoyancy frequencies are determined from averaged profiles of temperature and salinity. The expressions involving frequency content are determined from the ratio between the shear and strain spectra for wavenumbers less than $\mathrm{m}_{\mathrm{c}}$.

The symbol $S_{z}$ represents an averaged estimate of the shear spectral density normalized to $3 \mathrm{cph}$ for wavenumbers less than $\mathrm{m}_{\mathrm{c}}$, the cutoff vertical wavenumber where 


$$
\int_{0}^{\mathrm{m}_{\mathrm{c}}} \mathrm{S}_{\mathrm{Z}} \mathrm{dm}=\mathrm{N}^{2} / 2
$$

The estimate for $S_{z}$ was produced by integrating the averaged spectral estimates until $\mathrm{N}_{\mathrm{o}}^{2} / 2$ was exceeded, and then dividing by the bandwidth of the estimate, $\mathrm{m}_{\mathrm{c}}$, (the bandwidth being (the number of estimates in the sum) $x$ (number of points in the transform $x$ decimation interval) $)^{-1}$ of the estimate). The upper limit of integration was chosen on the basis of the Munk (1981) spectra:

$$
\int_{0}^{m_{c}} m^{2} E(m) d m \sim 1.38 \times 10^{-4} \frac{\mathrm{s}^{-2}}{\mathrm{cpm}} \times .1 \mathrm{cpm}\left(\text { i.e. } \mathrm{m}_{\mathrm{c}}^{2} \times E\left(\mathrm{~m}_{\mathrm{c}}\right) \times \mathrm{m}_{\mathrm{c}}\right)=\mathrm{N}^{2} / 2
$$

The average strain spectral density, $F_{z}$, represents an average over wavenumbers less than $\mathrm{m}_{\mathrm{c}}$, where $\mathrm{m}_{\mathrm{c}}$ was determined from the shear spectrum as described above. Further details can be found in Appendix One.

The procedure of buoyancy (WKB) scaling references a background buoyancy frequency in a quiescent ocean. In this study, $\mathrm{N}$ is estimated from the average values of temperature and salinity on a pressure surface from a group of stations. Those average profiles are used to produce 20 meter least square fit estimates of $\mathrm{N}^{2}$. This procedure is employed to avoid the variability in $\mathrm{N}^{2}$ due to internal wave straining, which is quite large in highly energetic wave fields. The value of $\mathrm{N}$ used to normalize the shear spectra was obtained from the averages of $\mathrm{N}$ over the relevant depth interval.

The models require two expressions involving the frequency content to be estimated. It is relatively routine to derive an 
expression for the ratio between the shear $\left(\mathrm{S}_{\mathrm{z}}\right)$ and strain $\left(\mathrm{F}_{\mathrm{z}}\right)$ spectra in the case of no mean flow:

$$
\frac{S_{z}}{N^{2} F_{z}}=\frac{\left(\omega^{2}+f^{2}\right)\left(N^{2}-\omega^{2}\right)}{\left(\omega^{2}-f^{2}\right) N^{2}}=r_{\omega}
$$

This methodology was first used by Kunze, Williams and Briscoe (1990) and follows directly from Fofonoff (1969) since the shear to strain ratio is identical the the horizontal kinetic energy to available potential energy ratio. For the GM spectra, $r_{\omega}=3$ (Appendix Two). If $\delta \mathrm{t}$ is simply replaced by $\delta \mathrm{t}+\mathrm{U}_{0} \delta \mathrm{x}$ in the equations of motion, the same expression for the shear/strain ratio results if $\omega$ is interpreted as the intrinsic frequency. The ratio $r_{\omega}$ is calculated by averaging the shear and strain spectra over wavenumbers less than $\mathrm{m}_{\mathrm{c}}$ and then dividing. The squared frequency is calculated by solving the quadratic represented in equation 3.10:

$$
\omega^{2}=\left(N^{2}\left(1-r_{\omega}\right)-f^{2}+\left(N^{4}\left(r_{\omega}-1\right)^{2}+2 N^{2} f^{2}\left(1+3 r_{\omega}\right)+f^{4}\right)^{1 / 2}\right) / 2 .
$$

The ratio between horizontal and vertical wavenumbers required for the frequency dependent correction to the HWF model (eq. 3.6) is determined from a similar quadratic expression:

$$
\frac{\mathrm{k}_{\mathrm{h}}^{2}}{\mathrm{~m}^{2}}=\frac{\left(\omega^{2}-\mathrm{f}^{2}\right)}{\left(\mathrm{N}^{2}-\omega^{2}\right)}=\frac{-\mathrm{r}_{\omega}+1+\frac{\mathrm{f}^{2}}{\mathrm{~N}^{2}}+\left(\left(\mathrm{r}_{\omega}-1-\frac{\mathrm{f}^{2}}{\mathrm{~N}^{2}}\right)^{2}+8 \mathrm{r}_{\omega} \frac{\mathrm{f}^{2}}{\mathrm{~N}^{2}}\right)^{1 / 2}}{2 \mathrm{r}_{\omega}} .
$$

This expression represents a spectrally weighted estimate of the frequency content. 


\subsection{Model/Data Comparison}

Here we attempt to resolve the question of an appropriate $(\mathrm{E}, \mathrm{N},\langle\omega\rangle)$ scaling for the rate of kinetic energy dissipation, $\varepsilon$. To do so we attempt to address the $(\mathrm{E},\langle\omega\rangle)$ and $\mathrm{N}$ scaling problems as separately as possible. The $\mathrm{N}$ scaling is addressed using $83000 \mathrm{db}$ profiles from TOPO. These profiles exhibited little variation in E or $\langle\omega\rangle$ and have a large variation in N. The E and $\langle\omega\rangle$ scaling is examined with the inclusion of 15 profiles from a region of steep topography and 40 profiles from the middle of a warm core ring. The $\mathrm{E}$ values in the later two data sets tend to be larger than the first set. The E values from the ring tend to be the largest and have a lower frequency content, the profiles from the region of steep topography tend to have the highest frequency content.

\section{The $\mathrm{N}$ dependence}

We choose to examine the question of buoyancy scaling by analyzing a subset of data which spanned a large range in $\mathrm{N}$ but a small range in E. To do so we have selected eight profiles from the vicinity of Fieberling Guyot $\left(32.5^{\circ} \mathrm{N}, 128^{\circ} \mathrm{W}\right)$. These profiles were taken in April of 1991 as part of the abrupt topography initiative (TOPO). The profiles in question were taken at a distance of $20-40 \mathrm{~km}$ from the center of the seamount, in $3400-4000 \mathrm{~m}$ of water (Figure 3.4). Large-scale background flows were observed to be less than $2 \mathrm{~cm} / \mathrm{s}$ at the time of the experiment (J. Toole, personal communication). Of the eight profiles, three of them proved to be on the flank of the seamount and exhibited heightened turbulent 
70

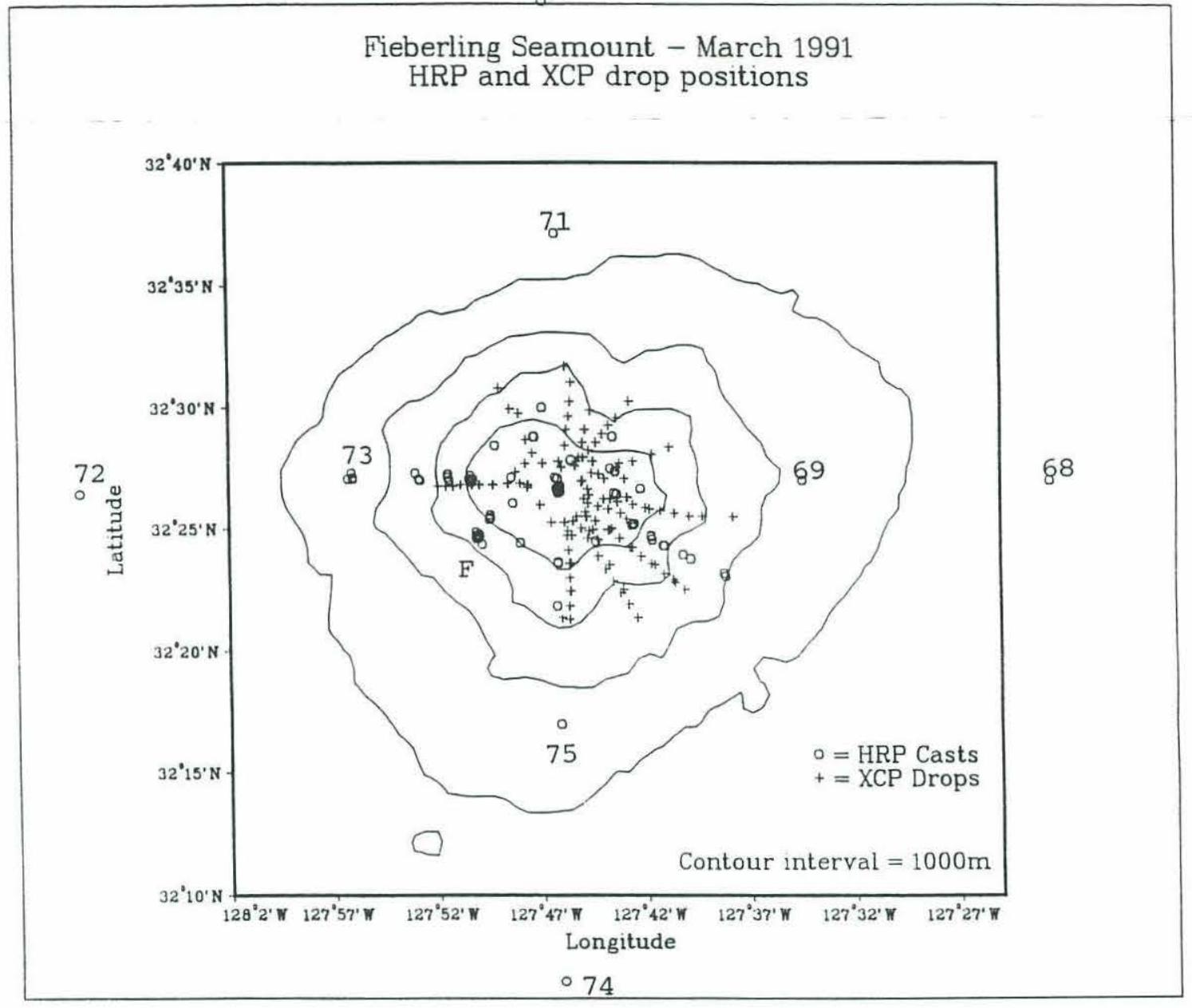

Figure 3.4: Bathymetry and positions of profiler deployments around Fieberling Guyot. The eight deep profiles are denoted as 68-75. The position of the $\mathrm{F}$ moorings are denoted by the letter ' $\mathrm{F}$ '. 
activity below $2000 \mathrm{~m}$. That section of the three profiles is excluded from the present analysis and treated separately. The reduced data set exhibits a variation of 140 in $\mathrm{N}^{2}$, from $1.2 \times 10^{-4} \mathrm{~s}^{-2}(100-200 \mathrm{db})$ to $8.6 \times 10^{-7} \mathrm{~s}^{-2}(2500-3000 \mathrm{db})$, resulting in a variation of 3.4 in $\mathrm{N}^{1 / 2}$. The variation in $\mathrm{S}_{\mathrm{z}}$ is minimal, from 2.23 to 3.56 times $\mathrm{S}_{\mathrm{zGM}}$, a factor of 1.6. The variability in the expressions involving frequency content was also small, a factor of 1.16 and 1.41 for the MM and HWF scalings, respectively. (See Table 3.2 for the bin averaged statistics.) Thus the difference in the $\mathrm{N}$ parameterizations of the models will not be masked by differences in their respective $(E,<\omega>)$ parameterizations.

The shear spectra (Figure 3.5) in this data subset qualitatively fit the canonical scenario of white until a wavenumber, $\mathrm{m}_{\mathrm{c}}$, which is independent of $\mathrm{N}$, and falling as $\mathrm{m}^{-1}$ thereafter. The data have been scaled in the vertical by multiplying $S_{z}$ by $N_{0}^{2} / N^{2}$ and in the horizontal by multiplying the wavenumber, $\mathrm{m}$, by $\mathrm{E}$. There is some tendency for the shear spectra from regions of low buoyancy frequency to lie above those from higher buoyancy frequencies in the $\mathrm{m}^{-1}$ region, but the scaling seems to do a good job in collapsing the data. Moreover, there was no evidence of vertical anisotropy or horizontal polarization. The internal wave characteristics of these data are not substantially differentiable from the GM prescription and thus we expect that this should be a good data set to test the GM based dynamical models.

We choose to examine the $(E, N,\langle\omega>)$ dependance in seven depth bins. Each depth bin represents a region of roughly constant buoyancy frequency. These depth bins appear in Table 3.3. Also 


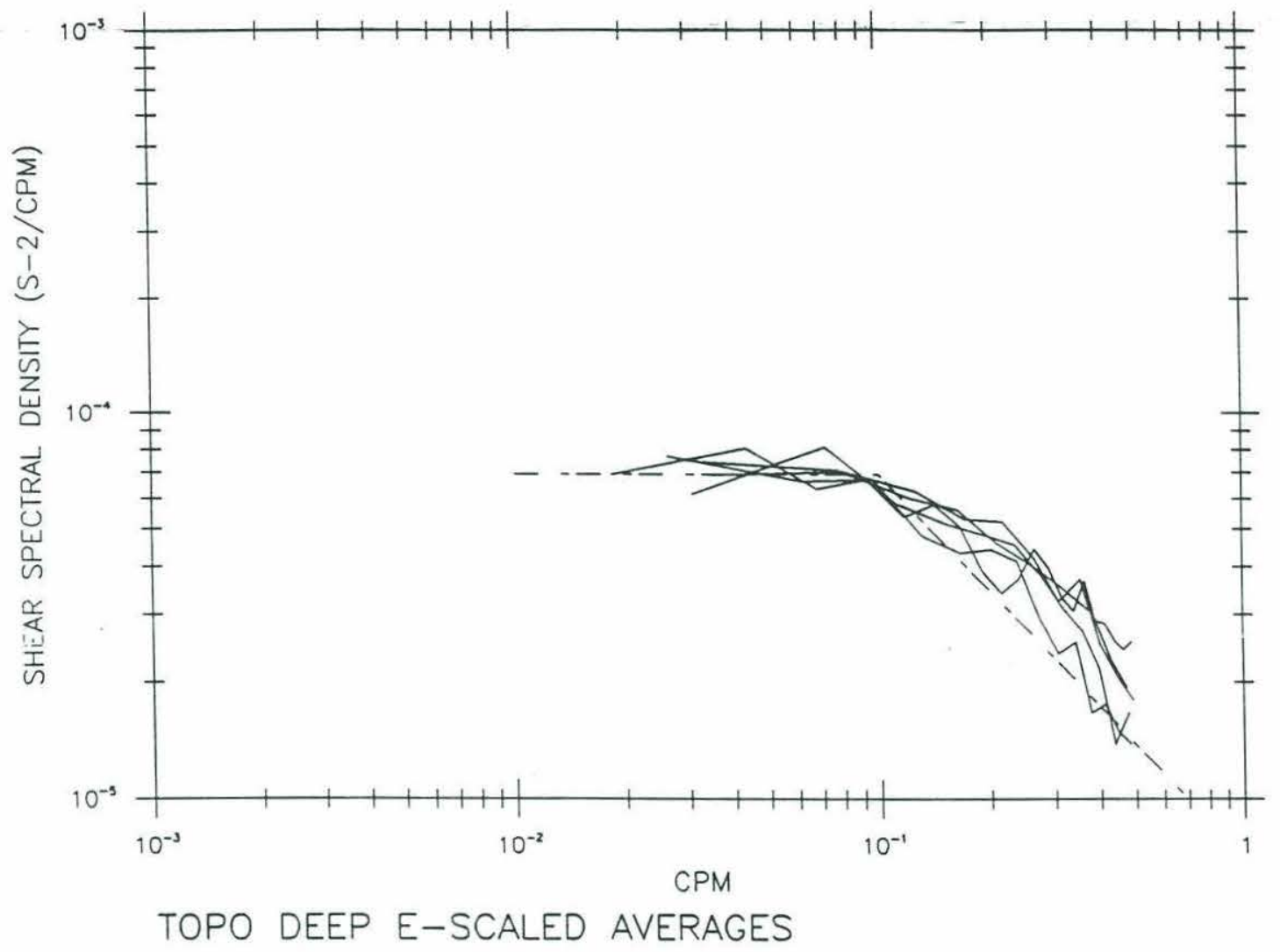

Figure 3.5: Shear spectra scaled in the vertical by $1 / \mathrm{EN}^{2}$ and in the horizontal by $\mathrm{E}$; and then divided by a factor of two. This scaling collapses the data quite effectively. The collapsed form agrees favorably with the canonical form in figure 3.1. The five lines represent averages from 200-400, 400-1000, 1000-1500, 15002000 , and $2000-3000 \mathrm{~m}$ for the reduced TOPO Deep data set. 
tabulated in Table 3.3 are the depth ranges over which the spectra are calculated. The spectra are overlapped as evenly as possible, e.g. a spectra nominally from 1500 to $1600 \mathrm{db}$ starts at $1486 \mathrm{db}$ and ends at $1614 \mathrm{db}$. There is a greater degree of overlap at the ends as bin 1 starts at $100 \mathrm{db}$ and bin 7 ends at $2978 \mathrm{db}$.

Table 3.2: Parameters for scaling the TOPO Deep data.

$\begin{array}{ccccccc}\text { Bin } & \mathrm{N}^{2} & \mathrm{~K}_{\rho} & \mathrm{E}_{1} & \mathrm{r}_{\omega} & \frac{<\omega>}{\mathrm{f}} & \left.\frac{\mathrm{N}}{\mathrm{f}}<\frac{\omega^{2}-\mathrm{f}^{2}}{\mathrm{~N}^{2}-\omega^{2}}\right)>^{1 / 2} \\ 1 & 1.20 \times 10^{-4} & .099 & 2.23 & 6.0 & 1.18 & .64 \\ 2 & 3.51 \times 10^{-5} & .069 & 2.25 & 6.6 & 1.16 & .60 \\ 3 & 1.05 \times 10^{-5} & .15 & 2.61 & 5.5 & 1.20 & .67 \\ 4 & 4.15 \times 10^{-6} & .097 & 2.81 & 4.6 & 1.24 & .74 \\ 5 & 2.53 \times 10^{-6} & .131 & 2.93 & 5.1 & 1.22 & .70 \\ 6 & 1.41 \times 10^{-6} & .098 & 2.58 & 4.8 & 1.23 & .73 \\ 7 & 8.59 \times 10^{-7} & .160 & 3.56 & 7.4 & 1.14 & .56\end{array}$

Table 3.3: Depth range and piece length of the TOPO Deep spectra.

$\begin{array}{cccccc}\text { Bin } & \begin{array}{c}\text { Nominal depth } \\ \text { range (in db) }\end{array} & \begin{array}{c}\text { piece } \\ \text { length }\end{array} & \begin{array}{l}\text { \# of } \\ \text { pieces }\end{array} & \begin{array}{c}\text { \# of } \\ \text { profiles }\end{array} & \begin{array}{c}\text { total } \\ \text { spec }\end{array} \\ 1 & 100-200 & 128 \mathrm{db} & 1 & 8 & 8 \\ 2 & 200-400 & 128 \mathrm{db} & 2 & 8 & 16 \\ 3 & 400-1000 & 128 \mathrm{db} & 6 & 8 & 48 \\ 4 & 1000-1500 & 128 \mathrm{db} & 5 & 8 & 40 \\ 5 & 1500-2000 & 128 \mathrm{db} & 5 & 8 & 40 \\ 6 & 2000-2500 & 256 \mathrm{db} & 2 & 5 & 10 \\ 7 & 2500-3000 & 256 \mathrm{db} & 2 & 5 & 10\end{array}$

The various parameters in the study are presented in Table 3.2. We choose to present $S_{z}$ in a nondimensional form by dividing $\mathrm{S}_{\mathrm{z}}$ by its GM specification, $1.38 \times 10^{-4} \mathrm{~s}^{-2} / \mathrm{cpm}$ (Munk 1981):

$$
\mathrm{E}_{1}=\mathrm{S}_{\mathrm{z}} / 1.38 \times 10^{-4}\left(\mathrm{~s}^{-2} / \mathrm{cpm}\right) \text {. }
$$

Dissipations are presented in the form of eddy diffusivities, 
$\mathrm{K}_{\rho}=.25<\varepsilon / \mathrm{N}^{2}>$. The ratio between the shear and strain spectra is followed by the derived estimates involving frequency content.

Before discussing the results of the scaling study, we wish to present profiles of $\mathrm{N}^{2}, \mathrm{E}_{1}$ and $\mathrm{K}_{\mathrm{\rho}}$ in Figure 3.6. The $\mathrm{N}^{2}$ values have been calculated by linear least square fits over $20 \mathrm{db}$ to pressure averaged profiles of temperature and salinity and are estimated at every half-decibar. The eddy diffusivities are averages over $100 \mathrm{~m}$. The $E_{1}$ values have been averaged over the depth range of the bins delineated in Table 3.3. In Figure 3.6, one finds that while $\mathrm{N}^{2}$ varies by over two orders of magnitude, there is little variation in $\mathrm{K}_{\rho}$ and $\mathrm{E}_{1}$. From this figure we anticipate the result of the scaling study that $\varepsilon \sim \mathrm{N}^{2}$ as opposed to $\varepsilon \sim \mathrm{N}^{+3 / 2}$.

The four scalings under consideration are:

M) $\varepsilon=\hat{\mathrm{E}} \frac{\sqrt{\mathrm{fN}}}{\pi}\left(\frac{\left(1+\ln \left(\frac{\mathrm{E}}{\mathrm{E}_{\mathrm{GM}}}\right)\right)}{4 \pi}\right)^{1 / 2} \exp \left\{-4 /\left(1+\ln \left(\frac{\mathrm{E}}{\mathrm{E}_{\mathrm{GM}}}\right)\right)\right\}$ (eq. 3.8)

$\mathrm{GH}) \varepsilon \sim \mathrm{EN}^{+3 / 2}$ (eq. 3.7)

$\mathrm{MM}) \varepsilon \sim \mathrm{N}^{2} \mathrm{E}^{2} \omega$ (eq. 3.3)

HWF) $\varepsilon \sim \mathrm{N}^{3} \mathrm{E}^{2}\left(\frac{\omega^{2}-\mathrm{f}^{2}}{\mathrm{~N}^{2}-\omega^{2}}\right)^{1 / 2} \quad$ (eq. 3.6)

We test the following expressions:

M) $\mathrm{K}_{\mathrm{p}}\left(\frac{\mathrm{N}}{\mathrm{N}_{\mathrm{o}}}\right)^{1 / 2} / \mathrm{qE} \mathrm{E}_{1}\left(1+\ln \left(\mathrm{E}_{1}\right)\right)^{1 / 2} \exp \left\{-4 /\left(1+\ln \left(\mathrm{E}_{1}\right)\right)\right\}$

GH) $\mathrm{K}_{\rho}\left(\frac{\mathrm{N}}{\mathrm{N}_{\mathrm{o}}}\right)^{1 / 2} / \mathrm{q}^{1 / 2} \mathrm{E}_{1}$

MM) $\mathrm{K}_{\rho} / \mathrm{q} \frac{<\omega>}{\mathrm{f}} \mathrm{E}_{1}^{2}$

HWF) $\left.\mathrm{K}_{\rho} / \mathrm{q} \frac{\mathrm{N}}{\mathrm{f}}<\frac{\omega^{2}-\mathrm{f}^{2}}{\mathrm{~N}^{2}-\omega^{2}}\right)>1 / 2 \mathrm{E}_{1}^{2}$ 


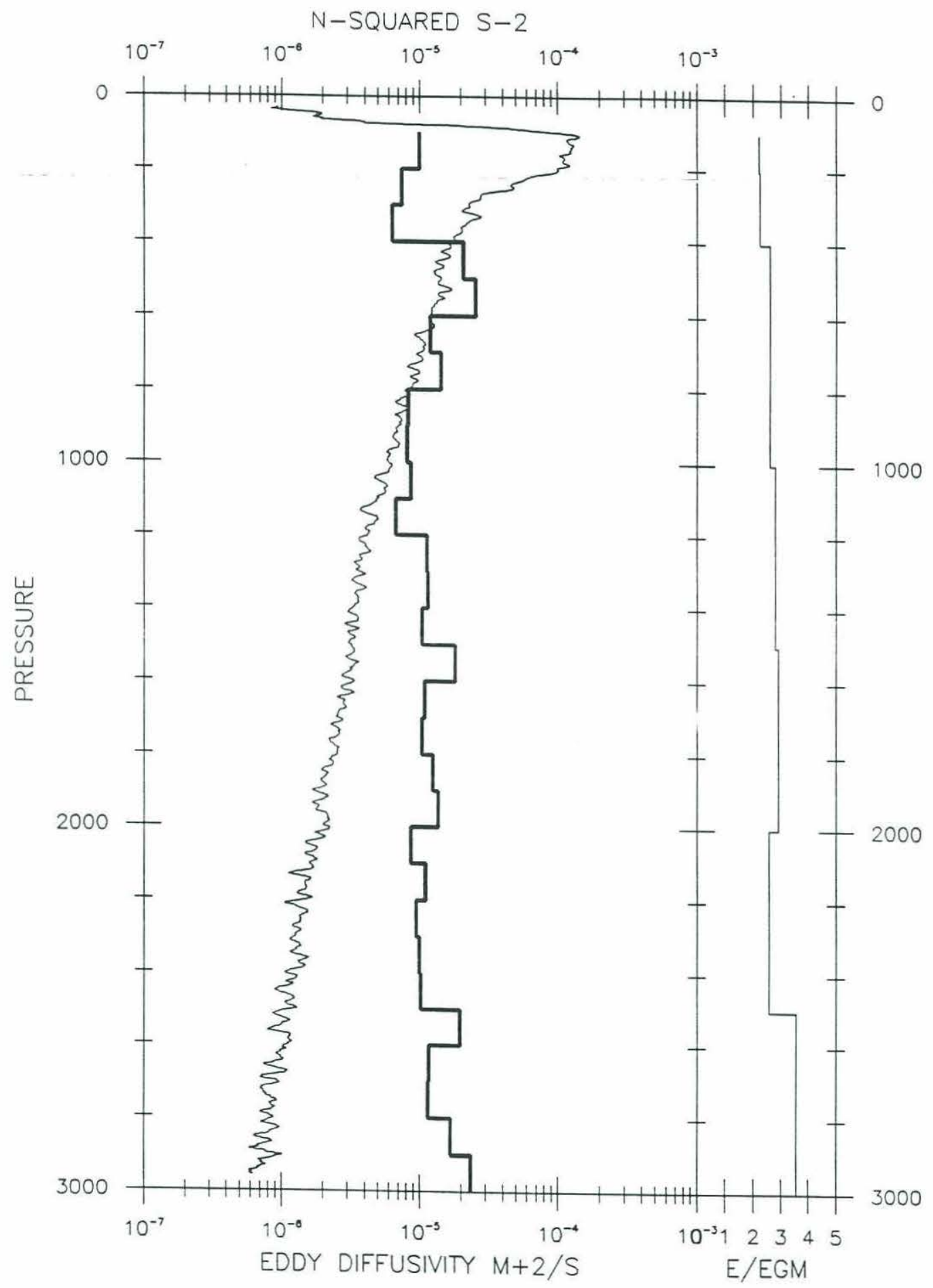

Figure 3.6: Profiles of $\mathrm{N}$-squared from average profiles of $\mathrm{T}$ and $\mathrm{S}$, $\mathrm{K}_{\rho}$, and $\mathrm{E}_{1}$. See text for details. 
The factor $\mathrm{q}$ is a correction for the assumption that the ratio between the kinetic and potential energies is constant in the scaling arguments. The ratio is actually a function of the frequency as discussed in the previous section. For the scaling of $\mathrm{MM}, \varepsilon \sim \mathrm{Dm}^{-1}$ $\mathrm{dE} / \mathrm{dm}$ such that $\mathrm{D} \sim \mathrm{S}_{\mathrm{z}} \omega \mathrm{m}^{3} / \mathrm{N}^{2}$. Since $\mathrm{dE} / \mathrm{dm}=\mathrm{m}^{-2}\left(\mathrm{~S}_{\mathrm{z}}+\mathrm{N}^{2} \mathrm{~F}_{\mathrm{z}}\right) / 2$, estimation of $\mathrm{dE} / \mathrm{dm}$ from $S_{z}$ will be a biased function of frequency. Hence we correct by the factor

$$
\mathrm{q}=\left(1+\frac{1}{\mathrm{r}_{\omega}}\right) \frac{3}{4} \text {. }
$$

The variability in the factor $\mathrm{q}$ is small for the data presently under consideration: we retain its use for consistency with the next section.

The scalings are presented versus buoyancy frequency in Figure 3.7. The data are presented such that the appropriate functional form should collapse the data onto a line parallel to the vertical axis. As stated previously, the greatest differences in the various parameterizations is the $\mathrm{N}$ scaling for these data. Hence an inappropriate functional form in $\mathrm{N}$ will be revealed as a trend. We find such a trend with the Munk and GH $\left(\varepsilon \sim \mathrm{N}^{+3 / 2}\right)$ parameterizations. No such trend is apparent in the MM and HWF plots.

The error bars in Figure 3.7 represent 95\% confidence intervals. The confidence intervals for $\mathrm{E}_{1}$ were calculated assuming chi-squared statistics for $S_{z}$ and two degrees of freedom for each (one-sided) Fourier coefficient. The confidence intervals for $\mathrm{K}_{\rho}$ were calculated via a boot strap method (Efron and Gong, 1983) and represent the third largest and smallest values of 100 sums formed by randomly sampling one-half of the data. These confidence intervals are not substantially different from the $95 \%$ confidence 


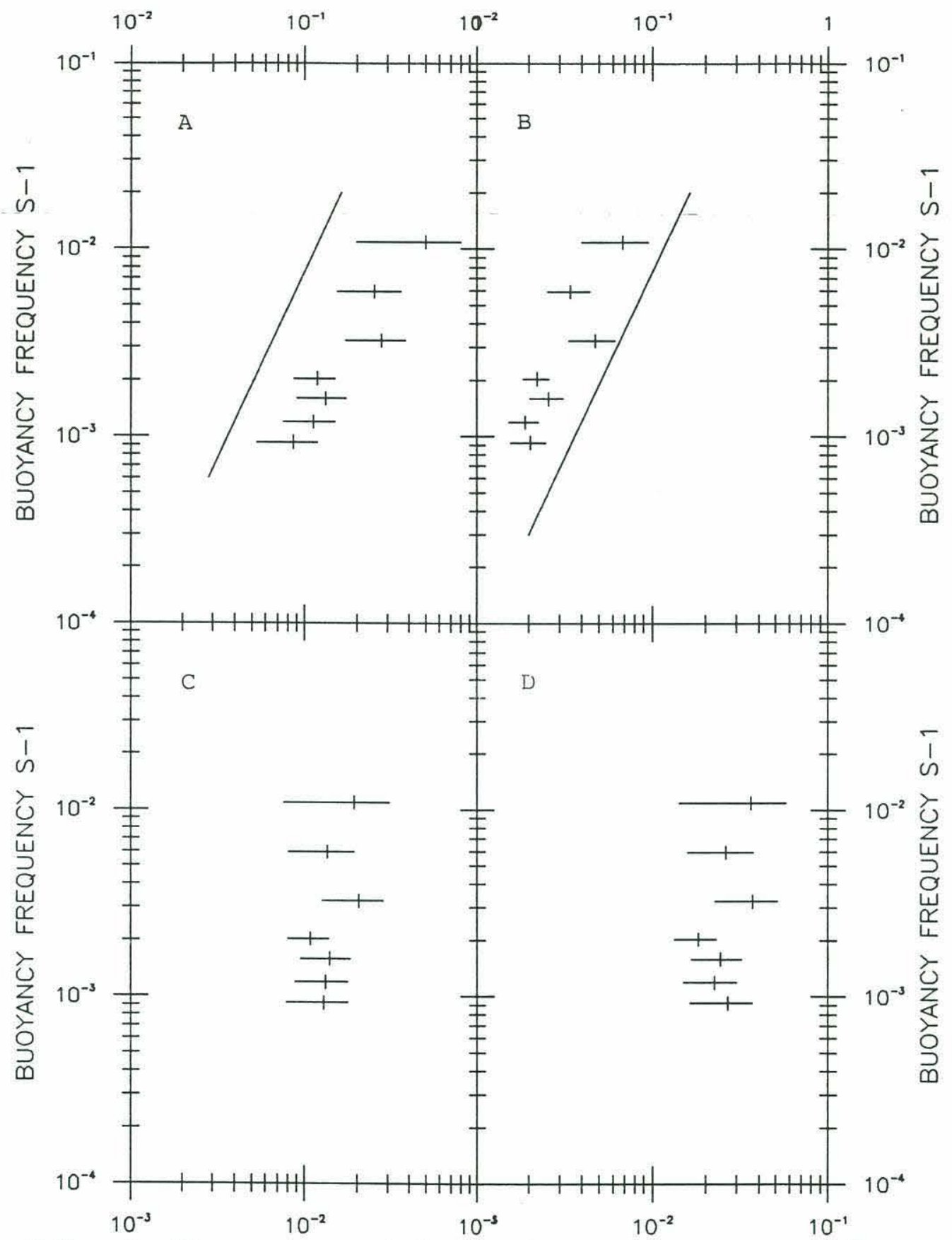

Figure 3.7: a) Bin averages of the Munk scaling, $\mathrm{K}_{\mathrm{p}}\left(\mathrm{N} / \mathrm{N}_{\mathrm{o}}\right)^{1 / 2} / \mathrm{qE}_{1}(1+$ $\left.\ln \left(E_{1}\right)\right)^{1 / 2} \exp \left\{-4 /\left(1+\ln \left(E_{1}\right)\right)\right\}$ versus $N$, where $K_{\rho}$ has units of $\mathrm{cm}^{2} / \mathrm{s}$. b) Bin averages of the Gargett and Holloway scaling $\mathrm{K}_{\mathrm{\rho}}\left(\mathrm{N} / \mathrm{N}_{0}\right)^{1 / 2} / \mathrm{q}^{1 / 2} \mathrm{E}_{1}$. c) Bin averages of the McComas and Muller scaling, $\mathrm{K}_{\rho} / \mathrm{q}(<\omega>/ \mathrm{f}) \mathrm{E}_{1}^{2}$. d) Bin averages of the Henyey et. al. scaling, $\mathrm{K}_{\mathrm{\rho}} / \mathrm{q}(\mathrm{N} / \mathrm{f})<\left(\left(\omega^{2}-\mathrm{f}^{2}\right) /\left(\mathrm{N}^{2}-\omega^{2}\right)\right)^{1 / 2}>\mathrm{E}_{1}^{2}$. The sloping line in a) and $\left.\mathrm{b}\right)$ represents an $\mathrm{N}^{1 / 2}$ dependence. The Munk and GH scaling reveal a distinct trend with $\mathrm{N}$ and appear to be inconsistent with the data. 
intervals based upon a log-normal distribution (Baker and Gibson, 1987). The total confidence intervals are calculated from the sum of the fractional errors in $E_{1}$ and $K_{\rho}$ for the $G H$ scaling and the sum of twice the fractional error in $E_{1}$ plus the fractional error in $K_{\rho}$ for the other three scalings. No error estimate is offered for the estimates derived from the ratio between the shear and the strain as we expect these two quantities to be functionally related and are thus uncertain about how to assess the error. We realize that geophysical data often do not meet the assumptions from which the statistical confidence intervals are calculated. However, we find it encouraging that the trends sighted in the GH and Munk scalings are sufficient to reveal confidence intervals which do not overlap.

We conclude that an $\varepsilon \sim \mathrm{EN}^{+3 / 2}$ or $\varepsilon \sim \mathrm{E}^{2} \mathrm{~N}^{+3 / 2}$ scaling is inappropriate for these data.

\section{$\underline{(\mathrm{E}, \underline{\omega}) \text { scaling }}$}

The $(\mathrm{E}, \omega)$ scaling of the dissipation parameterizations is examined by selecting portions of various data sets. We have chosen the TOPO deep profiles, including the three profiles below $2000 \mathrm{~m}$ which exhibited elevated E values; a set of 15 profiles from the flank of Fieberling Guyot in approximately $1500 \mathrm{~m}$ of water (TOPO_F, see Figure 3.4); and a set of 40 profiles from the center of a warm core ring (WRINCLE).

The TOPO_F profiles consist of two time series of 24 hours duration and a nominal sampling interval of three hours. The time series are separated by 13 days. The profiles were acquired within 100 meters of a position located between a set of three moorings (the 
F moorings). We choose to examine an interval between 700 and 1300 meters in the vertical. The heightened $\mathrm{E}$ values found in this depth interval are presumed to be due to the bottom reflection of propagating waves. Reflection of internal waves from a sloping bottom generally produces heightened shear levels as the incoming waves are preferentially reflected into higher vertical wavenumber outgoing waves. The greatest amplification of shear levels occurs when the group velocity vector parallels the bottom slope (Eriksen, 1985). The slope in the vicinity of these profiles is large, approximately $30^{\circ}$, and results in a critical frequency of $N / \sqrt{3}$. Hence higher than average frequency content is expected. The data are transformed as three overlapped 256 meter pieces (Table 3.5). Average buoyancy frequencies are between $2.0-3.0 \times 10^{-3} \mathrm{~s}^{-1}$ (Table 3.4). No statistically significant signatures of horizontal anisotropy or vertical inhomogeneity is apparent in the shear spectra.

Heightened energy levels are found in warm-core rings, presumably due to more efficient generation processes (e.g. Rubenstein and Roberts, 1986) and/or trapping of waves generated at the surface with frequencies below the planetary value for the inertial frequency (Kunze, 1985). The dominance of clockwise polarized motions over anti-clockwise is taken as denoting a preponderance of downward energy propagation (Leaman and Sanford, 1975) (Figure 3.8). We expect that the internal wave motions will be close to inertial in character: the largest ratios between the shear and strain for the data sets under consideration are found for the warm ring data. At the depths of interest (350$650 \mathrm{db}$ ), the relative vorticity is $5-15 \%$ of $\mathrm{f}$ ( $\mathrm{R}$. Schmitt, personal 


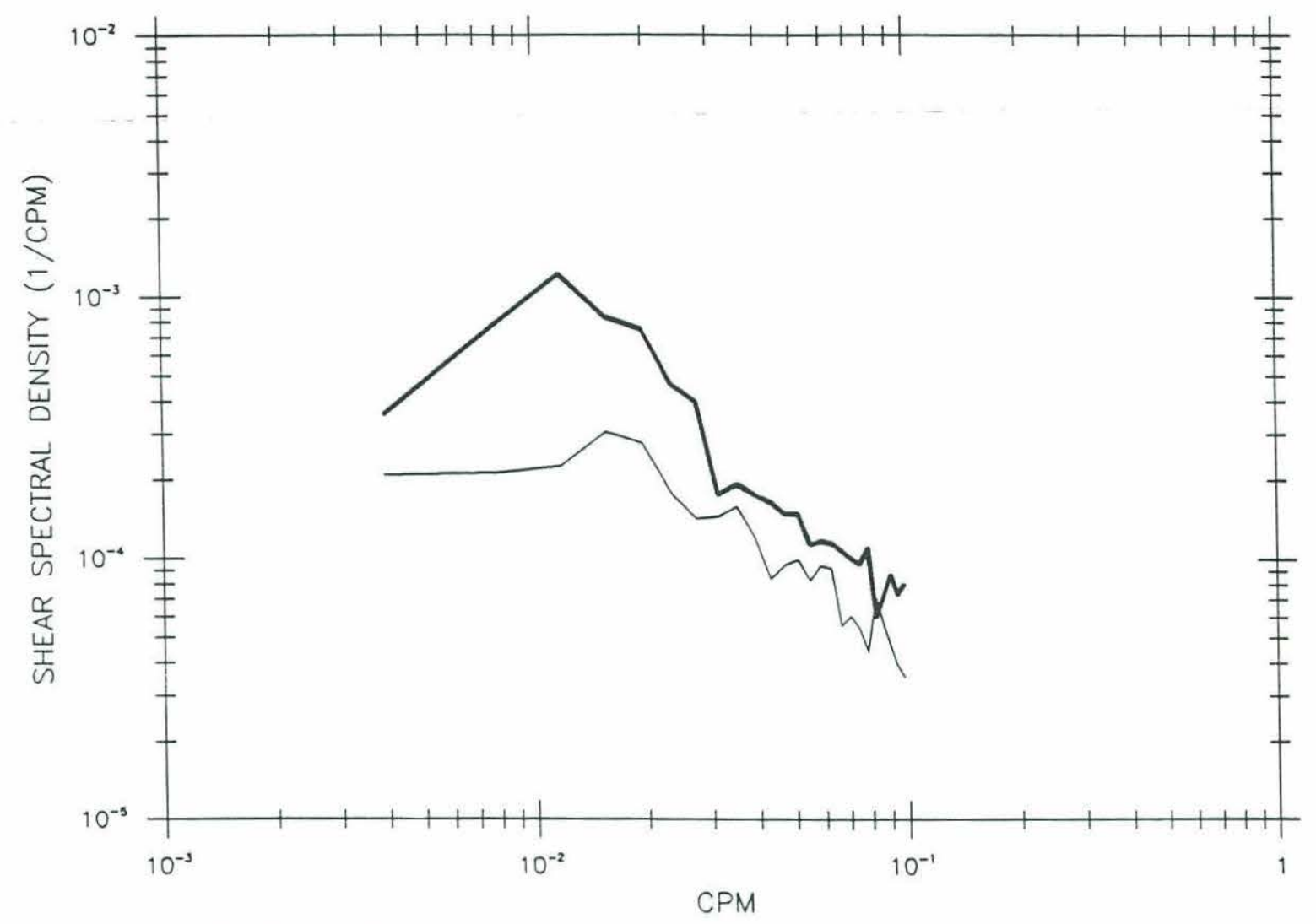

WRINCLE $372-628$ DB

Figure 3.8: $\mathrm{CW}$ (thick line) and $\mathrm{CCW}$ (thin line) shear spectra for the WRINCLE data. Data from 40 profiles between 372-628 meters have been averaged. The CW spectra dominate, denoting a preponderance of downward energy propagation. 
communication) It is assumed in these calculations that the relevant $\mathrm{f}$ is the planetary value $\left(40^{\circ} \mathrm{N}\right)$ and is not influenced by the background flow. One 256 meter spectra centered on 400-600 meters was obtained from each of the 40 profiles (Table 3.5). The average buoyancy frequency is $5.2 \times 10^{-3} \mathrm{~s}^{-1}$ (Table 3.4 ).

Table 3.4: Scaling parameters for the TOPO Deep profiles exhibiting heightened energy levels, the TOPO_F and WRINCLE data sets.

$\begin{array}{lcccccc}\text { Bin } & \mathrm{N}^{2} & \mathrm{~K}_{\rho} & \mathrm{E}_{1} & \mathrm{r}_{\omega} & \frac{<\omega>}{\mathrm{f}} & \frac{\mathrm{N}}{\mathrm{f}}\left\langle\frac{\omega^{2}-\mathrm{f}^{2}}{\mathrm{~N}^{2}-\omega^{2}}\right)^{1 / 2}> \\ 6^{\prime} & 1.41 \times 10^{-6} & .341 & 5.20 & 7.28 & 1.15 & .56 \\ 7^{\prime} & 8.59 \times 10^{-7} & 1.54 & 12.0 & 6.94 & 1.15 & .58 \\ \text { F1 } & 8.86 \times 10^{-6} & .862 & 5.22 & 4.74 & 1.24 & .73 \\ \text { F2 } & 6.11 \times 10^{-6} & .667 & 3.74 & 3.87 & 1.30 & .83 \\ \text { F3 } & 4.19 \times 10^{-6} & 1.24 & 3.69 & 2.30 & 1.59 & 1.24 \\ \text { W1 } & 2.63 \times 10^{-5} & .843 & 6.47 & 9.33 & 1.11 & .49 \\ \text { W2 } & 2.62 \times 10^{-5} & .682 & 8.81 & 17.0 & 1.06 & .35 \\ \text { W3 } & 2.62 \times 10^{-5} & 1.19 & 11.3 & 13.5 & 1.08 & .40 \\ \text { W4 } & 2.67 \times 10^{-5} & .763 & 6.38 & 7.69 & 1.14 & .55\end{array}$

Table 3.5: Depth range and piece length of spectra in Table 3.4.

$\begin{array}{cccccc}\text { Bin } & \begin{array}{c}\text { Nominal depth } \\ \text { range (in db) }\end{array} & \begin{array}{c}\text { piece } \\ \text { length }\end{array} & \begin{array}{c}\text { \# of } \\ \text { pieces }\end{array} & \begin{array}{c}\text { \# of } \\ \text { profiles }\end{array} & \begin{array}{c}\text { total } \\ \text { spec }\end{array} \\ 6^{\prime} & 2000-2500 & 256 \mathrm{db} & 2 & 3 & 6 \\ 7^{\prime} & 2500-3000 & 256 \mathrm{db} & 2 & 3 & 6 \\ & & & & & \\ \text { F1 } & 700-900 & 256 \mathrm{db} & 1 & 15 & 15 \\ \text { F2 } & 900-1100 & 256 \mathrm{db} & 1 & 15 & 15 \\ \text { F3 } & 1100-1300 & 256 \mathrm{db} & 1 & 15 & 15 \\ & & & & & \\ \text { W1 } & 400-600 & 256 \mathrm{db} & 1 & 10 & 10 \\ \text { W2 } & 400-600 & 256 \mathrm{db} & 1 & 10 & 10 \\ \text { W3 } & 400-600 & 256 \mathrm{db} & 1 & 10 & 10 \\ \text { W4 } & 400-600 & 256 \mathrm{db} & 1 & 10 & 10\end{array}$


Once the diffusivities have been scaled by $\mathrm{E}_{1}^{2}$, the remainder of the variability appears to be associated with the frequency content (Figure 3.9). This variability exceeds an order of magnitude, far beyond the factor of 2-3 represented by the confidence intervals. In Figure $3.9, \mathrm{~K}_{\rho} / \mathrm{E}_{1}^{2}$ is plotted versus $\mathrm{N}(\mathrm{a}), \mathrm{E}_{1}(\mathrm{~b})$, and $\mathrm{r}_{\omega}(\mathrm{c})$. The only consistent trend appears to be in $\mathrm{K}_{\rho} / \mathrm{E}_{1}^{2}$ versus $\mathrm{r}_{\omega}$, where $\mathrm{r}_{\omega}$ is related to the frequency content and the $\mathrm{PE} / \mathrm{KE}$ correction through equations $3.10,3.11$ and 3.12 .

In particular, note that bins $6,7,6^{\prime}$ and $7^{\prime}$ of the TOPO Deep data are indistinguishable under this scaling (Figure 3.9 a). The variability in $E_{1}$ far exceeds that of the frequency content for these data. Since the $95 \%$ confidence intervals for bins 6 ' and 7 are approximately a factor of 3 and 4, respectively (Figures 3.10 and 3.11 ), and the variability in the $\mathrm{E}_{1}$ values is a factor of two and three, respectively, we conclude that an $\varepsilon \sim \mathrm{EN}^{2}$ scaling is unlikely.

The MM and HWF parameterizations for these data are presented in Figures 3.10 and 3.11. Here we test the expressions: MM) $\left[.25 \varepsilon\left(\mathrm{E}_{\mathrm{GM}}, \mathrm{N}_{\mathrm{o}}\right) / \mathrm{N}_{\mathrm{o}}^{2}\right]\left(\frac{\langle\omega\rangle}{\mathrm{f}}\right)_{\mathrm{GM}} / \mathrm{q} \frac{\langle\omega\rangle}{\mathrm{f}} \mathrm{E}_{1}^{2},\left(\frac{\langle\omega\rangle}{\mathrm{f}}\right)_{\mathrm{GM}}=1.41$

HWF $)\left[.25 \varepsilon\left(\mathrm{E}_{\mathrm{GM}}, \mathrm{N}_{\mathrm{o}}\right) / \mathrm{N}_{\mathrm{o}}^{2}\right] / \mathrm{q} \frac{\mathrm{N}}{\mathrm{f}} \triangleleft\left(\frac{\omega^{2}-\mathrm{f}^{2}}{\mathrm{~N}^{2}-\omega^{2}}\right)^{1 / 2}>\mathrm{E}_{1}^{2}$

where $\varepsilon\left(\mathrm{E}_{\mathrm{GM}}, \mathrm{N}_{\mathrm{o}}\right)$ is calculated from eq.s 3.2 and 3.5, respectively, and the GM value of the frequency expression in the HWF parameterization is 1.00 .

The results are plotted as the observed diffusivity versus the model estimate. For this method of display, agreement between the model and data would be denoted by the points falling on a straight 


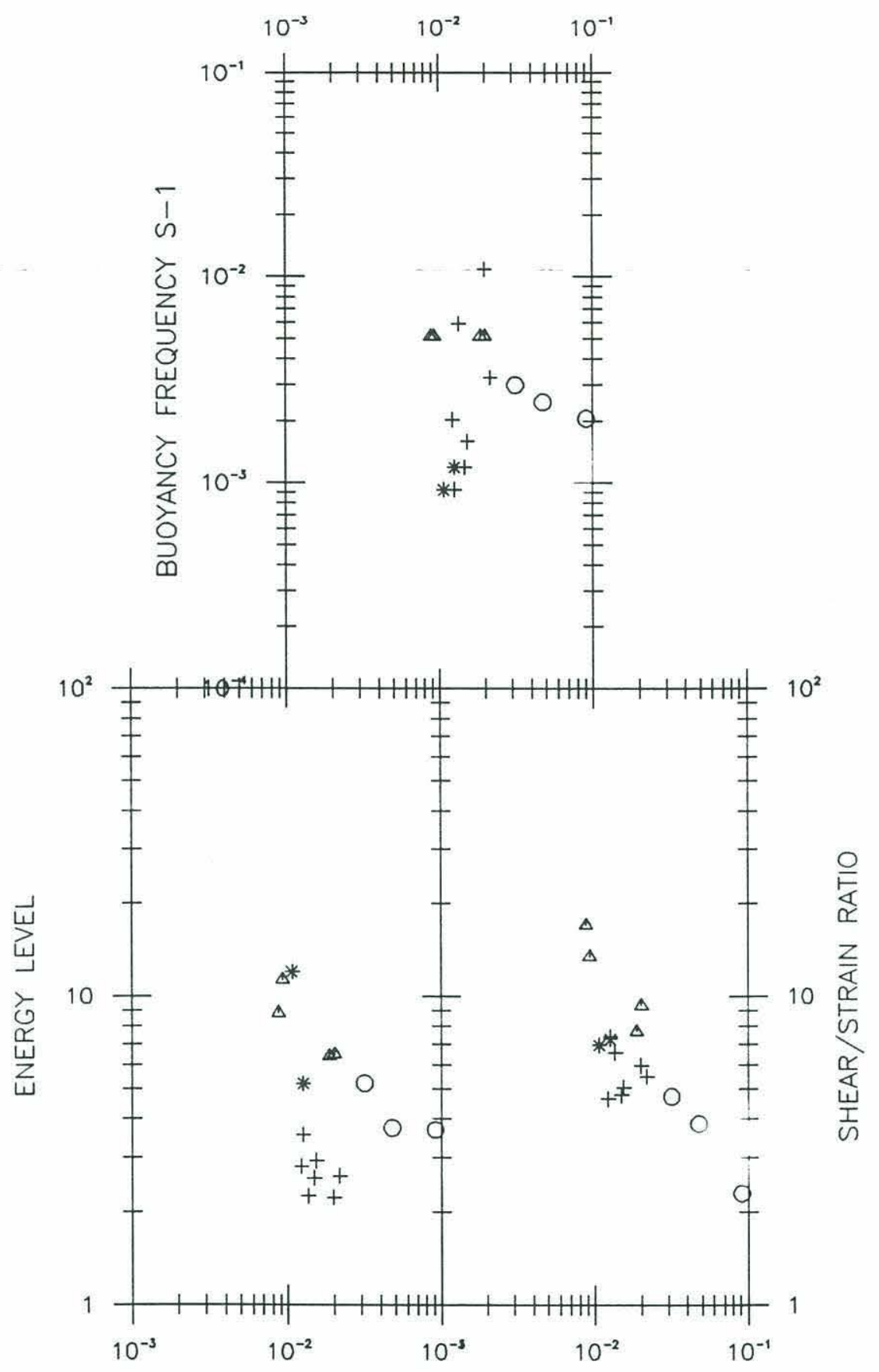

Figure 3.9: Bin averages of $\mathrm{K}_{\rho} / \mathrm{E}_{1}^{2}$ plotted versus a) $N$, b) $E_{1}$ and c) the shear/strain ratio $r_{\omega}$. Plus signs denote the TOPO Deep data, asterisks the TOPO Deep data exhibiting heightened energy levels below $2000 \mathrm{~m}$, circles the TOPO_F data, and triangles the WRINCLE data. The only consistent trend appears to be in $K_{\rho} / E_{1}^{2}$ versus $r_{\omega}$. 


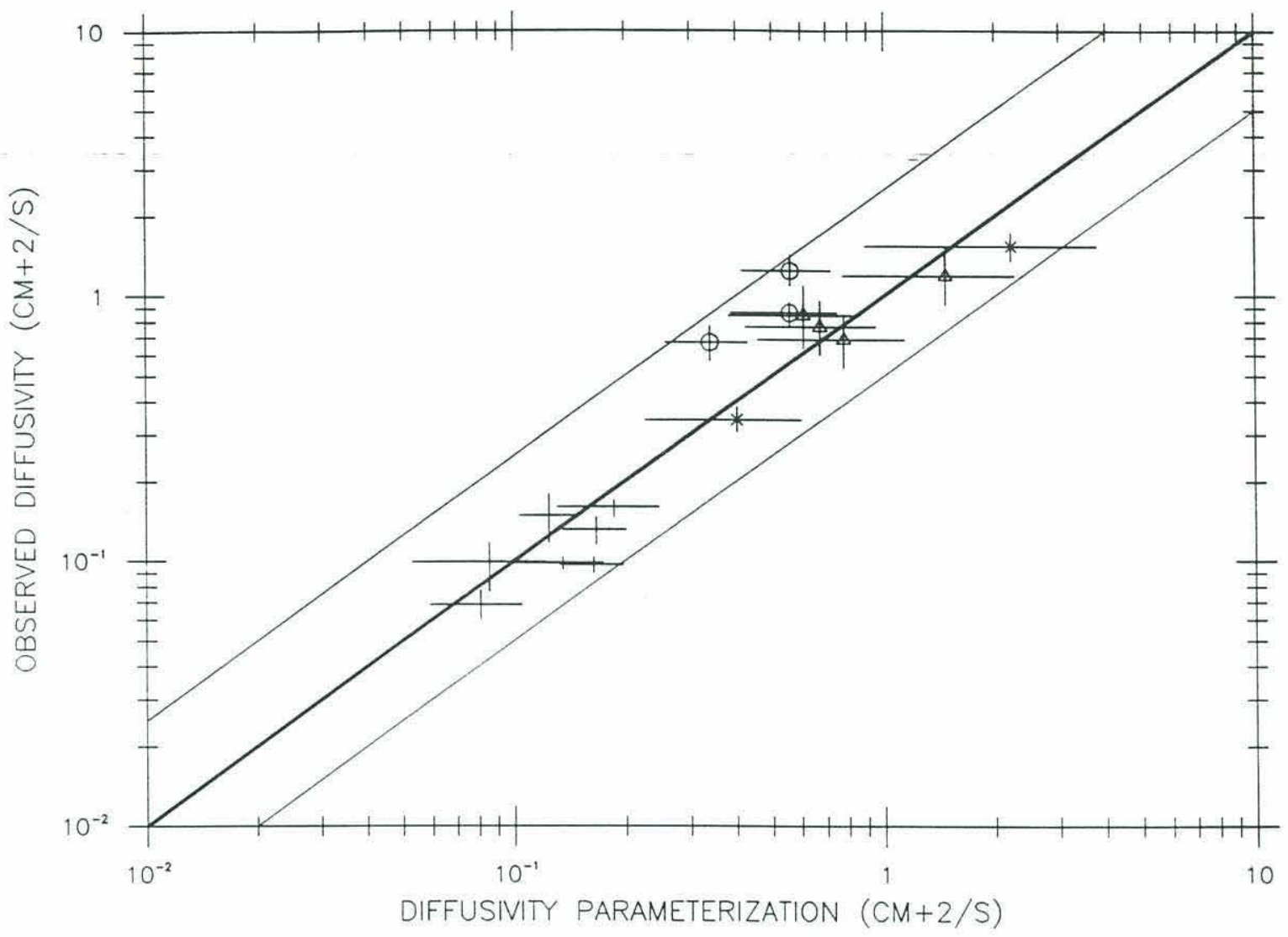

Figure 3.10: Bin averaged diffusivities, $\mathrm{K}_{\rho}$, plotted versus the Henyey et. al. parameterization, $\left.\left[.25 \varepsilon\left(\mathrm{E}_{\mathrm{GM}}, \mathrm{N}_{\mathrm{o}}\right) / \mathrm{N}_{\mathrm{o}}^{2}\right] / \mathrm{q}(\mathrm{N} / \mathrm{f})<\left(\left(\omega^{2}-\mathrm{f}^{2}\right) /\left(\mathrm{N}^{2}-\omega^{2}\right)\right)\right\rangle^{1 / 2}$ $\mathrm{E}_{1}^{2}$. The thick line denotes the model prediction, the thin lines bracket the outliers. The WRINCLE data are represented by triangles, the TOPO_F data by circles, and the TOPO Deep data by plus signs and asterisks. The crosses denote $95 \%$ confidence intervals. 


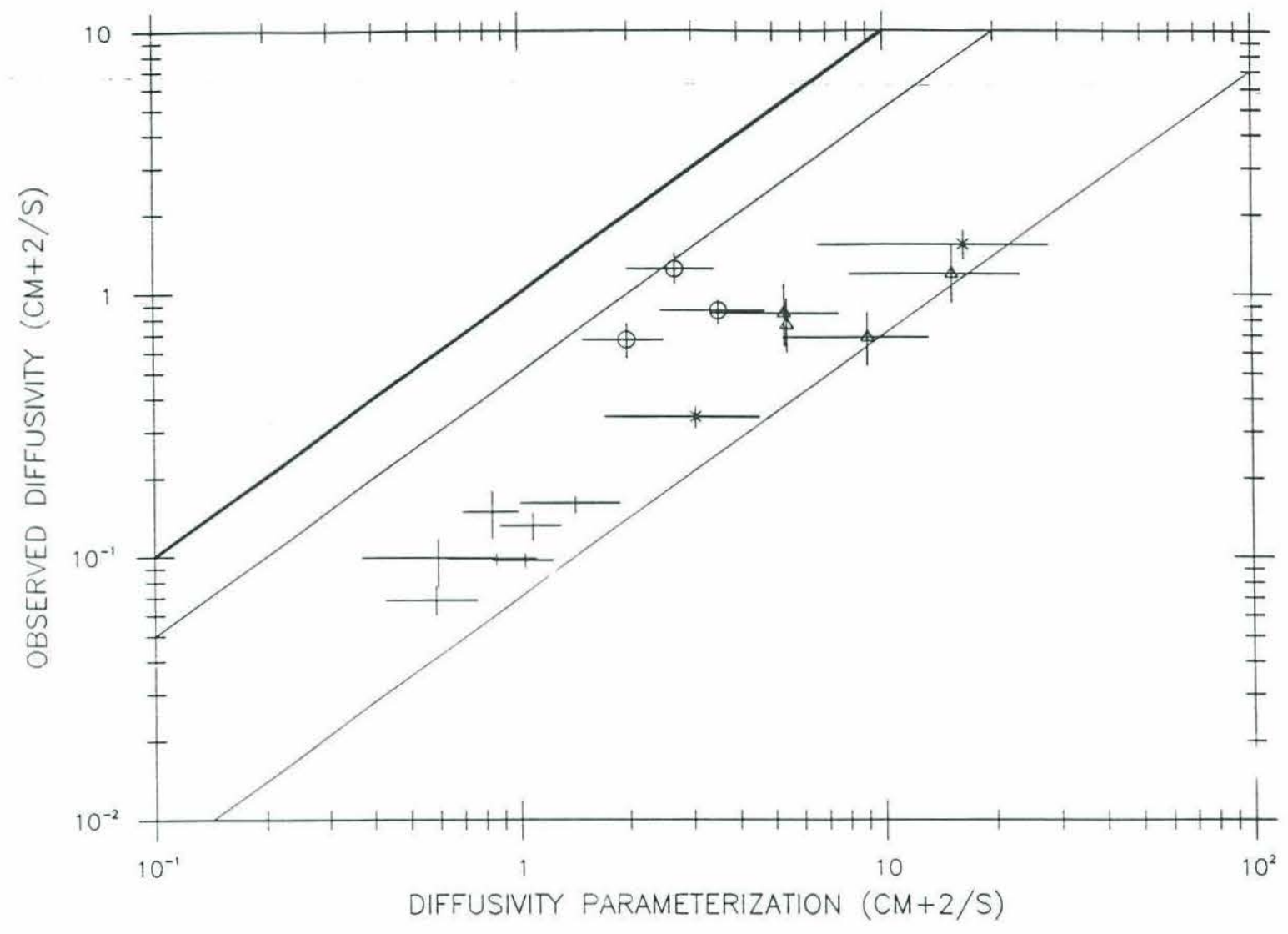

Figure 3.11: Bin averaged diffusivities, $\mathrm{K}_{\rho}$, plotted versus the McComas and Muller parameterization, $\left[.25 \varepsilon\left(\mathrm{E}_{\mathrm{GM}}, \mathrm{N}_{\mathrm{o}}\right) / \mathrm{N}_{\mathrm{o}}^{2}\right](<\omega>/ \mathrm{f})_{\mathrm{GM}} / \mathrm{q}(<\omega>/ \mathrm{f}) \mathrm{E}_{1}^{2}$. The thick line denotes the model prediction, the thin lines bracket the outliers. The WRINCLE data are represented by triangles, the TOPO_F data by circles, and the TOPO Deep data by plus signs and asterisks. The crosses denote $95 \%$ confidence intervals. 
line of slope one. Such lines are drawn in Figures 3.10 and 3.11. The thick line represents the model prediction and the thin lines bracket the outliers. The frequency content corrections succeed in collapsing the data further. The variability in the MM scaling becomes a factor of 5, and that remaining in the HWF scaling is slightly less, a factor of 4. We do not view this difference in variability, by itself, as being sufficient to differentiate between the two scalings. However, for the MM scaling, both high and low frequency data appear as outliers. Although the data characterized by higher than average internal wave frequency content are underestimated the most in the HWF parameterization, the low frequency data do not appear as outliers.

It appears that the HWF scaling captures the variability due to differences in the frequency content better than the MM scaling. However, descrepencies between the model assumptions and the data, which are addressed below, make such an assertion uncertain.

The models are differentiable in terms of their predictions for the magnitude of the diffusivity. The models differ in magnitude by a factor of five for a GM frequency spectrum. The model and data are not distinguishable from the HWF scaling, whereas the MM model overpredicts the data by a factor of five, on average.

\subsection{Discussion and Conclusions}

From averaged estimates of eddy diffusivities $\left(.25 \varepsilon / \mathrm{N}^{2}\right)$, shear and strain spectral densities at small $\left(\mathrm{m} \leq \mathrm{m}_{\mathrm{c}}\right)$ vertical wavenumbers, and buoyancy frequency, we examine the scalings of four models for the rate of dissipation of turbulent kinetic energy, $\varepsilon$. For a data set 
with little variation in the buoyancy scaled shear level (E) or frequency content (TOPO Deep), we find that the scaling of Gargett and Holloway (1984), $\varepsilon \sim \mathrm{EN}^{+3 / 2}$, and that of Munk (1981), $\varepsilon \sim \mathrm{E}^{2} \mathrm{~N}^{+3 / 2}$, to be inconsistent with an observed buoyancy scaling of approximately $\mathrm{N}^{2}$. Expansion of the parameter range in $\mathrm{E}$ at low $\mathrm{N}$ and roughly constant frequency content reveals a scaling which is not inconsistent with $\varepsilon \sim \mathrm{N}^{2} \mathrm{E}^{2}$, the scaling exhibited in the models of Henyey, Wright and Flatte (1986), and McComas and Muller (1981). Data from a warm core ring (dominated by near inertial frequencies) and from a region of steep topography (characterized by higher frequency motions) are analyzed in an attempt to expand the parameter range in frequency content. We find that the Henyey et al. scaling, $\varepsilon \sim\left(\omega^{2}-f^{2}\right)^{1 / 2}$, collapses the data more effectively than the scaling of McComas and Muller, $(\varepsilon \sim \omega)$. Moreover, the diffusivity estimates are in agreement with the model of Henyey et al. while the model of McComas and Muller over estimates the dissipation rate by a factor of five.

These results have immediate implications for oceanic mixing driven by internal wave motions. First, mixing, as denoted by an eddy diffusivity, is constant in the main thermocline. Moreover, the background rates for the GM spectral representation in Munk (1981) are small: $\mathrm{K}_{\rho}\left(\mathrm{E}_{\mathrm{GM}}\right)$ is approximately $.03 \mathrm{~cm}^{2} / \mathrm{s}$. Some variation in the observed value of $\mathrm{K}_{\rho}$ in the thermocline may be expected due to heightened energy levels associated with the interaction of internal waves with the mesoscale vorticity field or with seasonal fluctuations in surface forcing. Secondly, since the data are inconsistent with an inverse scaling of $\mathrm{K}_{\rho}$ with $\mathrm{N}$ at constant $\mathrm{E}$, some process or collection 
of processes must be responsible for heightened $\mathrm{E}$ values in the abyss if internal waves are responsible for producing the $\mathrm{O}(1-10$ $\mathrm{cm}^{2} / \mathrm{s}$ ) diffusivities generally inferred from hydrographic data. We view bottom reflection and/or bottom generation processes to be likely candidates: The average diffusivity in the deepest $100 \mathrm{~m}$ bin of the three TOPO Deep stations on the flank of Fieberling Guyot was $4 \mathrm{~cm}^{2} / \mathrm{s}$. These profiles were terminated approximately $400 \mathrm{~m}$ from the bottom.

The models of Henyey et al. and McComas and Muller associate $\varepsilon$ with the average energy flux through the vertical wavenumber spectrum at scales of 10 's to 100 's of meters under the assumption that the wavenumber and frequency spectra are statistically stationary. Results from these dynamical models indicate that the GM spectrum is stationary. The internal wave characteristics of the TOPO Deep data were not substantially differentiable from the GM prescription and the observed diffusivities were collapsed under the simple $(E, N)$ scaling $\left(\varepsilon \sim E^{2} N^{2}\right)$ predicted by those models for the GM spectrum

Data from a warm core ring and a region of steep topography were analyzed in order to examine the parameter dependence in internal wave fields which exhibited potentially non-stationary characteristics. The warm ring (WRINCLE) profiles were dominated by near-inertial frequencies, exhibited a large degree of vertical anisotropy and the presence of an anticyclonic background flow was noted. The frequency content of the TOPO_F data was higher than GM, presumabley associated with internal wave reflection processes. Moreover, the spectra of both data sets do not follow the canonical 
pattern of white until $\mathrm{m}_{\mathrm{c}}$, then as $\mathrm{m}^{-1}$ thereafter. In Figure 3.12 , the average $\mathrm{N}$-scaled shear spectra for the TOPO_F, and WRINCLE data sets have been shifted in the horizontal by a factor of E. Under the constant Richardson number scenario the spectra should be universal. However, these large $\mathrm{E}$ spectra are peaked at $\mathrm{m}_{\mathrm{c}}$ and do not fall off immediately thereafter. For the TOPO_F data set the high wavenumber slope seems to be appreciably gentler than -1 .

Of the possible non-equilibrium conditions in the internal wave field, the $(\mathrm{E}, \mathrm{N})$ scaled diffusivity estimates were most sensitive to deviations in wave field frequency content. The (E,N) scaled diffusivities revealed a consistent trend as a function of the frequency content. Application of a frequency based correction to the Henyey et al. model reduced more than an order of magnitude scatter in the scaled estimates to less than a factor of four.

These findings are consistent with those of Chapter Two in which data from an upper ocean front was examined. Inhomogeneity and anisotropy of the internal wave field were noted in that data set. The observed diffusivity estimates from the front were effectively collapsed under the (E,N) scaling of the model of Gregg (1989), which was in turn based upon the model of Henyey et al. The results of Chapter Two also suggested that the overall upwavenumber flux of energy through the internal spectrum is insensitive to deviations from the GM spectrum associated with anisotropy and inhomogeneity.

We note that there is a factor of two difference between Gregg's (1989) implementation of the Henyey et al. model and ours. Gregg surmised a scaling of, 


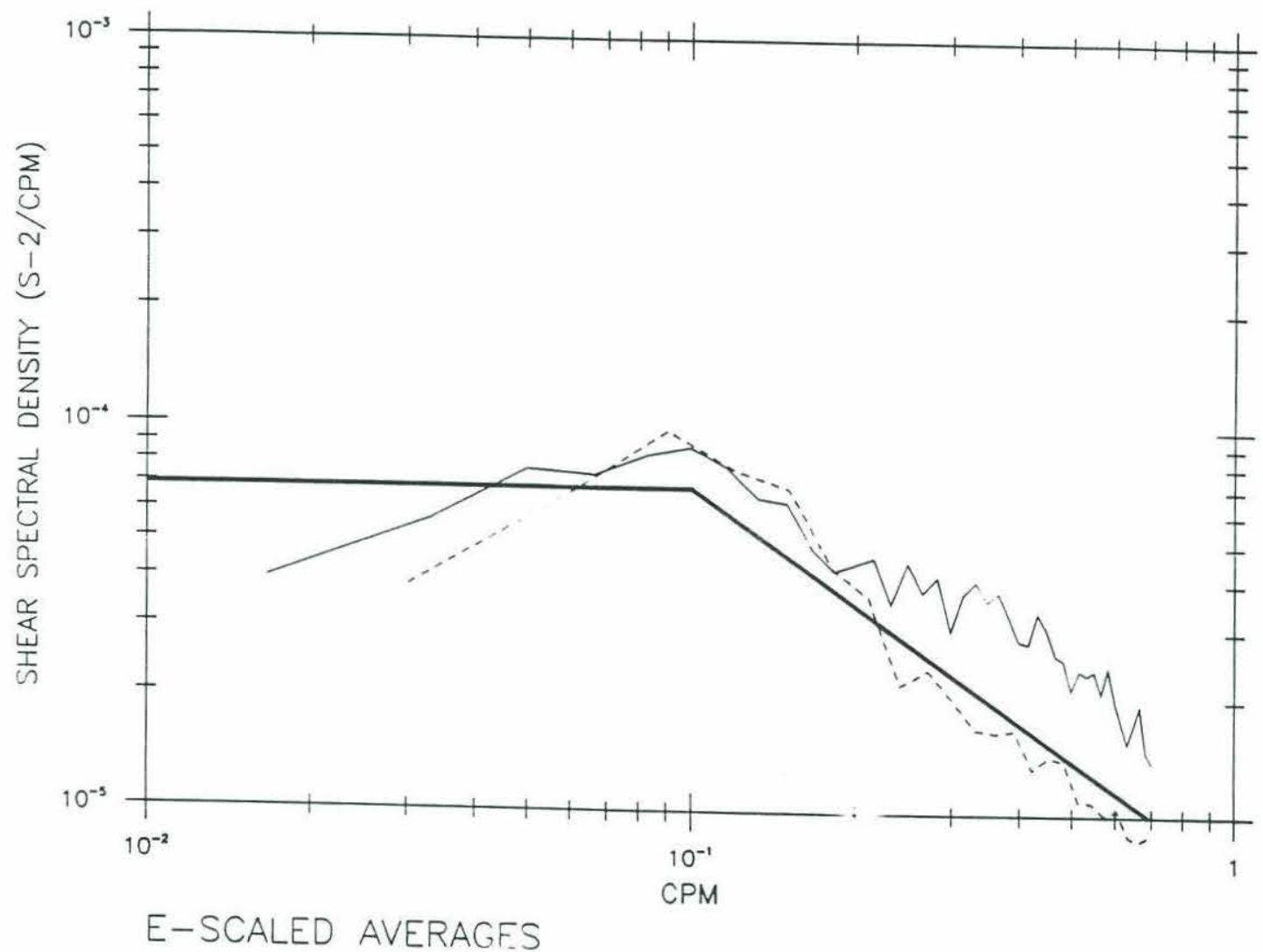

Figure 3.12: Shear spectra scaled in the vertical by $1 / \mathrm{EN}^{2}$ and in the horizontal by $\mathrm{E}$; and then divided by a factor of two. The collapsed form tends to be peaked about $\mathrm{m}_{\mathrm{c}}$ with respect to the canonical form in figure 3.1 and the TOPO Deep data in figure 3.5. The thin line represents the TOPO_F data, the dashed line the WRINCLE data, and the thick line the canonical form. The high wavenumber slopes for the TOPO_F data appear to be appreciabley gentler than -1 . 


$$
\varepsilon_{\mathrm{IW}}=7 \times 10^{-10}\left[\frac{\mathrm{N}}{\mathrm{N}_{\mathrm{o}}}\right]^{2}\left[\frac{\mathrm{E}}{\mathrm{E}_{\mathrm{GM}}}\right]^{2} \mathrm{~W} / \mathrm{kg} \text { at } 34^{\circ} \mathrm{N},
$$

which is twice the estimate of Henyey et al. at $34^{\circ} \mathrm{N}$ (eq. 3.5) and $\mathrm{N}=3 \mathrm{cph}$. The factor of two difference appears to be due to the use of GM '76 to reference $<S^{4}>$ estimates in Gregg (1989). There is a $\pi / 2$ difference between GM '76 and Munk '81 values for E (Gregg and Kunze, 1991), with GM '76 being the larger. Since HWF is based upon Munk '81, a consistent estimate of $\left\langle\mathrm{S}^{4}>/ 2\left(<\mathrm{S}^{2}\right\rangle_{\mathrm{GM}}\right)^{2}$ would provide $\mathrm{E}^{2}$ values $\pi^{2} / 4$ times larger and Gregg's $\varepsilon_{\text {IW }}$ parameterization would be consistent in magnitude with that magnitude of HWF for a GM internal wave field.

Gargett (1990) criticized the study of Gregg (1989) as suffering from a biased estimate of $\mathrm{E}$ at high energy levels (Section 3.3). The implication of such a criticism is that Gregg's warm core ring data (RING 82I) and PATCHEX data would not be collapsed under an $\mathrm{E}^{2} \mathrm{~N}^{2}$ scaling if an unbiased estimator for $\mathrm{E}$ was employed. We suspect that Gregg's RING 82I data was dominated by near inertial motions. From the present study we expect that such a frequency content will lead to lower than expected dissipations at a given energy level (eq. 3.6). It is our conjecture that the reason Gregg was able to collapse his PATCHEX and RING 82I data sets under the $\mathrm{E}^{2} \mathrm{~N}^{2}$ scaling of equation 3.13 was a cancellation between the bias errors in $\mathrm{E}$ and the lower than expected dissipations associated with lower than average frequency content. Gregg's DRIFTER data exhibited lower than expected levels of dissipation for a given energy level in a region dominated by a near-inertial feature at approximately $170 \mathrm{db}$ (Figure 3.13). The energy levels were not so large as the RING 82I 


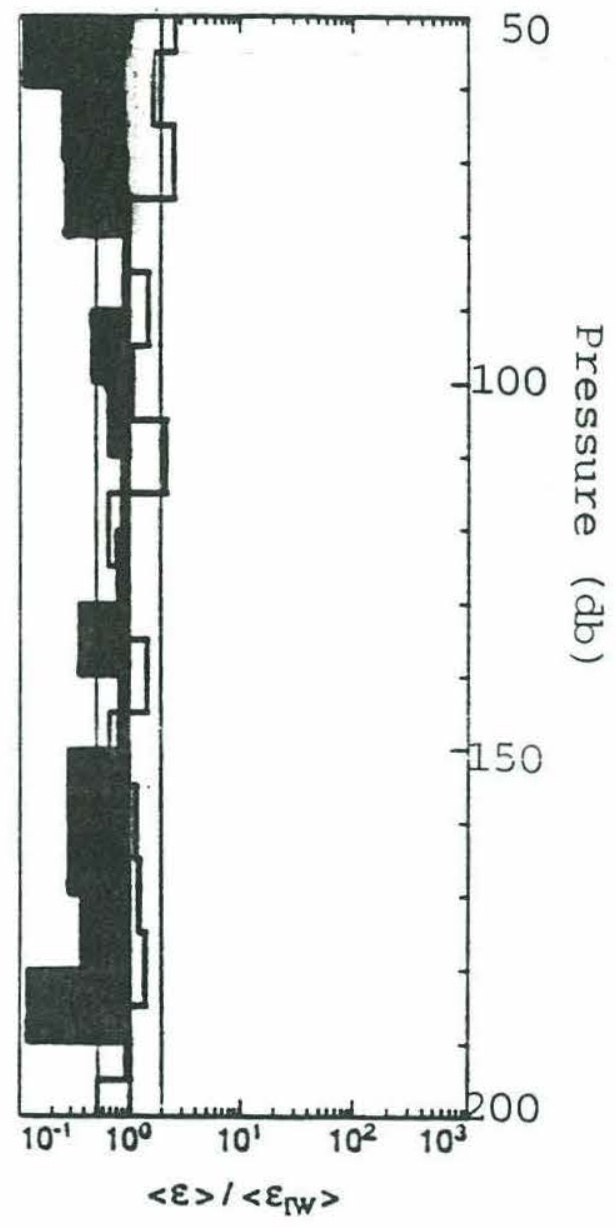

Figure 3.13: Drifter data from Gregg (1989). The DRIFTER curves have been colored in about $\langle\varepsilon\rangle \mid\left\langle\varepsilon_{\mathrm{iw}}\right\rangle=1$. The thin lines represent a factor of two about these values. There appears to be substantial disagreement between the DRIFTER data and Gregg's internal wave scaling in the depth interval $150-200 \mathrm{db}$. The remaining line represents the PATCHEX data set. 
values and thus suffer less bias problems in the estimation of $\mathrm{E}$.

Such a disagreement is consistent with our finding of lower than average frequencies leading to lower than expected dissipations at given energy levels. In this case the lower than expected dissipations were not cancelled by bias errors in E.

Finally, we note the appearance of the $\cosh ^{-1}(\mathrm{~N} / \mathrm{f})$ in the Henyey et al. scaling for an internal wave field of GM frequency content (eq. 3.5). The term $\cosh ^{-1}(\mathrm{~N} / \mathrm{f})$ is an increasing function of $\mathrm{N}$ and actually serves to increase the difference in the $\mathrm{N}$ scaling between $\mathrm{N}^{2} \cosh ^{-1}(\mathrm{~N} / \mathrm{f})$ and $\mathrm{N}^{3 / 2}$. Although the variability of $\cosh ^{-1}(\mathrm{~N} / \mathrm{f})$ is a factor of approximately 2 for the TOPO Deep data set which are collapsed better by its inclusion, we chose not to implement this term. Our reason for doing so is that this term arises after integrating the product of the horizontal wavenumber, $\mathrm{m}\left(\left(\omega^{2}-\mathrm{f}^{2}\right) /\left(\mathrm{N}^{2}-\omega^{2}\right)\right)^{1 / 2}$, and the inertial peak in the GM spectrum, $\left(\omega^{2}\right.$ $\left.f^{2}\right)^{-1 / 2}$, over all internal wave frequencies ( $f$ to $N$ ). The TOPO_Deep frequencies are lower than GM when estimated from eq. 3.10 and we suspect that the difference between the resulting functional form of the Henyey et al. model (i.e. $\mathrm{N}^{2} \cosh ^{-1}$ ) and $\mathrm{N}^{2}$ is sensitive to this cancellation. 


\title{
CHAPTER FOUR
}

\author{
A Richardson Number Model of Mixing \\ and \\ Statistics of the Richardson Number
}

\subsection{Introduction}

This chapter examines the parameterization of the rate of dissipation of turbulent kinetic energy in terms of Richardson number. We consider the Richardson number based model of Kunze, Williams and Briscoe (1990) in which large dissipation rates are associated with low Richardson number $\left(\mathrm{Ri}=\mathrm{N}^{2} / \mathrm{S}^{2}\right)$ events. The primary emphasis of this chapter is to examine the Kunze et al. model for its effectiveness as a turbulence parameterization scheme and to determine the scaling dependance of the model on the internal wave energy level, frequency content and background stratification rate. The microstructure measurements required to quantify turbulent mixing are not routine: they require specialized instrumentation with high spatial resolution and low vibrational characteristics. The parameterization attempt is designed to diagnose mixing in terms of larger scale, more easily (routinely) measured quantities. A successful parameterization would ideally result in the routine estimation of mixing. Once it is shown that the parameterization is effective in predicting the dissipation in a wide variety of environments, its scaling can be used to predict global patterns of dissipation. For example, given the apparent universality of the 
Garrett and Munk spectrum and knowledge of the climatological variability of the internal wave field (Wunsch and Webb, 1979), the mixing model could be used to predict the level of mixing in the bulk of the oceans.

A second goal is to elucidate the dynamics which controls the finescale shear spectral shape and level for scales smaller than $\mathrm{m}_{\mathrm{c}}^{-1}$, where $\mathrm{m}_{\mathrm{c}}$ is the cutoff wavenumber in the vertical spectrum (nominally $.1 \mathrm{cpm})$. In Chapter Three the parameterization of dissipation in the context of a number of wave/wave interaction models addressing vertical scales larger than $\mathrm{m}_{\mathrm{c}}^{-1}$ was examined. Those wave/wave interaction models are formally invalid at vertical scales smaller than $\mathrm{m}_{\mathrm{c}}^{-1}$ (Holloway, 1982; Henyey, Wright and Flatte, 1986) and cannot address the dynamics which control the flux of energy to higher wavenumbers in the transition region between $\mathrm{m}_{\mathrm{c}}^{-1}$ and turbulent scales (approximately 2-100 cpm). In this study, which is by necessity kinematical in nature, the mixing model of Kunze et al. is examined for vertical scales smaller than $\mathrm{m}_{\mathrm{c}}^{-1}$ to assess whether the mechanism of shear instability can provide an appropriate closure scheme.

Chapter Four consists of four major sections. The first (4.2) presents qualitative evidence for a Richardson number based mixing model and attempts to place such models within the context of more complex models which address the upwavenumber flux of energy in the internal wave field. In Section 4.3 the model of Kunze, Williams and Briscoe (1990) is applied to a number of data sets exhibiting a broad range of energy levels (E), background buoyancy frequencies $(\mathrm{N})$, and internal wave frequency content $(\langle\omega\rangle)$. Section 4.4 examines the statistics of the Richardson number and the resulting $(\mathrm{E}, \mathrm{N},<\omega>)$ 
scaling of the Kunze et al. model. Dynamical aspects of the problem are addressed in Section 4.5. Findings are summarized in Section 4.6

4.2 Qualitative evidence for the shear instability mechanism

A canonical internal wave shear spectrum $\left(\mathrm{S}_{\mathrm{Z}}\right)$ as a function of vertical wavenumber $(\mathrm{m})$ is depicted in Figure 4.1. Unless so noted, wavenumbers and frequencies have units of cycles per meter and second, respectively. The shear spectral shape follows the GM (Munk, 1981) spectrum at low wavenumber (approximately white) and the magnitude obeys WKB scaling $\left(\mathrm{S}_{\mathrm{Z}} \sim \mathrm{N}^{2}\right)$. The shear spectrum then falls as approximately $\mathrm{m}^{-1}$ for vertical wavenumbers larger then some cutoff, $\mathrm{m}_{\mathrm{c}}$. From an observational standpoint (Gargett et al., 1981; Duda and Cox, 1989; Gregg et al., 1991; Figure 3.5), there is evidence that the cutoff wavenumber $\mathrm{m}_{\mathrm{c}}$ is independent of $\mathrm{N}$ (i.e. does not WKB scale) and varies inversely with the average shear spectral density of wavenumbers $\mathrm{m}<\mathrm{m}_{\mathrm{c}}$, although both Duda and Cox (1989) and Gregg et al. (1991) point to quantitative exceptions. Researchers (Gargett et al., 1981; Munk, 1981) have suggested that the cuttoff wavenumber occurs at a universal value of the mean square Richardson number, $\mathrm{N}^{2} /<\mathrm{S}^{2}>$ (the universal Richardson number hypothesis, Gargett (1990)). In this study the transition from white to $\mathrm{m}^{-1}$ at a vertical wavenumber where

$$
\int_{0}^{\mathrm{m}_{\mathrm{c}}} \mathrm{S}_{\mathrm{z}} \mathrm{dm}=\mathrm{N}^{2} / 2
$$




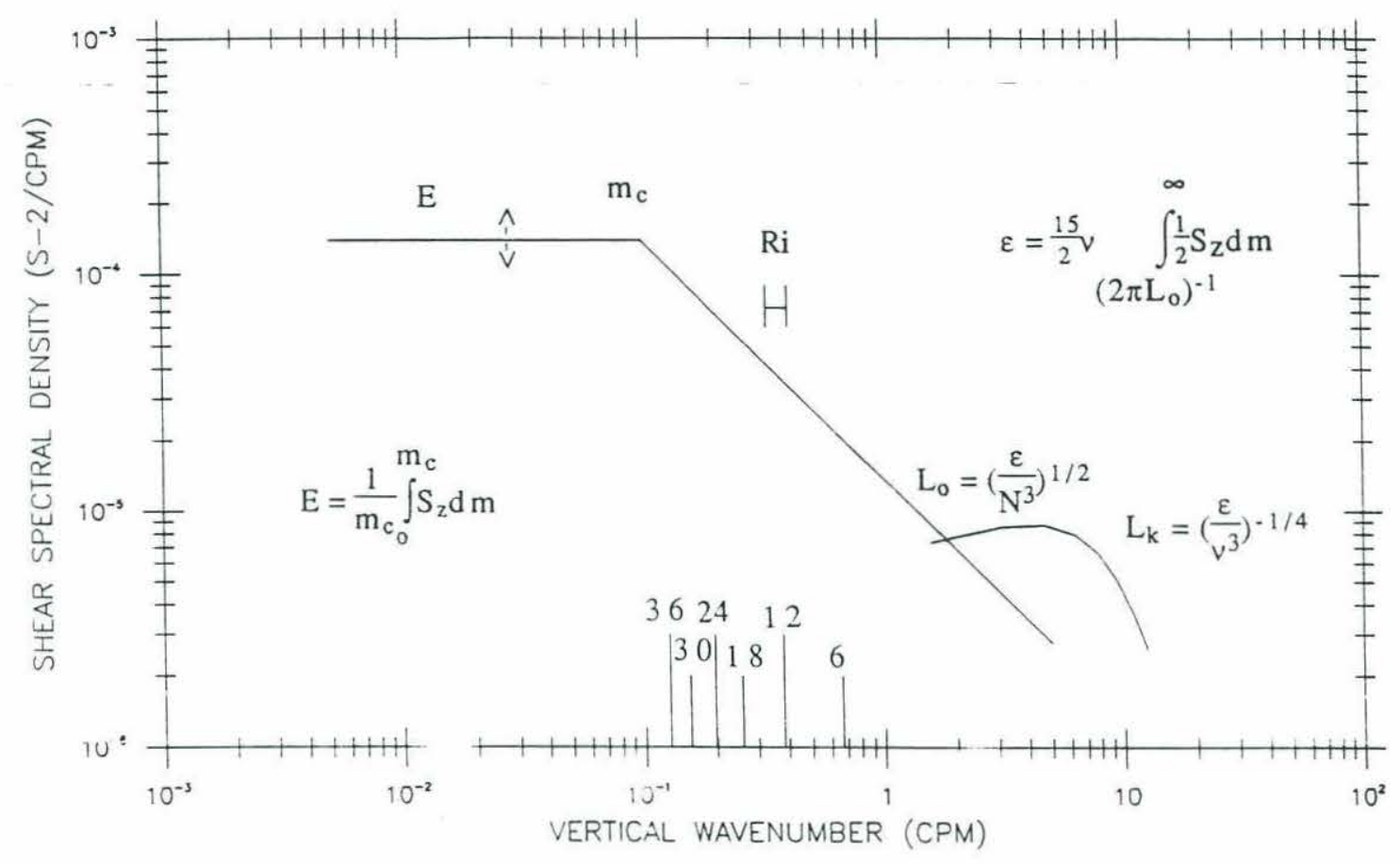

Figure 4.1: The canonical vertical wavenumber shear spectrum is white until $\mathrm{m}_{\mathrm{c}}$, decreases as $\mathrm{m}^{-1}$ until a scale $\left(2 \pi \mathrm{L}_{\mathrm{o}}\right)^{-1}$ and then terminates in a 'microstructure bump'. The low wavenumber regime is associated with weakly non-linear wave/wave interactions, the transition region $\mathrm{m}_{\mathrm{c}} \leq \mathrm{m} \leq\left(2 \pi \mathrm{L}_{\mathrm{o}}\right)^{-1}$ with strongly interacting and/or actively dissipating internal waves, and the high wavenumber regime with three-dimensional turbulence. See the text for a more detailed discussion of the length scales and symbols in the figure. 
is extensively exploited. This results in, approximately, $\mathrm{S}_{\mathrm{z}} \mathrm{m}_{\mathrm{c}}=\mathrm{N}^{2} / 2$, or $\mathrm{Em}_{\mathrm{c}}=$ constant (the universal Richardson number criterion, e.g. Gargett (1990)). In that which follows the nondimensional GM energy density level, E, is defined as the average shear spectral density normalized by $1 / \mathrm{N}^{2}$ for wavenumbers less than $\mathrm{m}_{\mathrm{c}}$. The shear spectral density rises at high wavenumbers $\left(\mathrm{m} \sim\left(2 \pi \mathrm{L}_{0}\right)^{-1}\right)$ and then falls at a scale $m \sim\left(2 \pi L_{k}\right)^{-1}$. The changes in the slope of the canonical shear spectrum presumably denote changes in the dynamics governing the motion.

The shape of the vertical wavenumber spectrum at high wavenumbers is attributable to turbulence. The scale $\mathrm{L}_{0}$, termed the 'Osmidov' length, is the length scale at which inertial forces (related to the dissipation rate, $\varepsilon$ ) are of the same order as buoyancy forces $(\mathrm{N}): \mathrm{L}_{\mathrm{o}}=\left(\varepsilon / \mathrm{N}^{3}\right)^{1 / 2}$. The Osmidov length can be related to the rms displacement in an overturn $\left(\mathrm{L}_{\mathrm{t}}\right.$, the Thorpe scale) by $\mathrm{L}_{\mathrm{o}} \sim .8 \mathrm{~L}_{\mathrm{t}}$ (Dillon, 1982). The maximum height of overturning events appears to be limited by $2 \pi \mathrm{L}_{0}$ (Gregg et al., 1991). The spectral density decreases past $\mathrm{L}_{\mathrm{k}}^{-1}$ (the Kolmogorov scale) due to viscous effects $\left(\mathrm{L}_{\mathrm{k}}=\left(\varepsilon / v^{3}\right)^{-1 / 4}\right)$. The region between overturning scales and those at which kinetic energy is dissipated by viscosity is generally interpreted in terms of universal, isotropic turbulence (Gregg (1987), Yamazaki (1990)), although this is not always the case (Gibson, 1986). If the dissipation is sufficiently weak, overturning scales will be significantly affected by viscosity and restratification is to be expected (e.g. Itsweire, 1986). The requirement for an effective buoyancy flux within a turbulent region, $\varepsilon>15 \mathrm{vN}^{2}$ (Itsweire et al. 1983), can be expressed in terms of a scale separation: $\mathrm{L}_{\mathrm{o}}>8 \mathrm{~L}_{\mathrm{k}}$. This criterion is not directly 
applicable to the above spectrum as turbulence in the ocean exhibits a degree of patchiness and is not statistically stationary in the vertical (Gregg, 1980).

Chapter Three attempts to relate the average shear spectral densities for $\mathrm{m}<\mathrm{m}_{\mathrm{c}}$ to the rate of dissipation of kinetic energy, $\varepsilon$, which is directly related to the integral of the shear spectrum for $\mathrm{m}>$ $\left(2 \pi \mathrm{L}_{0}\right)^{-1}$ by

$$
\varepsilon=7.5 v \int_{\left(2 \pi L_{o}\right)^{-1}}^{\infty} \frac{1}{2} S_{z} d m .
$$

In Section 4.3 we attempt to relate $\varepsilon$ to statistics of Richardson number $\left(\mathrm{Ri}=\mathrm{N}^{2} / \mathrm{S}^{2}\right)$ at a vertical scale roughly halfway between $\mathrm{m}_{\mathrm{c}}^{-1}$ and $\left(2 \pi \mathrm{L}_{0}\right)^{-1}$ such that $1.1 \leq<\mathrm{S}^{2}>/ \mathrm{N}^{2} \leq 1.2$. The vertical wavenumber bandwidth in which

$\left\langle\mathrm{S}^{2}\right\rangle=\int_{0}^{\mathrm{m}^{\prime}} \mathrm{S}_{\mathrm{z}} \mathrm{dm}=1.1-1.2 \mathrm{~N}^{2}$

is noted in Figure 4.1.

The $\mathrm{m}^{-1}$ region between $\mathrm{m}_{\mathrm{c}}$ and $\left(2 \pi \mathrm{L}_{\mathrm{o}}\right)^{-1}$ is, dynamically speaking, a no man's land. This part of the spectrum has been ascribed to a variety of processes including actively dissipating internal waves, propagation dominated by Doppler shifting and buoyancy modified turbulence. These latter theories consider the effects of stratification on universal, isotropic turbulence and differ primarily in the role of the potential energy flux (w'T') cospectrum (Holloway, 1983). Gregg et al. (1991) argue that the largest vertical scale influenced by buoyancy modified turbulence dynamics should be limited by the maxi- 
mum overturning scale, $2 \pi \mathrm{L}_{0}$, which is observed to be much smaller than the observed spectral cutoff, $\mathrm{m}_{\mathrm{c}}^{-1}$. A way of recasting this argument is to examine the scale separation between $\mathrm{m}_{\mathrm{c}}^{-1}$ and $2 \pi \mathrm{L}_{\mathrm{o}}$. Using the dissipation scaling determined in Chapter Three ( $\varepsilon=3.5 \times 10^{-10} \mathrm{~W} / \mathrm{kg} \mathrm{E}_{1}^{2} \mathrm{~N}^{2} / \mathrm{N}_{0}^{2}$, neglecting the frequency dependance), one finds $\mathrm{m}_{\mathrm{c}}^{-1} / 2 \pi \mathrm{L}_{\mathrm{o}}=32\left(\mathrm{~N} / \mathrm{N}_{\mathrm{o}}\right)^{1 / 2}$. Thus a large scale separation between the cutoff and turbulent scales is to be generally expected for the oceanic internal wave field.

A collection of ideas under the nominal title "saturated spectral theories" have been forwarded which suggest that the vertical wavenumber spectral density is limited by instability mechanisms (i.e. shear or advective instability) such that $\mathrm{mS}_{\mathrm{z}} / \mathrm{N}^{2}$ is a constant (Fritts, 1989). It is possible to provide an interpretation of this criterion using the eddy construct of Tennekes and Lumley (1987, p. 258259). If one considers a wave packet with wavelength $2 \pi / \mathrm{m}$ and envelope size $O \sim(1 / \mathrm{m})$, the spectral bandwidth of the wave packet will be $\mathrm{m}$ and a characteristic shear of the wave packet will be given by $\left(\mathrm{mS}_{\mathrm{z}}\right)^{1 / 2}$. Thus the criterion that $\mathrm{mS}_{\mathrm{z}} / \mathrm{N}^{2}$ be a constant can be interpreted as requiring the wave packet to retain a constant characteristic shear as it is fluxed to higher wavenumbers. The excess shear is presumably dissipated by the instability mechanisms.

The effects of Doppler shifting by larger scale waves on the small scale spectrum is discussed by Hines (1991d) using a statistical approach. That derivation, which did not include rotational effects, reveals an approximately $\mathrm{m}^{-1}$ range in the shear spectrum which results from the increasing ability of larger scale waves to Doppler shift the small scale wave field through zero frequency 
(i.e. $\mathrm{kU}-\omega=0$ ) as the wave scale is reduced. Small scale waves are removed from the spectrum by dissipation as they are Doppler shifted through zero frequency. The small scale waves are presumed to dissipate at vertical scales smaller than the inverse wavenumber corresponding to $<S^{2}>/ N^{2}=1$. In addition, the ray tracing model of Henyey, Wright and Flatte (1986), in which the interaction is entirely in the Doppler shift, appears to be in equilibrium with respect to an $\mathrm{m}^{-1}$ spectrum for $\mathrm{m}_{\mathrm{c}}<\mathrm{m}<2 \mathrm{~m}_{\mathrm{c}}$. Thus there are many competing dynamical explanations for the $\mathrm{m}^{-1}$ region.

Investigators generally assume the region of $m<\mathrm{m}_{\mathrm{c}}$ is dominated by internal wave/wave interactions (e.g. McComas and Muller (1981), Henyey et al. (1986) hereafter HWF, see also Chapter Three), and that energy transport from scales smaller than $\mathrm{m}_{\mathrm{c}}^{-1}$ to turbulent scales occurs via shear instability. We find evidence to support a transition between dynamical regimes which we interpret in favor of this scenario. In the ray tracing study of HWF, the energy flux in vertical wavenumber space is given by

$$
\mathrm{dm} / \mathrm{dt}=-\mathbf{U}_{\mathrm{z}} \cdot \mathbf{k}_{\mathrm{h}}
$$

where $\mathrm{m}$ and $\mathbf{k}_{\mathrm{h}}$ are the vertical and horizontal wavenumbers, respectively, of a test wave propagating in a background shear field, $\mathbf{U}_{\mathrm{z}}$. In that study the background was modeled as a GM internal wave field. See Chapter Three for further details. From the numerical simulations, HWF note that the presence of "critical layers" (regions in which the test wave wavenumber exceeds $2 \mathrm{~m}_{\mathrm{c}}$ in the model) is correlated with large shear events. Figure 4.2 is from Henyey (1984) demonstrating this issue. Henyey (1984) asserts that dissipations should be associated with these large shear events. A 

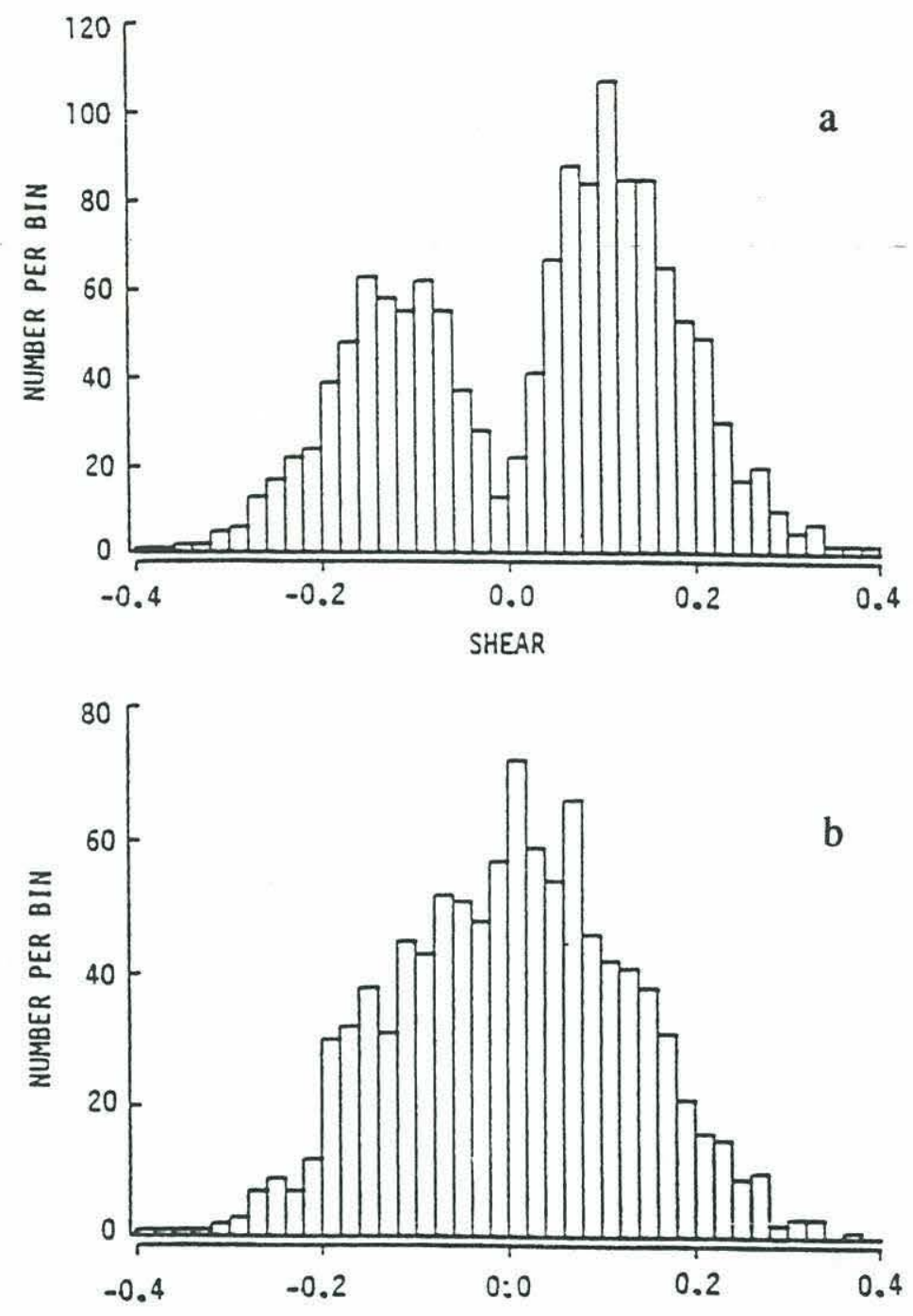

Figure 4.2: From Henyey (1984), a ray tracing study of small scale internal waves propagating in a background of larger scale internal waves. a) The probability distribution of background internal wave shear in regions in which the vertical wavenumber of the test wave exceeds $2 \mathrm{~m}_{\mathrm{c}}$, termed a critical layer event, and is declared to have 'broken'. b) The unconditional probability distribution of background shear. Henyey (1984) infers that large transports of energy to higher wavenumber and dissipation occur in regions of large background shear. 
second property of the model is that the $\mathrm{N}^{2}$ variability due to internal wave straining by the background waves was neglected in the simulations as the effects of this approximation were calculated to be energetically inconsequential (Henyey, 1983). This is reflected in the equation for the vertical wavenumber flux, equation 4.1. In as much as the strain variance is inconsequential to the model, the model implies that contours of dissipation in an $\mathrm{N}^{2}-\mathrm{S}^{2}$ plane should follow lines of constant shear squared in a region of constant ambient stratification.

Other models reveal similar characteristics. The induced diffusion and parametric subharmonic instability discussed by McComas and Muller (1981) have energy fluxes in vertical wavenumber space which are proportional to the vertical shear. As discussed by them, the parametric subharmonic instability mechanism dominates at low wavenumber and low frequencies and is characterized by the decay of a low wavenumber wave into two waves of half the frequency. This mechanism has a time scale of interaction which is proportional to the shear content of the double frequency wave. The induced diffusion mechanism dominates at high frequencies and large wavenumbers. It can be characterized as the scattering of a high frequency, high wavenumber wave by a low frequency, low wavenumber wave. This process acts as a diffusion of wave action in wavenumber space and is most efficient in vertical wavenumber. The diffusivity in vertical wavenumber space is proportional to the shear variance of the larger scale wave. Hence these models of internal wave-wave interactions also suggest that 
one would expect to find dissipations associated with larger $\mathrm{S}^{2}$ events, independent of $\mathrm{N}^{2}$.

By contrast, under the shear instability mechanism dissipations are expected to be associated with low $\mathrm{Ri}\left(\mathrm{N}^{2} / \mathrm{S}^{2}\right)$ events (e.g. Thorpe, 1973a). Due to the variability in $\mathrm{N}^{2}$ from internal wave straining these two criteria (large $\mathrm{S}^{2}$ and low $\mathrm{Ri}$ ) are not entirely similar. For a contour plot of dissipation versus $\mathrm{N}^{2}$ and $\mathrm{S}^{2}$ contours of dissipation are expected to follow lines of constant $\mathrm{Ri}$ under the shear instability scenario.

These expectations are born out by Figures 4.3 and 4.4. These figures are of bin averaged dissipations plotted versus $\mathrm{N}^{2}$ and $\mathrm{S}^{2}$ for forty WRINCLE profiles in the depth interval of $350-650 \mathrm{~m}$. The data are from the middle of the ring in a region of nominally constant background stratification: estimates of the ambient stratification $\left(\mathrm{N}^{2}\right)$ from pressure averaged temperature and salinity profiles average $.26 \times 10^{-4} \mathrm{~s}^{-2}$ with less than $20 \%$ variability. Hence the large variability of $\mathrm{N}^{2}$ on the vertical axis primarily represents the effects of internal wave straining on the stratification. In Figure 4.3, $\mathrm{N}^{2}$ and $\mathrm{S}^{2}$ were estimated with a $36 \mathrm{~m}$ least squares fit, which has a half-power point of approximately $60 \mathrm{~m}$ (see Figure A1.3, Appendix One), and in Figure 4.4 with a $6 \mathrm{~m}$ fit. The wavenumber cutoffs corresponding to the 36 and 6 meter fits are denoted in Figure 4.1. The $6 \mathrm{~m}$ fit is 24 times the Osmidov length when $\mathrm{L}_{0}$ is calculated as $L_{0}=\left(\left\langle\varepsilon>/ \bar{N}^{3}\right)^{1 / 2}\right.$. Consequently the 6 meter fit filters out the turbulent motions. If the outlying values on the plots are ignored, a transition from dissipations being a function of $S^{2}$ to a function of Richardson number as the scale is decreased from 


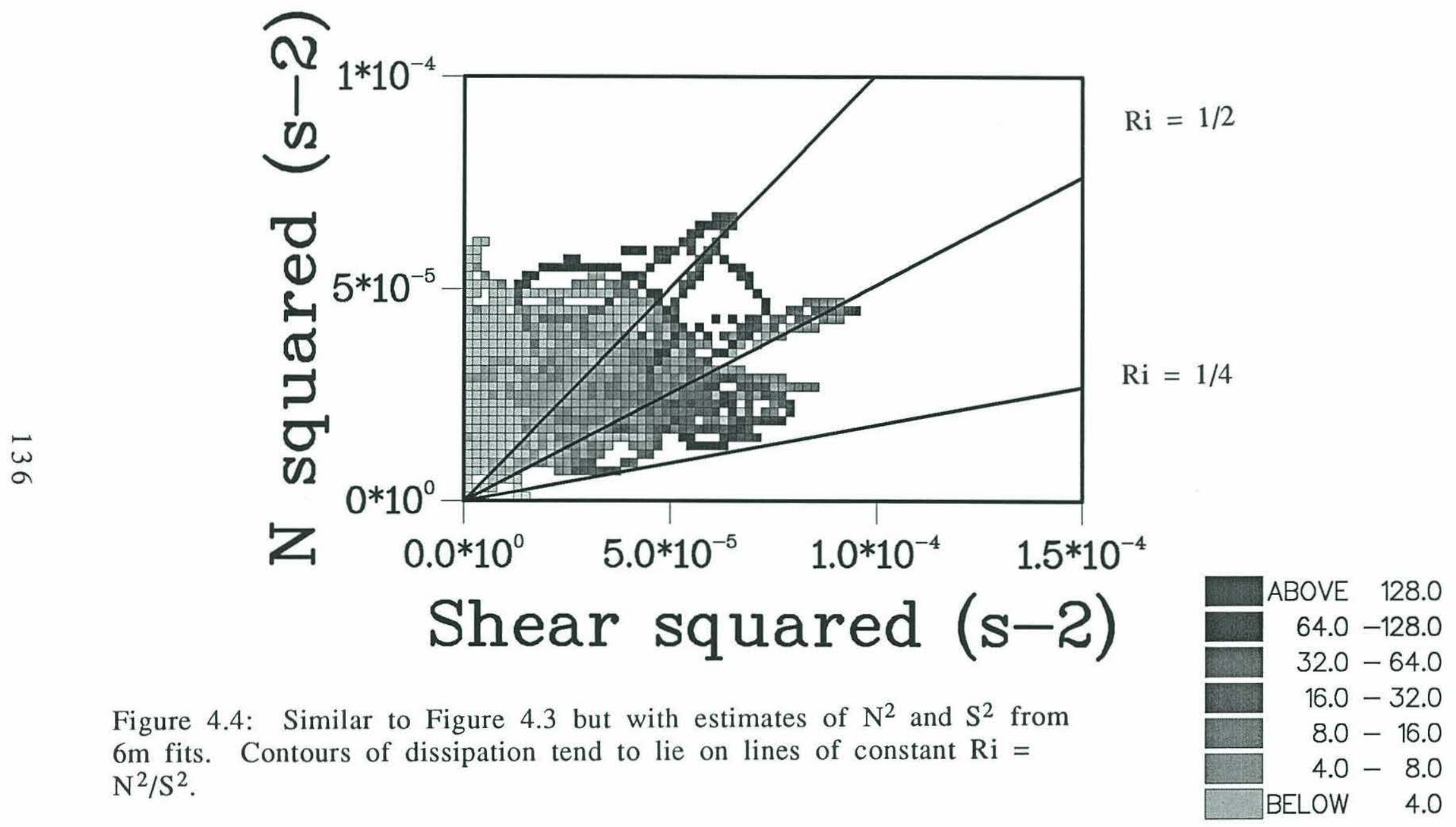




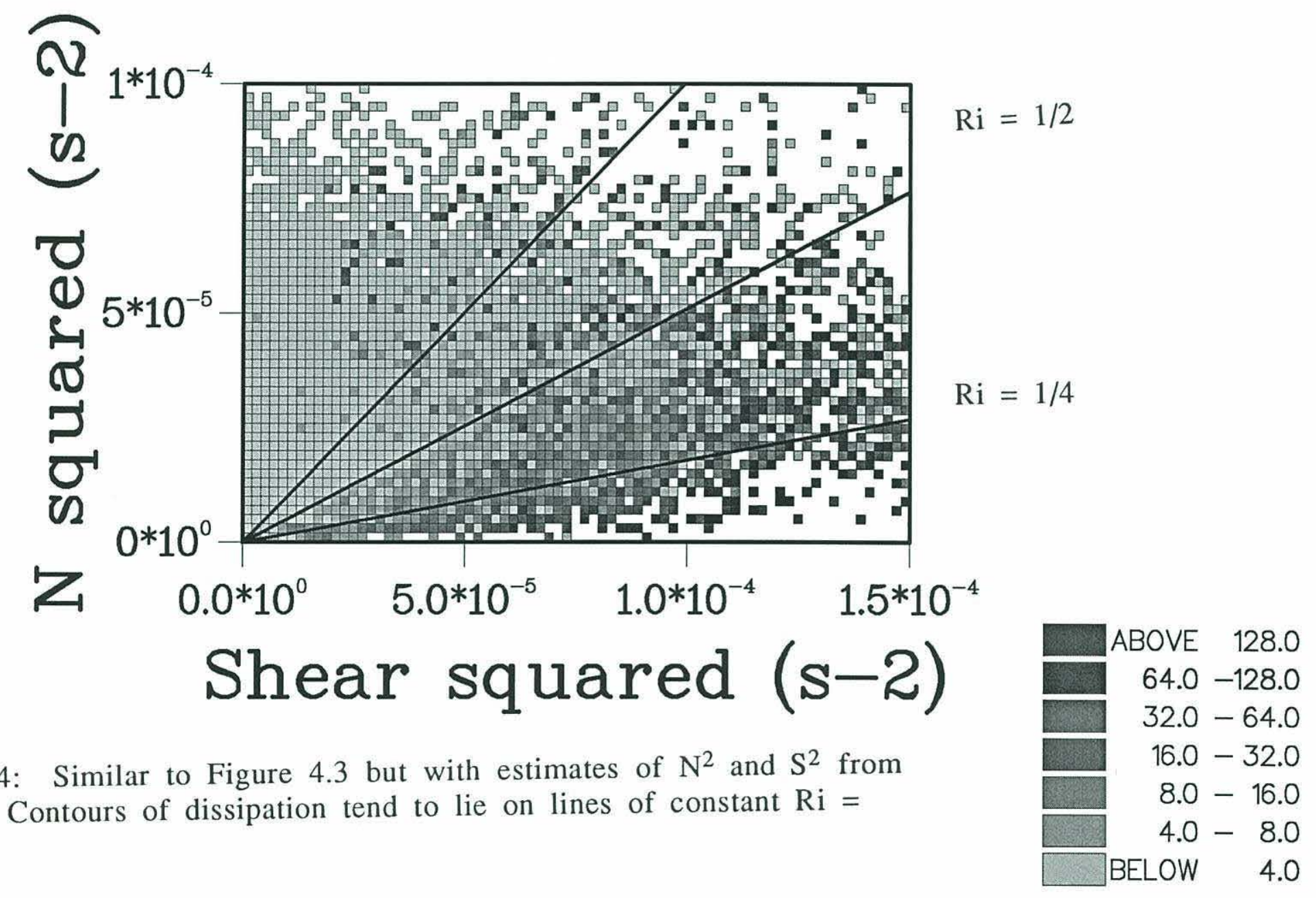

Figure 4.4: Similar to Figure 4.3 but with estimates of $\mathrm{N}^{2}$ and $\mathrm{S}^{2}$ from $6 \mathrm{~m}$ fits. Contours of dissipation tend to lie on lines of constant $\mathrm{Ri}=$ $\mathrm{N}^{2} / \mathrm{S}^{2}$. 
$\mathrm{m}_{\mathrm{c}}^{-1} / 1.3\left(36 \mathrm{~m}\right.$ fit, $\left.<\mathrm{S}^{2}>/ \mathrm{N}^{2}=.62\right)$ to $\mathrm{m}_{\mathrm{c}}^{-1} / 6.7\left(6 \mathrm{~m}\right.$ fit, $\left.<\mathrm{S}^{2}>/ \mathrm{N}^{2}=1.45\right)$ is apparent. We therefore conclude that there is a transition between dynamical regimes as the vertical scale is reduced and that shear instability plays a role in the upwavenumber transport of energy on vertical scales which are significantly larger than those exhibited by the turbulence. Since dissipation is associated with a turbulent momentum flux through a production/dissipation balance, the dissipations act to homogenize the velocity field and reduce the shear content of the wave field. The determination of the relative impact of the turbulent momentum fluxes on the background shear field as a function of the vertical scale (wavenumber) is addressed in Section 4.5.

\subsection{The Richardson Number Dissipation Parameterization of Kunze, Williams and Briscoe (1990)}

In Section 4.3 the Richardson number parameterization of the rate of dissipation of turbulent kinetic energy $(\varepsilon)$ developed by Kunze, Williams and Briscoe (1990), hereafter KWB, is examined. For a number of data sets exhibiting a broad range of energy levels, background stratifications and frequency content the KWB scaling is found to collapse the dissipation data to within a factor of four, the same as that claimed for the scaling of Henyey, Wright and Flatte (1986) in Chapter Three.

From a production/dissipation model of turbulent production via shear instability, KWB suggests: 
$\varepsilon=\mathrm{fr}(\Delta \mathrm{z})^{2}<\frac{\left(\mathrm{S}^{2}-4 \mathrm{~N}^{2}\right)}{24} \frac{(\mathrm{S}-2 \mathrm{~N})}{4}>=\frac{\mathrm{fr}(\Delta \mathrm{z})^{2}}{96}<\mathrm{N}^{3}\left(\frac{1}{\mathrm{Ri}}-\frac{1}{\mathrm{Ri}_{\mathrm{c}}}\right)\left(\frac{1}{\sqrt{\mathrm{Ri}}}-\frac{1}{\sqrt{\mathrm{Ri}_{\mathrm{c}}}}\right)>$

where $\mathrm{fr}$ is the fraction of the water column which is unstable, $\Delta \mathrm{z}$ is the interval over which $S^{2}$ and $\mathrm{N}^{2}$ are calculated, $\mathrm{S}^{2}$ is equal to $\left(\mathrm{U}_{\mathrm{z}}^{2}+\right.$ $\mathrm{V}_{\mathrm{z}}^{2}$ ), $\mathrm{S}$ is $\left(\mathrm{S}^{2}\right)^{1 / 2}$ and the brackets $<>$ represent an average over all supercritical events. The term $(\Delta z)^{2}\left(S^{2}-4 N^{2}\right) / 24$ represents the kinetic energy in a linear shear profile which would be dissipated if the turbulent fluxes increased the $\mathrm{Ri}$ profile back to another linear shear profile with $\mathrm{Ri}=1 / 4$. The term $(\mathrm{S}-2 \mathrm{~N}) / 4$ represents a time scale in which the excess kinetic energy is dissipated.

The phenomenological description of a shear instability event is usually posed as an initial value problem in a steady, uniform flow. The presence of a supercritical Richardson number (i.e. $\mathrm{Ri}<1 / 4$ ) permits the growth of small scale waves in that region. These waves grow until they reach a sufficient amplitude that they roll up, develop secondary instabilities, and then become turbulent (See Thorpe (1987) for a review.). The effect of the turbulence is to redistribute the momentum and buoyancy in the background to produce a background flow field with higher Richardson number.

The model of KWB represents an ad hoc scaling for the dissipation rate. In particular, it does not represent a model for the average dissipation in a shear instability event. We currently view the primary uncertainties to be the criterion $\mathrm{Ri}<1 / 4$ and the particular choice of time scales. The $\mathrm{Ri}<1 / 4$ criterion comes from the work of Miles (1961) and Howard (1961) as a general necessary but not sufficient criterion for linear instability. A nonlinear necessary and sufficient criterion for stability of $\mathrm{Ri}>1$ was deter- 
mined by Abarbanel et al. (1984). For small perturbations, laboratory data appear to be in fair agreement with the anticipated growth rates from linear theory ( e.g. Scotti and Corcos (1972), Thorpe (1971)) and support the $\mathrm{Ri}<1 / 4$ criterion. However, it is not clear what relevance this criterion has for velocity and density profiles in a time dependant environment in which not all of the shear and strain variance is resolved. Nor is it clear that the oceanic regime starts from a state of small perturbations. The criterion of Ri $<1 / 4$ for initial growth does not necessarily imply that, once turbulent, turbulence will not continue to extract energy from the mean flow if $\mathrm{Ri}$ increases beyond $1 / 4$. From laboratory studies of shear instability, Thorpe (1973b) observes the apparent collapse of turbulence when the background Richardson number exceeds .32 , independent of the initial Richardson number. Laboratory studies of grid generated turbulence in a uniform mean shear with stratification have found growing turbulence if the background $\mathrm{Ri}$ is less than $1 / 4$ and decaying turbulence if $\mathrm{Ri}$ exceeds $1 / 4$ (Rohr et al., 1988). Unfortunately this does not resolve the issue of the final state of the background Richardson number.

The second issue concerns the time scale on which the excess kinetic energy is dissipated. The time scale used by KWB comes from the work of Hazel (1972) for the linear instability of hyperbolic tangent velocity and density profiles. There is no particular reason why this linear instability time scale for the growth of small amplitude waves should be the time scale for the dissipation of the excess kinetic energy. The analysis of lab data by Thorpe (1973b) reveals that, after the inception of turbulence in a shear instability 
event, the inverse time scale of the resulting dissipation event was $.1 \mathrm{~N}^{2} / \mathrm{U}_{\mathrm{z}}$, independent of the initial Richardson number. This time scale is in general larger than the linear instability time scale.

Thorpe (1973a) proceeded to develop a model for the dissipation in a shear instability event from the above time scale and the difference in the energy content of the initial and final background velocity and density profiles. However, it is not clear at present if the studies of Thorpe involving shear layers are applicable to our oceanic measurements. as use of Thorpe's model requires the assessment of entrainment effects over the duration of a shear instability event. The dissipation model of KWB is more appropriately interpreted as a scaling relation rather than a model for the average dissipation rate associated with individual shear instability events.

The statistics of supercritical Richardson number events are quite sensitive to the vertical scale over which the Richardson number is calculated. Decreasing the vertical scale generally increases the estimate of rms shear and consequently decreases the Richardson number. This decrease is quite significant: for the WRINCLE data in Section 4.2, decreasing the vertical scale from $24 \mathrm{~m}$ to $6 \mathrm{~m}$, for example, increases the shear variance from $\left\langle\mathrm{S}^{2}\right\rangle=.84 \mathrm{~N}^{2}$ to $\left\langle\mathrm{S}^{2}\right\rangle=1.45 \mathrm{~N}^{2}$ and increases the observed percentage of $\mathrm{Ri}<1 / 4$ from 1.53 to $10.0 \%$.

We expect a Richardson number model for the rate of kinetic energy dissipation to be applicable over the scales where most of the shear in the internal wave band has been resolved. This appears to require resolving the shear over vertical scales in which $\left\langle\mathrm{S}^{2}>/ \mathrm{N}^{2}>1.0\right.$ (see below). On the other hand, one can, at least conceptually, choose 
too small of a vertical scale. One wishes to resolve those scales responsible for generating the turbulence, not those scales which reflect the turbulence itself. Hence we choose a scale for which $<\mathrm{S}^{2}>/ \mathrm{N}^{2}>1$, but not much more so.

The model of KWB is evaluated as a function of the vertical scale and Richardson number criterion for the WRINCLE data (Figure 4.5). The wavenumber cutoffs corresponding to the observed shear variances are denoted in Figure 4.1. The observed value of the dissipation rate is denoted by a straight line at $8.2 \times 10^{-9} \mathrm{~W} / \mathrm{kg}$. For a given Richardson number criterion the estimate of dissipation generally increases as $\Delta \mathrm{z}$ decreases, attains a maximum about a $\Delta \mathrm{z}$ giving $<\mathrm{S}^{2}>/ \mathrm{N}^{2} \sim 1$, and then finally begins to decrease at the smallest scales. Since $\left\langle S^{2}>/ N^{2}\right.$ increases as $\Delta z$ increases, increasing values of $\mathrm{S}^{2} / \mathrm{N}^{2}$ and fr offset the decreasing $\Delta \mathrm{z}$ in equation 4.2 , and the model estimate of dissipation is found to be rather insensitive to the vertical scale as long as $\left\langle S^{2}\right\rangle / N^{2}>1$. In order to produce consistent estimates of dissipation from a variety of data sets a variable $\Delta \mathrm{z}$ is used under the criterion that $1.1 \leq\left\langle\mathrm{S}^{2}>/ \mathrm{N}^{2} \leq 1.2\right.$.

The $\mathrm{Ri}_{\mathrm{c}}=1 / 4$ criterion underestimates the dissipation by a factor of four, Figure 4.5. As the Ri criterion increases, so does the model estimate for the dissipation. We refrain from making judgments as to the validity of one $\mathrm{Ri}$ criterion over the other.

The N-scaling dependance of KWB's parameterization (eq. 4.2) is examined in Figure 4.6. The data consist of the eight far field TOPO dives examined in Section 3.4 (Chapter Three) and are described more completely there. The relevant parameters are presented in Table 4.1. The observed dissipation rates were averaged for each 


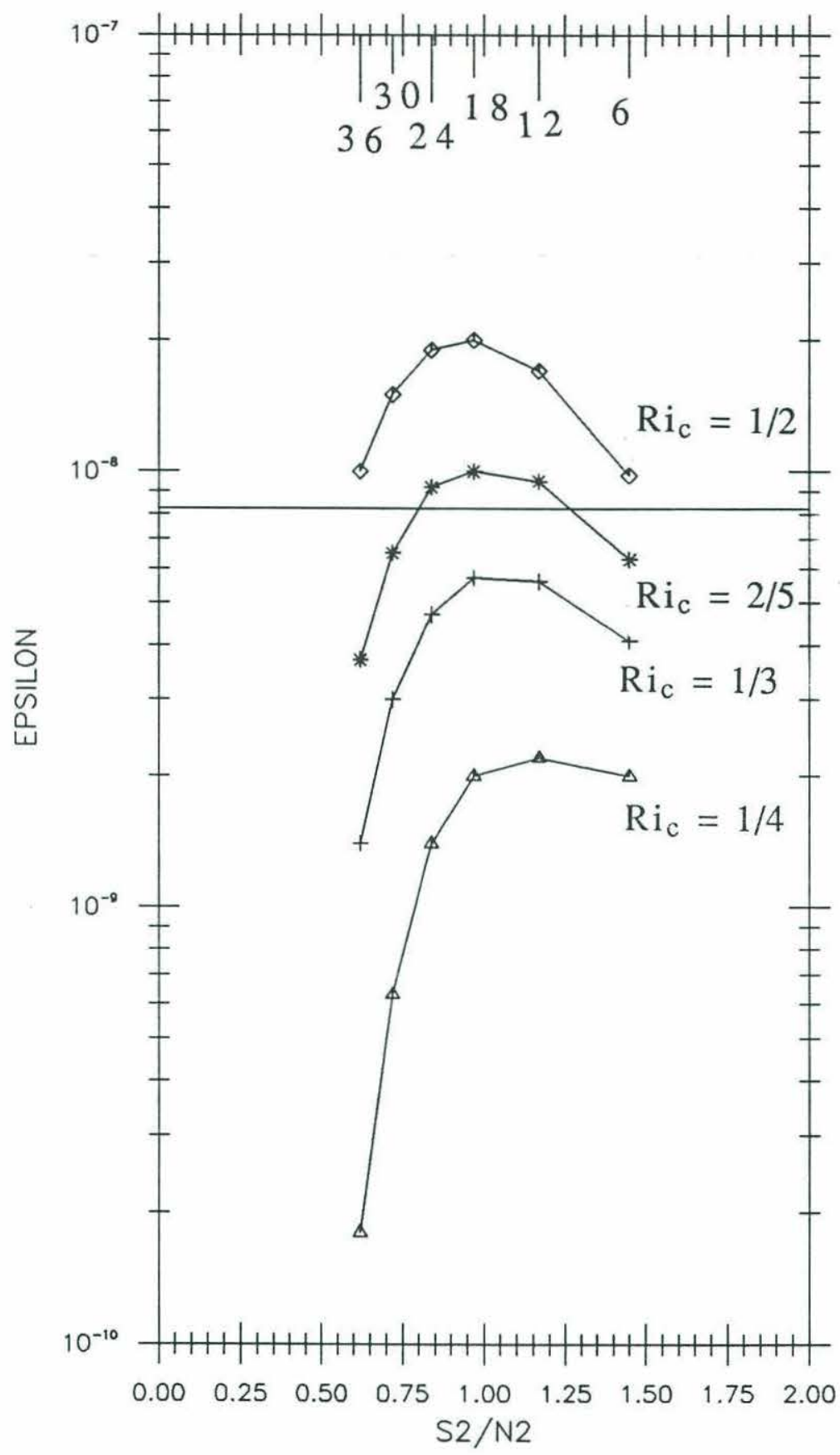

Figure 4.5: The dissipation parameterization of KWB evaluated as a function of $\Delta \mathrm{z}\left(<\mathrm{S}^{2}>/ \mathrm{N}^{2}\right)$ and $\mathrm{Ri}_{\mathrm{c}}$ for the WRINCLE data and plotted as a function of $\left\langle S^{2}>/ N^{2}\right.$. Estimates at a constant Richardson number are connected by lines as $\Delta \mathrm{z}$ is varied $\left(\mathrm{Ri}_{\mathrm{c}}=1 / 4,1 / 3,2 / 5\right.$ and $1 / 2$ are represented by $\Delta,+, *$ and $\Delta$ respectively, for fits of $\Delta z=36,30,24$, 18,12 and 6 meters). The observed value of the dissipation is denoted by a straight line at $8.2 \times 10^{-9} \mathrm{~W} / \mathrm{kg}$. At small $\Delta \mathrm{z}$ the $\mathrm{Ri}_{\mathrm{c}}=$ $1 / 4$ and $1 / 3$ estimates are scale independent. 


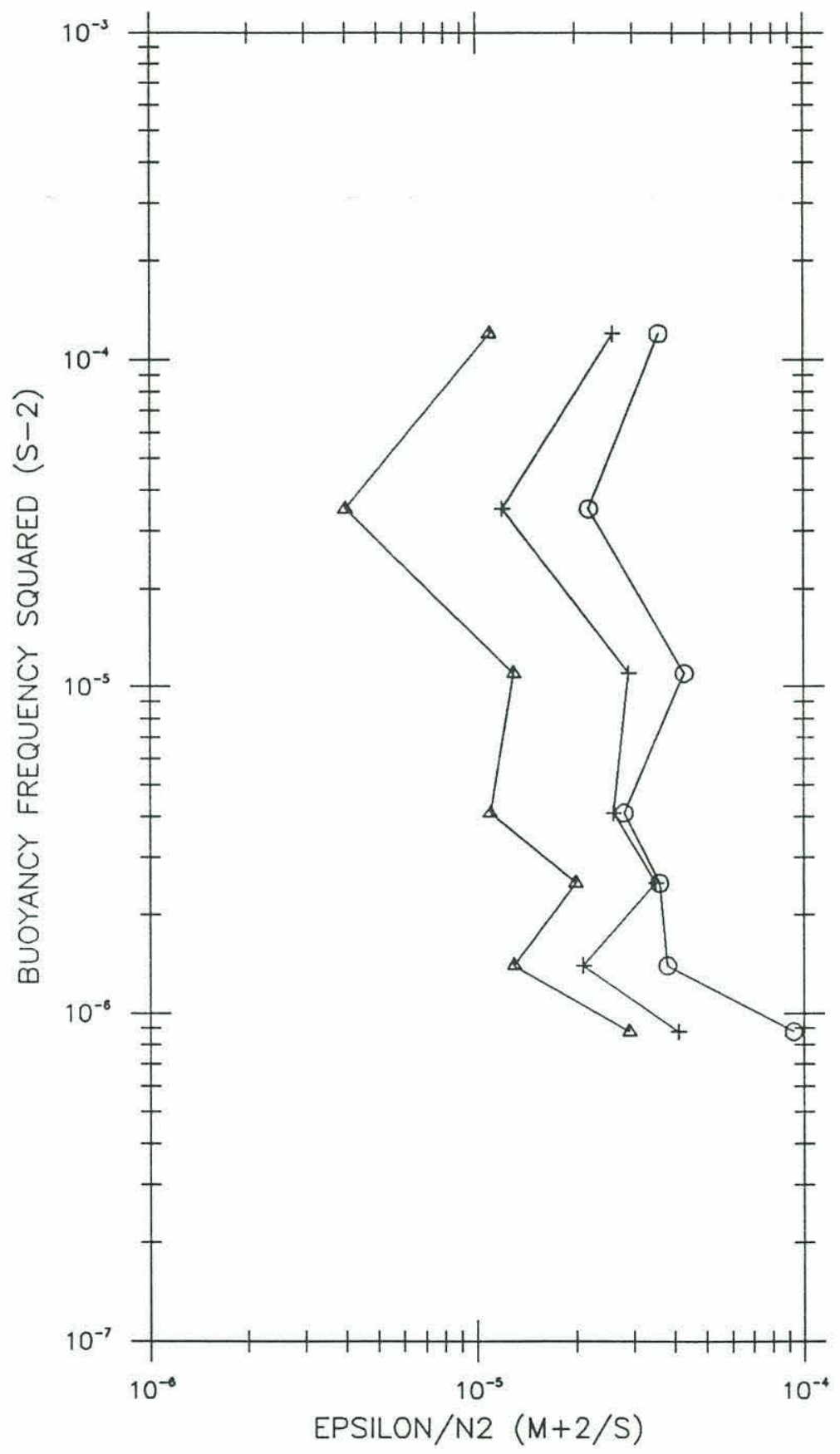

Figure 4.6: Observed dissipation values normalized by $1 / \mathrm{N}^{2}(0)$ and dissipation estimates from eq. 4.2 plotted against the buoyancy frequency squared using $\mathrm{Ri}_{\mathrm{c}}=1 / 4(\Delta)$ and $1 / 3(+)$ for the TOPO DEEP data set. The KWB scaling is found to collapse the observed dissipation estimates to within a factor of two. Compare with Figure 3.7. 
pressure range (D1-D7) and then divided by the observed average $\mathrm{N}^{2}$ for that range. Due to the variability in the background $\mathrm{N}^{2}$, the dissipation estimates for the TOPO_D2 and TOPO_D3 ranges were divided into 2 (3) segments of 100 (200) meters, respectively, the dissipation values averaged over that segment, normalized by the appropriate $\mathrm{N}^{2}$ and then averaged. Estimates of the dissipation from KWB's parameterization were obtained from least squares fits to the observed velocity and density profiles. A similar averaging scheme was then applied. The observed dissipation and the model estimates are displayed versus $\mathrm{N}^{2}$ in Figure 4.6. The dissipation parameterization generally underestimates the observed dissipation rate by a factor of three for the $\mathrm{Ri}<1 / 4$ criterion. Agreement is found to be good for the $\mathrm{Ri}<1 / 3$ criterion. The $\mathrm{Ri}_{\mathrm{c}}=1 / 3$ parameterization is seen to collapse the scatter in the data to within a factor of two. In Chapter Three the buoyancy scaling of the observed dissipation estimates was determined to be $\mathrm{N}^{2}$. Since there is a difference of a factor of 12 in the observed $\mathrm{N}$ values, we claim the KWB parameterization exhibits the same buoyancy scaling as the dissipation, $\mathrm{N}^{2}$.

The $(\mathrm{E},\langle\omega>)$ scaling of the KWB model is examined in Figure 4.7. Using the same data as in Figures 3.10 and 3.11 of Chapter Three (Table 4.1), the KWB scaling is found to collapse the data to within a factor of four, the same scatter as was observed for the HWF scaling of $\varepsilon \sim \mathrm{E}^{2} \mathrm{~N}^{2}\left(\omega^{2}-\mathrm{f}^{2}\right)^{1 / 2}$. 


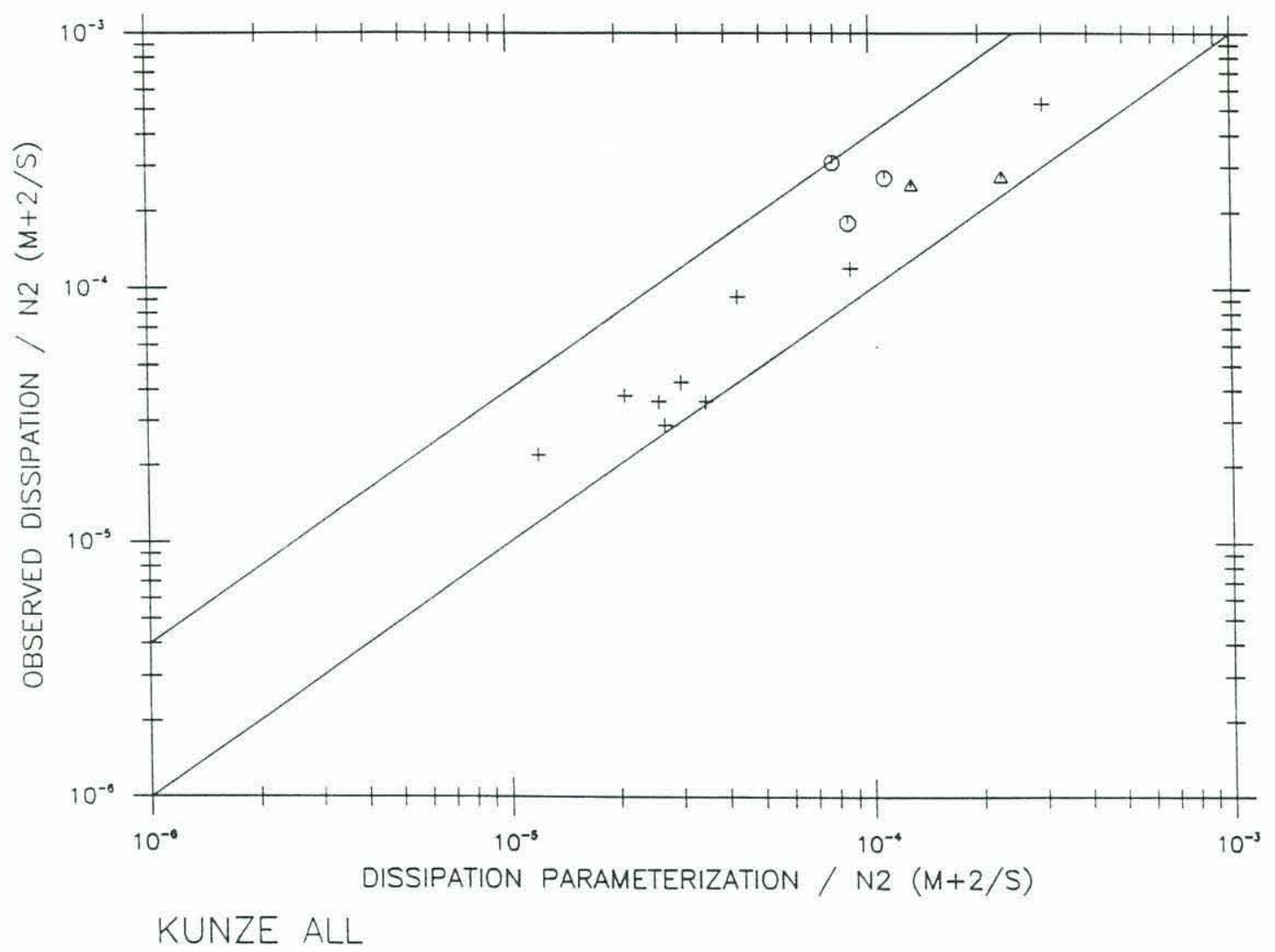

Figure 4.7: Dissipation estimates from KWB plotted against the observed dissipation rates for the data sets discussed in Chapter Two. The KWB parameterization is found to collapse the observed dissipation to within a factor of four, the same as that claimed for the HWF model. Compare with Figure 3.10. 
Table 4.1 Parameters for the Richardson number statistics

\begin{tabular}{lrlcc} 
& \multicolumn{1}{c}{ pressure } & $\left(\mathrm{N} / \mathrm{N}_{\mathrm{o}}\right)^{2}$ & $\Delta \mathrm{z}(\mathrm{m})$ & $<\mathrm{S}^{2}>/ \mathrm{N}^{2}$ \\
Topo_D1 & $100-200$ & 4.37 & 3 & 1.17 \\
Topo_D2 & $200-400$ & 1.22 & 3 & 1.16 \\
Topo_D3 & $400-1000$ & 0.38 & 4 & 1.18 \\
Topo_D4 & $1000-1500$ & 0.15 & 5 & 1.15 \\
Topo_D5 & $1500-2000$ & 0.092 & 5 & 1.21 \\
Topo_D6 & $2000-2500$ & 0.051 & 5 & 1.11 \\
Topo_D7 & $2500-3000$ & 0.031 & 6 & 1.15 \\
Topo_D6 & $2000-2500$ & 0.051 & 9 & 1.18 \\
Topo_D7 & $2500-3000$ & 0.031 & 18 & 1.16 \\
& & & & \\
Topo_F1 & $700-900$ & 0.32 & 11 & 1.15 \\
Topo_F2 & $900-1100$ & 0.22 & 7 & 1.12 \\
Topo_F3 & $1100-1300$ & 0.15 & 7 & 1.12 \\
& & & & \\
Wri_1+4 & $400-600$ & 0.97 & 8 & 1.19 \\
Wri_2+3 & $400-600$ & 0.95 & 15 & 1.13
\end{tabular}

\subsection{Statistics of the Richardson number}

In Section 4.3 the model of KWB was found to collapse a number of data sets exhibiting a wide range of energy levels (E), buoyancy frequencies $(\mathrm{N})$ and internal wave frequency content $(<\omega>)$. In Section 4.4 a number of progressively complicated expressions for the Richardson number probability density function (pdf) are examined with the intention of modeling the observed Richardson number distribution and obtaining an expression for the resulting $(\mathrm{E}, \mathrm{N},<\omega>)$ scaling of the KWB dissipation parameterization. 
Model 1: $\mathrm{S}^{2}$ as a $\chi_{2}^{2}$ variable and $\mathrm{N}^{2}$ constant.

Assuming that the two shear components are jointly normal, of equal variance and uncorrelated the following expression is obtained for the $S^{2}$ and Ri pdfs (e.g. Bendat and Piersol, 1986): Since

$\chi_{2}^{2}=\frac{\mathrm{U}_{\mathrm{z}}^{2}}{\left\langle\mathrm{U}_{\mathrm{z}}^{2}\right\rangle}+\frac{\mathrm{V}_{\mathrm{z}}^{2}}{\left\langle\mathrm{~V}_{\mathrm{z}}^{2}\right\rangle}=\frac{2 \mathrm{~S}^{2}}{\left\langle\mathrm{~S}^{2}\right\rangle}$ and $\mathrm{p}\left(\chi_{2}^{2}\right)=\frac{1}{2} \exp \left(-\chi_{2}^{2} / 2\right)$,

a standard change of variables gives

$\left.\mathrm{p}\left(\mathrm{S}^{2}\right)=<\mathrm{S}^{2}\right\rangle^{-1} \exp \left(-\mathrm{S}^{2} /<\mathrm{S}^{2}>\right)$

$\mathrm{p}\left(\mathrm{N}^{2} / \mathrm{S}^{2}\right)=\frac{\mathrm{N}^{2}}{\left\langle\mathrm{~S}^{2}\right\rangle}\left(\frac{\mathrm{S}^{2}}{\mathrm{~N}^{2}}\right)^{2} \exp \left(\frac{-\mathrm{N}^{2} \mathrm{~S}^{2}}{\left\langle\mathrm{~S}^{2}>\mathrm{N}^{2}\right.}\right)$.

An associated expression is the probability of occurrence of $\mathrm{Ri} \leq \mathrm{q}$, i.e. given $\left\langle S^{2}\right\rangle$, we want to find the percentage of points such that $S^{2}>$ $\mathrm{N}^{2} / \mathrm{q}$. The answer is simply:

$\%=\int_{\mathrm{N}^{2} / \mathrm{q}}^{\infty} \mathrm{p}\left(\mathrm{S}^{2}\right) \mathrm{dS}^{2}=\exp \left(-\mathrm{N}^{2} / \mathrm{q}<\mathrm{S}^{2}>\right)$.

The model only requires the estimation of the shear variance.

Model 2: Desaubies and Smith (1982)

In the course of their work, Desaubies and Smith provide an analytic expression for the Richardson number probability density $(\mathrm{p}(\mathrm{r}))$ and cumulative probability $(\mathrm{P}(\mathrm{r}))$ distributions. Assuming joint Gaussian, independent statistics for shear and strain the following expression were obtained:

$\mathrm{p}(\mathrm{r})=\frac{\mathrm{N}^{2} \lambda \exp \left[-1 / 2 \lambda^{2}\right]}{2 \sigma^{2} \mathrm{r}^{2} \sqrt{2 \pi}}\left\{1 \pm \mathrm{y} \sqrt{\pi}[1 \pm \varphi(\mathrm{y})] \exp \left(\mathrm{y}^{2}\right)\right\}$ 
$\mathrm{P}(\mathrm{Ri} \leq \mathrm{r})=\frac{1}{2}\left\{1-\varphi\left(\frac{1}{\sqrt{2} \lambda}\right) \pm[1 \pm \varphi(\mathrm{y})] \exp \left[\mathrm{y}^{2}\right] \exp \left[-1 / 2 \lambda^{2}\right]\right\}$

$y=(\sqrt{2} \lambda)^{-1}\left(1-\frac{N^{2} \lambda^{2}}{2 \sigma^{2} r}\right)$

$\varphi(y)=\operatorname{erf}(y)=\frac{2}{\sqrt{\pi}} \int_{0}^{y} \exp \left[-t^{-2}\right] d t$

where $r$ is the Richardson number, $U_{z}^{2}=V_{z}^{2}=\sigma^{2}$ and $\left\langle\eta_{z}^{2}\right\rangle=\lambda^{2}$. The \pm signs correspond to $r \gtrless 0$. The strain is related to the buoyancy frequency by $\eta_{\mathrm{z}}=\left(\left\langle\mathrm{N}^{2}>-\mathrm{N}^{2}\right) /<\mathrm{N}^{2}\right\rangle$ in a region of constant background $\mathrm{N}^{2}$. Hence, all that is required is an estimate of the shear and strain variances.

Model 3: $\mathrm{S}^{2}$ as a $\chi_{2}^{2}$ variable and $\mathrm{N}^{2}$ given by the Desaubies and Gregg (1981) expression.

Noting that displacements are found to have a Gaussian distribution (Briscoe, 1977), Desaubies and Gregg (1981) develop an expression for the distribution of temperature differences for a given differencing interval. The expression is nearly Gaussian for small strains. However, at large strains the distribution becomes markedly skewed towards low gradient events, as observed in the ocean. The expression for the equilibrium depth, y, of two isopycnals separated by a distance $\delta$ is:

$p(y \mid \delta)=\frac{1}{\sqrt{2 \pi}}\left\{\left(s^{\prime \prime}+\frac{\left[s+(\delta-y) s^{\prime}\right\}^{2}}{s^{3}}\right) \exp \left[\frac{-1}{2}\left(\frac{\delta-y}{s}\right)^{2}\right]\right\}$

where $\mathrm{s}(\mathrm{y})=\mathrm{Z}\{2[1-\rho(\mathrm{y})]\}^{1 / 2}$ and $\rho(\mathrm{y})$ is the lag correlation function which can be obtained from the cosine transform of the vertical 
wavenumber spectrum. Given a vertical wavenumber spectrum of the form $\left(\mathrm{m}^{2}+\mathrm{m}_{*}^{2}\right)^{-1}$, the lag correlation function is found to be $\exp \left(-\mathrm{m}_{*} \mathrm{y}\right)$. The variable $\mathrm{y}$ is related to the gradient by $\mathrm{g}=\overline{\mathrm{g}} \mathrm{y} / \delta$ where $\delta$ is the differencing interval and $\bar{g}$ is the (assumed) constant background gradient. The pdf of the gradient, $g$, is given by a simple change of variables: $\mathrm{p}^{\prime}(\mathrm{g} \mid \delta)=(\delta / \overline{\mathrm{g}}) \mathrm{p}(\delta \mathrm{g} / \overline{\mathrm{g}} \mid \delta)$. The function $\mathrm{s}(\mathrm{y})$ is equal to the rms value of the difference in displacement of two isotherms whose equilibrium separation is $\mathrm{y}$.

There are two parameters which need to be estimated: $\mathrm{m}_{*}$ and Z. Here we assume that $\mathrm{m}_{*}$ is given by the GM specification $\left(\mathrm{m}_{*}=3 \pi \mathrm{N} / \mathrm{bN}_{\mathrm{o}}=7.25 \times 10^{-3} \mathrm{~N} / \mathrm{N}_{\mathrm{o}}\right)$. We further note that a least squares fit of length $\delta$ to the density profile will result in a strain estimate of $\left\langle\eta_{\mathrm{z}}^{2}\right\rangle=2.26 \mathrm{~m}_{*} \mathrm{Z}^{2} / \delta$, assuming a spectra which is white until $\mathrm{m}=2 \pi / \delta$ (Appendix One). The rms displacement, $\mathrm{Z}$, is then estimated from $\mathrm{m}_{*}$ and the observed strain estimate. Following Toole and Hayes (1984), we have formed the Richardson number pdf from a Monte-Carlo simulation assuming the strain and two shear components are statistically independent.

It is now possible to examine the $(\mathrm{E}, \mathrm{N},\langle\omega\rangle)$ scaling for the KWB model for the Richardson number statistics. In general, if $\mathrm{S}^{2}$ and $\mathrm{N}^{2}$ are calculated over a scale $(\Delta \mathrm{z})$ which is some multiple of $\mathrm{m}_{\mathrm{c}}^{-1}$, then $<\mathrm{S}^{2}>/ \mathrm{N}^{2}$ and $\left\langle\eta_{\mathrm{z}}^{2}\right\rangle$ are independent of $\mathrm{N}$ and $\mathrm{E}$ for the canonical spectrum (Appendix Two). In model one (eq. 4.3) and two (eq. 4.4) it is obvious that if the variances $\left\langle S^{2}\right\rangle / N^{2}$ and $\left\langle\eta_{\mathrm{z}}^{2}\right\rangle$ are independent of $\mathrm{N}$, then so is the pdf of Ri. It is not so obvious that the Desaubies and Gregg expression for the strain pdf, equation 4.5, is independent of $\mathrm{N}$. In Figure 4.8 we have over plotted the model pdf for $\mathrm{N}=3$ and .3 


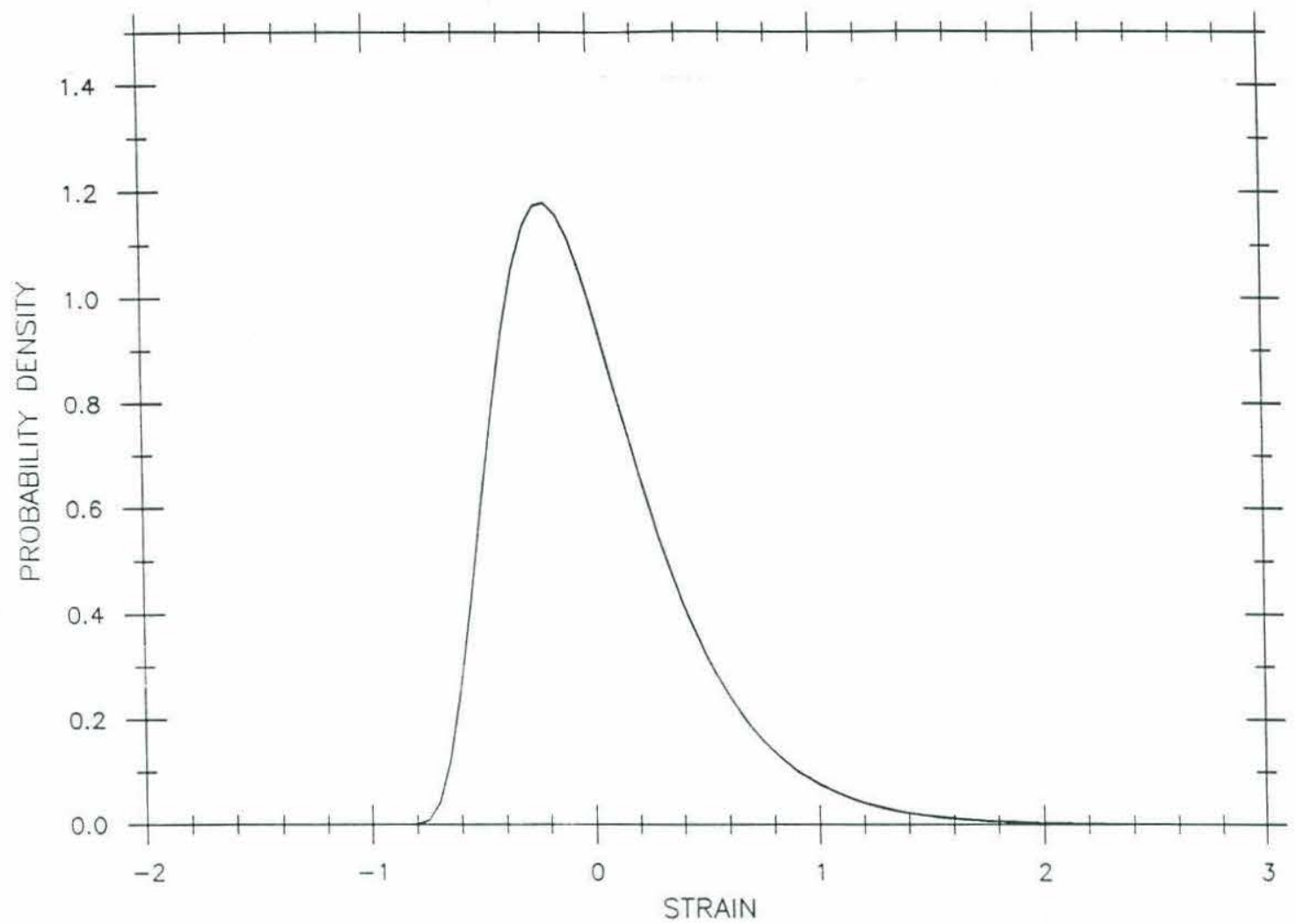

Figure 4.8: The Desaubies and Gregg strain pdf is estimated for two values of $\mathrm{N}$ ( 3 and $.3 \mathrm{cph}$ ) and the estimates are overplotted. It is assumed that both $\mathrm{Z}$ and $\mathrm{m}_{*}$ obey WKB scaling. There is little difference in the two pdfs. The model strain pdf is therefore independent of the stratification rate. 
cph where we have assumed that both $\mathrm{m}_{*}$ and $\mathrm{Z}^{2}$ obey WKB scaling. Values of $\delta=3 \mathrm{~m}$ and $\left\langle\eta_{\mathrm{z}}^{2}\right\rangle^{1 / 2}=.526$ were used. The two curves are indistinguishable. Thus, for at least small $\delta$, the Desaubies and Gregg strain pdf is independent of $N$. Since the $S^{2}$ pdf scales as $N^{2}$ it follows that the $\mathrm{Ri}$ pdf is also independent of $\mathrm{N}$ for model three.

Since the $\mathrm{Ri}$ pdf is independent of $\mathrm{N}$ it is obvious from equation 4.2 that the KWB dissipation parameterization scales as $\mathrm{N}^{3}$ for all three models. The requirement that $\Delta \mathrm{z}$ is a multiple of $\mathrm{m}_{\mathrm{c}}^{-1}$ gives the model scaling going as $\mathrm{E}^{2}$ since $\mathrm{Em}_{\mathrm{c}}$ is constant in the canonical shear spectrum. A frequency content scaling for these models would come from the ratio of shear to strain being a function of frequency, which would in turn be reflected in the Ri statistics. Since the particular form of the frequency dependence is not important for the arguments which follow, we simply write $\varepsilon \sim E^{2} N^{3} g(\omega)$.

This scaling is distinct from the $\mathrm{E}^{2} \mathrm{~N}^{2}\left(\omega^{2}-\mathrm{f}^{2}\right)^{1 / 2}$ scaling of the HWF model, and yet we find that when applied locally to observed velocity and density profiles both collapse data exhibiting over an order of magnitude variability in N. (Compare Figures 4.6 and 3.7.) The scalings developed for the KWB parameterization from the three statistical models are inconsistent with the observations. We therefore conclude that a buoyancy scaling which is not addressed by the three Richardson number probability distributions must be implicit in the observed $\mathrm{Ri}$ statistics. We further note that this change need not be obvious: the expression of KWB is cubic in $\mathrm{S}$ and fr is exponentially dependant on $\left\langle\mathrm{S}^{2}>/ \mathrm{N}^{2}\right.$. We are led to examine the $\mathrm{Ri}$ statistics to determine the source of the departure from the nominal scalings. 
To examine the model assumptions, a data set in which the data are statistically homogeneous and which contains as many degrees of freedom as possible is desired. Ideally an examination of the Richardson number model assumptions would include the issue of buoyancy scaling of the $\mathrm{S}^{2}$ and $\mathrm{N}^{2}$ pdfs as well as that of the statistical independence between the strain and shear components. Unfortunately there is insufficient data in the TOPO_DEEP data set to resolve the issue of the potential buoyancy scaling of the shear and strain pdfs and we are limited at this point to examining the assumption of statistical independence at a single value of the buoyancy frequency. A data set of 29 profiles taken from the main thermocline (550-900 db) of the North Atlantic subtropical convergence zone during Feb-March of 1986 (FASINEX) is chosen with which to address this issue. The vertical variability in the buoyancy frequency squared formed from pressure averaged temperature and salinity profiles is a factor of $5 \%$, much less than the variability due to internal wave straining. These data have the additional qualification of being the more GM-like than either the WRINCLE or TOPO data sets. Thus the conclusions of this study should be applicable to most of the mid-latitude oceans. For $3 \mathrm{~m}$ least square fits the following values are obtained:

$<\mathrm{N}^{2}>=2.03 \times 10^{-5} \mathrm{~s}^{-2}$

$\left\langle\eta_{\mathrm{z}}^{2}\right\rangle=\left\langle\left(\left(<\mathrm{N}^{2}>-\mathrm{N}^{2}\right) /<\mathrm{N}^{2}>\right)^{2}>1 / 2=.526\right.$

$<\mathrm{S}^{2}>=1.61 \times 10^{-5} \mathrm{~s}^{-2}$

The three $\mathrm{Ri}$ models disagree markedly with respect to their predictions of the probability of occurrence of $\mathrm{Ri} \leq 1 / 4$ for the FASINEX parameters discussed above, Figure 4.9. Most notably, the 


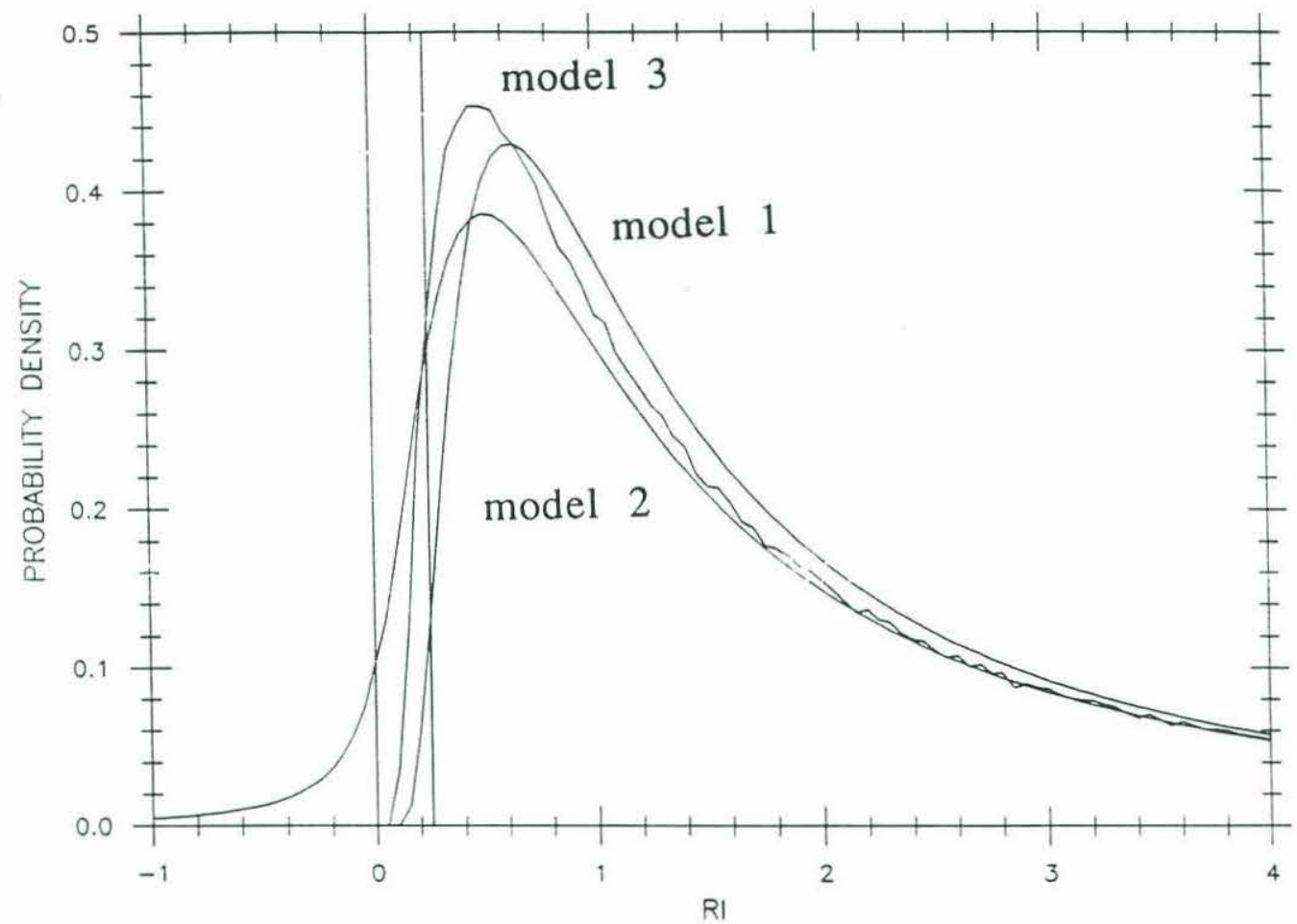

Figure 4.9: The three Richardson number model pdfs. Model one has the smallest percentage of $\mathrm{Ri} \leq 1 / 4$, and the Desaubies and Smith model (model two) the largest. The Desaubies and Smith model is also notable for the frequent occurrence of unstable stratification associated with the Gaussian strain pdf. 
lack of $\mathrm{N}^{2}$ variability in the first model results in a small percentage of $\mathrm{Ri}<1 / 4(.69 \%)$ and the Desaubies and Smith model (\#2) $(7.79 \% \mathrm{Ri} \leq$ 1/4) has a large fraction of events with negative buoyancy frequency associated with the Gaussian strain pdf. The Desaubies and Gregg$\mathrm{N}^{2}, \chi_{2}^{2}-\mathrm{S}^{2}$ model (\#3) is noted to have $3.60 \% \mathrm{Ri} \leq 1 / 4$. From this we conclude that an accurate assessment of the $\mathrm{Ri}$ pdf requires the inclusion of internal wave strain and that this variability needs to be appropriately modeled.

Two Richardson number pdfs have been estimated from the data. The first was formed by calculating the local values of $\mathrm{S}^{2}, \mathrm{~N}^{2}$ and $\mathrm{Ri}\left(\mathrm{N}^{2} / \mathrm{S}^{2}\right)$ from $3 \mathrm{~m}$ least squares fits to velocity and density. The samples were subsequently binned versus Ri. This pdf is termed 'local'. The second pdf was formed by randomly sampling the $S^{2}$ and $\mathrm{N}^{2}$ distributions, forming $\mathrm{Ri}$ and then binning the resulting Richardson numbers. The results are presented in Figure 4.10. The two distributions differ markedly for $\mathrm{Ri}<1 / 4$. The percentage of $\mathrm{Ri}<$ $1 / 4$ for the local pdf is $1.9 \%$ in comparison to an estimate of $5.1 \%$ for the randomly sampled population. This difference is statistically significant at the $95 \%$ confidence interval using a Pearson-Clopper estimator (Bradely, 1968). The randomly sampled pdf is in fair agreement with the Desaubies and Gregg- $\mathrm{N}^{2}, \chi_{2}^{2}-\mathrm{S}^{2}$ model (Figure 4.11). In the region $\mathrm{Ri}<.4$ the local pdf appears to agree best with the constant-N, $\chi_{2}^{2}-S^{2}$ pdf (Figure 4.12).

The joint pdf for two Gaussian variables is completely specified by the means, variances and covariances. Thus two Gaussian variables are statistically independent if their covariance is zero. The FASINEX shear values are well described by $\chi_{2}^{2}$ statistics. Both 


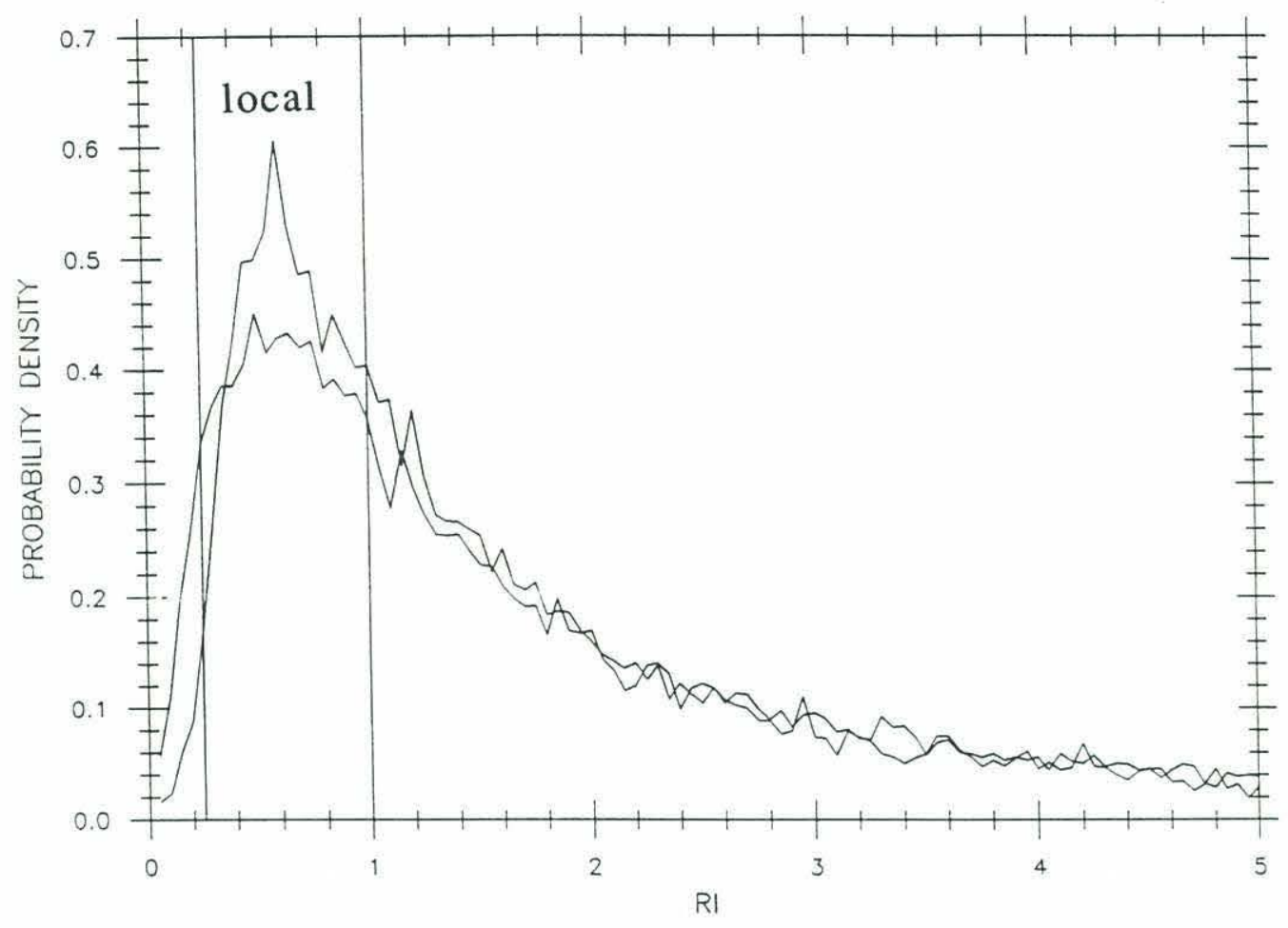

Figure 4.10: The local and randomly sampled Richardson number pdfs for the 29 FASINEX profiles in the depth interval $550-900 \mathrm{~m}$. The two differ markedly for $\mathrm{Ri} \leq 1 / 4$ : the local pdf exhibits $1.9 \% \mathrm{Ri} \leq$ $1 / 4$ and the randomly sampled $5.1 \%$. 


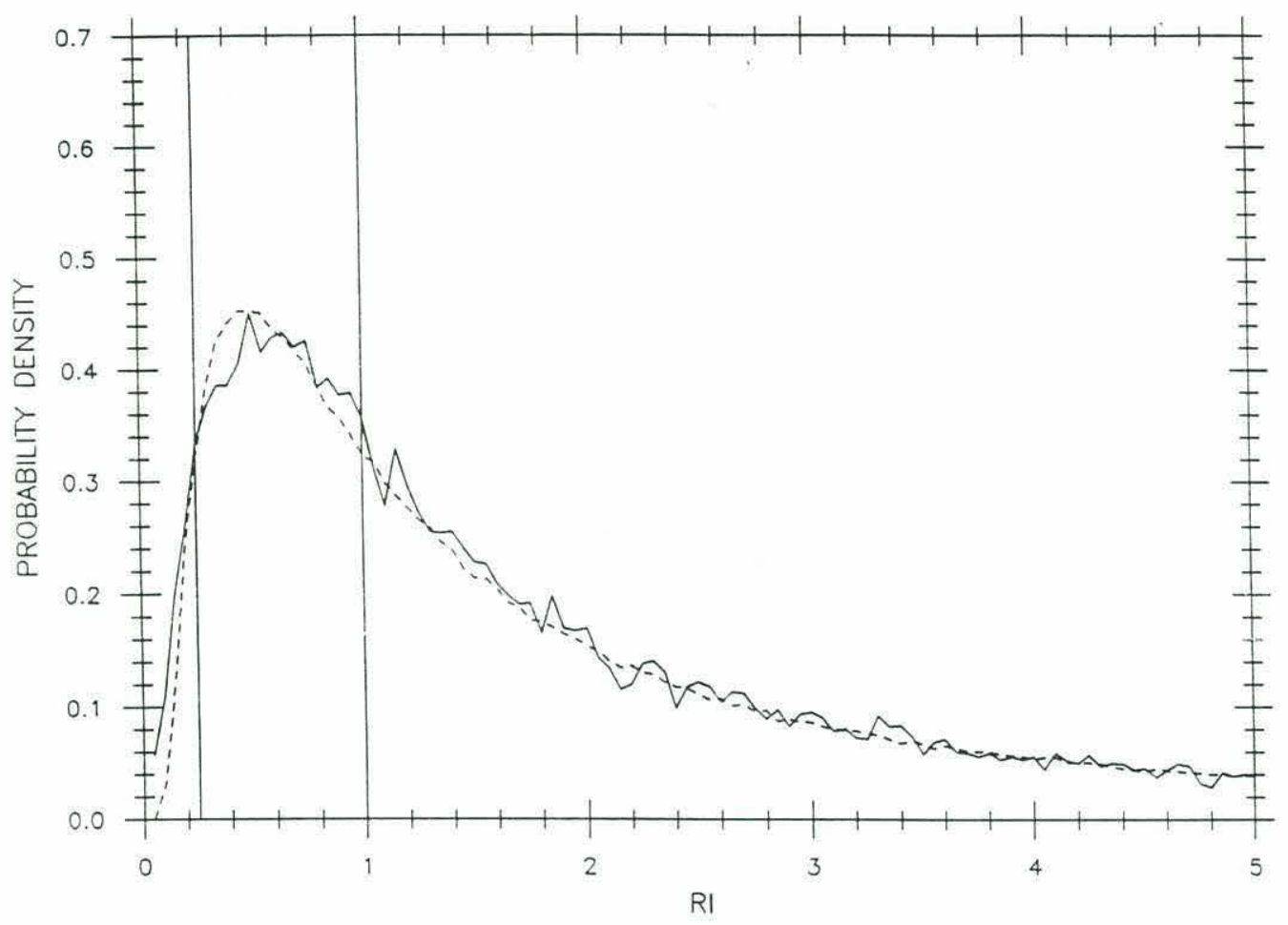

Figure 4.11: The randomly sampled Richardson number pdf for the FASINEX data (solid line) and the Desaubies and Gregg- $\mathrm{N}^{2}, \chi_{2}^{2}-\mathrm{S}^{2}$ Richardson number model. 


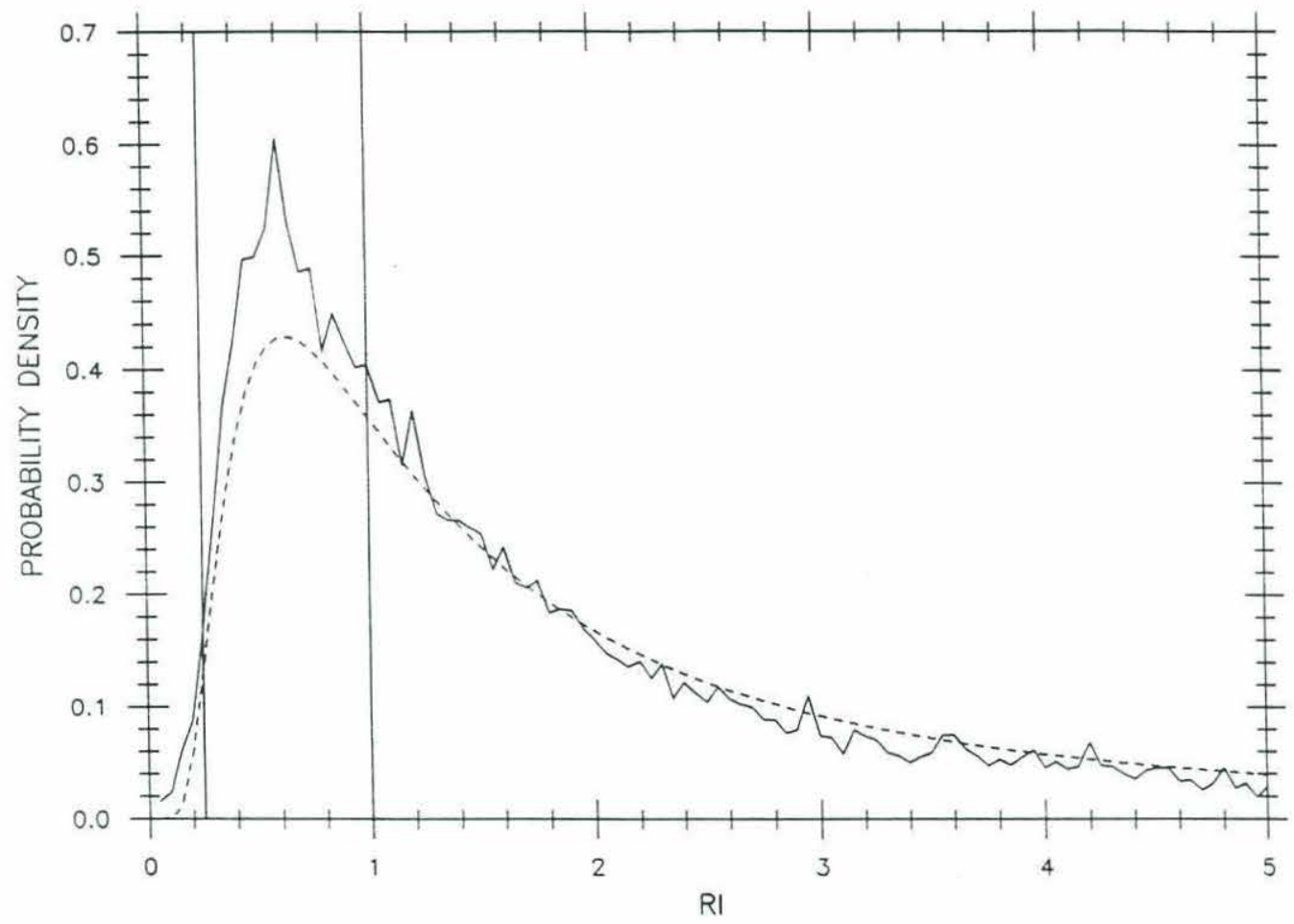

Figure 4.12: The local Richardson number pdf for the FASINEX data (solid line) and the constant- $\mathrm{N}, \chi_{2}^{2}-\mathrm{S}^{2}$ Richardson number model (model one). 
shear components have zero means, identical variances, are Gaussian and uncorrelated, leading to a $\chi_{2}^{2}$ distribution (Figure 4.13). As noted by many other researchers (e.g. Sherman and Pinkel, 1991; Desaubies and Gregg, 1981) the observed strain pdf is non-Gaussian (Figure 4.14). For non-Gaussian variables, the absence of a correlation does not imply statistical independence (e.g. Bendat and Piersol, 1986). The shear/strain covariance is not statistically different from zero for these data. Most other investigators have obtained similar results (Evans (1982), Toole and Hayes (1984)). However, since the strain is non-Gaussian, it can not concluded that the shear and strain are statistically independent. From the difference between the local and randomly sampled pdfs, (Figure 4.10), $\mathrm{N}^{2}$ and $\mathrm{S}^{2}$ are found to be statistically dependant.

Such a dependance has traditionally been examined in the light of adiabatic processes. Possible explanations include inhomogeneity and anisotropy in the internal wave field (Willebrand et al., 1977), phase locking from non-linear interactions (Desaubies and Smith, 1982), and the straining of a low frequency shear by a high frequency strain (Kunze et al., 1990). Here, however, a diffusive explanation is pursued and it is determined that the noted statistical dependance represents the effects of a turbulent momentum flux which reduces the shear and drives the Richardson number to larger values.

In order to address where in $S^{2}-\mathrm{N}^{2}$ space the dependence occurs 2-D pdfs have been prepared for the local and randomly sampled data sets. In Figure 4.15 (a), the local pdf is shown in counts. In Figure 4.15 (b), the difference (in counts) between the independent 


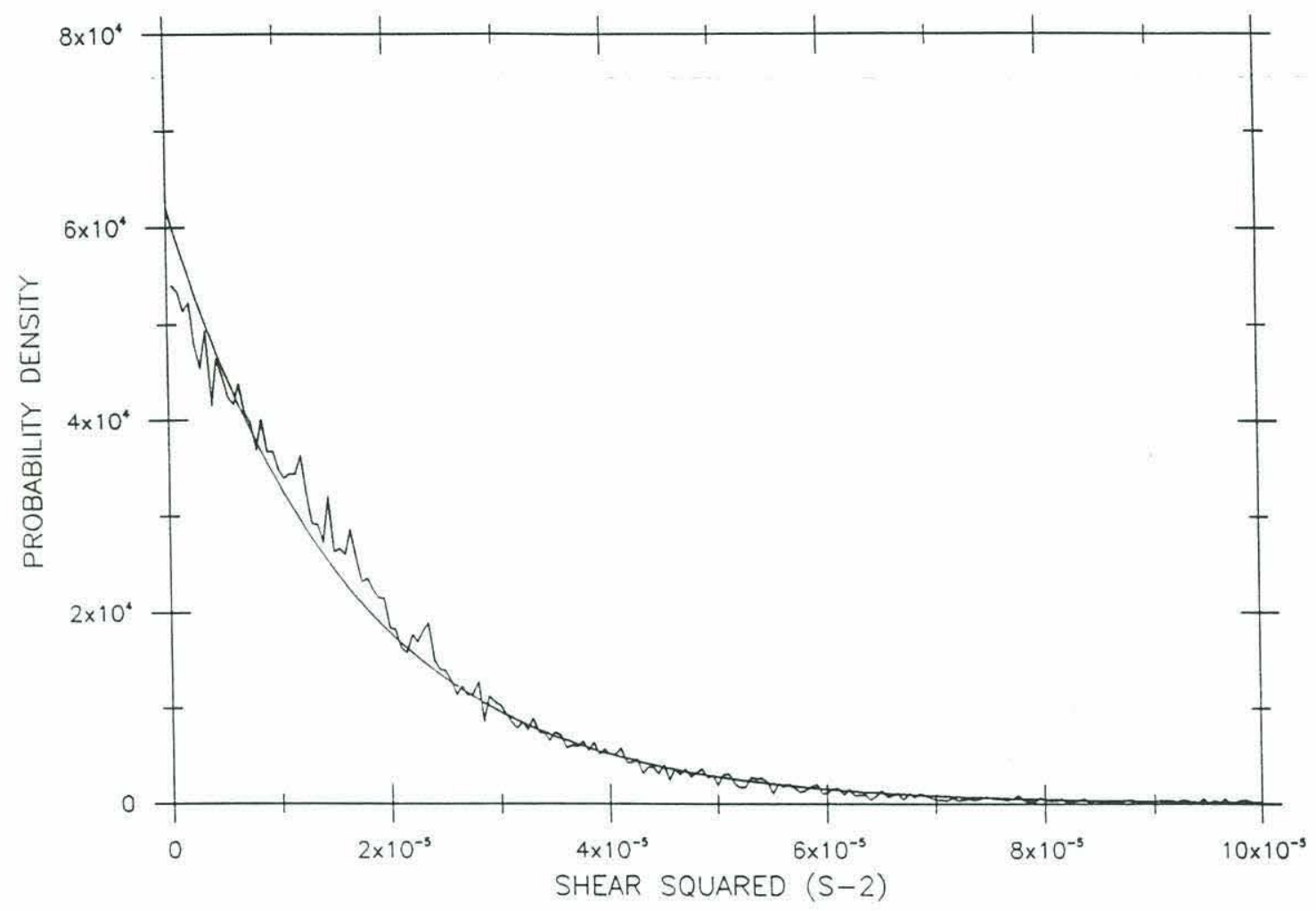

Figure 4.13: The FASINEX $S^{2}$ pdf and the $\chi_{2}^{2}-S^{2}$ model from eq 4.3. The two agree sufficiently well to justify the use of Gaussian, uncorrelated statistics for the shear. 


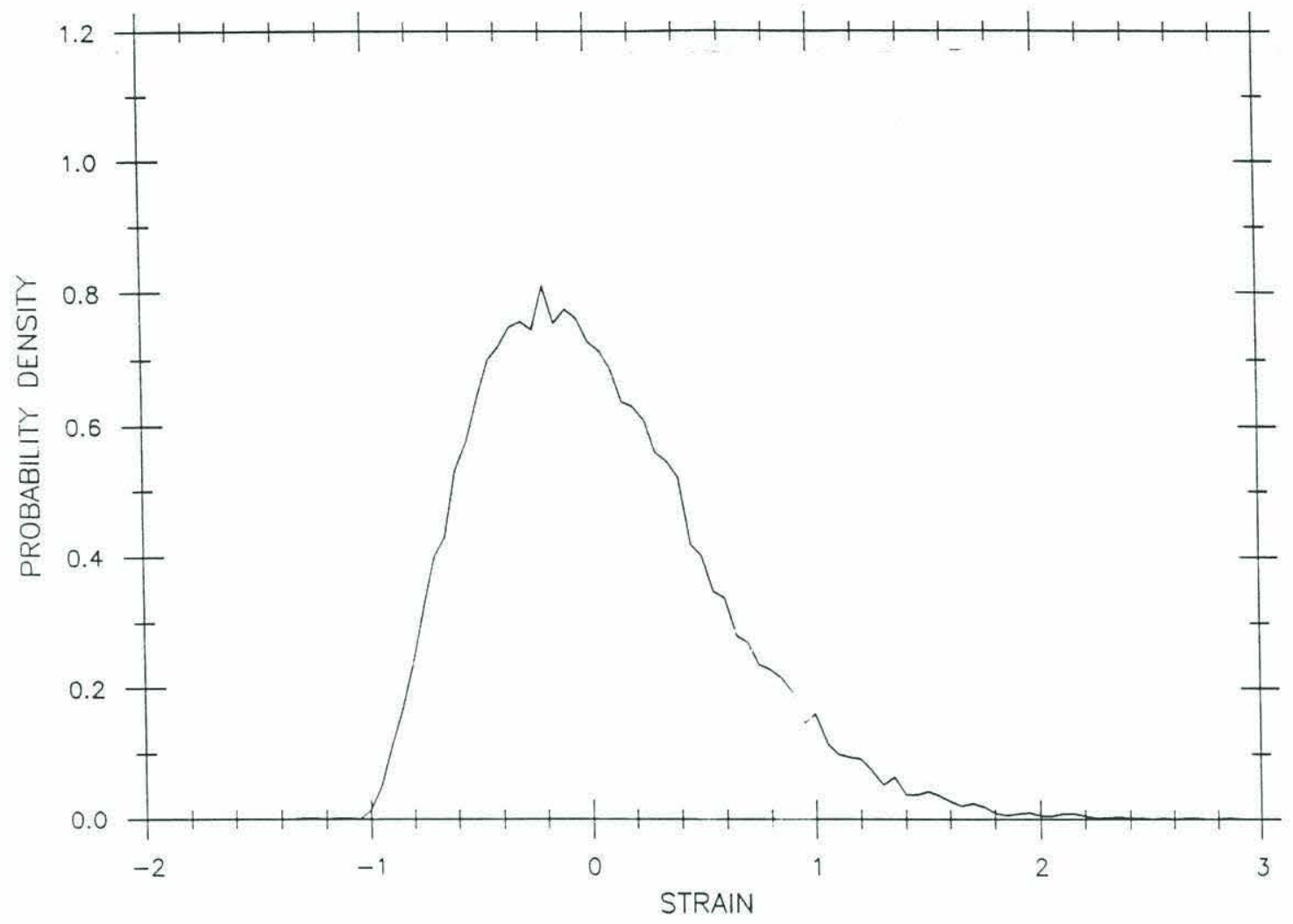

Figure 4.14: The observed FASINEX strain pdf (solid line). The observed pdf is notably skewed toward low gradient events. 


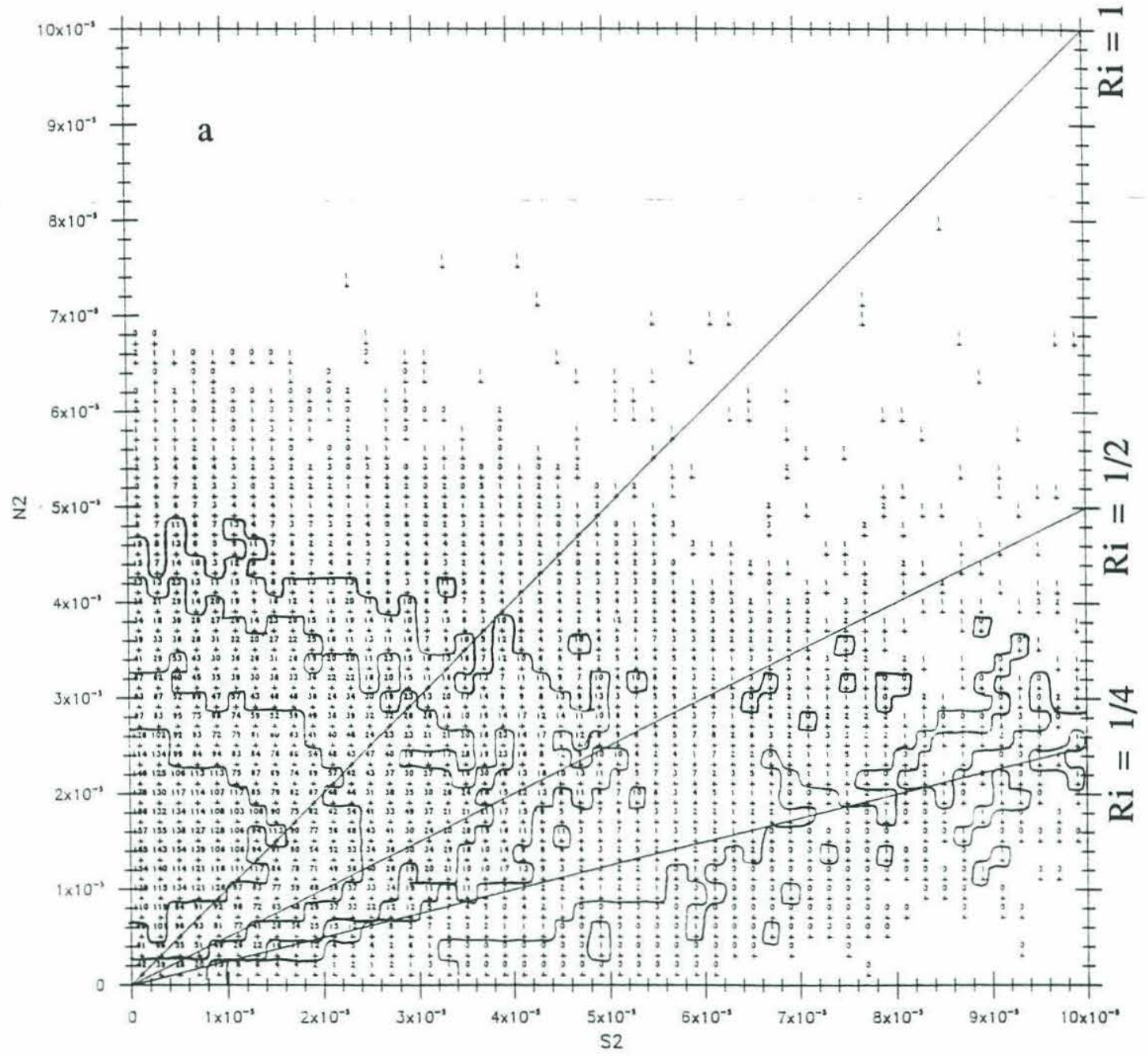

Figure 4.15: a) The 2-D N $\mathrm{N}^{2}-\mathrm{S}^{2}$ pdf for the FASINEX data in the depth range $550-900 \mathrm{~m}$. The straight lines emanating from the origin are lines of constant Richardson number. Contour levels are 0, 10, 20, 50 and 100 counts. b) The difference (in counts) between the randomly sampled and local pdfs. The statistical dependence between $\mathrm{N}^{2}$ and $\mathrm{S}^{2}$ results in a loss of $\mathrm{Ri} \leq 1 / 3$ and higher than average $\mathrm{N}^{2}, \mathrm{~S}^{2}$ events. The loss of $\mathrm{Ri} \leq 1 / 3$ is interpreted as a dissipative effect. 


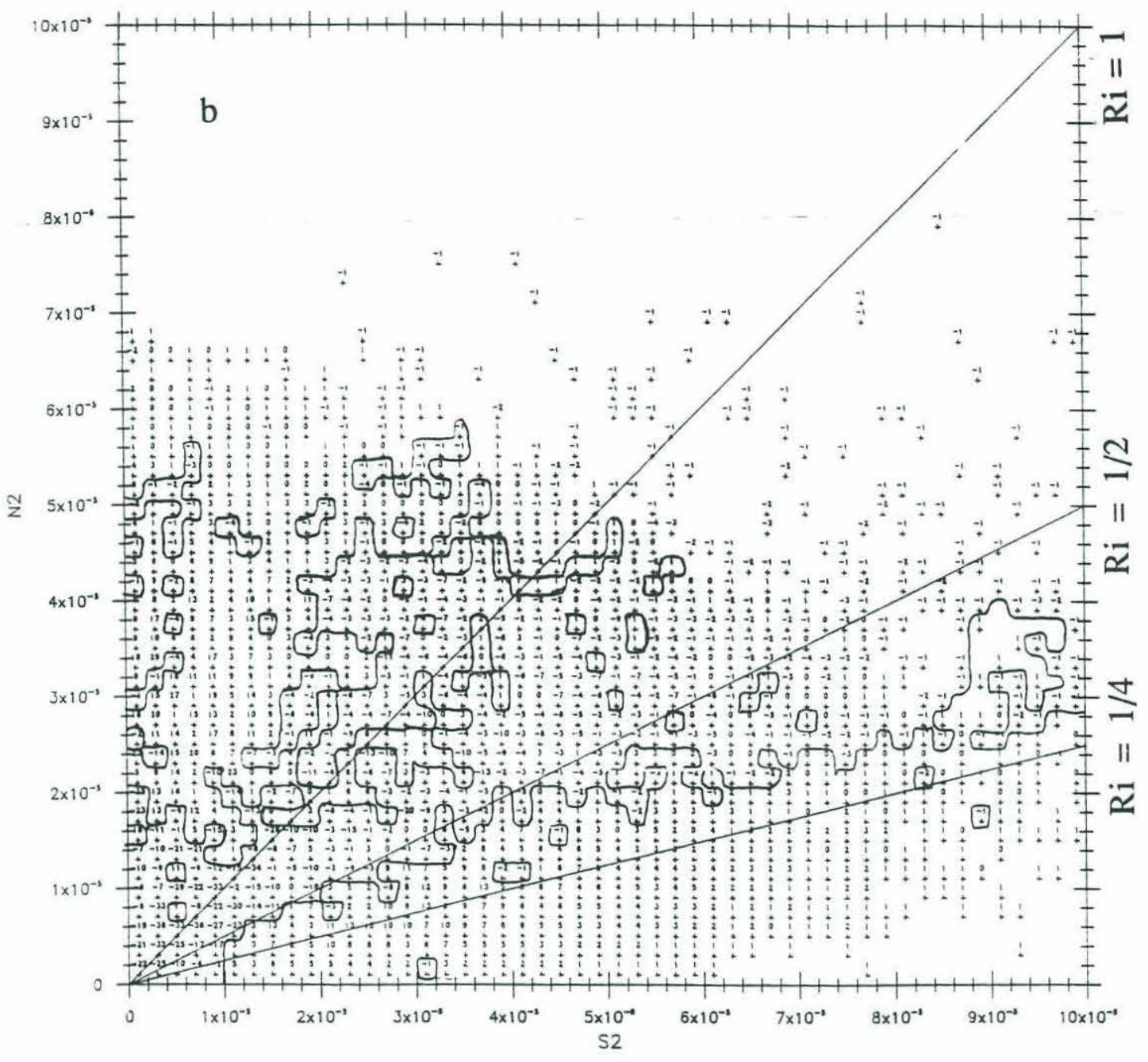


version and the local version is shown. In Figure 4.15 the signature of the dependance is found to be a reduction in the local population of $\mathrm{Ri}<1 / 3$ events. This reduction appears to be consistent over all $\mathrm{N}^{2}$ values. The difference plot $(4.15$ (b)) shows the randomly sampled version to have a more uniform distribution. Also indicated is a loss of low $\mathrm{S}^{2}$ for higher than average $\mathrm{N}^{2}$ events. One possible explanation for this low $\mathrm{S}^{2}$, high $\mathrm{N}^{2}$ deficit is the strain of a high frequency wave acting upon a near-inertial shear in such a manner as to decrease the Richardson number for high N (KWB's equation 5). The fractional loss of low $\mathrm{Ri}$ events is much larger than that of low $\mathrm{S}^{2}$, higher than average $\mathrm{N}^{2}$ events.

The loss of low Ri events is interpreted as being due to diffusive effects. As Ri becomes supercritical in a time dependant field, the linear shear instability arguments predict the growth of unstable waves. If the region of instability persists for a long enough time the unstable waves will eventually break and dissipate. For the $6 \mathrm{~m}$ WRINCLE data in Figure 4.4 dissipations are associated with low Richardson number values. From production/dissipation balances, momentum and buoyancy fluxes are associated with the dissipation. We propose that these momentum and buoyancy fluxes in turn alter the Richardson number distribution to that which is observed by reducing the shear and buoyancy gradients. Under the assumption of small flux Richardson number the momentum fluxes are much larger than the buoyancy fluxes and the Richardson number is increased. Maximum ratios between buoyancy and momentum fluxes (the flux Richardson number) appear to be limited by the value of .2 (Rohr et al., 1984) and thus the buoyancy flux is small 
enough to be ignored in the discussion which follows. In order to make the assertion that turbulent momentum fluxes alter the observed Richardson number distribution it needs to be demonstrated that the dissipation significantly affects the shear profile on the length scale which $\mathrm{N}^{2}$ and $\mathrm{S}^{2}$ are estimated. That calculation forms the basis for Section 4.5. It also needs to be shown that the time scale of the dissipative process is shorter than the fastest internal wave time scale so that mixing events have time to develop and that adiabatic processes do not conceal the effects of dissipation by themselves altering the $\mathrm{S}^{2}$ and $\mathrm{N}^{2}$ profiles over the mixing event time scale. We begin by considering the possible adiabatic internal wave time scales.

The time scale for nonlinear processes to flux energy to higher wavenumbers and increase the shear and strain variances at a given location can be simply calculated from the model of Henyey et al. From eq. 4.1: $\mathrm{dm} / \mathrm{dt}=-\mathbf{U}_{\mathrm{z}} \cdot \mathbf{k}_{\mathrm{h}}$. If representative values of $\mathrm{U}_{\mathrm{z}} \sim \mathrm{N}$ and $\mathrm{k}_{\mathrm{h}} \sim \mathrm{mf} / \mathrm{N}$ are chosen, then $\mathrm{T} \sim \mathrm{f}^{-1}$.

Estimates for the time between zeroes in a Gaussian wave field can be determined from the method of Rice (1944) and presented by Garrett and Munk (1972b). The time between zeroes is simply:

$$
\mathrm{T}=\pi\left\{\mathrm{M}_{\mathrm{o}}(\omega) / \mathrm{M}_{2}(\omega)\right\}^{1 / 2}, \mathrm{M}_{\mathrm{r}}(\omega)=\int \omega^{\mathrm{r}} \mathrm{F}(\omega) \mathrm{d} \omega,
$$

where $F(\omega)$ is the frequency spectral density of the wave field variable. At $\mathrm{N}=3 \mathrm{cph}$ for a $\mathrm{GM}$ wavefield the strain time scale is $2.8(\mathrm{Nf})^{-1 / 2}$. Since the strain pdf is not Gaussian this time scale is somewhat uncertain. Sherman and Pinkel (1991) argue that high N events have a longer correlation time due to the decrease of the strain in the larger scale internal wave field over the vertical extent 
of the high $\mathrm{N}$ event. The shear time scale is found to be $4.8(\mathrm{Nf})^{-1 / 2}$. We view the shear time scale as a potential underestimate. The shear components are $\pi / 2$ out of phase in general and of nearly equal amplitudes for near-inertial waves. For an internal wave field dominated by near-inertial frequencies the time scale for $S^{2}$ will be larger than that of $\mathrm{U}_{\mathrm{z}}$ or $\mathrm{V}_{\mathrm{z}}$ alone.

The time scale of the strain field does not necessarily imply a lower bound on the Richardson number time scale. Desaubies and Smith (1982) argue for a time scale between Richardson number events proportional to $\mathrm{N}^{-1}$ since in general a function of two independent variables will have a correlation time which is much shorter than that of the individual components. However, in a statistical simulation of a time dependent GM wavefield Bohrer (unpublished manuscript, 1983) found the average time between events with $\mathrm{Ri}<1 / 4$ lasting more than a buoyancy period was 3.5 hours at $\mathrm{N}=3 \mathrm{cph}$. Numerically this is equal to $7.5(\mathrm{Nf})^{-1 / 2}$ at $30^{\circ}$ latitude. From a neutrally buoyant float KWB find the average time between events to be approximately $5.5(\mathrm{Nf})^{-1 / 2}$. Since the shear variance is generally larger than the strain variance (the ratio for the GM spectrum is 3), the time scale of $\mathrm{Ri}$ events evidently reflects the longer time scale of the shear field and thus the assumption by Desaubies and Smith of a time scale proportional to $\mathrm{N}^{-1}$ seems inappropriate.

The linear internal wave time scale of foremost importance to the shear instability mechanism is the duration of supercritical Richardson number events. If the time scale for the event is larger than the duration time, little mixing will occur and the wave/wave 
interactions will flux energy upwavenumber adiabatically until a state is reached where the average supercritical Richardson number is sufficiently low to result in a large growth rate for shear instability. Estimates of the duration time of $\mathrm{Ri}<1 / 4$ events from KWB are approximately a buoyancy period: $2 \pi /\langle\mathrm{N}\rangle$, where $\langle\mathrm{N}\rangle$ is the ambient buoyancy frequency. They argue that the observed duration times are underestimates due to the internal wave field advecting low Richardson number events past their sensors in the vertical. At $\mathrm{N}=3 \mathrm{cph}$ and $30^{\circ} \mathrm{N}$, the observed duration time is only a factor of four larger than the strain time scale calculated above.

The time scale for the development of shear instability can be estimated from the linear instability analysis of Hazel (1972) and employed by KWB. For their data, KWB estimate an average growth time of $3 /<\mathrm{N}>$, half that of the observed duration time. For the local $6 \mathrm{~m}$ WRINCLE data an average value of the inverse time scale is found to be $.12<N>$. Similar values are estimated from the local $3 \mathrm{~m}$ FASINEX data $(.14<\mathrm{N}>)$. Thus the growth time scale for linear shear instability is approximately a buoyancy period for these data at these particular scales. At $\mathrm{N}=3 \mathrm{cph}$ and $30^{\circ} \mathrm{North}$ the linear instability time scale $(2 \pi / \mathrm{N})$ is numerically equal to $(\mathrm{Nf})^{-1 / 2}$ and thus the linear instability time scale is only marginally faster than the internal wave time scale. In a background flow field which does not start from a small amplitude state it is unclear how many time scales would be required for the instability to develop into a mixing event. The observations of KWB imply a minimum of two instability time scales. 
A dissipation time scale can also be estimated from the $6 \mathrm{~m}$ WRINCLE data. The KWB model can be expressed as $\varepsilon=\delta \mathrm{KE} / \delta \mathrm{T}$ where

$$
\delta \mathrm{KE}=\frac{1}{24} \Delta \mathrm{z}^{2} \mathrm{~N}^{2}\left(\frac{1}{\mathrm{Ri}}-\frac{1}{\mathrm{Ri}_{\mathrm{c}}}\right) .
$$

The half-decibar spaced dissipation estimates were first averaged over the $6 \mathrm{~m}$ window of the $\mathrm{S}^{2}$ and $\mathrm{N}^{2}$ estimates and the resulting estimates divided by the corresponding local estimates of $\delta \mathrm{KE}$ and then averaged. An average time scale of $\langle\varepsilon / \delta \mathrm{KE}\rangle^{-1}=.82 / \mathrm{N}$ and $2.9 / \mathrm{N}$ was obtained for $\mathrm{Ri}_{\mathrm{c}}=1 / 4$ and $1 / 3$, respectively. The time scale for dissipation is observed to be small compared to the adiabatic internal wave time scales: Once mixing is initiated, it proceeds to quickly modify the background shear field. The adiabatic time scales are too large to obscure dissipative effects.

We therefore propose the following physical interpretation of the statistical dependance between $\mathrm{N}^{2}$ and $\mathrm{S}^{2}$. As Ri decreases below some critical point in a time dependant field, instability develops and ultimately results in dissipation (Figure 4.4). Associated with the dissipation are, from production/dissipation balances, momentum and buoyancy fluxes. We infer from observations that the time scale to dissipate the estimated available kinetic energy is small - i.e. proportional to $\mathrm{N}^{-1}$ in magnitude, and notably smaller than any other time scale in the internal wave field. Thus the momentum fluxes result in a significant reduction of the shear so that the Richardson number is fluxed to a larger value. This adjustment occurs on a time scale much shorter than the internal wave field can 'reset' the Richardson number, either by waves propagating through and 
terminating the supercritical event or by nonlinear wave-wave interactions transporting energy upscale. Both linear propagation and nonlinear wave/wave interactions occur on the time scale of the wave period, which is influenced by near-inertial content. We are able to observe a dependance between $\mathrm{N}^{2}$ and $\mathrm{S}^{2}$ in the FASINEX data because their diffusive signatures in the shear and strain field are altered only over rather large time scales. This process is depicted in Figure 4.16.

Given the diffusive interpretation of the $\mathrm{N}^{2}-\mathrm{S}^{2}$ dependance the percentage of $\mathrm{Ri}<1 / 4$ is expected to be a function of $\mathrm{N}$ even though $<\mathrm{S}^{2}>/ \mathrm{N}^{2}$ is independent of depth when calculated over a depth scale inversely proportional to the cutoff wavenumber. At very low $\mathrm{N}$, the time scale for the shear instability process to generate turbulent fluxes becomes similar to the internal wave propagation time scale, and thus the wavefield resets the $\mathrm{Ri}$ distribution, terminating supercritical events before the shear instability process results in turbulent mixing. The wave/wave interactions act on a somewhat slower time scale. Their effect is to flux energy upwavenumber and concentrate shear at smaller scales, where the Richardson number approaches sufficiently low values that the shear instability time scale is larger than the adiabatic propagation time scales. The vertical scale over which the turbulent momentum fluxes act is thus smaller for smaller N. Consequently the statistical dependence between $\mathrm{N}^{2}$ and $\mathrm{S}^{2}$ is predicted to exhibit some buoyancy scaling. Larger percentages of $\mathrm{Ri}<1 / 4$ at low $\mathrm{N}$ and lower values of the Richardson number are anticipated as the separation between the 


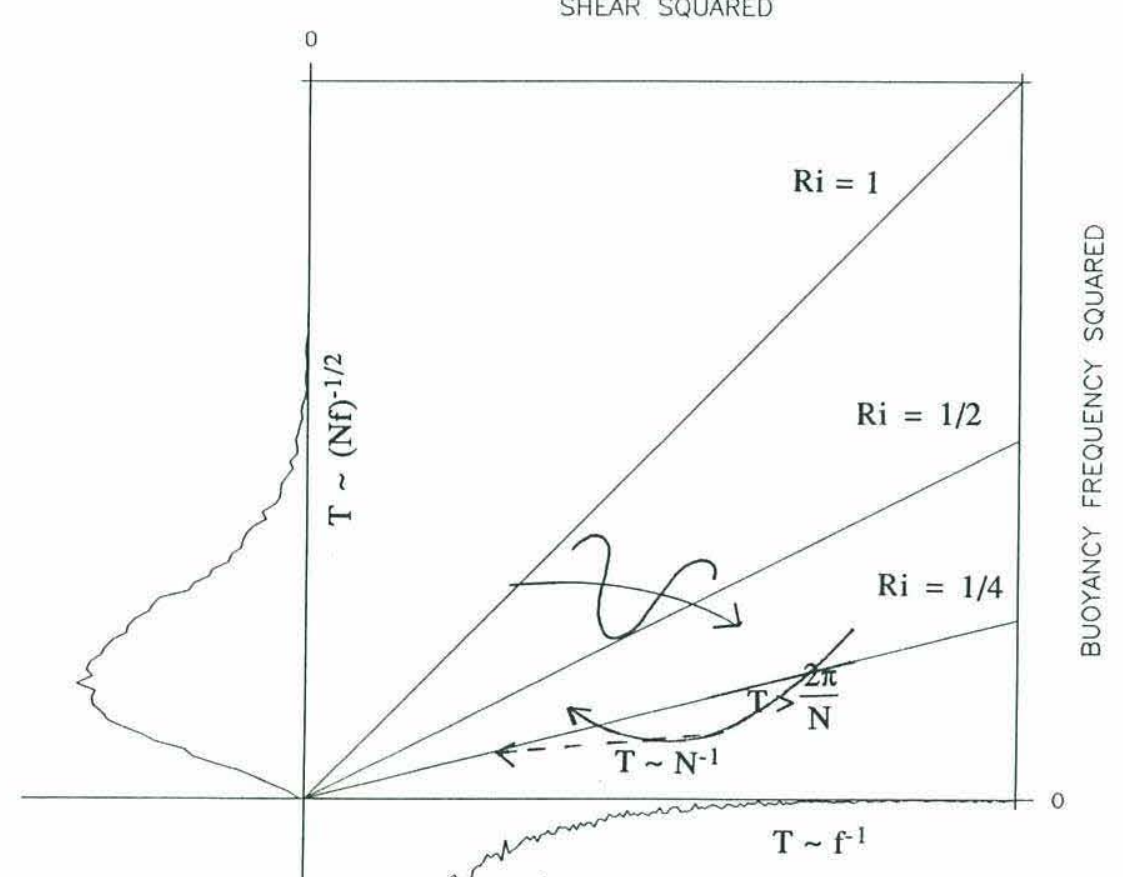

Figure 4.16: A schematic interpretation of figure 4.4 and 4.15. The time scale of internal wave motions projected along the $\mathrm{N}^{2}$ axis is $\mathrm{O}\left((\mathrm{Nf})^{-1 / 2}\right)$ and along the $\mathrm{S}^{2}$ axis $\left(\mathrm{O}\left(\mathrm{f}^{-1}\right)\right)$. For the $\mathrm{GM}$ spectrum the $\mathrm{N}^{2}$ variance is $1 / 3$ the shear variance and trajectories in the plot have smaller vertical excursions. A parcel of water follows some complicated trajectory in $\mathrm{N}^{2}-\mathrm{S}^{2}$ space. When $\mathrm{Ri}<1 / 4$ growth of unstable waves is predicted. The apparent time scale required for waves to break is $>2 \pi / \mathrm{N}$ (see text). If supercritical Richardson number events are not terminated by wave propagation effects, significant fluxes of momentum and buoyancy occur which alter the Richardson number over a time scale short $\left(\mathrm{O}\left(\mathrm{N}^{-1}\right)\right)$ in comparison to internal wave motions. The result is a decrease in the frequency of occurrence of low Richardson number events and a statistical dependence between $\mathrm{N}^{2}$ and $\mathrm{S}^{2}$. 
time scale of the shear instability mechanism and the adiabatic internal wave propagation time scale becomes smaller.

These expectations are born out by the TOPO_DEEP stations, Figures 4.17 and 4.18. In Figure 4.17, the observed (local) percentage of $\mathrm{Ri}<1 / 4$ and the percentage of $\mathrm{Ri}<1 / 4$ based upon the randomly sampled pdf versus $\mathrm{N}^{2}$ are displayed. Relevant parameters appear in Table 4.1. The lowest $\mathrm{N}$ value has the highest percentage of low $\mathrm{Ri}$ for the local data. The estimates from the randomly sampled pdf do not reveal a trend. As $\mathrm{N}$ decreases the difference between the two estimates diminishes. We thus conclude that the observed statistical dependance between $\mathrm{N}^{2}$ and $\mathrm{S}^{2}$ results in the difference between the estimates and that the statistical dependance between $\mathrm{N}^{2}$ and $\mathrm{S}^{2}$ exhibits some buoyancy scaling. In Figure 4.18, the randomly sampled pdf is used to produce dissipation estimates from KWB's model. Unlike the random data, the randomly sampled pdf does not collapse the observed dissipation estimates. Compared with Figure 4.6, the randomly sampled pdf reveals a consistent trend with $\mathrm{N}$. This trend is of about an order of magnitude in size and is comparable to that if the nominal scaling of $\mathrm{E}^{2} \mathrm{~N}^{3}$ applied. The $\mathrm{N}^{2}-\mathrm{S}^{2}$ dependence is interpreted as resulting from the effects of dissipation. We interpret the buoyancy scaling of the $\mathrm{N}^{2}-\mathrm{S}^{2}$ dependence to be due to the decrease in scale separation between the time scale of the shear instability mechanism and the adiabatic internal wave time scale. Despite the apparently small numerical range of the ratio between shear instability event and internal wave time scales over the TOPO_DEEP data set $\left((\mathrm{N} / \mathrm{f})^{1 / 2}\right.$ varies by a factor of 3.5$)$, this difference is sufficient to impact the Richardson number statistics. 


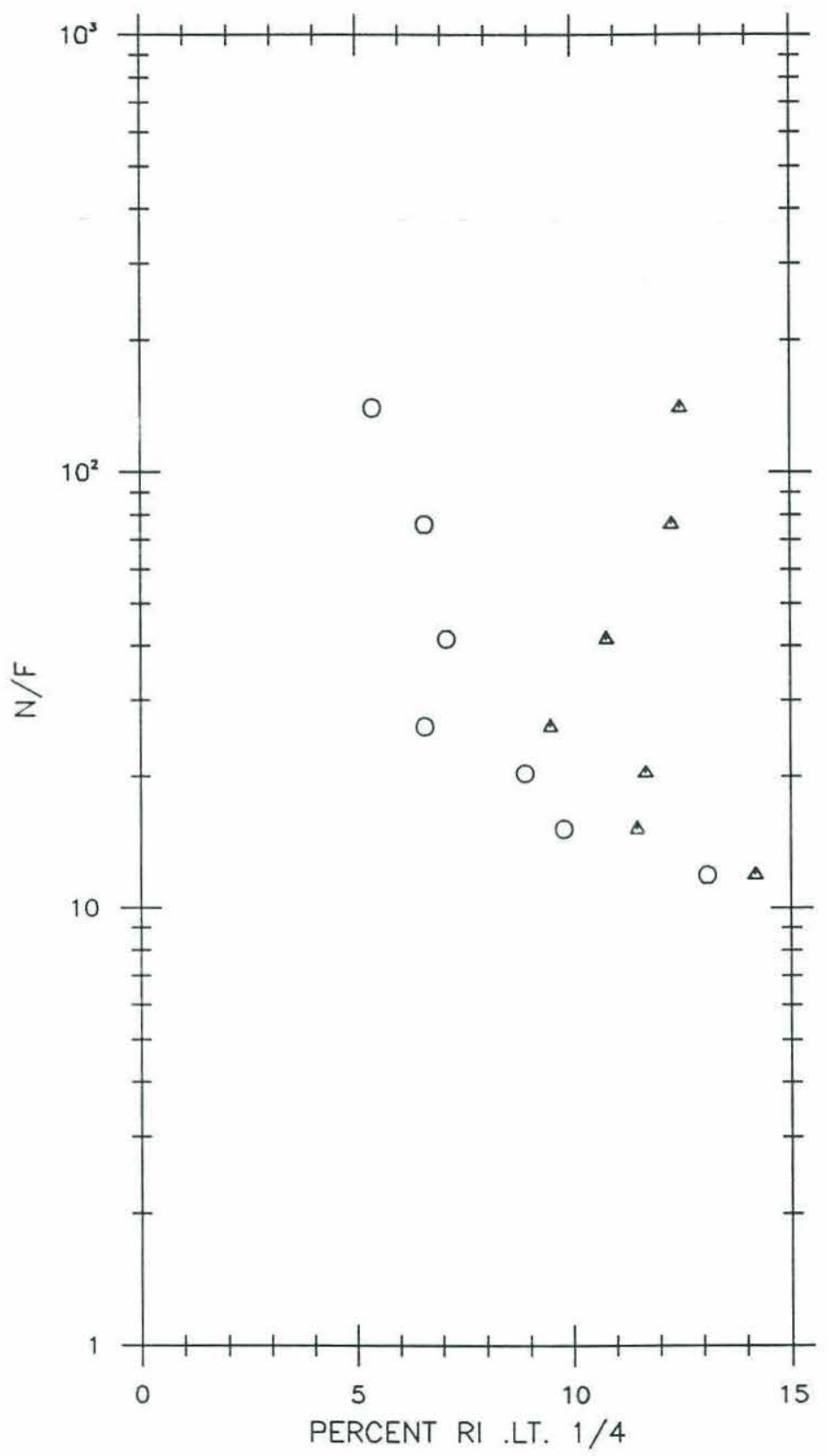

Figure 4.17: The percentage of observed (local) $\mathrm{Ri}<1 / 4$ (o) and the percentage of $\mathrm{Ri}<1 / 4$ from the randomly sampled pdf $(\Delta)$ versus $\mathrm{N} / \mathrm{f}$ for the TOPO_DEEP data (Table 4.1). The two estimates become similar at low $\mathrm{N}$ due to the lack of a scale separation between the shear instability mechanism and the time scale for wave propagation effects to terminate the supercritical event before the event 'breaks'. 


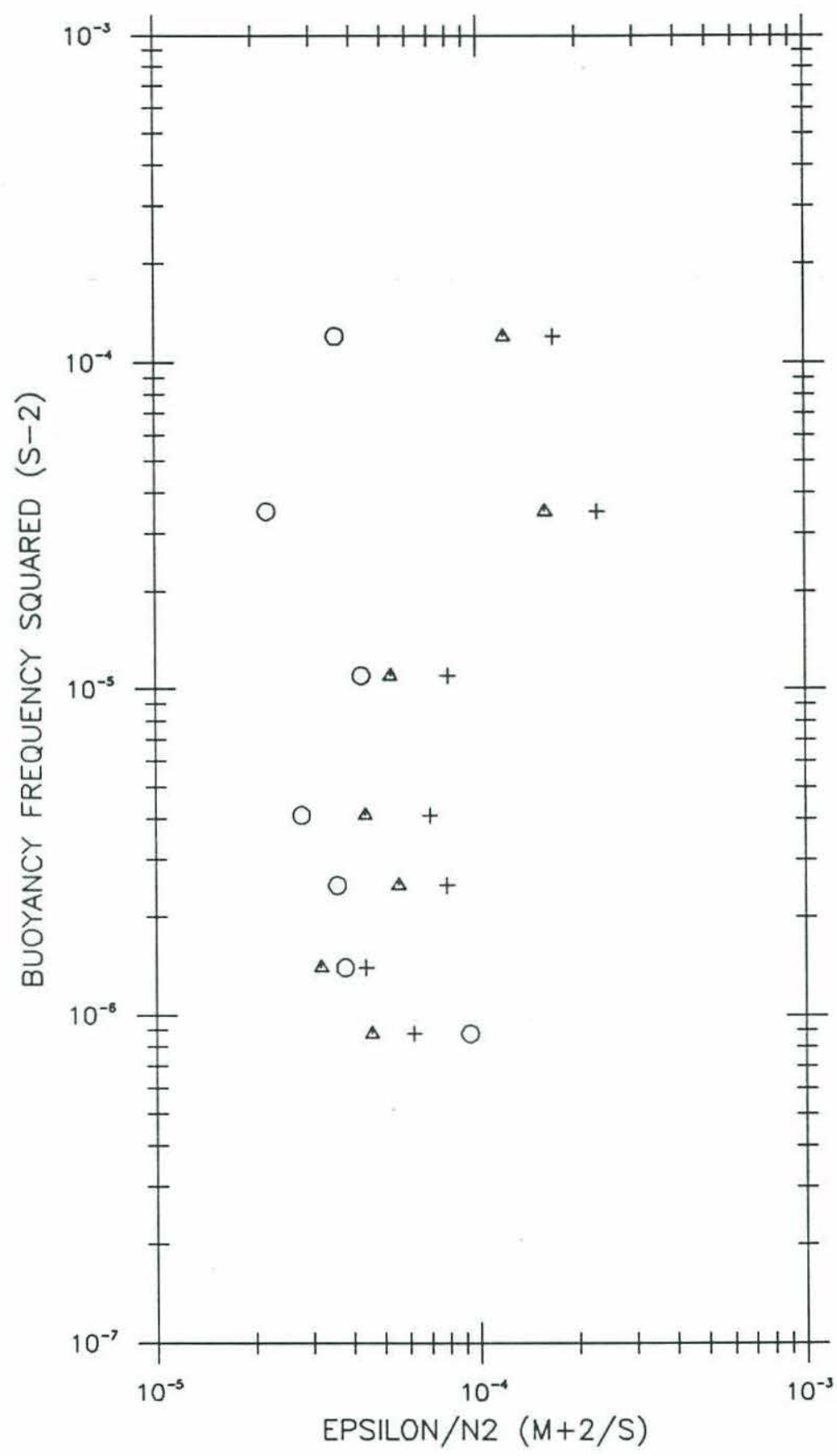

Figure 4.18: Dissipation estimates from the model of KWB using a randomly sample pdf for the TOPO_DEEP data $\left(\Delta\right.$ implies $\operatorname{Ri}_{\mathrm{c}}=1 / 4$, + implies $\left.\mathrm{Ri}_{\mathrm{c}}=1 / 3\right)$. Observed dissipation rates are plotted as circles (o). Compare with Figure 4.6. The dissipation estimates are not collapsed by the randomly sampled pdf. The mixed estimates exhibit a trend of approximately an order of magnitude. 


\subsection{Dynamics of the transition region $\mathrm{m}_{\mathrm{c}}<\mathrm{m}<\left(2 \pi \mathrm{L}_{\mathrm{o}}\right)^{-1}$}

The question addressed by researchers in the context of saturated spectral theory is whether linear instability mechanisms can control the amplitude of a wave packet as that wave packet is fluxed to higher wavenumbers. The wave packet is assumed to be limited to amplitudes which produce conditions marginally exceeding the linear instability criterion for a portion of the profile. If a wave packet has a wavelength of $2 \pi / \mathrm{m}$ and envelope size $\mathrm{O} \sim(1 / \mathrm{m})$, the spectral density of the wave packet will be concentrated in a region $\pm m / 2$. A boxcar average of the spectral density produces the average shear variance in the packet:

$$
<\mathrm{S}^{2}>=\mathrm{mS}_{\mathrm{z}}
$$

The amplitude criterion for a wave packet is obtained by considering the vertical profile for a single plane internal wave. The two mechanisms most often considered are shear and advective instability. The advective instability criterion $\mathrm{U} / \mathrm{c}>1$ implies for a single plane wave that the buoyancy profile will be convectively unstable over some portion of the profile (Orlanski and Bryan, 1969). On the basis of work from Fritts and Rastogi (1985) and Fritts and Yuan (1989), KWB assert that shear instability is more likely for a single linear plane wave with a frequency $<3 f$ than advective instability and that the two criteria require similar amplitudes for a single wave with a frequency $>3 \mathrm{f}$, with the caveat that phase speeds and the frequency 'responsible' for instability are ill defined in a stochastic internal wave field. The dependence on frequency content arises as near inertial waves have small density perturbations relative to their 
shear and are thus not as likely to overturn as high frequency waves. The average frequency for the GM spectrum from the shear/strain ratio is approximately $\sqrt{2} \mathrm{f}$ and implies the shear instability mechanism to be more likely than advective instability in the oceanic internal wave field. This contention is supported by KWB's observation that supercritical Richardson number events are associated with lower average intrinsic frequencies and the absence of overturning events in their data. Kunze et al. present the single wave amplitude criterion and two examples of Richardson number profiles $(\omega / \mathrm{f}=1.05$ and 10.0) in their Figures 2 and 1, respectively. These are reproduced in our Figure 4.19. For the high frequency case the condition of marginal stability is $\max \left|\mathrm{V}_{\mathrm{z}}\right| \cong 1.0 \mathrm{~N}$. The corresponding shear variance is $[1.0 \mathrm{~N}]^{2} / 2$. Since the shear variance is constrained to one component for a high frequency wave, $\left\langle\mathrm{S}^{2}\right\rangle=\mathrm{N}^{2} / 2$. Equating this with the wave packet criterion produces $\mathrm{mS}_{\mathrm{z}}=\mathrm{N}^{2} / 2$. This is exactly the criterion in Munk (1981) for the cutoff wavenumber which we have utilized extensively. For the near-inertial internal wave the amplitude criterion of $\max \left|\mathrm{V}_{\mathrm{z}}\right| \cong 1.6 \mathrm{~N}$ produces an estimate of $\left\langle\mathrm{S}^{2}\right\rangle=\left\langle\mathrm{U}_{\mathrm{z}}^{2}\right\rangle+\left\langle\mathrm{V}_{\mathrm{z}}^{2}\right\rangle=[1.6 \mathrm{~N}]^{2} / 2 .+[1.6 \mathrm{~N}]^{2} / 2 .=2.56 \mathrm{~N}^{2}$, a factor of five larger than the high frequency amplitude criterion. Since the oceanic internal wave field is predominantly near-inertial, the wavepacket theory would seem to predict a higher saturated spectral level than is observed in the ocean. Similar results are found for the atmospheric internal wave field. The atmospheric internal wave field is generally dominated by continuum band frequencies $(\omega>3 f)$ and so the theoretical criterion of $\mathrm{mS}_{\mathrm{z}}=\mathrm{N}^{2} / 2$ is taken as applicable. 


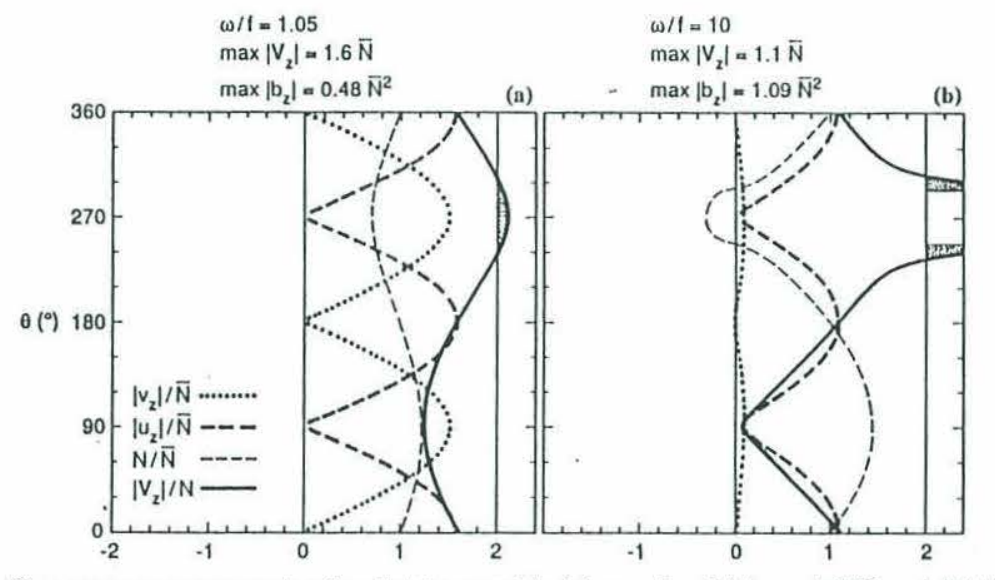

Fig. 1. Profiles over one wave cycle of a slightly unstable $(a)$ near-inertial $(\omega=1.05 f)$ and $(b)$ high-frequency $(\omega=10 f)$ wave. Curves include the two shear components normalized by the unperturbed background buoyancy requency $N$ (thick dashed and thick dotted curves), the Froude nimber $V_{2} / N$ (thick solid curve), and the buoyancy

Froude number $\left(V_{2} / N>2\right)$ are stippled. These are associated with the phase of weakest stratification.

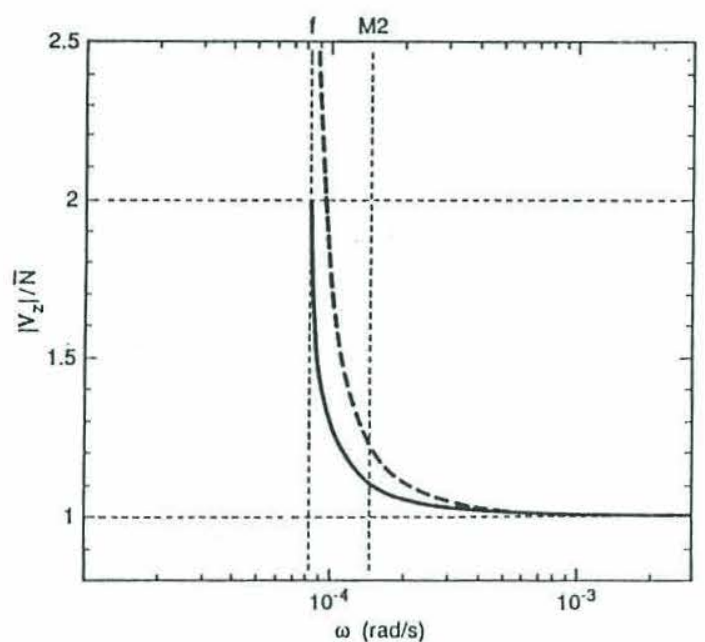

Fig. 2. The marginally unstable wave amplitude $\max \left|\mathbf{V}_{z}\right| / N$ for shear (solid) and buoyancy (dashed) instabilities as a function of frequency. Instibility of either kind requires larger amplitudes for near-inertial waves. For near-inertial waves, shear instability occurs at smaller amplitudes than buoyancy instability. For high-frequency $(\omega>3 f)$ waves, he marginally sta

Figure 4.19: Figure 1 and 2 from KWB. 
Observed spectral values result in approximately $\mathrm{mS}_{\mathrm{z}}=\mathrm{N}^{2} / 6$ (Smith et al., 1987), a factor of 3 below the theoretical value.

The difference between the theoretical and observed values is generally ascribed to the wavepacket scheme not being an adequate representation of a strongly nonlinear, stochastic wave field. Proponents of the saturated spectral theories (e.g. Fritts (1989), Smith et al. (1987), Dewan and Good (1986)) suggest that the difference between the wave packet derived criteria and the observations is largely due to the amplitude criterion of the wave packet representation being an inadequate representation of the statistical properties of instability mechanisms in a stochastic wavefield (e.g. Section 4.4). A simple argument has been forwarded to address the difference and a criterion of $\mathrm{mS}_{\mathrm{z}}=\mathrm{N}^{2} / 6$ results (Smith et al., 1987). Critics of the saturated theories (e.g. Hines (1991d), Dunkerton (1989)) focus on the nonlinear aspects of the problem to produce adiabatic criteria.

Hines (1991a,b,c 1989) argues that the predominant nonlinearity is contained in the Doppler shift of small scale internal waves by internal waves of larger scale and proceeds to determine the criterion of $\mathrm{mS}_{\mathrm{z}}=\mathrm{N}^{2} / \pi$ from an analytical statistical method. Rotational effects were not included in that model. The ray tracing model of HWF, in which the interaction is entirely in the Doppler shift, also appears to be in equilibrium with respect to an $\mathrm{m}^{-1}$ shear spectrum for $\mathrm{m}_{\mathrm{c}}<\mathrm{m}<2 \mathrm{~m}_{\mathrm{c}}$ using the Munk (1981) version of the GM spectrum. This results in a criterion of $\mathrm{mS}_{\mathrm{Z}}=\mathrm{N}^{2} / 2$. We pursue a discussion in terms of HWF as it proves to be more physically enlightening.

From HWF the dissipation can be equated with the time rate of change of energy in the internal wave field, $\varepsilon=\mathrm{dE} / \mathrm{dt}$. This equality 
assumes that the wavenumber and frequency spectral shapes are statistically stationary and thus $\varepsilon$ is interpreted as the average upwavenumber energy flux and is independent of vertical wavenumber. This equality can be rewritten as

$$
\varepsilon=\left\langle\frac{\mathrm{dE}}{\mathrm{dm}} \frac{\mathrm{d} \mathrm{m}}{\mathrm{dt}}>\right.
$$

where $\mathrm{dE} / \mathrm{dm}$ is the energy spectral density and $\mathrm{dm} / \mathrm{dt}$ is the rate of change of vertical wavenumber. Henyey et al. find that the dominant balance for the time dependence of the vertical wavenumber is $\mathrm{dm} / \mathrm{dt}=-\mathbf{U}_{\mathrm{z}} \cdot \mathbf{k}_{\mathrm{h}}$ where $\mathbf{k}_{\mathrm{h}}$ is the horizontal wavenumber of the (small scale) test wave and $\mathbf{U}_{\mathrm{z}}$ is the vertical shear of the (large scale) background. In general one can write

$$
\varepsilon=\frac{\mathrm{dE}}{\mathrm{d} \mathrm{m}}\left(\mathrm{U}_{\mathrm{z}}^{2}\right)^{1 / 2} \mathrm{k}_{\mathrm{h}} \mathrm{C}(\mathrm{m}),
$$

where $\mathrm{C}(\mathrm{m})$ represents the correlation coefficient between $\mathbf{U}_{\mathrm{z}}$ and $\mathbf{k}_{\mathrm{h}}$ and provides a measure for assessing the effectiveness of the wave/wave interactions at producing a net upwavenumber energy flux.. Henyey et al. employed a Monte-Carlo approach to determine $\mathrm{C}(\mathrm{m})$ for test waves propagating in a GM internal wave background. From $\varepsilon=(\mathrm{dE} / \mathrm{dm})(\mathrm{dm} / \mathrm{dt})$ we note that a change in the vertical wavenumber dependence of $\mathrm{dE} / \mathrm{dm}$ at $\mathrm{m}_{\mathrm{c}}$ from $\mathrm{m}^{-2}$ to $\mathrm{m}^{-3}$ requires a corresponding increase in the wavenumber dependence of the upwavenumber flux rate, $\mathrm{dm} / \mathrm{dt}$. This increase is apparently related to the increasing effectiveness of the ability of small scale waves to interact with large scale waves through the Doppler shift. Since $\left(\mathrm{U}_{\mathrm{z}}^{2}\right)^{1 / 2}$ can be written as $\left(\mathrm{mS}_{\mathrm{z}}\right)^{1 / 2}$ or $\left(\mathrm{m}^{3}(\mathrm{dE} / \mathrm{dm})\right)^{1 / 2}$ for $\mathrm{m}<\mathrm{m}_{\mathrm{c}}$ and 
$\left(m_{c} S_{z}\left(1+\ln \left(m / m_{c}\right)\right)^{1 / 2}\right.$ for $m>m_{c}$, it is apparent that if $k_{h}$ is written as $\gamma(\mathrm{m}) \mathrm{mf} / \mathrm{N}$ (i.e. assuming a near-inertial frequency content of the wavefield), where $\gamma(\mathrm{m})$ is taken to represent a dependence of frequency content of the wave field upon vertical wavenumber, then

$$
\begin{aligned}
\varepsilon & \sim \mathrm{m}^{-1 / 2} \mathrm{C}(\mathrm{m}) \gamma(\mathrm{m}) \text { for } \mathrm{m}<\mathrm{m}_{\mathrm{c}} \text { and } \\
\varepsilon & \sim \mathrm{m}^{-2} \mathrm{C}(\mathrm{m}) \gamma(\mathrm{m}) \text { for } \mathrm{m}>\mathrm{m}_{\mathrm{c}} .
\end{aligned}
$$

If the spectral shapes are statistically stationary, $\varepsilon$ is independent of $\mathrm{m}$ and therefore $\mathrm{C}(\mathrm{m}) \gamma(\mathrm{m})$ scales as $\mathrm{m}^{1 / 2}$ for $\mathrm{m}<\mathrm{m}_{\mathrm{c}}$ and as $\mathrm{m}^{2}$ for $\mathrm{m}>$ $\mathrm{m}_{\mathrm{c}}$. In the HWF model, both $\mathrm{C}(\mathrm{m})$ and $\gamma(\mathrm{m})$ are increasing: upfrequency fluxes are noted, implying increasing horizontal wavenumber content at smaller scales. A dramatic increase in the effectiveness of the Doppler shifting interaction at the cutoff wavenumber, $\mathrm{m}_{\mathrm{c}}$ is implied.

Since both the saturated spectral arguments and the Doppler shifting theories result in similar amplitudes and slopes for the atmospheric internal wave shear spectrum they cannot be distinguished on this basis. Differentiating between the two approaches appears to require defining the vertical scale over which the turbulent momentum fluxes affect the shear profiles and assessing the relative impact as a function of wavenumber.

In Section 4.4 the statistical dependence between $\mathrm{N}^{2}$ and $\mathrm{S}^{2}$ resulted in a significant loss of shear variance associated with supercritical Richardson number events for the 3m FASINEX data. However, since only $5 \%$ of the randomly sampled data was noted to be supercritical, one questions whether this would have a significant impact upon the shear spectrum. 
That question is addressed by estimating the fraction of the shear variance to be dissipated with respect to the variance contained in the portion of the spectrum $m>m_{c}$, where $m_{c}$ is defined by

$$
\int_{0}^{m_{c}} S_{z} d m=N^{2} / 2 .
$$

This assumption will be examined shortly. The randomly sampled FASINEX values are used for this calculation as the observed local values exhibit significant changes due to the effects of turbulent momentum fluxes (Section 4.4). Relevant parameters are to be found in Table 4.2. The fraction of the shear variance to be dissipated is calculated by determining the average value of the excess shear variance which results in a supercritical value of the Richardson number $\left(\mathrm{S}^{2}-\mathrm{N}^{2} / \mathrm{Ri}_{\mathrm{c}}\right)$, multiplying by the fraction (fr) of observed events to obtain the total dissipated variance, and then dividing by the variance in the spectrum corresponding to wavenumbers $\mathrm{m}_{\mathrm{c}}<\mathrm{m}$ $<\mathrm{m}^{\prime},\left\langle\mathrm{S}_{*}^{2}>\left(<\mathrm{S}^{2}>=\mathrm{N}^{2}\left(1+\ln \left(\mathrm{m}^{\prime} / \mathrm{m}_{\mathrm{c}}\right)\right) / 2\right.\right.$ and $\left\langle\mathrm{S}_{*}^{2}>=\mathrm{N}^{2} \ln \left(\mathrm{m}^{\prime} / \mathrm{m}_{\mathrm{c}}\right) / 2\right)$. The question we next pose is: If the fluxes are assumed to act upon wavenumbers $\mathrm{m}>\mathrm{m}_{\mathrm{c}}$, what is the resulting spectral slope if a power law dependance (i.e. $\mathrm{S}_{\mathrm{z}}=\mathrm{A}\left(\mathrm{m} / \mathrm{m}_{\mathrm{c}}\right)^{-\mathrm{p}}$ ) is assumed? To find the slope we attempt to solve:

$$
\int_{m_{c}}^{m^{\prime}}\left(\frac{m}{m_{c}}\right)^{-p} d m=\left(1-\frac{\left.f r<S^{2}-N^{2} / R i_{c}\right\rangle}{\left.<S_{*}^{2}\right\rangle}\right) \int_{m_{c}}^{m^{\prime}}\left(\frac{m}{m_{c}}\right)^{-1} d m,
$$

which reduces to 


$$
\frac{1}{1-\mathrm{p}}\left(\left(\frac{\mathrm{m}^{\prime}}{\mathrm{m}_{\mathrm{c}}}\right)^{1-\mathrm{p}}-1\right)=\left(1-\frac{\mathrm{fr}\left\langle\mathrm{S}^{2}-\mathrm{N}^{2} / \mathrm{Ri}_{\mathrm{c}}\right\rangle}{\left\langle\mathrm{S}_{*}^{2}\right\rangle}\right) \ln \left(\frac{\mathrm{m}^{\prime}}{\mathrm{m}_{\mathrm{c}}}\right)
$$

For $\mathrm{Ri}_{\mathrm{c}}=1 / 4$ and the values in Table 4.2 we find by trial and error $\mathrm{p} \sim 3 / 2$ for the 2 and $3 \mathrm{~m}$ data. For $\mathrm{Ri}_{\mathrm{c}}=1 / 3$ we find a value of $1.7<$ $\mathrm{p}<2.0$. We therefore conclude that the shear instability mechanism may significantly affect the spectral slope for wavenumber between $\mathrm{m}_{\mathrm{c}}$ and $2 \mathrm{~m}_{\mathrm{c}}$. We have assumed implicitly here that waves on scales $\mathrm{m}<\mathrm{m}_{\mathrm{c}}$ will not be strongly influenced. While this seems to be reasonable due to the absence of supercritical events, this can be verified with the $7 \mathrm{~m}$ data. If one assumes that $\left\langle\mathrm{S}_{*}^{2}\right\rangle=\mathrm{N}^{2} / 4$, then from Table 4.2 only $2 \%$ and $3 \%$ of the total shear variance in the wavenumber region $\mathrm{m}_{\mathrm{c}} / 2<\mathrm{m}<\mathrm{m}_{\mathrm{c}}$ is dissipated the criteria $\mathrm{Ri}_{\mathrm{c}}=1 / 4$ and $1 / 3$, respectively.

Table 4.2: Parameters for the saturated spectral calculations (eq. 4.7). For $\delta=7 \mathrm{~m},\left\langle\mathrm{~S}_{*}^{2}\right\rangle=\mathrm{N}^{2} / 4$ has been assumed.

$\delta$ $2 \mathrm{~m}$

$3 \mathrm{~m}$ $7 \mathrm{~m}$

\begin{tabular}{l|cc|cc|cc}
$\mathrm{Ri}$ criterion $\left(\mathrm{Ri}_{\mathrm{c}}\right)$ & $1 / 4$ & $1 / 3$ & $1 / 4$ & $1 / 3$ & $1 / 4$ & $1 / 3$ \\
\hline $\mathrm{S}^{2}>/ \mathrm{N}^{2}$ & .92 & .92 & .79 & .79 & .51 & .51 \\
$<\mathrm{S}^{2}-\mathrm{N}^{2} / \mathrm{Ri}_{\mathrm{c}}>/<\mathrm{S}^{2}>$ & .97 & .99 & .96 & .97 & 1.01 & 1.00 \\
$\% \mathrm{Ri}<\mathrm{Ri}_{\mathrm{c}} \quad(\mathrm{fr})$ & .081 & .121 & .051 & .082 & .008 & .016 \\
$\mathrm{~m}^{\prime} / \mathrm{m}_{\mathrm{c}}$ & 2.32 & 2.32 & 1.79 & 1.79 & 1.02 & 1.02 \\
$\left.\mathrm{fr}<\mathrm{S}^{2}-\mathrm{N}^{2} / \mathrm{Ri}_{\mathrm{c}}>/<\mathrm{S}_{*}^{2}\right\rangle$ & .17 & .26 & .13 & .22 & .02 & .03
\end{tabular}

This success prompts us to investigate the effects on a spectrum which is white until the point $\left\langle\mathrm{S}^{2}\right\rangle=\mathrm{N}^{2}$. Assuming that $\left.\left.<\mathrm{S}^{2}-\mathrm{N}^{2} / \mathrm{Ri}_{\mathrm{c}}\right\rangle /<\mathrm{S}^{2}\right\rangle \sim 1.0$ is a general result from the data presented above and reasonable values for fr of .10 and .15 for $\mathrm{Ri}_{\mathrm{c}}=1 / 4$ and $1 / 3$ (Figure 4.17), respectively, then the fractional decrease of 
variance in the wavenumber band for which $\mathrm{N}^{2} / 2<\left\langle\mathrm{S}^{2}\right\rangle<\mathrm{N}^{2}$ is found to be .20 and .30 , respectively. The $30 \%$ decrease leads to a -1 spectrum as $\ln (2)=.69$. It is therefore reasonable to conclude that the spectrum is potentially saturated for wavenumbers as small as the observed cutoff. In what follows we refer to the decrease in the shear spectrum related to the influence of turbulent momentum fluxes as a diffusive cutoff. This cutoff need not be equal to $\mathrm{m}_{\mathrm{c}}$. We infer from the above example that the diffusive cutoff occurs at a shear variance $.5 \mathrm{~N}^{2}<<\mathrm{S}^{2}><.7 \mathrm{~N}^{2}$.

An $\mathrm{m}^{-1}$ shear spectrum is not to be viewed as a direct consequence of this calculation. The observed oceanic spectrum will represent the effects of dissipation upon the adiabatic spectrum. If the adiabatic and diffusive cutoffs are similar and the effects of wave/wave interaction lead to a -1 slope, diffusive effects may reduce the slope further. We suspect that the adiabatic cutoff may occur at a lower wavenumber for an internal wavefield with higher frequency content. The adiabatic cutoff in the HWF spectrum is associated with an abrupt transition in the effectiveness of the Doppler shift mechanism and is associated with an increase in the frequency content of the test wave spectrum. In that model higher frequency waves have larger interaction rates and a test wave spectrum of higher frequency content, being more sensitive to Doppler shifting, may possibly exhibit a lower transition wavenumber. This appears to be reasonable in view of the smaller observed atmospheric cutoffs $\left(\mathrm{mS}_{\mathrm{z}}=\mathrm{N}^{2} / 6\right)$ and the higher frequency content of that wave field. Sherman and Pinkel (1991) find an opposite trend in the frequency/vertical wavenumber dependence of the small-scale 
strain field in Eulerian and semi-Lagrangian (isopycnal following) reference frames. They do suggest, however, that in a truly Lagrangian frame the high frequency portion of the high vertical wavenumber spectrum may exhibit smaller cutoffs than that of the low frequency portion. They argue that at a given vertical wavenumber, high frequency waves are more susceptible to encountering critical layers, $U_{0}-c=U_{0}-\left(N \omega / m\left(\omega^{2}-f^{2}\right)^{1 / 2}\right)= \pm f$.

The diffusive cutoff wavenumber may also exhibit some frequency dependance. In Section 4.4 it was determined that the $\mathrm{N}^{2}$ variability played a significant role in determining the frequency of occurrence of supercritical Richardson number events. Since the shear/strain ratio is related to frequency content (Chapter Three, eq. 3.10), higher frequency content would result in the increased occurrence of instability at a given length scale and thus a smaller cutoff wavenumber.

Implicit in this calculation is the assumption that the internal wave field represents a steady profile upon which the turbulent momentum fluxes act. In a linear internal wave field a new Richardson number profile will be presented on a time scale $\left(\mathrm{T} \sim \mathrm{O}(\mathrm{Nf})^{-1 / 2}\right.$ ) and the turbulent momentum fluxes produced by shear instability will act to decrease the shear spectrum further. Conversely, if there is no scale separation between the growth rates $(\mathrm{T} \sim 2 \pi / \mathrm{N})$ and the rate at which the internal wave field evolves due to linear dynamics $\left(\mathrm{T} \sim \mathrm{O}(\mathrm{Nf})^{-1 / 2}\right)$, individual supercritical Richardson number events will be terminated before they result in turbulence, nonlinear processes will transfer energy upwavenumber on a time scale $\left(\mathrm{T} \sim \mathrm{O}\left(\mathrm{f}^{-1}\right)\right)$ and the turbulent momentum fluxes will act upon 
smaller scales where the rms shear is large enough to establish larger shear instability growth rates. In the limit of very small $\mathrm{N} / \mathrm{f}$ (e.g $4 \pi$ ), the linear instability growth rates may not be sufficiently large to establish shear instability as an intermediate mechanism linking the wave/wave interactions to turbulence. The relevant time scales thus appears to be related to $\mathrm{N}$ for the shear instability process and the frequency content of the internal wave field, which results in both the linear time scale for the internal wave field to evolve and the time scale for nonlinear interactions.

At low $\mathrm{N}$ the diffusive cutoff appears to be larger than the upper limit of integration which results in $\left\langle\mathrm{S}^{2}\right\rangle=1.1-1.2 \mathrm{~N}^{2}$. For TOPO_D7 and D6 the ratio N/f is approximately 12 and 15, respectively. If the shear instability time scale is $2 \pi / \mathrm{N}$, there is no scale separation between the linear instability time scale, the linear propagation time scale and the corresponding time scale for wavewave interactions to flux energy to higher wavenumbers. There is correspondingly virtually no reduction in the $\%$ of local $\mathrm{Ri}<1 / 4$ with respect to the randomly sampled data. Figure 4.20 presents the TOPO_D2-D7 spectra. These spectra also appear in Figure 3.5, Chapter Three and are described more completely there. The spectrum with the highest buoyancy frequency (D2) is denoted by the thick line lies below the spectra for the lower buoyancy frequencies, consistent with the saturated spectral theories. However, those spectra are more remarkable for the degree to which they are collapsed by the canonical scaling.

The second variable is the frequency content of the internal wave field. Figure 4.21 presents the WRINCLE (lower than average 


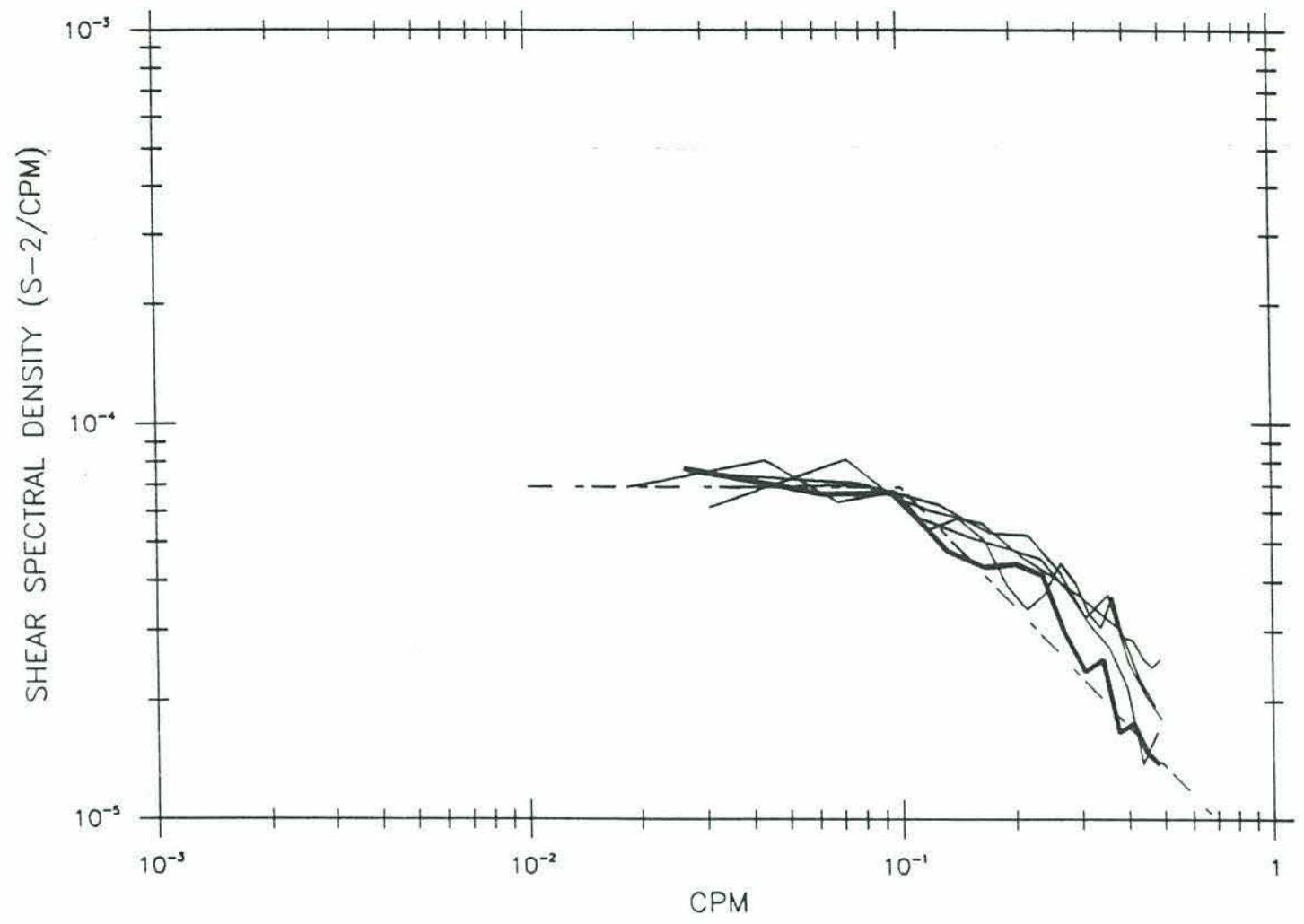

Figure 4.20: The TOPO_D2-D6 shear spectra, having been scaled in the vertical by $1 / \mathrm{EN}^{2}$ and the horizontal by $\mathrm{E}$, and then divided by two. The thick line represents data from the interval $200-400 \mathrm{~m}$, the four thin lines data from 400-1000, 1000-1500, 1500-2000 and $2000-3000 \mathrm{~m}$. The spectrum for the highest buoyancy frequency lies below the rest and potentially exhibits some effects of saturation. 


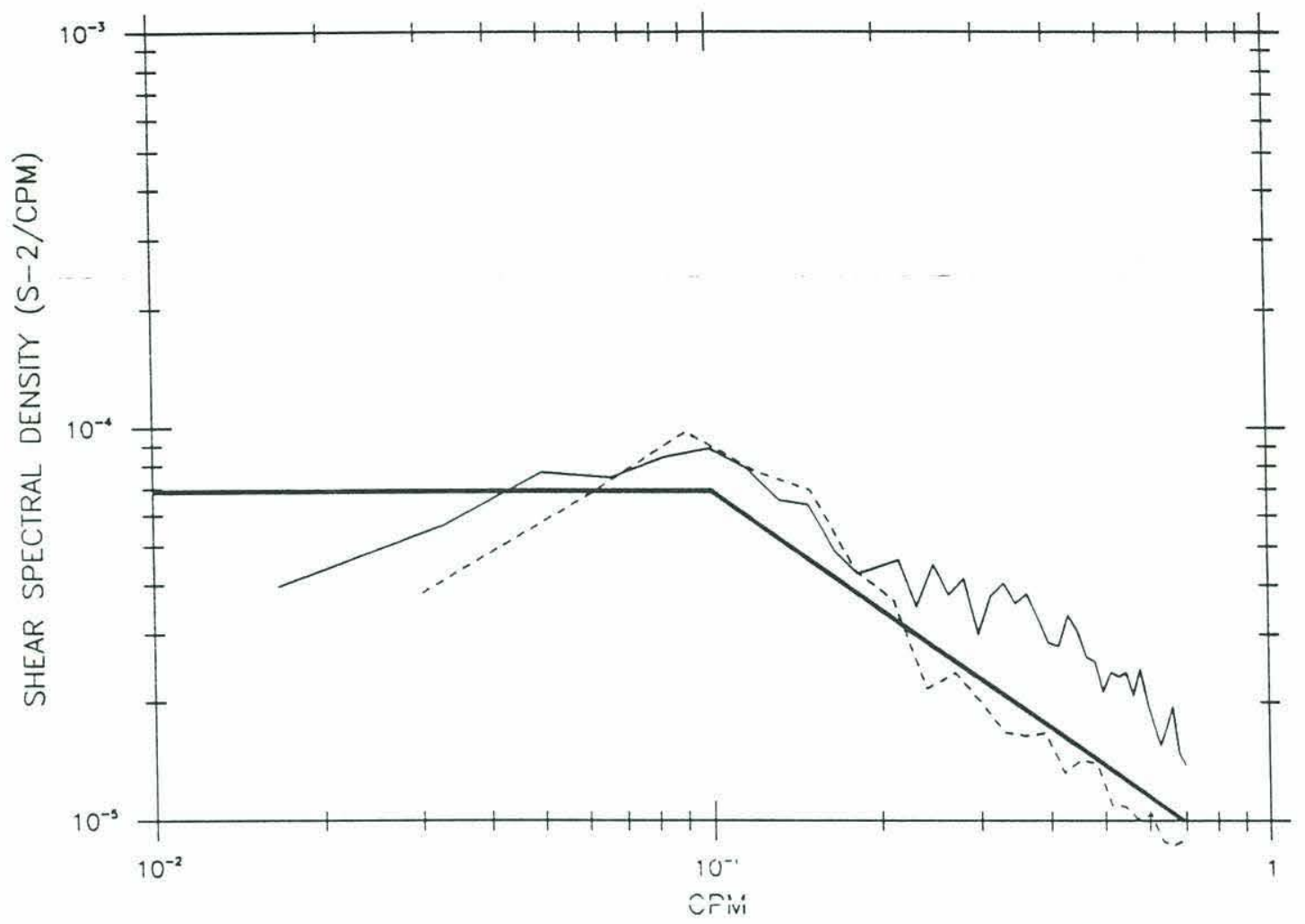

Figure 4.21: Shear spectra scaled in the vertical by $1 / \mathrm{EN}^{2}$ and in the horizontal by $1 / \mathrm{E}$, and then divided by 2 . The thin line represents the TOPO_F data and the dashed line the WRINCLE data. The high wavenumber slopes for the WRINCLE data are substantially smaller than that of the TOPO_F spectrum and the low buoyancy frequency spectra from the TOPO_DEEP data (Figure 4.21). 
frequency content) and TOPO_F (higher than average frequency content) shear spectra which also appears in Chapter Three (Figure 3.12). The slope of the WRINCLE shear spectra at high wavenumbers is much steeper than that of the TOPO_F spectra. This is consistent with the WRINCLE spectrum being saturated. Due to the peaked nature of the spectra about $\mathrm{m}_{\mathrm{c}}$ it is difficult to ascertain if there is any difference in the cutoff wavenumbers.

The third variable controlling the relative saturation of the shear spectrum is the Coriolis parameter. Lower values of $\mathrm{f}$ lead to a greater scale separation. Gregg et al. (1991) finds the shear spectra from data taken at 6 and $11 \mathrm{~N}$ to exhibit steeper than -1 slopes. Again, we find this to be consistent with the spectrum being influenced by saturation processes.

This study could be extended in three ways. First, it is possible to employ the Desaubies and Gregg N ${ }^{2}, \chi_{2}^{2}-S^{2}$ Richardson number pdf to produce an estimate for the shear spectral density as a function of wavenumber rather than employing the coarse power law specification as was done above. A second approach may be to numerically simulate a GM wavefield, mix those portions of the resulting Richardson number profile which are supercritical, and then compare the resulting spectra with the original to ascertain the length scales which are affected by the turbulent momentum fluxes. The most fruitful approach maybe simply to acquire more data to test the dependence of the internal wave spectral shapes upon $\mathrm{N}$, f and frequency content. 


\subsection{Conclusion and Discussion}

In Sections 4.2-4.4 the parameterization and scaling of the Richardson number based mixing model of KWB was examined. It was determined that the KWB model provides an effective parameterization of the rate of dissipation for the data in Chapter Three which exhibits a scaling of $\mathrm{E}^{2} \mathrm{~N}^{2}$. The nominal scaling of the model was determined to be $\mathrm{E}^{2} \mathrm{~N}^{3}$ on the basis of a number of theoretical Richardson number probability distributions. The nominal scaling of the Kunze et al. model is altered to $\mathrm{E}^{2} \mathrm{~N}^{2}$ due to the influence of a statistical dependence between $\mathrm{N}^{2}$ and $\mathrm{S}^{2}$ which reduces the observed occurrence of $\mathrm{Ri} \leq 1 / 4$ values. The statistical dependence was hypothesized to be an effect of the turbulent momentum and buoyancy fluxes on the internal wave shear and strain profiles which results in an increase of the Richardson number. The statistical dependence between $\mathrm{N}^{2}$ and $\mathrm{S}^{2}$ exhibits a buoyancy scaling which was interpreted as resulting from the decreasing ratio between the time scale of the shear instability mechanism and the adiabatic time scale of the internal wave field.

Application of the Kunze et al. parameterization necessitates the resolution of supercritical Richardson number events. Consistent estimates of the rate of dissipation of turbulent kinetic energy are possible if $\left\langle\mathrm{S}^{2}\right\rangle>1.0 \mathrm{~N}^{2}$ (Figure 4.5 ). The $\left\langle\mathrm{S}^{2}\right\rangle>1.0 \mathrm{~N}^{2}$ criterion may be an underestimate: consistent estimates also depend on resolving the $\mathrm{N}^{2}-\mathrm{S}^{2}$ correlation. If, as suggested in Section 4.5, this correlation is associated with the effects of the turbulent momentum flux upon the shear field, then the parameterization of $\varepsilon$ may require the 
resolution of length scales which participate in the momentum flux associated with a production/dissipation balance. This requirement may be sufficiently stringent to limit the applicability of the Kunze et al. parameterization. On the other hand, if the pertinent scales can be resolved, the Kunze et al. parameterization can be applied easily to internal wave fields which may exhibit nonequilibrium conditions with respect to the nonlinear wave/wave interaction mechanisms. No corrections need be made for deviations of the frequency or vertical wavenumber spectra from GM specifications, vertical anisotropy and horizontal inhomogeneity need not be assessed, and the presence of a background flow is inconsequential.

In Section 4.5 the saturated spectral theories and the Doppler shifting mechanism were considered in greater detail A method was developed for assessing the length scales which may be affected by the turbulent momentum fluxes associated with shear instability. It was determined that the maximum length scale is given by $\mathrm{mS}_{\mathrm{z}}=\mathrm{N}^{2} / 2$ where $\mathrm{S}_{\mathrm{z}}$ is the vertical wavenumber shear spectral density. For low stratifications and high internal wave frequency content this length scale is an overestimate and the shape of the spectrum will be controlled by the Doppler shift dominated internal wave/wave interactions. The controlling parameter is the ratio between the shear instability time scale, $O \sim(2 \pi / \mathrm{N})$ and the linear adiabatic time scale $\mathrm{O} \sim(\mathrm{Nf})^{-1 / 2}$ which is determined by the internal wave frequency content and the Coriolis parameter. If a scale separation does not exist between these two, supercritical Richardson number events will be terminated by wave propagation effects before they develop into a turbulent state and the energy contained 
in those scales will be fluxed up wavenumber adiabatically by the wave/wave interactions on a time scale $\mathrm{O} \sim \mathrm{f}^{-1}$. In such a case the turbulent momentum fluxes occur over smaller length scales where the time scale of the linear shear instability process is smaller due to the increase in rms shear. In the limit of small N/f (e. g. $4 \pi$ ), the growth rates may not be sufficiently large to establish shear instability as an intermediate mechanism linking the wave/wave interactions to turbulence. 


\section{Chapter Five}

\section{Conclusion}

This thesis has provided a background with which to be able to predict global patterns of mixing due to internal waves. This background results primarily from the parameterization studies in Chapters Three and Four. The parameterization studies are conducted in terms of a number of dynamical and kinematical models and a rather significant byproduct is a greatly improved knowledge of internal wave dynamics. Given the success of the parameterization studies, the problem of predicting global patterns of mixing due to internal waves is reduced to a problem of assessing the climatology of the internal wave field: defining the efficacy of various wave generation mechanisms and the ability of internal waves to interact with their environment to produce localized regions of heightened energy levels.

The results of the parameterization studies have immediate implications for oceanic mixing driven by internal wave motions. First, in agreement with the results of Gregg (1989), the background mixing rates for the GM spectral representation in Munk (1981) are small: $\mathrm{K}_{\rho}\left(\mathrm{E}_{\mathrm{GM}}\right)$ is approximately $.03 \times 10^{-4} \mathrm{~m}^{2} / \mathrm{s}$. Secondly, since the data were inconsistent with an inverse scaling of $K_{\rho}$ with $N$ at constant $\mathrm{E}$, some process or collection of processes must be responsible for heightened $\mathrm{E}$ values in the abyss if internal waves are responsible for producing the $\mathrm{O}\left(1-10 \times 10^{-4} \mathrm{~m}^{2} / \mathrm{s}\right)$ diffusivities generally inferred from deep ocean hydrographic data. We view 
internal wave reflection and/or internal wave generation associated with topographic features to be likely candidates. In Figure 5.1, $100 \mathrm{~m}$ averages of the diffusivity are plotted for the three high energy (high diffusivity) and five low energy (low diffusivity) profiles separately below $2000 \mathrm{db}$. The average diffusivity of the three TOPO Deep stations on the flank of Fieberling Guyot exhibits a trend to large values with depth. These profiles were terminated approximately $400 \mathrm{~m}$ from the bottom where the average diffusivity is presumably much larger than $1 \times 10^{-4} \mathrm{~m}^{2} / \mathrm{s}$. Construing a global average on the basis of three profiles is a tenuous thing: more data needs to be collected and the processes which contribute to the heightened internal wave energy levels quantified. It is too soon to say if the heightened energy levels associated with topographic features can result in an averaged diffusivity equal to the $1 \times 10^{-4}$ $\mathrm{m}^{2} / \mathrm{s}$ of Wyrtki's (1961) analysis. Deep western boundary currents present potential critical layer for both freely (downward) propagating and upward propagating, topographically generated lee waves. The association of heightened internal wave energy levels in regions of strong mean flow (Chapter Two) raises the question of whether significant internal wave/mean flow interaction is associated with deep western boundary currents. Defining the role of internal wave driven mixing would be greatly furthered by a coordinated experiment making use of both dissipation measurements and hydrographic data to define the advective and diffusive heat fluxes in a closed basin.

Globally heightened internal wave energy levels associated with topographic features would presumably lead to an increased 


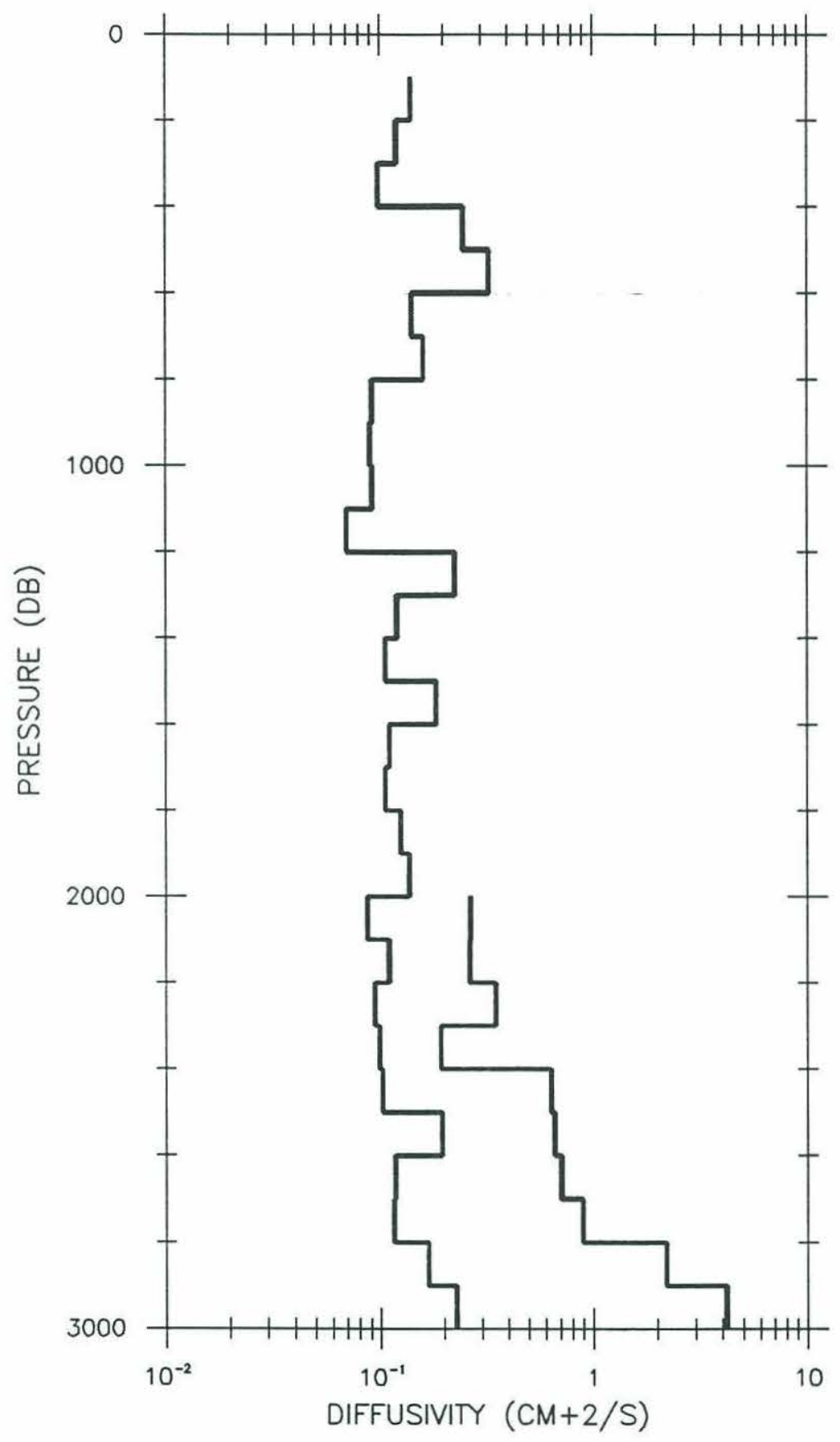

Figure 5.1: One hundred $\mathrm{db}$ averages of $\mathrm{K}_{\rho}$ for the TOPO_Deep data set. Below $2000 \mathrm{db}$ a subset of the data exhibited heightened diffusivities and internal wave energy levels; that subset has been treated separately. The profiles exhibiting heightened turbulence extended to within approximately 400 meters of the bottom. The other profiles were terminated more than $1000 \mathrm{~m}$ off the bottom. 
diapycnal buoyancy flux at the boundary. Such boundary layers generate interior flows in a manner analogous to Ekman layer transports (see Garrett, 1990 for a review). The dynamical implications of this scenario for the general circulation are not obvious, however. The theoretical problems associated with boundary mixing deserve a great deal more attention. An understanding of the dynamics of processes at small vertical scales and short time scales may be necessary to understand the dynamics of motions on a basin scale and the large scale thermodynamic structure of the oceans.

An internally consistent picture of mixing due to internal waves results from this study. Wave/wave interactions on scales of tens to hundreds of meters transport energy through the vertical wavenumber spectrum to smaller scales. The magnitude of the average upwavenumber flux and its functional dependence upon the internal wave energy level, background stratification and frequency content is best described by the model of Henyey, Wright and Flatte (1986) (Chapter Three). This upwavenumber transport leads to the accumulation of variance at scales smaller than 10 meters. The accumulation of variance at these scales leads in turn to the occurrence of shear instability in regions where the Richardson number is locally supercritical (i.e. $\mathrm{Ri}<1 / 4$ ). The presence of supercritical Richardson number permits the growth of small-scale waves which break and result in turbulence. The effect of the turbulence is to redistribute the momentum and buoyancy in the background over somewhat larger scales to produce a background flow field with higher Richardson number. The Richardson number 
scaling model of Kunze, Williams and Briscoe (1990) produced estimates for the rate of dissipation of turbulent kinetic energy consistent with the estimates from the nonlinear wave/wave interaction model of Henyey et al. (Chapter Four).

The nonlinear wave/wave interaction theories (Henyey et al.; McComas and Muller, 1981) equate the rate of turbulent dissipation with the average energy flux through the vertical wavenumber spectrum. This is true at any vertical wavenumber as long as the model spectrum which forms the basis for the analysis is statistically stationary with respect to the nonlinear interactions. Otherwise the role of the wave/wave interactions will partially be to redistribute the energy in wavenumber/frequency space without resulting in turbulent dissipation in the high vertical wavenumber portion of the spectrum. Such effects may be associated with the presence of a background flow, vertical anisotropy, horizontal inhomogeneity and deviations in the frequency and wavenumber spectra from the GM model. In such cases it is not clear that a simple parameterization in terms of the average shear spectral density over scales of tens to hundreds of meters and the background stratification will provide an adequate parameterization of the turbulent dissipation.

In Chapter Three a data set in the far field of a seamount in a region of negligible larger scale flow was examined to address the issue of buoyancy scaling of the turbulent dissipation rate. These data had little variation in energy level or frequency content. No significant differences were found between the $\mathrm{CW}$ and $\mathrm{CCW}$, the North/South and East/West or along- and across-slope shear spectra. Thus these data were consistent with vertical isotropy and horizontal 
homogeneity. The vertical wavenumber shear spectra for these data were collapsed under the canonical scaling (e.g. Gargett, 1990;

Appendix Two). The observed spectra were thus consistent with the GM spectrum (e.g. Munk, 1981) except for the presence of energy levels a factor of 2-3 above the GM level and frequency content a factor of two lower. This is roughly consistent with the factor of two universality quoted for the GM spectrum (Munk, 1981). The observed diffusivities and the model prediction of Henyey et al. were found to be consistent.

Data from a warm core ring and from a region of steep topography were also analyzed in Chapter Three in order to examine how deviations from the assumed GM spectrum may effect the observed dissipation rates. A background flow was obviously present in the region from which the warm core ring profiles were obtained. In addition these data were noted to be vertically anisotropic, to be dominated by near-inertial frequencies and to have a rather peaked vertical wavenumber spectrum in comparison to the canonical shear spectrum. The data from a region of steep topography were also noted to have a peaked vertical wavenumber spectrum and were characterized by higher frequency motions. The only obvious trend in the diffusivities vs the model predictions based upon energy levels and buoyancy frequency was in terms of the frequency content. It was then argued that for small perturbations in frequency content the scaling of the average energy fluxes at high vertical wavenumber for a spectrum which was not in equilibrium with respect to wave/wave interactions retained the scaling of individual waves propagating in an otherwise statistically stationary 
(nominally GM) wavefield. For the frequency scaling of the Henyey et al. model, this approach resulted in reducing more than an order of magnitude scatter in the diffusivity estimates scaled by only energy level and background stratification to a scatter of less than a factor of four. The frequency content was estimated from the shear strain ratio. This method is equivalent to assuming the internal wave field consists of a single frequency. A better estimate of the frequency dependence of the high wavenumber energy flux might be the spectrally (frequency domain) weighted value of $\left(\omega^{2}-f^{2}\right)^{1 / 2}$. However, such information is not available from single vertical profiles. In Chapter Two evidence was provided which implies the internal wave field in an upper ocean front was interacting with and modified by the background flow. Horizontal inhomogeneity and vertical anisotropy were noted in that data set. Despite the presence of these apparent non-equilibrium conditions, the model of Gregg (1989), which in turn was based upon the model of Henyey et al., effectively collapsed the observed diffusivity estimates. The frequency content of that data set could not be addressed in a manner similar to Chapter Three due to the presence of a thermostad within the front. Nevertheless, these results imply that the presence of a background flow, vertical anisotropy, horizontal inhomogeneity and deviations from the canonical shear spectrum are not particularly important in determining the average energy flux through the vertical wavenumber spectrum at high wavenumbers. The primary variability appeared to be associated with the frequency content. These results could, and should, be verified at a theoretical level. 
In sections 4.2-4.4 the parameterization and scaling of the Richardson number based mixing model of Kunze et al. (1990) was examined. It was determined that the Kunze et al. model provides an effective parameterization of the rate of turbulent dissipation for the data examined in Chapter Three which exhibits a scaling of $\mathrm{E}^{2} \mathrm{~N}^{2}$. The nominal scaling of the model was determined to be $E^{2} \mathrm{~N}^{3}$ on the basis of a number of theoretical Richardson number probability distributions. This nominal scaling of the Kunze et al. model is altered to $\mathrm{E}^{2} \mathrm{~N}^{2}$ due to the influence of a statistical dependence between $\mathrm{N}^{2}$ and $\mathrm{S}^{2}$ which reduces the observed occurrence of $\mathrm{Ri} \leq 1 / 4$ values. The statistical dependence was hypothesized to be an effect of the turbulent momentum and buoyancy fluxes on the internal wave shear and strain profiles which results in an increase of the Richardson number. The statistical dependence between $\mathrm{N}^{2}$ and $\mathrm{S}^{2}$ exhibited a buoyancy scaling which was interpreted as resulting from the decreasing ratio between the time scale of the shear instability mechanism and the adiabatic time scale of the internal wave field.

In section 4.5 the saturated spectral theories and the Doppler shifting mechanism were considered in greater detail A method was developed for assessing the length scales which may be affected by the turbulent momentum fluxes associated with shear instability. The maximum length scale is given by

$$
\int_{0}^{\mathrm{m}} \mathrm{S}_{\mathrm{z}} \mathrm{dm}=\mathrm{N}^{2} / 2 \text {. }
$$


For low stratifications and high internal wave frequency content this length scale is an overestimate and the shape of the spectrum will be controlled by the Doppler shift dominated internal wave/wave interactions. The controlling parameter is the ratio between the shear instability time scale, $\mathrm{O} \sim(2 \pi / \mathrm{N})$ and the linear adiabatic time scale $\mathrm{O} \sim(\mathrm{Nf})^{-1 / 2}$ which is determined by the internal wave frequency content and the Coriolis parameter. If a scale separation does not exist between these two, supercritical Richardson number events will be terminated by linear wave propagation effects before they develop into a turbulent state and the energy contained in those scales will be fluxed up wavenumber adiabatically by the wave/wave interactions on a time scale $\mathrm{O} \sim \mathrm{f}^{-1}$. In such cases the turbulent momentum fluxes occur over smaller length scales where the time scale of the linear shear instability process is smaller due to the increase in rms shear. In the limit of very small $\mathrm{N} / \mathrm{f}$ (e.g. $\mathrm{f}^{-1} \sim 2$ buoyancy periods), the growth rates may not be sufficiently large to establish shear instability as an intermediate mechanism linking the wave/wave interactions to turbulence.

While we view the results of the study to be robust, the analysis would benefit from the use of a single data set with a large number of degrees of freedom, dissipation data, a fully resolved internal wave shear spectrum, and an order of magnitude variation in the background stratification. The arguments forwarded concerning the relative control of the shape of the shear spectrum by wave/wave interaction vs shear instability in the transition region could be verified through the acquisition of more data to examine the dependence on $\mathrm{N}, \mathrm{f}$ and frequency content. The crude methodology 
for assessing the length scales affected by the turbulent fluxes could be refined and a number of suggestions for that were made in Chapter Four.

Application of the Kunze et al. parameterization necessitates the resolution of supercritical events. Consistent estimates of the dissipation rate are possible if $\left\langle\mathrm{S}^{2}\right\rangle>1.0 \mathrm{~N}^{2}$. This criterion may be an underestimate: consistent estimates also depend on resolving the $\mathrm{S}^{2} / \mathrm{N}^{2}$ correlation. If this correlation is associated with the effects of the turbulent momentum fluxes upon the shear field, then the parameterization of $\varepsilon$ may require the resolution of the length scales which participate in the momentum flux associated with a production/dissipation balance. This length scale requirement may be sufficiently small as to limit the applicability of the KWB parameterization. On the other hand, if the pertinent scales can be resolved, the KWB parameterization can be applied easily to internal wave fields which exhibit non-equilibrium conditions with respect to the nonlinear wave/wave interactions. No corrections need to be made for deviations of the frequency or wavenumber spectra from the GM prescription, vertical anisotropy and horizontal inhomogeneity need not be assessed, and the presence of a geostrophic background flow is inconsequential.

The velocity and density data examined herein were collected using vertical profiling instrumentation specifically designed to resolve velocity and density differences associated with the internal wave field over vertical scales of $\mathrm{O} \sim 1 \mathrm{~m}$. Other profiling instrumentation with coarser resolution may be used in conjunction with the dynamically based parameterizations discussed in Chapter Three. 
Expendable Current Profilers (XCPs) resolve vertical scales of $10 \mathrm{~m}$ at thermocline stratification rates. The analysis of Gregg (1989) is based in part upon XCP measurements. A lower stratifications, the $\mathrm{XCP}$ noise spectrum begins to swamp the internal wave signal at small vertical scales (e.g. Gregg and Kunze, 1991). The XCP only measures temperature and thus utilization of the frequency based correction may only be possible in regions exhibiting a tight $\theta-\mathrm{S}$ relationship. The XCP has a maximum depth range of $1600 \mathrm{~m}$ and is therefore limited in its capacity to measure the deep ocean internal wave field.

It may prove possible to apply the parameterization schemes discussed in Chapter Three to current meter data. Since the energy level (E) parameterization is a function of the small-scale shear rather than the velocity which is dominated by the largest scales, this application would require closely spaced (on the order of 10-100 meters) current meters. Unfortunately, such closely spaced sampling schemes are not routinely encountered in the historical current meter data base.

It should ultimately prove possible to utilize the dynamical parameterizations in numerical modeling schemes. The parameterization studies result in a prediction for the diffusivity as a function of the frequency and vertical wavenumber content of the internal wave field. Given the internal wave information, it should be possible to determine the magnitude and vertical decay scale (Figure 5.1) of a diffusive boundary layer associated with internal wave driven mixing for a given internal wave field. Determination of the internal wave field requires defining the characteristics 
associated with the various generation/interaction mechanisms (e.g. Eriksen, 1985). Utilization of this information in a numerical model may be possible in terms of environmental parameters such as topographic slope, average barotropic tidal velocities and background flow speeds.

The dynamical models of nonlinear wave/wave interactions examine only the mid-latitude case. While a great deal of work has been done in the upper ocean at the equator in the presence of the equatorial undercurrent, we are not aware of any published concurrent internal wave and dissipation measurements below the undercurrent. The reports of $10-30 \% \mathrm{Ri}<1 / 4$ over $5 \mathrm{~m}$ scales by Toole and Hayes (1984) in the Eastern Equatorial Pacific (250-900db) implies that internal wave driven mixing may be quite large beneath the undercurrent. Expanding to the equatorial regime may prove very fruitful on both an observational and theoretical level.

We now know how to parameterize the mixing due to internal waves. In magnitude and scaling, the observed diffusivities are remarkably consistent with the dynamical model of Henyey et al. If internal waves are responsible for the heightened diffusivities generally estimated for the abyss, then such diffusivities must be associated with heightened internal wave energy levels. The processes which may potentially give rise to heightened energy levels need to be examined on both observational and theoretical grounds. Such heightened internal wave energy levels presumably give rise to a heightened diapycnal buoyancy flux at the boundary. The dynamics and implications of boundary mixing (Garrett, 1990) need to be pursued. 


\section{Appendix One}

\section{Fine- and Microstructure Processing}

\section{A. Overview of the HRP}

The data in this study have been obtained primarily with the High Resolution Profiler (HRP), a free falling, internally recording profiler. Relative velocities are measured with an acoustic velocimeter. Profiles of oceanic velocity are computed from the relative velocity, accelerometer and magnitometer data using a variation of the TOPS model (Hayes et al., 1984). The HRP is a compound pendulum with a resonance frequency proportional to the fall rate divided by the profiler length. Relative flow about the profiler induces translation and oscillation. The relative velocity sensed with the acoustic current meter contains a component associated with both motions. At vertical scales equal to the profiler length, the accelerometer data is dominated by oscillatory motions. For FASINEX and TOPO, a nodding correction was applied to the current meter data using the accelerometers mounted at each endcap (see Schmitt et al., 1988 for details). For WRINCLE, the damage of one current meter axis dictated a different scheme to compute oceanic velocity profiles. For these data, the missing relative velocity data were estimated from the accelerometer data for vertical scales larger than the profiler length. Oceanic velocity profiles were then computed with the Hayes et al. (1984) "point-mass" model with no correction for nodding effects (J. Toole, personal communication). 
Conductivity, temperature and pressure are sensed with an NBIS Mark III CTD. Calibrations for these sensors were determined by laboratory measurements prior to each cruise. Conductivity scaling was subject to adjustment to give agreeement so that deep potential temperature / salinity curves were consistent with historical hydrographic data from each work area.

Microstructure quantities (two axes of shear, temperature and conductivity gradients) are sensed with airfoil probes (Osborn,1974; Osborn and Crawford 1980), an FP07 fast response thermistor (Gregg et al., 1986), and a dual electrode conductivity cell (Meagher et al., 1982). Both the temperature and conductivity microstructure sensor assemblies were purchased from Sea-Bird Electronics, Inc. Gradient variances were calculated after spectrally transforming, then applying spectral corrections for electronic and sensor transfer functions. The variances were determined by integrating the spectrum out to a relative minimum in the spectra to enhance the signal to noise ratios. Thus noise levels were a function of signal level. The microstructure processing algorithms are based upon those developed by N. Oakey (personal communication).

The profiler falls at a nominal rate of $.65 \mathrm{~m} / \mathrm{s}$. Finescale data is sampled at $10 \mathrm{~Hz}$, processed and then bin-averaged at $1 / 2 \mathrm{db}$ intervals. The microstructure data is sampled at $200 \mathrm{~Hz}$. The spectra were created from 256 points centered on each $1 / 2 \mathrm{db}$ bin, providing essentially independent estimates of $\varepsilon$ and $\chi$ every $1 / 2 \mathrm{db}$. 


\section{B. Microstructure}

The Cox-Osborn relation (Osborn and Cox, 1972) and Osborn's (1980) method interpret $\varepsilon$ and $\chi$ in terms of stationary, homogeneous, isotropic turbulence resulting from productiondissipation balances, with 'background' gradients only in the vertical:

$$
\begin{array}{ll}
\left.<u^{\prime} w^{\prime}><\frac{\mathrm{du}}{\mathrm{dz}}\right\rangle=-\frac{\mathrm{g}}{\rho}\left\langle w^{\prime} \rho^{\prime}\right\rangle-\varepsilon & \text { (Turbulent Kinetic Energy Equation) } \\
2<w^{\prime} T^{\prime}><\frac{\mathrm{dT}}{\mathrm{dz}}+\Gamma>=-\chi & \text { (Turbulent Heat Equation), }
\end{array}
$$

where $\varepsilon$ is the rate of dissipation of turbulent kinetic energy, $\chi$ is the rate of dissipation of thermal variance and $\Gamma$ is the adiabatic lapse rate. The flux Richardson number, $\mathrm{R}_{\mathrm{f}}$, is defined as the ratio between the buoyancy and momentum fluxes:

$$
\left.\mathrm{R}_{\mathrm{f}}=-\frac{\mathrm{g}}{\rho}<w^{\prime} \rho^{\prime}\right\rangle\left|<\mathrm{u}^{\prime} w^{\prime}\right\rangle<\frac{\mathrm{du}}{\mathrm{dz}}>\text {. }
$$

The flux Richardson number is generally assumed to be constant within the oceanographic context (see Rohr et al. (1984) for an insightful discussion). Values of $\mathrm{R}_{\mathrm{f}}=.15$ (Osborn, 1980) or $\mathrm{R}_{\mathrm{f}}=.2$ (Oakey, 1982) are generally assumed. Here we follow Oakey (1982) in employing $R_{f}=.20$. Given a constant flux Richardson number, the turbulent kinetic energy equation can be written as (Osborn, 1980):

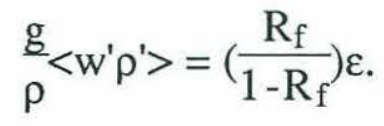

Diffusivities result from parameterizing the buoyancy flux $\left(\mathrm{K}_{\rho}\right)$ and the temperature flux $\left(\mathrm{K}_{\mathrm{T}}\right)$ as a constant times a gradient, i.e. the usual eddy coefficient formulation: 


$$
\begin{aligned}
& \mathrm{K}_{\mathrm{\rho}}=\frac{\mathrm{R}_{\mathrm{f}}}{1-\mathrm{R}_{\mathrm{f}}} \frac{\varepsilon}{\mathrm{N}^{2}}, \\
& \mathrm{~K}_{\mathrm{T}}=\frac{\chi}{2<\theta_{\mathrm{Z}}>^{2}} .
\end{aligned}
$$

Estimates of the rate of dissipation of turbulent kinetic energy, $\varepsilon$, are created by averaging the two components of shear variance and assuming isotropy (Yamazaki and Osborn, 1990). Estimates of the rate of dissipation of thermal variance, $\chi$, are produced also assuming isotropy.

$$
\begin{aligned}
& \varepsilon=7.5 \vee<\mathrm{u}_{\mathrm{z}}^{2}> \\
& \chi=6 \kappa<\mathrm{T}_{\mathrm{z}}^{2}>
\end{aligned}
$$

Given this approach, the problem of estimating the eddy diffusivity is reduced to one of estimating the turbulent temperature and velocity gradients as accurately as possible. Note also that an eddy diffusivity is not the sole interpretation for the microstructure measurements. The rate of dissipation of turbulent kinetic energy, $\varepsilon$, can be equated with the average flux of internal wave energy through the vertical wavenumber spectrum (e.g. McComas and Muller, 1981; Henyey et al., 1986).

The Reynold's decomposition which leads to the turbulent heat and kinetic energy equations implies a separation between mean and turbulent quantities. Gargett and Holloway (1984) point out that there is no scale separation between internal waves and turbulence. Given that the contribution of internal wave strain to potential temperature and buoyancy gradients is $\mathrm{O} \sim 1$ relative to the background gradients (Appendix Two), varying the length scale of 
the fit to the 'background' results in varying contributions due to internal wave straining and the mean. One approach to defining a mean buoyancy or potential temperature gradient would be to spatially average a number of temperature and salinity profiles. This approach will incur serious errors if there is large lateral variability in the background buoyancy and potential temperature gradients. This potential is realized in the interior of the FASINEX front and within the salinity compensated temperature inversions noted for the TOPO data (Chapter Three). Hence the background gradients were defined on the basis of individual profiles. From a pragmatic standpoint, the diffusivity estimates were found to be stable if the length scale used to estimate the background gradients was significantly larger than the Osmidov length. A length scale of approximately five $E_{1}$ meters was employed. The nominal length scale was $20 \mathrm{~m}$ between 0 to $2000 \mathrm{db}$ and $50 \mathrm{~m}$ from 2000 to 3000 $\mathrm{db}$ for the TOPO_DEEP data. This variability in nominal length scales was dictated by the presence of highly energetic regions next to the seamount. The nominal fit for the TOPO_F and WRINCLE data was $20 \mathrm{~m}$, and that for FASINEX $10 \mathrm{~m}$.

Additionally, it was required that $\mathrm{N}^{2}$ be greater than the square of the buoyancy frequency corresponding to four times the least bit in temperature. If this condition was not met the length scale of the least squares estimate of $\mathrm{N}^{2}$ was doubled. The minimum background potential temperature gradients were required to be greater than four least bits. Due to the presence of salinity compensated temperature inversions in the TOPO data sets, the 
background temperature gradients in the Cox-Osborn model were calculated as least square fits to $|\Delta \mathrm{T} / \Delta \mathrm{z}| \Delta \mathrm{z}$ above $2000 \mathrm{~m}$ for that data.

The microstructure data was electronically premphasized prior to digitization to reduce quantization errors. The temperature and conductivity transfer functions are white at low frequencies, resemble first-difference operators (i.e. $\omega^{2}$ ) between .1 and $200 \mathrm{~Hz}$, and are white at higher frequencies. The data from the air-foil velocity probes are premphasized with a filter which resembles a first-difference operator until $100 \mathrm{~Hz}$, where upon it falls as $\omega^{-2}$. Four-pole Butterworth filters with half-power points at $100 \mathrm{~Hz}$ are additionally applied to all microstructure channels as anti-aliasing filters.

The first step in the microstructure processing is to calibrate the micro-sensor data. Laboratory calibrations provided by N. Oakey (BIO) for the FASINEX and TOPO data and R. Lueck (Univ. of Victoria) (WRINCLE) were applied to the shear probes. The microtemperature and microconductivity data were calibrated with local regressions against the insitu CTD data. In order to accomplish this, the effects of the premphasis filter were removed with a recurrsive digital filter described in greater detail in Schmitt et al., 1988. The microtemperature and microconductivity data were despiked with a first difference operator, corrected for the electronic premphasis with a recurrsive filter (Schmitt et al., 1988), and then averaged over $1 / 2 \mathrm{db}$ intervals. The data were then regressed to the $1 / 2 \mathrm{db}$ CTD data in fits of 21 points. A two-hundred point running mean of the microtemperature and microconductivity calibrations was then taken, and points that deviated from the running mean by greater 
than $10 \%$ were replaced by the mean. The calibrations were then smoothed twice with a 51 point box-car filter. Vertical profiles of microtemperature and microconductivity calibrations are repeatable to within $\pm 5 \%$.

The premphasized microstructure data was analyzed as a series of 256 points centered on the $1 / 2 \mathrm{db}$ spaced finestructure data. The microstructure data was read in and a two-pole, $10 \mathrm{~Hz}$ Butterworth recurrsive filter applied to the shear probe data to reduce the amplitude of a vibrational spike at $50 \mathrm{~Hz}$ (Figure A.1.1 a,b). The calibrations were then applied to the individual data points. After calculating the fall rate, the data was transformed from a time series to a spatial series by invoking Taylor's hypothesis. The next step in processing was to check for the presence of spikes. The 256 points were divided into 10 separate intervals and variances calculated for each of those intervals. The variances were ordered in magnitude and the smallest nine summed. If the largest subinterval variance exceeded 3-6 times the sum of the nine others, that variable and segment of 256 points was flagged as being potentially noisy. A similar scheme was discussed by Marmarino et al., 1986. Such spikes are due to a number of causes: a $10 \mathrm{kHz}$ transponder used for tracking the profiler, plankton impacts and fouling of the microconductivity/temperature sensors by detritus.

The 256 points were then detrended with a least squares fit, windowed with $50 \%$ cosine tapers (Hanning window) and then fastFourier transformed. The microconductivity/microtemperature cospectrum was also computed. Spectral density estimates were created by multiplying the Fourier coefficients by their complex 
conjugates and corrected for the loss of variance implied by the windowing procedure.

The spectral corrections were then applied to the spectral density estimates. The corrections come in three basic flavors. The first is for the recurrsive filter applied to the shear probe data. The spectral characteristics of the applied filter can be computed exactly and the correction is straightforward. Secondly, the theoretical and measured electronic premphasis and anti-aliasing filters agree to within $\pm 5 \%$ and thus can be easily accounted for. The third variety of spectral corrections represent the transfer functions of the microsensors.

The airfoil velocity probe spatially averages the three dimensional turbulent velocity field in a complicated manner which depends on the turbulent energy spectrum (Ninnis, 1984). In FASINEX shear probes designed by Dr. N. Oakey (Bedford Institute of Oceanography) were used. A single-pole transfer function with a half-power point at $2 \mathrm{~cm}$ was used to correct the shear spectra. This approach was taken so that the HRP and EPSONDE data would be consistent in this respect (Oakey, 1977). In later experiments, probes constructed by Mr. Edmond Schiemer to a design of Dr. R. Lueck were employed. The algebraic correction of Ninnis (1984) was used to correct these later shear spectra.

The response time of the thermistor is limited by the time for temperature fluctuations to diffuse through the glass coating and fluid boundary layer (which is related to the rate of descent) surrounding the sensor. The spectral response of microbead thermistors has been well documented (Lueck et al., 1977; Gregg et 
al., 1978; Lueck and Osborn, 1980; Gregg and Meagher, 1980). Lueck et al. (1977) originally suggested that the transfer function should be aptly described by a single-pole transfer function for small frequencies and that no asymptotic high frequency functional form was apparent from their analytical results. Gregg and Meagher (1980), in a later work, found that the high frequency dynamic response of such thermistors was sufficiently well described by a double-pole transfer function. In practice, for fall speeds of $.6 \mathrm{~m} / \mathrm{s}$ there appeared to be little difference in Gregg and Meagher's fitted single and double-pole transfer functions as long as the gain correction did not exceed an order of magnitude.

The HRP FP07 thermistor data was corrected assuming as a single-pole transfer function. The time constant was determined from fits to the ratio between the microconductivity and microtemperature spectra for regions of large density ratio and large $\chi$. The criterion of large density ratio ensures that the salinity spectrum has a small contribution to the conductivity. The criterion of large $\chi$ ensures the transfer function will not be affected by either sensor's noise spectrum. The analysis was repeated for each individual thermistor used. The single-pole form was employed as it appeared to give a better representation of the transfer function at low frequencies for the HRP data.

The microconductivity cell was not corrected for any effects of spatial averaging. The dual needle cell had a centerline to centerline separation of $3 \mathrm{~mm}$. The electrical field of such a sensor is approximately a dipole with $95 \%$ of the field within two centerline separations (Head, 1983). Given a sampling rate of $200 \mathrm{~Hz}$ and a fall 
speed of $.65 \mathrm{~m} / \mathrm{s}$, the Nyquist wavenumber, $1 / 6 \mathrm{~mm}$, is roughly equal to the averaging interval. Meagher et al. (1982), Okawa and Dugan (1984) and Dugan and Stalcup (1988), have established that the halfpower point of the conductivity probe is approximately $100 \mathrm{cpm}$. Frequencies of less than $50 \mathrm{~Hz}$ were therefore relatively unaffected by any effects of averaging by the needle cell.

After the spectra were corrected for the various transfer functions, the gradient variances were then calculated. A nine point box-car filter was applied to the spectral density estimates for individual $1 / 2 \mathrm{db}$ spectra and a spectral minimum defined on the basis of the averaged spectra. The minimum was defined as the first relative minimum encountered as one progresses from high frequencies to low. For the microtemperature and microconductivity the upper and lower frequencies for this calculation were 80 and 20 $\mathrm{Hz}$, respectively. For the shear probe data the upper and lower bounds were 40 and $6.25 \mathrm{~Hz}$, respectively. The gradient variances were then calculated by integrating the unaveraged spectra out to the spectral minimum. If the vertical shear variance for one component was determined to be less than a corresponding dissipation rate of $5 \times 10^{-10} \mathrm{~W} / \mathrm{kg}$, the upper bound for the spectral minimum calculation was lowered to $12.5 \mathrm{~Hz}$. This is done to eliminate a secondary noise peak in the shear spectra (see Figure A.1.1 a,b).

After calculating the variances, the ratio between shear components and microtemperature and conductivity was computed. If the ratio between shear components exceeded three and the ratio between temperature and conductivity exceeded six, the spectra 
were flagged as being potentially noisy. A segment was also flagged if the variance of any variable exceeded a specified magnitude. The chosen magnitudes usually represent the largest several percent of the variances for a statistically homogeneous portion of the data set. The spectra for segments which were flagged were then graphically displayed. A spectrum was regarded as noisy if it failed to reveal a spectral minimum. A flagged spectrum was also regarded as noisy if substantial agreement between redundant sensors, i.e. the two shear probes and the microconductivity-microtemperature sensors did not exist. Spectra which were regarded as noisy were not included in the latter part of the analysis.

The dissipation noise levels were approximately $1 \times 10^{-11} \mathrm{~W} / \mathrm{kg}$, roughly a decade smaller than the lowest published noise levels for $\varepsilon$ (Gregg and Sanford, 1988; Oakey, 1988). Noise levels for $\chi$ were likewise small, roughly $2 \times 10^{-12}{ }^{\circ} \mathrm{C}^{2} / \mathrm{m}$. Figures A.1.1 a-d represent data between 2500-2985 $\mathrm{db}$ from the southern-most profile in the TOPO_DEEP data set (Figure 3.4). This profile had the lowest bin averaged estimates of $\varepsilon$ and $\chi\left(4.1 \times 10^{-11} \mathrm{~W} / \mathrm{kg}\right.$ and $\left.4.7 \times 10^{-12}\right){ }^{\circ} \mathrm{C}^{2} / \mathrm{s}$, respectively.) Figures A.1.1 a,b consist of 970 different shear spectra for one probe which have been averaged into bins according to the relative size of the dissipation estimates. The large peak at high frequency is believed to be due to vibration. The small peak at low frequencies is of unknown origin. Twenty individual shear spectra do not appear in the averages as they were edited due to the fact that they did not exhibit a clear spectral minimum. Based upon this criterion, $2-4 \%$ of the data is routinely edited. Individual spectra were averaged into bins of $1 \times 10^{-12}-2 \times 10^{-12}, 2 \times 10^{-12}-5 \times 10^{-12}$, 


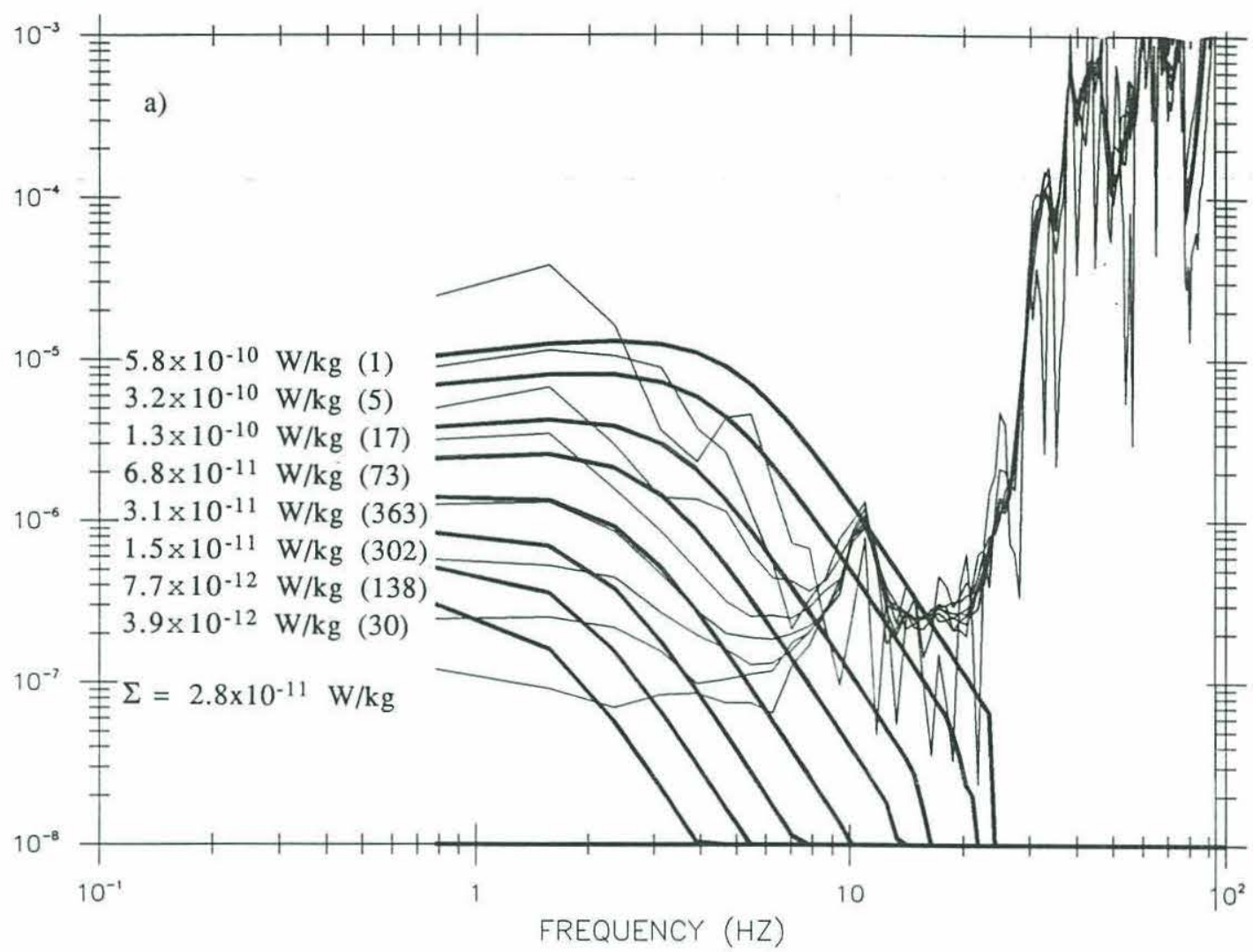

Figure A.1.1: Bin averaged estimates of the microstructure spectral density estimates (thin lines) for profile 74 from the TOPO experiment, $2500 \leq \mathrm{p} \leq 2985 \mathrm{db}$. The universal and Batchelor spectra (Oakey, 1982) appear as the thick curves. a) x-component of shear b) y-component of shear c) temperature gradient d) conductivity gradient 


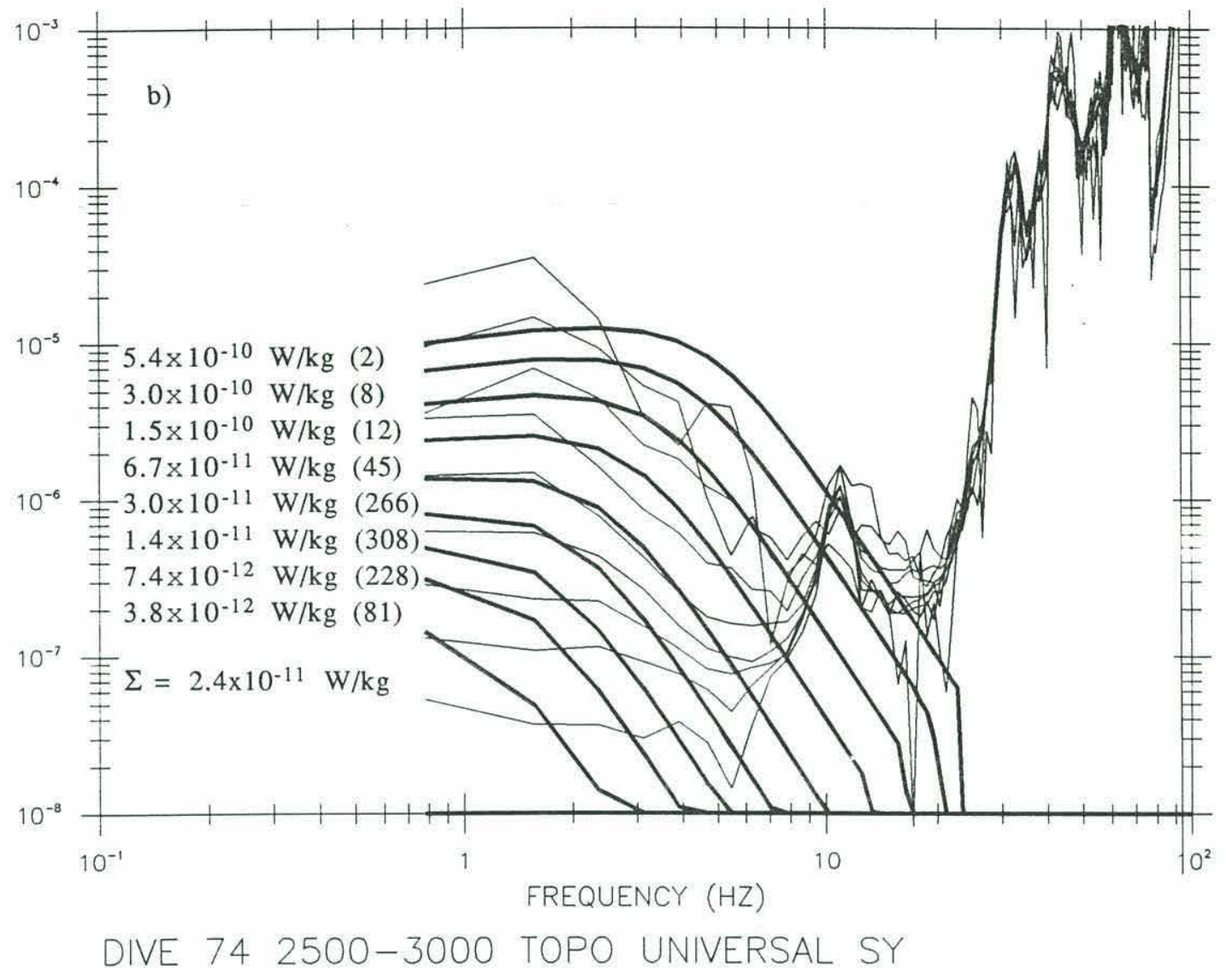




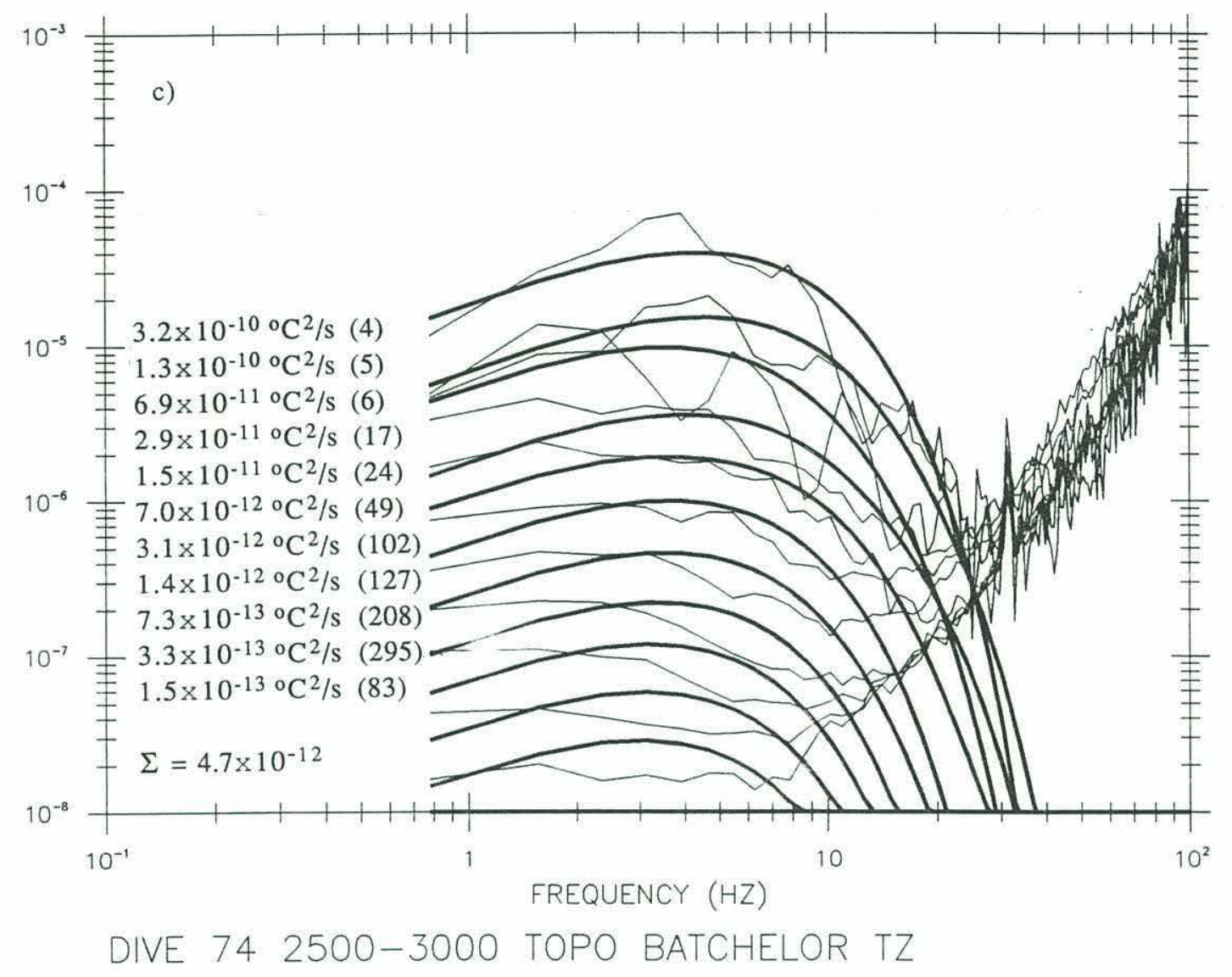




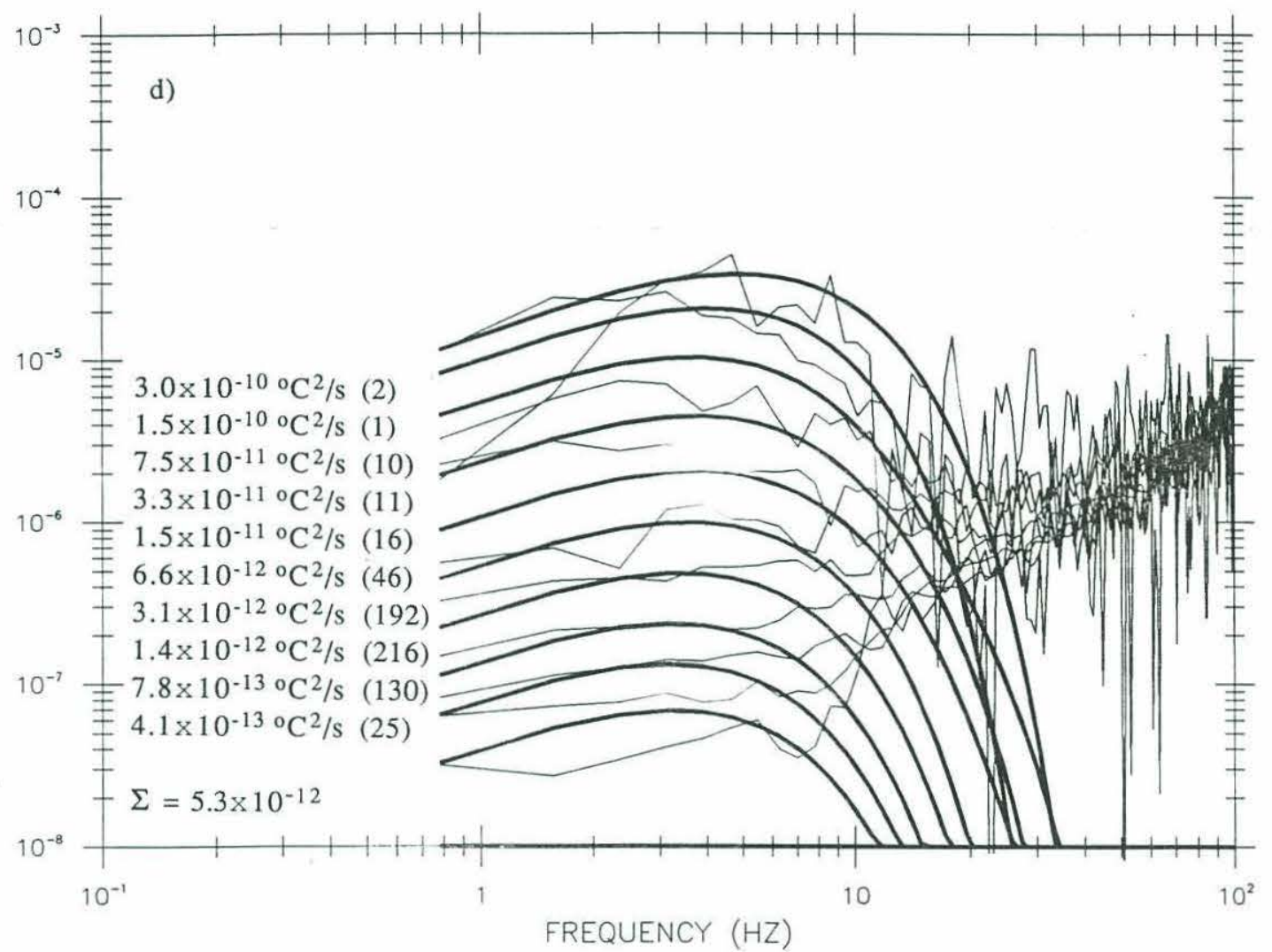

FREQUENCY (HZ)

DIVE 74 2500-3000 TOPO BATCHELOR CZ 
$5 \times 10^{-12}-1 \times 10^{-11}$, etc. and plotted as the thin lines. Overplotted in thick lines are the average of the Nasmyth spectra (Oakey, 1982) for each of the individual spectra. To the left of each spectra are the bin averaged estimates of $\varepsilon$ and the number of spectra in the average. Reasonable (but not exact) agreement is noted for $\varepsilon>1 \times 10^{-11} \mathrm{~W} / \mathrm{kg}$. A similar scheme was applied to the microtemperature (Figure A.1.1 c) and microconductivity (Figure A.1.1 d) gradient spectra and the Batchelor spectra (Oakey, 1982) overplotted. In the case of the microtemperature spectra, reasonable agreement is noted for $\chi>$ $2 \times 10^{-12}{ }^{\circ} C^{2} / s$. Somewhat higher noise levels are noted for the microconductivity spectra. The plots were developed as a diagnostic tool during processing and the dissipation estimates, universal curves and Batchelor spectra were calculated using a constant value of molecular viscosity $\left(v=10^{-6} \mathrm{~m}^{2} / \mathrm{s}\right)$. Molecular values of viscosity and diffusivity based upon Siedler and Peters (1986) were used to create the final values of $\varepsilon$ and $\chi$. Hence the sum of the dissipations in Figures A.1.1 a,b does not equal the quoted value of $4.1 \times 10^{-11}$ $\mathrm{W} / \mathrm{kg}$ and the universal curves are to be viewed as suggestive.

Further confidence can be found in the consistency of the $K_{\rho}$ and $\mathrm{K}_{\mathrm{T}}$ values. Figure A.1.2 is a plot of the two eddy diffusivity estimates versus depth for the TOPO_DEEP reduced data set (Chapter Three). The two estimates agree to within a factor of two. 


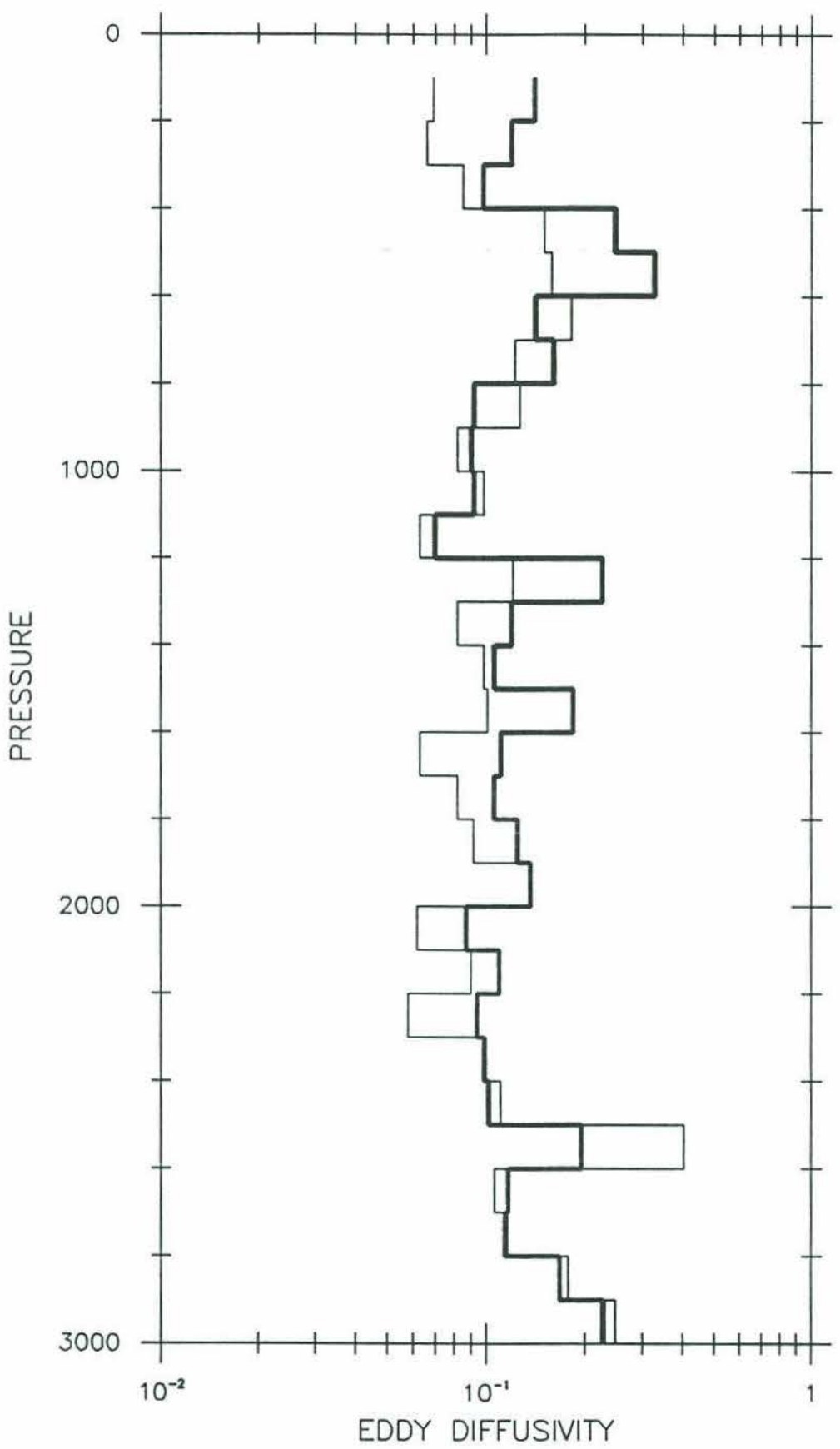

Figure A.1.2: One hundred meter averages of $\mathrm{K}_{\rho}$ and $\mathrm{K}_{\mathrm{T}}$ for the reduced TOPO_DEEP data set. The two estimates agree to within a factor of two. 


\section{Finescale Data}

Estimates of buoyancy frequency and internal wave shear are produced from linear least square fits unless noted. Estimates of buoyancy frequency squared are produced from least square fits to the specific volume anomaly, as in Millard et al. (1990). Estimates of the variable $S^{2}$ are produced from least square fits to the velocity components. Individual estimates of shear are then squared and summed, $S^{2}=\left(u_{z}^{2}+v_{z}^{2}\right)$. A ten meter first difference estimate of shear squared is represented by $\mathrm{S}_{10}^{2}$. It is produced by first differencing the velocities over ten meters, squaring and then summing.

The least squares fit acts as a low pass filter and is occasionally employed to vary the wavenumber content of a variable (e.g. Chapter Four). The effective filter for a $5 \mathrm{~m}$ fit has a $1 / 2$ power point at approximately .1 cpm (Figure A.1.3) and falls off steeply thereafter. The $\mathrm{x}$ meter least squares estimator of slope (i.e. of derivative quantities) has a $1 / 2$ power point at slightly larger wavenumbers than the corresponding ( $x$ meter) first difference operator, and has the advantage of smaller side lobes (Figure A.1.3).

The least squares operator can be used to estimate the spectral level of a variable if the spectral shape for that variable is assumed. If, for instance, a variable is white until a wavenumber $2 \pi / \delta$ and zero thereafter, the least squares fit of length $\delta$ will tend to underestimate the total variance by a factor of 1.86 . The corresponding firstdifference operator will underestimate the total variance by a factor of 2.24. These correction factors are obtained by integrating the 


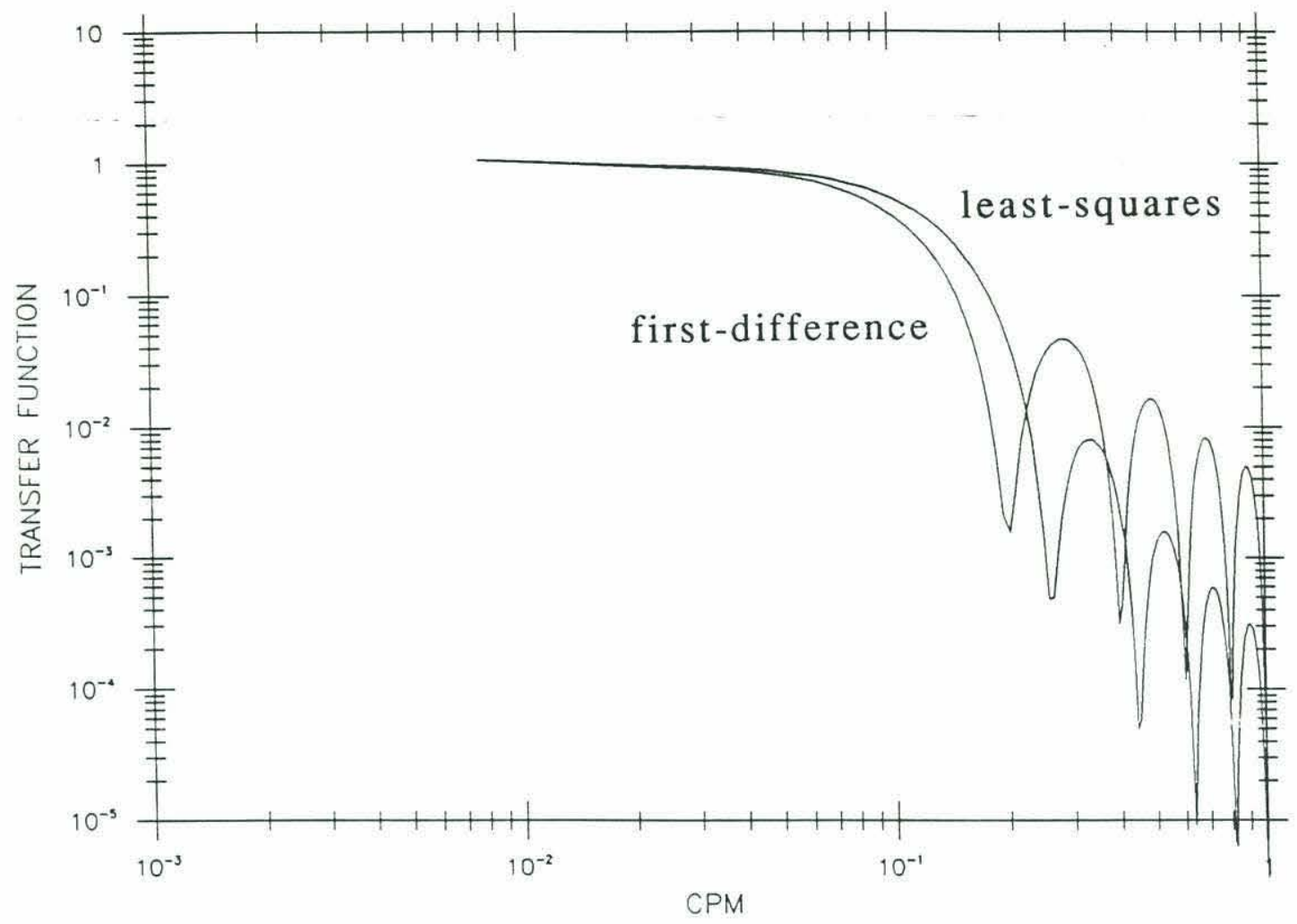

Figure A.1.3: Transfer functions for the five meter least-squares and first-difference operators. 
transfer functions (Figure A.1.3) out to a wavenumber $2 \pi / \delta$ and then dividing the integral of a spectrum which is white until $2 \pi / \delta$ by the result. A similar procedure was employed by Gregg and Sanford (1988).

WKB scaling references a background buoyancy frequency in a quiescent ocean. In this study the background stratification is estimated from the average values of temperature and salinity on a pressure surface from a group of similar stations. Those average values are used to produce least square fit estimates for the background stratification. This procedure is employed to avoid the variability in $\mathrm{N}^{2}$ due to internal wave straining, which can be quite large in highly energetic wave fields. The eight (fifteen) TOPO_DEEP (TOPO_F) profiles were averaged to obtain the background stratification profiles for the respective data sets. For the WRINCLE data, averaged temperature and salinity profiles were created from individual profiles for which the ten degree isotherm was found to be deeper than 425 meters. Thirty-seven of the forty profiles were represented in the average.

The symbol $S_{z}$ (Chapter Three) represents an averaged estimate of the shear spectral density for wavenumbers less than $\mathrm{m}_{\mathrm{c}}$, the cutoff vertical wavenumber, where

$$
\int_{0}^{m_{c}} m^{2} E(m) d m=N^{2} / 2
$$

and $\mathrm{E}(\mathrm{m})$ is the vertical wavenumber energy density spectrum. The estimate $S_{z}$ was produced by: (1) Selecting an appropriate 256(128 $\mathrm{m})$ or $512(256 \mathrm{~m})$ point piece over which to transform. The smaller 
value was generally chosen to limit variability in $\mathrm{N}$ over the selected depth range. For highly energetic regions this did not provide sufficient bandwidth for $\mathrm{m}<\mathrm{m}_{\mathrm{c}}$ to provide reasonably confident limits of $S_{z}$ and thus the larger interval was chosen. (2) The velocity components were then normalized by $N_{0} / N$ where $N_{0}=3 \mathrm{cph}$ and $\mathrm{N}$ represents the average buoyancy frequency over the transform length. (3) The scaled velocity profiles were detrended with a linear fit since wavelengths larger than the interval in question are not well represented by the transform, applied $10 \%$ cosine tapers and prewhitened by first differencing to limit leakage, and then transformed. The resulting Fourier coefficients were 'recolored' to correct for the first difference transfer function and adjusted for the loss of variance due to the tapering operation. Orthogonal components were then squared, summed and multiplied by the wavenumber squared to produce a shear spectrum normalized to 3 cph. (4) These spectra were typically averaged over a large depth bin (e.g. $500 \mathrm{~m}$ ) and over a set of profiles. (5) Finally, $S_{z}$ was produced by integrating (summing the spectral estimates multiplied by (number of points in the transform $x$ decimation interval) ${ }^{-1}$ the averaged spectral estimates until $\mathrm{N}_{0}^{2} / 2$ was exceeded, and then dividing by the bandwidth of the estimate ( the bandwidth being (the number of estimates in the sum) $x$ (number of points in the transform $\mathrm{x}$ decimation interval) ${ }^{-1}$ of the estimate. The endpoint was chosen of the basis of the Munk (1981) spectra:

$$
\int_{0}^{.1 \mathrm{cpm}} \mathrm{m}^{2} \mathrm{E}_{\mathrm{GM}}(\mathrm{m}) \mathrm{dm} \sim \mathrm{N}^{2} / 2 .
$$


The averaged temperature and salinity profiles were also used to create averaged potential density profiles. Internal wave displacements were defined as the difference in pressure of surfaces of constant potential density between the individual and averaged profiles. The profiles of vertical displacement were then spectrally analyzed in the same manner as the velocity profiles to produce strain spectra (strain is defined as the vertical derivative of the vertical displacement). Ideally, the reference density profile should be a time average to limit the possible effects of finestructure contamination. The TOPO_DEEP average potential density profile represented a spatial average and the WRINCLE average potential density profile was a mixed space/time average. 


\section{Appendix Two}

The Garrett and Munk Spectrum

The intent of this appendix is to provide a synopsis of the Garrett and Munk spectrum as presented in Munk (1981) with the intent of elucidating the scaling relationships of Gargett and Holloway (1984) and the consequences of the universal shear spectrum (Gargett, 1990).

Table A.2.1: Notation for the GM spectrum.

$\begin{array}{ll}\mathrm{E}=\mathrm{E}_{\mathrm{GM}} \mathrm{b}^{2} \mathrm{~N}_{\mathrm{o}} \mathrm{N} & \text { total energy } \\ \mathrm{E} & \text { dimensionless spectral level } \\ \mathrm{E}_{\mathrm{GM}}=6.3 \times 10^{-5} & \text { GM specification of } \mathrm{E} \\ \mathrm{b}=1300 \mathrm{~m} & \text { scale height of the thermocline } \\ \mathrm{N} & \text { buoyancy frequency } \\ \mathrm{N}_{\mathrm{o}}=.00524 \mathrm{~s}^{-1} & \text { reference frequency } \\ \mathrm{f}=2 \times 7.3 \times 10^{-5} \sin (\text { lat }) & \begin{array}{l}\text { Coriolis parameter } \\ \mathrm{m}\end{array} \\ \mathrm{m}_{*}=3 \pi \mathrm{N} / \mathrm{bN}_{\mathrm{o}} & \text { vertical wavenumber } \\ \mathrm{k}_{\mathrm{h}} & \text { horizontal wavenumber } \\ \omega & \text { frequency } \\ \mathbf{k} & \text { wavenumber vector } \\ \mathbf{x} & \text { position vector }\end{array}$

The linear, hydrostatic, Boussinesq $\mathrm{w}$ equation is: $\left(\frac{d^{2}}{d t^{2}}+f^{2}\right) \frac{d^{2} w}{d z^{2}}+N^{2}(z)\left(\frac{d^{2} w}{d x^{2}}+\frac{d^{2} w}{d y^{2}}\right)=0$.

For a single wave, the WKB solution for this equation is: $\mathrm{w} \sim \mathrm{N}^{-1 / 2}(\mathrm{z}) \exp \pm \mathrm{i}\left\{\frac{\mathrm{k}_{\mathrm{h}}}{\left(\omega^{2}-\mathrm{f}^{2}\right)^{1 / 2}} \int \mathrm{N}(\mathrm{z}) \mathrm{dz}\right\}$. 
The hydrostatic condition,

$$
\mathrm{N}^{2} \mathrm{w}=-\frac{1}{\rho_{\mathrm{o}}} \frac{\mathrm{d}^{2} \mathrm{p}}{\mathrm{dtd} \mathrm{z}},
$$

implies, for $\mathrm{w} \sim \mathrm{A} \operatorname{exp~iF},(\mathrm{dF} / \mathrm{dz})^{2} \gg(1 / \mathrm{A})\left(\mathrm{d}^{2} \mathrm{~A} / \mathrm{dz}^{2}\right)$, i.e. for wave

scales much less than the scale of change of the background

stratification (the WKB approximation), that $\mathrm{p} \sim \mathrm{N}^{1 / 2}$. From either

horizontal momentum equation, e.g. du/dt $-\mathrm{fv}=-\left(1 / \rho_{\mathrm{o}}\right)(\mathrm{dp} / \mathrm{dx}), \mathrm{u}, \mathrm{v}$

$\sim \mathrm{N}^{1 / 2}$. Since $\mathrm{u}_{\mathrm{z}}=\mathrm{mu}$ and $\mathrm{m} \sim \mathrm{N} ; \mathrm{u}_{\mathrm{z}}, \mathrm{v}_{\mathrm{z}} \sim \mathrm{N}^{3 / 2}$.

These relationships change in a bandwidth limited wavefield.

From Munk (1981):

$$
\begin{aligned}
& F_{\eta}(\omega, j)=b^{2} N_{0} \frac{\left(\omega^{2}-f^{2}\right)}{N \omega^{2}} E(\omega, j) \\
& F_{u}(\omega, j)=b^{2} N_{0} N \frac{\left(\omega^{2}+f^{2}\right)}{\omega^{2}} E(\omega, j)
\end{aligned}
$$

where $\mathrm{E}(\omega, \mathrm{j})=\mathrm{B}(\omega) \mathrm{H}(\mathrm{j}) \mathrm{E}, \mathrm{B}(\omega)=\frac{2 \mathrm{f}}{\pi \omega} \frac{1}{\left(\omega^{2}-\mathrm{f}^{2}\right)^{1 / 2}}$,

$$
\int_{\mathrm{f}}^{\mathrm{N}(\mathrm{z})} \mathrm{B}(\omega) \mathrm{d} \omega=1 \text {, and } \mathrm{H}(\mathrm{j})=\frac{\left(\mathrm{j}^{2}+\mathrm{j}_{*}^{2}\right)^{-1}}{\sum_{1}^{\infty}\left(\mathrm{j}^{2}+\mathrm{j}_{*}^{2}\right)^{-1}}, \sum_{1}^{\infty}\left(\mathrm{j}^{2}+\mathrm{j}_{*}^{2}\right)^{-1}=.468=\mathrm{S}
$$

For $\omega \ll<N, m \sim \frac{\pi N j}{b N_{o}}, \frac{d j}{d m} \sim \frac{b N_{o}}{\pi N}$. Hence,

$$
\begin{aligned}
& F_{\eta}(\omega, m)=\frac{2 b E f}{S} \frac{1}{\left(m^{2}+m_{*}^{2}\right)} \frac{\left(\omega^{2}-f^{2}\right)^{1 / 2}}{w^{3}} \\
& F_{u}(\omega, m)=\frac{2 b N^{2} E f}{S} \frac{1}{\left(m^{2}+m_{*}^{2}\right)} \frac{\left(\omega^{2}+f^{2}\right)}{\omega^{3}\left(\omega^{2}-f^{2}\right)^{1 / 2}}
\end{aligned}
$$


The velocity and displacement variances are obtained by integrating the spectral densities:

$$
\begin{aligned}
& \int_{0}^{\infty} \frac{d m}{\left(m^{2}+m_{*}^{2}\right)}=\frac{\pi}{2 m_{*}}=\frac{b N_{o}}{6 N} \\
& \int_{f}^{N} \frac{\left(\omega^{2}-f^{2}\right)^{1 / 2} d \omega}{\omega^{3}}=\frac{\pi}{4 f} \\
& \int_{f}^{N} \frac{\left(\omega^{2}+f^{2}\right) d \omega}{\omega^{3}\left(\omega^{2}-f^{2}\right)^{1 / 2}}=\frac{3 \pi}{4 f} .
\end{aligned}
$$

Hence $\left\langle\eta^{2}\right\rangle=b^{2} \mathrm{EN}_{\mathrm{o}} / 2 \mathrm{~N}$ and $\left\langle\mathrm{u}^{2}\right\rangle=3 \mathrm{~b}^{2} \mathrm{EN}_{0} \mathrm{~N} / 2$. The buoyancy scalings for the horizontal velocities and the vertical displacements are identical to those predicted by WKB scaling.

Since $w=\frac{d \eta}{d t}, F_{W}(\omega, m)=\frac{2 b E f}{S} \frac{1}{\left(m^{2}+m_{*}^{2}\right)} \frac{\left(\omega^{2}-f^{2}\right)^{1 / 2}}{\omega}$ and

$$
\int_{f}^{N} \frac{\left(\omega^{2}-f^{2}\right)^{1 / 2} d \omega}{\omega}=N
$$

Hence $\left\langle w^{2}\right\rangle=2 b^{2} E_{0} f / 3$, in contrast to the WKB scaling of $N^{-1}$.

Similar changes due to limitations in the vertical bandwidth occur for the derivative quantities of interest, shear and strain:

$$
F_{\eta_{z}}(\omega, m)=\frac{2 b E f}{S} \frac{m^{2}}{\left(m^{2}+m_{*}^{2}\right)} \frac{\left(\omega^{2}-f^{2}\right)^{1 / 2}}{\omega^{3}}
$$




$$
\mathrm{F}_{\mathrm{u}_{\mathrm{z}}}(\omega, \mathrm{m})=\frac{2 \mathrm{bN} N^{2} \mathrm{Ef}}{\mathrm{S}} \frac{\mathrm{m}^{2}}{\left(\mathrm{~m}^{2}+\mathrm{m}_{*}^{2}\right)} \frac{\left(\omega^{2}+\mathrm{f}^{2}\right)}{\omega^{3}\left(\omega^{2}-\mathrm{f}^{2}\right)^{1 / 2}} .
$$

The vertical wavenumber integral is infinite if evaluated at $\mathrm{m}=0$. If one invokes a cut-off wavenumber, $\mathrm{m}_{\mathrm{c}}$, then

$$
\int_{0}^{m_{c}} \frac{m^{2} d m}{\left(m^{2}+m_{*}^{2}\right)}=m_{c} \text {. }
$$

Hence $\left\langle\eta_{\mathrm{z}}^{2}\right\rangle=\pi \mathrm{bEm}_{\mathrm{c}}$ and $\left\langle\mathrm{u}_{\mathrm{z}}^{2}\right\rangle / \mathrm{N}^{2}=3 \pi \mathrm{bEm} \mathrm{m}_{\mathrm{c}}$. Thus vertical wavenumber bandwidth limitations change the buoyancy scaling for the shear and strain variance by a factor of $\mathrm{N}$ from that predicted by WKB theory.

From an observational standpoint there is evidence that $\mathrm{m}_{\mathrm{c}}$ is independent of $\mathrm{N}$ and that the spectra fall off as approximately $\mathrm{m}^{-1}$ for $\mathrm{m}>\mathrm{m}_{\mathrm{c}}$ (Gargett et al., 1981; Gregg et al., 1991; Figure 3.5). There exists additional evidence to suggest that $\mathrm{Em}_{\mathrm{c}}$ is approximately constant (Duda and Cox, 1989; Gregg et al., 1991; Figures 3.5 and 3.12). While the universal shear spectrum described by Gargett (1990) may provide a zeroth order description, both Duda and Cox (1989) and Gregg et al. (1991) note significant exceptions. In Chapter Four a number of dynamically based speculations are forwarded which may potentially explain the observed variability in the shear spectra.

The universal shear spectrum concept has a number of immediate implications for estimates of internal wave stability parameters. First, if $\mathrm{E}$ is intended to represent the spectral level of the low wavenumber $\left(\mathrm{m}<\mathrm{m}_{\mathrm{c}}\right)$ part of the spectrum, any estimate of 
E from $\left\langle\mathrm{u}_{\mathrm{z}}^{2}>/ \mathrm{N}^{2}\right.$ and assuming that the shear spectra is essentially white will produce a biased estimate if the integration is carried out to wavenumbers $m>m_{c}$. This is the basis of Gargett's (1990) criticism of Gregg (1989). Secondly, in as much as shear and strain may be related to instabilities which produce turbulence, the instability parameters are independent of depth and appear to be universal when calculated over a depth interval which varies inversely with $\mathrm{E}$.

Given suitable approximations, the strain field can be simply related to the density gradient. The density field can be written as $\rho$ $=\rho_{0}(z)+\rho^{\prime}(z)$ where $\rho^{\prime}$ is due to internal wave displacements, $\eta$, and $\rho_{0}(z)$ is the time averaged background density profile. Expanding the density perturbations in a Taylor series one obtains (Desaubies and Smith, 1982),

$$
\rho=\rho_{0}(z)+\eta \frac{d \rho_{0}(z)}{d z}+\frac{1}{2} \eta^{2} \frac{d^{2} \rho_{0}(z)}{d z^{2}}+\ldots,
$$

where the expansion is evaluated at equilibrium depth of $\rho_{0}(z)$. Taking the derivative of this equation with respect to $\mathrm{z}$, ignoring $\eta\left(d^{2} \rho_{o}(z) / d z^{2}\right)$ with respect to $(d \eta / d z)\left(d \rho_{0}(z) / d z\right)$, multiplying by ($\left.\mathrm{g} / \rho_{\mathrm{o}}\right)$ and rearranging produces $\left.\left.\eta_{\mathrm{z}}=\left(\mathrm{N}^{2}-<\mathrm{N}^{2}\right\rangle /<\mathrm{N}^{2}\right\rangle\right)$. Two issues are associated with such an expansion. The first is a conceptual one associated with sampling. Desaubies and Gregg (1981) note that since $\eta_{z} \sim O(1)$, the evaluation of the truncated Taylor series expansion needs to be done at the equilibrium depth of a potential density surface rather than the observed pressure of a given isopycnal. Pinkel and Anderson (1992) show that the strain statistics 
are dependant upon whether they are sampled uniformly in pressure or potential density. The second issue is one of finestructure contamination. Truncating the Taylor series implies errors of order $\eta / \mathrm{H}$ where $\mathrm{H}$ is the vertical scale of the background. In most instances the vertical scale of the horizontally averaged background stratification is much larger than the displacement. However, this need not imply that even if the horizontally averaged vertical stratification gradient is constant, that the time averaged vertical gradient is also constant. Mixed space/time averages of the background density profile were calculated in Chapter Three with which to reference the displacement profiles. We are not aware of any studies of the horizontal variability of the time averaged vertical density profile and cannot asses the error which is incurred. 


\section{References}

Abarbanel, H. D. I., D. D. Holm, J. E. Marsden and T. Ratiu, Richardson Number Criterion for the Nonlinear Stability of ThreeDimensional Stratified Flow, Phys. Rev. Letters, 52, 2352-2355, 1984.

Baker, M. A., and C. H. Gibson, Sampling Turbulence in the Stratified Ocean: Statistical Consequences of Strong Intermittency, JPO, 17, 1817-1836, 1987.

Barton, E. D., and A. E. Hill, Abyssal Flow through the Amirante Trench (Western Indian Ocean), DSR, 36, 1121-1126, 1989.

Bell, T. H., Topographically Generated Internal Waves in the Open Ocean, JGR, 80, 320-327, 1975.

Bendat, J. S., and A. G. Piersol, Random Data: Analysis and Measurement Proceedures, Wiley-Interscience, 407 pp., 1971.

Bohrer, P. S., Numerical Simulation of Richardson Number Fluctuations in the Ocean, unpublished manuscript, 1983.

Bradley, J. V., Distribution-Free Statistical Tests, Prentice-Hall, Englewood Cliffs, N. J., 388 pp., 1968.

Briscoe, M. G., Gaussianity of Internal Waves, JGR, 82, 2117-2126, 1977.

Bryan, F., Parameter Sensitivity of Primative Equation Ocean General Circulation Models, JPO, 17, 970-985, 1987.

Cummins, P. F., G. Holloway and A. E. Gargett, Sensitivity of the GFDL Ocean General Circulation Model to a Parameterization of Vertical Diffusion, JPO, 20, 817-830, 1990.

D'Asaro, A. E., Upper Ocean Temperature Structure, Inertial Currents, and Richardson Numbers Observed during Strong Meteorological Forcing, JPO, 15, 943-962, 1985.

D'Asaro, E. A., and H. Perkins, A Near-Inertial Wave Spectrum for the Sargasso Sea in Late Summer, JPO, 14, 489-505, 1984. 
D'Asaro, A. E., and M. D. Morehead, Internal Waves and Velocity

Finestructure in the Artic Ocean, JGR, 96, 12725-12738, 1991.

Desaubies, Y., and M. C. Gregg, Reversible and Irreversible Finestructure, JPO, 11, 541-556, 1981.

Desaubies, Y., and W. K. Smith, Statistics of Richardson Number and Instability in Oceanic Internal Waves, JPO, 12, 1245-1259, 1982.

Dewan, E. M., and R. E. Good, Saturation and the "Universal" Spectrum for Vertical Profiles of Horizontal Scalar Winds in the Atmosphere, JGR, 91, 2742-2748, 1986.

Dillon, T. M., Vertical Overturns: A Comparison of Thorpe and Ozmidov Length Scales, JGR, 87, 9601-9613, 1982.

Duda, T. F., and C. S. Cox, Vertical Wavenumber Spectra of Velocity and Shear at Small Internal Wave Scales, JGR, 94, 939-950, 1989.

Dugan, J. P., and B. W. Stalcup, Comparison of Towed Conductivity Sensor Performance, JOAT, 5, 116-122, 1988.

Dunkerton, T. J., Theory of Internal Gravity Wave Saturation, Pageoph, 130, 373-397, 1989.

Efron, B., and G. Gong, A Leisurely Look at the Bootstrap, the Jackknife, and Cross-Validation, The Am. Stat., 37, 36-48, 1983.

Eriksen, C. C., Observations of Internal Wave Reflection off Sloping Bottoms, JGR, 87, 525-538, 1982.

Eriksen, C. C., Implications of Ocean Bottom Reflection for Internal Wave Spectra and Mixing, JPO, 15, 1145-1156, 1985.

Eriksen, C. C., Variability in the Upper-Ocean Internal Wave Field at a Sargasso Sea Site, JPO, 18, 1495-1513, 1988.

Evans, D. L., Observations of Small-Scale Shear and Density Structure in the Ocean, DSR, 29, 581-595, 1982. 
Frankignoul, C., Observed Anisotropy of Spectral Characteristics of Internal Waves Induced by Low Frequency Currents, JPO, 4, 625-634, 1974.

Frankignoul, C., Observed Interaction between Oceanic Internal Waves and Mesoscale Eddies, DSR, 23, 805-820, 1976.

Frankignoul, C., and T. M. Joyce, On the Internal Wave Variability During the Internal Wave Experiment (IWEX), JGR, 84, 769776, 1979.

Fritts, D. C., A Review of Gravity Wave Saturation Processes, Effects, and Variability in the Middle Atmosphere, Pageoph, 130, 343371, 1989.

Fritts, D. C., and P. K. Rastogi, Convective and Dynamical Instabilities Due to Gravity Wave Motions in the Lower and Middle Atmosphere: Theory and Observations, Radio Sci., 20, 1247$1277,1985$.

Fritts, D. C., and L. Yuan, Stability Analysis of Inertio-Gravity Wave Structure in the Middle Atmosphere, JAS, 46, 1738-1745, 1989.

Fofonoff, N. P., Spectral Characteristics of Internal Waves in the Ocean, DSR, suppl. to 16, 59-71, 1969.

Gargett, A. E., Vertical Eddy Diffusivity in the Ocean Interior, JMR, 42, 359-393, 1984.

Gargett, A. E., Ocean Turbulence, Ann. Rev. Fluid Mech., 419-452, 1989.

Gargett, A. E., Do We Really Know How to Scale the Turbulent Kinetic Energy Dissipation Rate $\varepsilon$ Due to Braking of Oceanic Internal Waves?, JGR, 95, 15971-15974, 1990.

Gargett, A. E., P. J. Hendricks, T. B. Sanford, T. R. Osborn and A. J. Williams III, A Composite Spectrum of Vertical Shear in the Ocean, JPO, 11, 1258-1271, 1981.

Gargett, A. E., and G. Holloway, Dissipation and Diffusion by Internal Wave Breaking, JMR, 42, 15-27, 1984. 
Gargett, A. E., and T. R. Osborn, Small-scale Shear Measurements during the Fine and Microstructure Experiment (FAME), JGR, $86,1929-1944,1981$

Garrett, C., Marginal Mixing Theories, Unpublished manuscript, 1990.

Garrett, C., and W. Munk, Space-Time Scales of Internal Waves, GFD, 2, 225-264, 1972a.

Garrett, C., and W. Munk, Oceanic Mixing by Breaking Internal Waves, DSR, 19, 823-832, 1972 b.

Garrett, C., and W. Munk, Internal Waves in the Ocean, Ann. Rev. Fluid Mech., 11, 339-369, 1979.

Gibson, C. H., Internal Waves, Fossil Turbulence, and Composite Ocean Microstructure Spectra, JFM, 168, 89-117, 1986.

Gonella, J., A Rotary-Component Method for Analyzing Meteorological and Oceanographic Vector Time-Series, DSR, 19, 833-846, 1972.

Gregg, M. C., Microstructure Patches in the Thermocline, JPO, 10, 915943, 1980.

Gregg, M. C., Diapycnal Mixing in the Thermocline: A Review, JGR, 92, 5249-5286, 1987.

Gregg, M. C., Scaling Turbulent Dissipation in the Thermocline, JGR, 94, 9686-9698, 1989.

Gregg, M. C., T. Meagher, A. Pederson and E. Aagaard, Low Noise Temperature Microstructure Measurements with Thermistors, DSR, 25, 843-856, 1978.

Gregg, M. C. and T. Meagher, The Dynamic Response of Glass Rod Thermistors, JGR, 85, 2779-2786, 1980.

Gregg, M. C., E. A. D'Asaro, T. J. Shay and N. Larson, Observations of Persistant Mixing and Near-Inertial Internal Waves, JPO, 16, 856-885, 1986. 
Gregg, M. C., and T. B. Sanford, The Dependence of Turbulent Dissipation on Stratification in a Diffusively Stable Thermocline, JGR, 93, 12,381-12,392, 1988.

Gregg, M. C., and E. Kunze, Internal Wave Shear and Strain in Santa Monica Basin, JGR, 96, 16709-16719, 1991.

Gregg, M. C., D. P. Winkel and T. B. Sanford, Internal Wave Shear and Dissipation, Dynamics of Oceanic Internal Gravity Waves, Proceedings 'Aha Huliko'a Hawaiian Winter Workshop, P. Muller and D. Henderson, Eds., 1-30, 1991.

Hanson, H. P., P. Cornillon, G. R. Halliwell, Jr. and V. Halliwell, Climatological Perspectives, Oceanogrphic and Meteorological, on Variability in the Subtropical Convergence Zone in the Northwestern Atlantic, JGR, 96, 8517-8529, 1991.

Hayes, S. P., H. B. Milburn, and E. F. Ford, TOPS: A Free-Fall Velocity and CTD Profiler, JOAT, 1, 220-236, 1984.

Hazel, P., Numerical Studies of the Stability of Inviscid Stratified Shear Flows, JFM, 51, 39-61, 1972.

Head, M. J., The use of Miniature Four-Electrode Conductivity Probes for High Resolution Measurement of Turbulent Density or Temperature Variations in Salt-Stratified Water Flows, Ph.D. dissertation, University of California at San Diego, 211 pp, 1983.

Henyey, F. S., Hamiltonian Description of Startified Fluid Dynamics, Phys. Fluids, 26, 40-47, 1983.

Henyey, F., Transport of Small-Scale Internal Waves Toward Microstructure, Internal Gravity Waves and Small-Scale Turbulence, Proceedings 'Aha Huliko'a Hawaiian Winter Workshop, P. Muller and R. Pujalet, Eds., 201-220, 1984.

Henyey, F. S., Scaling of Internal Wave Model Predictions for $\varepsilon$, Dynamics of Oceanic Internal Gravity Waves, Proceedings 'Aha Huliko'a Hawaiian Winter Workshop, P. Muller and D. Henderson, Eds., 233-236, 1991. 
Henyey, F. S. and N. Pomphrey, Eikonal Description of Internal Wave Interactions: A Non-Diffusive Picture of "Induced Diffusion", DAO, 7, 189-219, 1983.

Henyey, F. S., J. Wright and S. M. Flatte', Energy and Action Flow Through the Internal Wave Field: an Eikonal Approach, JGR, 91, 8487-8495, 1986.

Hines, C. O., Generation of Turbulence by Atmospheric Gravity Waves, JAS, 45, 1269-1378, 1988.

Hines, C. O., The Saturation of Gravity Waves in the Middle Atmosphere. Part I: Critique of Linear-Instability Theory, JAS, 48, 1348-1359, 1991a.

Hines, C. O., The Saturation of Gravity Waves in the Middle Atmosphere. Part II: Development of Doppler-Spread Theory, JAS, 48, 1360-1379, $1991 \mathrm{~b}$.

Hines, C. O., The Saturation of Gravity Waves in the Middle Atmosphere. Part III: Formation of the Turbopause and of Turbulent Layers beneath It, JAS, 48, 1380-1385, 1991c.

Hines, C. O., The Saturation of Middle-Atmosphere Gravity Waves, Dynamics of Oceanic Internal Gravity Waves, Proceedings 'Aha Huliko'a Hawaiian Winter Workshop, P. Muller and D. Henderson, Eds., 261-278, 1991d.

Hogg, N. G., P. Biscaye, W. Gardner, and W. J. Schmitz, On the Transport and Modification of Antarctic Bottom Water in the Vema Channel, JMR, 40, 231-263, 1982.

Holloway, G., On Interaction Time Scales of Oceanic Internal Waves, JPO, 12, 293-296, 1982.

Holloway, G., A conjecture Relating Oceanic Internal Waves and Small-Scale Processes, Atmos. Ocean, 21, 107-122, 1983.

Howard, L. N., Note on a Paper of John W. Miles, JFM, 10, 509-512, 1961.

Itsweire, E. C., Measurements of Vertical Overturns in a Stably Stratified Turbulent Flow, Phys. of Fluids, 27, 764-766, 1984. 
Itsweire, E. C., K. N. Helland, C. W. Van Atta, The Evolution of GridGenerated Turbulence in a Stably Stratified Fluid, JFM, 162, 299-338, 1986.

Jenkins, W. J., Tritium and ${ }^{3} \mathrm{He}$ in the Sargasso Sea, JMR, 38, 533-569, 1980.

Johnson, G. C., Near-Equatorial Deep Circulation in the Indian and Pacific Oceans, Ph. d. Thesis, MIT/WHOI, pp. 157, 1990.

Joyce, T. M., B. A. Warren and L. D. Talley, The Geothermal Heating of the Abyssal Subarctic Pacific Ocean, DSR, 33, 1003-1015, 1986.

Kunze, E., Near-Inertial Wave Propagation in Geostrophic Shear, JPO, 15, 544-565, 1985.

Kunze, E., The Mean and Near-Inertial Velocity Fields in a Warm-Core Ring, JPO, 16, 1444-1461, 1986.

Kunze, E., and T. B. Sanford, Observations of Near-Inertial Waves in a Front, JPO, 14, 566-581, 1984.

Kunze, E., A. J. Williams, III, and M. G. Briscoe, Observations of Shear and Vertical Stability from a Neutrally Buoyant Float, JGR, 95, 18127-18142, 1990.

Kunze, E., and T. B. Sanford, Measurements of Ertel Vorticity Finestructure in the Eastern North Atlantic, Dynamics of Oceanic Internal Gravity Waves, Proceedings 'Aha Huliko'a Hawaiian Winter Workshop, P. Muller and D. Henderson, Eds., 157-177, 1991.

Kunze, E., M. A. Kennelly and T. B. Sanford, The Depth Dependence of Shear Finestructure off Point Arena and near Pioneer Seamount, JPO, 22, 29-41, 1992.

Leaman, K. D., Observations of the Vertical Polarization and Energy Flux of Near-Inertial Waves, JPO, 6, 894-908, 1976.

Leaman, K. D., and T. B. Sanford, Vertical Energy propagation of Inertial Waves: A Vector Spectral Analysis of Velocity Profiles, JGR, 80, 1975-1978, 1975. 
Lueck, R. G., O. Hertzman and T. R. Osborn, The Spectral Response of Thermistors, DSR, 24, 951-970, 1977.

Lueck, R. G. and T. R. Osborn, The Characteristics of Internally Heated Thermistors, DSR, 27, 273-292, 1980.

Lueck, R. G., W. R. Crawford and T. R. Osborn, Turbulent Dissipation over the Continental Slope off Vancouver Island, JPO, 13, 1809$1818,1983$.

Lueck, R. G., and T. Osborn, The Dissipation of Kinetic Energy in a Warm-Core Ring, JGR, 91, 803-818, 1986.

Marmarino, G. O., J. P. Dugan and T. E. Evans, Horizontal Variability of Microstructure in the Vicinity of a Sargasso Sea Front, JPO, 16, 967-980, 1986.

Marmarino, G. O., L. J. Rosenblum and C. L. Trump, Fine-scale Temperature Variability: The Influence of Near-Inertial Internal Waves, JGR, 92, 13049-13062, 1987.

McComas, C. H., and P. Muller, The Dynamic Balance of Internal Waves, JPO, 11, 970-986, 1981.

McDougall, T. J., Dianeutral Advection, in Parameterization of SmallScale Processes, Proceedings 'Aha Huliko'a Hawaiian Winter Workshop, University of Hawaii at Manoa, P. Muller and D. Henderson, Ed.s, 289-316, 1989.

McIntyre, M. E., Diffusive Destabilization of the Baroclinic Circular Vortex, GFD, 1, 19-57, 1970.

Meagher, T., A. Pedersen and M. Gregg, A Low Noise Conductivity Microstructure Instrument, Proc. of IEEE Oceans '82, 282-290.

Mied, R. P., C. Y. Shen, C. L. Trump and G. J. Lindemann, InertialInternal Waves in a Sargasso Sea Front, JPO, 16, 1751-1762, 1986.

Mied, R. P., G. J. Lindemann and C. L. Trump, Inertial Wave Dynamics in the North Atlantic Subtropical Zone, JGR, 92, 13063-13074, 1987. 
Mied, R. P., C. Y. Shen and M. Kidd, Current Shear - Inertial Wave Interaction in the Sargasso Sea, JPO, 20, 81-96, 1990.

Miles, J. W., On the Stability of Heterogeneous Shear Flows, JFM, 10, 496-508, 1961.

Millard, R. C., W. B. Owens and N. P. Fofonoff, On the Calculation of the Brunt-Vaisala Frequency, DSR, 37, 167-181, 1990.

Mooers, C. N. K., A Technique for the Cross Spectrum Analysis of Pairs of Complex-Valued Time Series, with Emphasis on the Properties of Polarized Components and Rotational Invariants, DSR, 20, 1129-1141, 1973.

Moum, J. N. and T. R. Osborn, Mixing in the Main Thermocline, JPO, 16, 1250-1259, 1986.

Muller, P., G. Holloway, F. Henyey and N. Pomphrey, Nonlinear Interactions Among Internal Gravity Waves, R. of Geophys., 24,493-536, 1986.

Muller, P., On the Diffusion of Momentum and Mass by Internal Gravity Waves, JFM, 77, 789-823, 1976.

Munk, W., Abyssal Recipes, DSR, 13, 707-730, 1966.

Munk, W., Internal Waves and Small-Scale Processes, In Evolution of Physical Oceanography, B. A. Warren and C. Wunsch, Eds., The MIT Press, Cambridge, 264-291, 1981.

Ninnis, R., The Effects of Spatial Averaging on Airfoil Probe Measurements of Oceanic Velocity Microstructure, Ph.D. dissertation, 109 pp., University of British Columbia, Vancouver, 1984.

Oakey, N. S., An Instrument to Measure Oceanic Turbulence and Microstructure, Bedford Inst. Oceanogr. Rep. Ser. BI-R-77-3, 52 pp, 1977.

Oakey, N. S., Determination of the Rate of Dissipation of Turbulent Energy From Simultaneous Temperature and Velocity Microstructure Measurements, JPO, 12, 256-271, 1982. 
Oakey, N. S., EPSONDE: An Instrument to Measure Turbulence in the Deep Ocean, IEEE Jour. of O.E., 13, 124-128, 1988.

Okawa, B. S., and J. P. Dugan, Contamination of Conductivity Measurements by Waterborn Particles, Ocean Eng., 11, 265279, 1984.

Olbers, D., Diffusion Parameterizations for the Climatological Circulation of the North Atlantic and Southern Ocean, in Parameterization of Small-Scale Processes, Proceedings 'Aha Huliko'a Hawaiian Winter Workshop, University of Hawaii at Manoa, P. Muller and D. Henderson, Ed.s, 181-203, 1989.

Orlanski, I., and K. Bryan, Formation of the Thermocline Step Stucture by Large Amplitude Internal Gravity Waves, JGR, 74, 69756983, 1969.

Osborn, T. R., Vertical Profiling of Velocity Microstructure, JPO, 4,109115, 1974.

Osborn, T. R., Estimates of the Local Rate of Vertical Diffusion from Dissipation Measurements, JPO, 10, 83-89, 1980.

Osborn, T. R, and C. S. Cox, Oceanic Fine Structure, GFD, 3, 321-345, 1972.

Osborn, T. R., and T. E. Siddon, Oceanic Shear Measurements Using the Oceanic Shear Probe, Proc. 3rd Biennial Symposium on Turbulence in Liquids, G. K. Patterson and J. L. Zaken, Eds., University of Missouri-Rolla, 1975.

Osborn, T. R., and W. R. Crawford, Turbulent Velocity Measurements with an Airfoil Shear Probe, Manuscript Report $\underline{31}$, University of British Columbia, Vancouver, B.C., 1977.

Osborn, T. R. and W. R. Crawford, An Airfoil probe for Measuring Turbulent Velocity Fluctuations in Water, Air-Sea Interactions: Instruments and Methods, F. Dobson, L. Hasse and R. Davis, Eds., Plenum Press, 801 pp, 1980. 
Perrson, J., Variability and Covariability of Modified Spectral Estimators, IEEE Tans. Acous., Speech Signal Process., 158-160, April, 1974.

Pickart, R. S., N. G. Hogg and W. M. Smethie, Determining the Strength of the Deep Western Boundary Current Using the Chlorofluoromethane Ratio, JPO, 19, 940-951, 1989.

Pinkel, R., J. Sherman, J. Smith and S. Anderson, Strain: Observations of the Vertical Gradient of Isopycnal Vertical Displacement, JPO, 21, 527-540, 1991.

Pinkel, R., and S. Anderson, Toward a Statistical Description of Finescale Strain in the Thermocline, JPO, 22, 773-795, 1992.

Pollard, R. T., Frontal surveys with a Towed Profiling Conductivity/ Temperature/Depth Measurement Package (SeaSoar), Nature, 323, 433-435, 1986.

Pollard, R. T., and L. A. Regier, Vorticity and Vertical Circulation at an Ocean Front, JPO, 22, 609-625,1992.

Rhines, P., and P. MacCready, Boundary Control over the Large-Scale Circulation, in Parameterization of Small-Scale Processes, Proceedings 'Aha Huliko'a Hawaiian Winter Workshop, University of Hawaii at Manoa, P. Muller and D. Henderson, Ed.s, 75-98, 1989.

Rice, S. O., The Mathematical Analysis of Random Noise, Bell System Tech. J., 23, 282-332, 1984, reprinted in Selected Papers on Noise and Stochastic Processes, N. Wax, editor, Dover, 1954.

Rohr, J. J., E. C. Itsweire and C. W. Van Atta, Mixing Efficiency in Stably-Stratified Decaying Turbulence, GAFD, 29, 221-236, 1984.

Rohr, J. J., E. C. Itsweire, K. N. Helland, and C. W. Van Atta, An Investigation of the Growth of Turbulence in a Uniform- MeanShear Flow, JFM, 187, 1-33, 1988.

Rubenstein, D. M., and G. O. Roberts, Scattering of Inertial Waves by an Ocean Front, JPO, 16, 121-131, 1986. 
Ruddick, B. R., Observations of Interaction Between the Internal Wave Field and Low Frequency Flows in the North Atlantic, Ph. D. Thesis, WHOI/MIT, pp 330, 1977.

Ruddick, B. R., Critical Layers and the Garrett-Munk Spectrum, JMR, $38,135-145,1980$.

Ruddick, B. R., and T. M. Joyce, Observations of Interaction between the Internal Wavefield and Low-Frequency Flows in the North Atlantic, JPO, 9, 498-517, 1979.

Saunders, P. M., Flow through Discovery Gap, JPO, 17, 631-643, 1987.

Schmitt, R. W., J. M. Toole, R. L. Koehler, E. C. Mellinger, and K. W. Doherty, The Development of a Fine- and Microstructure Profiler, JOAT, 5, 484-500, 1988.

Scotti, R. S., and G. M. Corcos, An Experiment on the Stability of Small Disturbances in a Stratified Free Shear Layer, JFM, 52, 1972.

Siedler, G. and H. Peters, Physical Properties (General) of Sea Water, in Numerical Data and Functional Relationships in Science and Technology, Landolt-BornsteinJ. Sundermann, Ed., 233-264, 1986.

Smith, S. A., D. C. Fritts and T. E. VanZandt, Evidence for a Saturated Spectrum of Atmospheric Gravity Waves, JAS, 44, 1404-1410, 1987.

Stommel, H., and A. B. Arons, On the Abyssal Circulation of the World Ocean. I. Stationary Planetary Flow Patterns on a Sphere, DSR, $6,140-154,1960$.

Tennekes, H., and J. L. Lumley, A First Course in Turbulence, The MIT Press, Cambridge, pp. 300, 1972.

Thorpe, S. A., Experiments on the Instability of Statified Shear Flows: Miscible Flows, JFM, 46, 299-319, 1971.

Thorpe, S. A., Turbulence in Stably Statified Fluids: A Review of Laboratory Experiments, BLM, 5, 95-119, 1973a. 
Thorpe, S. A., Experiments on Instability and Turbulence in a Stratified Shear Flow, JFM, 61, 731-751, 1973b.

Thorpe, S. A., Transitional Phenomena and the Development of Turbulence in Stratified Fluids: A Review, JGR, 92, 5231-5248, 1987.

Toole, J. M., and S. P. Hayes, Finescale Velocity-Density Characteristics and Richardson Number Statistics of the Eastern Equatorial Pacific, JPO, 14, 712-726, 1984.

Toole, J. M., and R. W. Schmitt, Small-Scale Structures in the NorthWest Atlantic Sub-Tropical Front, Nature, 327, 47-49, 1987.

Wang, D., Generation and Propagation of Inertial Waves in the Subtropical Front, JMR, 619-633, 1991.

Weller, R. A., The Relation of Near-Inertial Motions Observed in the Mixed Layer During JASIN (1978) Experiment to the Local Wind Stress and to the Quasi-Geostrophic Flow Field, JPO, 12, 1122-1136, 1982.

Weller, R. A., Near-Surface Velocity Variability at Inertial and Subinertial Frequencies in the Vicinity of the California Current, JPO, 15, 372-385, 1985.

Weller, R. A., D. L. Rudnick, C. C. Eriksen, K. L. Polzin, N. S. Oakey, J. W. Toole, R. W. Schmitt and R. T. Pollard, Forced Ocean Response During the Frontal Air-Sea Interaction Experiment (FASINEX), JGR, 96, 8611-8638, 1991.

Whitehead, J. A., Surges of Antarctic Bottom Water into the North Atlantic, JPO, 19, 853-861, 1989.

Willebrand, J., P. Muller and D. Olbers, Inverse Analysis of the Trimoored Internal Wave Experiment (IWEX), Ber. Inst. Meeres., Kiel, 20a, b, 117 pp., 1977.

Wunsch, C., and S. Webb, The Climatology of Deep Ocean Internal Waves, JPO, 235-243, 1979.

Wyrtki, K, The Thermohaline Circulation in Relation to the General Circulation in the Oceans, DSR, 39-64, 1961. 
Yamazaki, H., Statified Turbulence Near a Critical Dissipation Rate, JPO, 20, 1583-1594, 1990.

Yamazaki, H. and T. R. Osborn, Dissipation Estimates for Stratified Turbulence, JGR, 95, 9739-9744, 1990. 